NBSIR 83-1685

\title{
GAS ORIFICE METER DISCHARGE COEFFICIENTS AS DETERMINED BY MASS FLOW MEASUREMENTS
}

D.B. Mann

J.A. Brennan

C.F. Sindt

J.F. LaBrecque

S.E. McManus

C.H. Kneebone

National Bureau of Standards

U.S. Department of Commerce

Boulder, Colorado 80303

August 1983

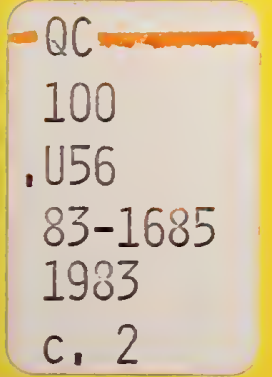



NBSIR 83-1685

\section{GAS ORIFICE METER DISCHARGE COEFFICIENTS AS DETERMINED BY MASS FLOW MEASUREMENTS}

D.B. Mann

J.A. Brennan

C.F. Sindt

J.F. LaBrecque

S.E. McManus

C.H. Kneebone

Chemical Engineering Science Division

National Engineering Laboratory

National Bureau of Standards

U.S. Department of Commerce

Boulder, Colorado 80303

August 1983

Sponsored by:

Gas Research Institute

8600 West Bryn Mawr Avenue

Chicago, Illinois 60631

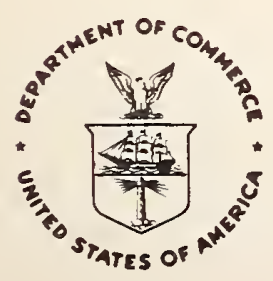

U.S. DEPARTMENT OF COMMERCE, Malcolm Baldrige, Secretary 

1. INTRODUCTION $\ldots \ldots \ldots \ldots \ldots \ldots \ldots \ldots \ldots \ldots \ldots \ldots \ldots \ldots$

2. REFERENCE FLOW SYSTEM $\ldots \ldots \ldots \ldots \ldots \ldots \ldots \ldots \ldots \ldots \ldots$

2.1 Components........................... 2

2.2 Instrumentation...................... 4

2.2.1 Instruments for Measurement

of Mass Flow. .................... 4

2.2.2 Instrumentation on the Gas

Portion of the system............... 4

2.3 Automatic Data Acquisition.................. 5

2.4 Operation........................... 6

3. ORIFICE METER GAS FLOW TESTS................. 7

3.1 Orifice Flowmeter Test Equi pment............ 8

3.1.1 Orifice Meter Runs................ 8

3.1 .2 Orifice Plates................... 8

3.1.3 Flow Conditioners................. 10

3.1.4 Gas System Piping.................. 10

3.2 Orifice Meter Instrumentation.............. 13

3.3 Flow Test Procedures..................... 15

3.4 Test Results......................... 17

3.5 Future Plans......................... 17

REFERENCES CITED......................... 94

APPENDIX I. Measurements of Orifice Meter Runs..... 95

APPENDIX II. Measurements of Orifice Plates ......... 104

APPENDIX III. Measurements of Flow Conditioners........ 121 
Table 1. Orifice Plate-Meter Run Test Combinations

Table 2(a) Test Meter Identification and Physical Measurements Nominal 2 Inch AGA/API Orifice Meter (SI Units)

Table 2(b) Test Number, Measured and Calculated Quantities Nominal 2 Inch AGA/API Orifice Meter (SI Units)

Table 3(a) Test Meter Identification and Physical Measurements Nominal 3 Inch AGA/API Orifice Meter (SI Units)

Table 3(b) Test Number, Measured and Calculated Quantities Nominal 3 Inch AGA/API Orifice Meter (SI Units)

Table 4(a) Test Meter Identification and Physical Measurements Nominal 4 Inch AGA/API Orifice Meter (SI Units)

Table 4(b) Test Number, Measured and Calculated Quantities Nominal 4 Inch AGA/API Orifice Meter (SI Units)

Table 5(a) Test Meter Identification and Physical Measurements Nominal 6 Inch AGA/API Orifice Meter (SI Units)

Table 5(b) Test Number, Measured and Calculated Quantities Nominal 6 Inch AGA/API Orifice Meter (SI Units)

Table 6(a) Test Meter Identification and Physical Measurements Nominal 2 Inch AGA/API Orifice Meter (British Units)

Table 6(b) Test Number, Measured and Calculated Quantities Nominal 2 Inch AGA/API Orifice Meter (British Units)

Table 7(a) Test Meter Identification and Physical Measurements Nominal 3 Inch AGA/API Orifice Meter (British Units)

Table 7 (b) Test Number, Measured and Calculated Quantities Nominal 3 Inch AGA/API Orifice Meter (British Units) 
Table $8(a)$ Test Meter Identification and Physical Measurements Nominal 4 Inch AGA/API Orifice Meter (British Units)

Table $8(b)$ Test Number, Measured and Calculated Quantities Nominal 4 Inch AGA/API Orifice Meter (British Units)

Table 9(a) Test Meter Identification and Physical Measurements Nominal 6 Inch AGA/API Orifice Meter (British Units)

Table 9(b) Test Number, Measured and Calculated Quantities Nominal 6 Inch AGA/API Orifice Meter (British Units) 
Figure 1 Flow reference facility schematic

Figure 2 Typical orifice meter flange

Figure. 3 Flow conditioner

Figure 4 Gas system piping schematic

Figure 5(a) Results from 2 inch meter with the $1-1 / 8$ inch orifice plate

Figure $5(\mathrm{~b})$ Results from 2 inch meter with the $1-3 / 8$ inch orifice plate

Figure $5(\mathrm{c})$ Results from 2 inch meter with the $1-1 / 2$ inch orifice plate

Figure 6(a) Results from 3 inch meter with the 1-1/8 inch orifice plate

Figure 6(b) Results from 3 inch meter with the $1-1 / 2$ inch orifice plate

Figure $6(\mathrm{c})$ Results from 3 inch meter with the $1-3 / 4$ inch orifice plate

Figure 6(d) Results from 3 inch meter with the 2 inch orifice plate

Figure $6(\mathrm{e})$ Results from 3 inch meter with the $2-1 / 4$ inch orifice plate

Figure $7(a)$ Results from 4 inch meter with the 1-1/2 inch orifice plate

Figure $7(b)$ Results from 4 inch meter with the 2 inch orifice plate

Figure $7(\mathrm{c})$ Results from 4 inch meter with the $2-1 / 4$ orifice plate

Figure $7(d)$ Results from 4 inch meter with the 2-5/8 inch orifice plate

Figure $7(\mathrm{e})$ Results from 4 inch meter with the 3 inch orifice plate

Figure $8(a)$ Results from 6 inch meter with the $1-1 / 4$ inch orifice plate

Figure 8 (b) Results from 6 inch meter with the 2-1/4 inch orifice plate

Figure $8(\mathrm{c})$ Results from 6 inch meter with the 3 inch orifice plate

Figure $8(d)$ Results from 6 inch meter with the $3-1 / 2$ inch orifice plate

Figure 9 Plot showing Reynolds number ranges for original Ohio State water data [13] and data from this work 
UNITS AND DIMENSIONS

International System (SI) and U.S. Customary (British)

Unit Names and Symbols

$\begin{array}{llccc}\text { Quantity } & \begin{array}{c}\text { SI Unit } \\ \text { Name }\end{array} & \begin{array}{c}\text { SI Unit } \\ \text { Symbol }\end{array} & \begin{array}{c}\text { British Unit } \\ \text { Name }\end{array} & \begin{array}{c}\text { British Unit } \\ \text { Symbol }\end{array} \\ \text { length } & \text { meter } & \mathrm{m} & & \\ \text { mass } & \text { kilogram } & \mathrm{kg} & \text { foot } & \mathrm{ft} \\ \text { time } & \text { second } & \mathrm{s} & \text { pound } & 1 \mathrm{~b} \\ \text { temperature } & \text { kelvin } & \mathrm{K} & \text { second } & \mathrm{s} \\ & & & \text { fahrenheit } & { }^{\circ} \mathrm{F}\end{array}$

Conversion Factors for Tabular Listings

$\begin{array}{llc}\text { to convert } & \text { to } & \text { multiply by } \\ \mathrm{ft} & \mathrm{m} & 0.30480 \\ { }^{\circ} \mathrm{F} & \mathrm{K} & \mathrm{T}_{\mathrm{K}}=\left(\mathrm{t}_{\mathrm{F}} \mathrm{F}+459.67\right) / 1.8 \\ \mathrm{psia} & \mathrm{MPa} & 6.8948\left(10^{-3}\right) \\ \text { in } \mathrm{H}_{2} \mathrm{O}\left(60^{\circ} \mathrm{F}\right) & \mathrm{kPa} & 0.248843 \\ 1 \mathrm{~b}_{\mathrm{m}} / \mathrm{ft}^{3} & \mathrm{~kg} / \mathrm{m}^{3} & 16.01846\end{array}$

Note: In addition to the tabular listings, we occasionally use U.S. Customary Units (non-SI) within the text to accommodate the intended audience. 

GAS ORIFICE METER DISCHARGE COEFFICIENTS AS DETERMINED BY

MASS FLOW MEASUREMENTS

\author{
D. B. Mann, J. A. Brennan, C. F. Sindt, \\ J. F. LaBrecque, S. E. McManus and C. H. Kneebone \\ National Bureau of Standards \\ Boulder, CO 80303
}

\begin{abstract}
Performance data of gas orifice meter runs and plates have been generated under a U.S. gas industry supported program. The data have been developed using nitrogen gas and a unique NBS gas flow measurement facility capable of directly measuring the mass of gas metered by the orifice device. Direct comparison of predictions from empirical equations can now be made at orifice bore Reynolds Numbers near four million. Two meter runs for each of four nominal line sizes and two sets of orifice plates with up to six beta ratios per set were interchanged in order to develop full meter performance characteristics. Orifice meter and flow reference system data are used to calculate discharge and expansion coefficients which in turn are compared to those derived from existing equations. Orifice meter performance data and system descriptions are provided.
\end{abstract}

Key words: discharge coefficients; flow measurement; flowmeter; gas flow; mass flow; orifice meter.

\title{
1. INTRODUCTION
}

Staff members of the National Bureau of Standards (NBS) laboratories located at Boulder, Colorado have modified an existing cryogenic liquid flowmetering facility to allow precise flow measurement of gas at ambient temperatures. The gas meter measurement is compared directly to the existing liquid mass flow reference system which is based on mass and time measurements [1]. An extensive gas flowmetering research program based on this new facility is in progress. The program was initially supported by the American Gas Association (AGA) and the Gas Research Institute (GRI) and is currently funded entirely by the Gas Research Institute (Contract 5080-353-0422). The cryogenic liquid measurement part of the program continues under sponsorship of the Compressed Gas Association (CGA).

The concept of extending the cryogenic flow reference system to gas flow operation was proposed by Mann and Brennan [2] in 1977. Funding to modify the existing facility to demonstrate the feasibility of gas flow operation was made available from NBS and modifications were completed in July of 1979. The gas flowmetering research program is directed through the GRI-NBS Basic Gas Metering Steering Committee which plans program objectives, reviews current progress and aids NBS in the selection of experiments directed at solutions to current gas metering problems.

The research area of immediate interest to the Committee was the performance of the gas orifice meter. A gas turbine meter was included in the program and was used as a facility monitor for setting flow rates. Future research is planned on the performance of gas turbine flowmeters and the devices.

The Committee provided two sets of orifice meter runs in four nominal line sizes with orifice plates which provided six beta ratios ranging from 0.2 to 
0.75 for each run size. Three flow conditioners were also provided by the Committee. The orifice meter runs, plates, and flow conditioners were fabricated to natural gas industry (U.S.) standards and are the joint property of the American Gas Association and the American Petroleum Institute (AGA/API). Using these multiple sets of runs and plates, a more representative range of meter performance was possible compared to that of a single run size and one or two special orifice plates. A description of the flow reference system, the orifice meters under test and the test procedures are given here in detail. Data are presented in both tabular and graphical form. Archival data are also available [3] and a summary report of this program appears in the literature [4].

Data presented provide added confirmation that suspected gas orifice metering performance anomalies exist in the flow range covered. The data do not necessarily reveal sources of these anomalies at this time and research is continuing in order to establish a rational basis for what needs to be done to significantly improve or replace present correlations.

\section{REFERENCE FLOW SYSTEM}

\subsection{Components}

The flow reference system is shown schematically in figure 1 and consists of a closed loop thermodynamic cycle where the process fluid, nitrogen, is circulated between temperature limits of $85 \mathrm{~K}$ and $300 \mathrm{~K}$ at pressures of $0.5 \mathrm{MPa}$ to $4.1 \mathrm{MPa}$. Work is done on the cycle by centrifugal pumps operating at $85 \mathrm{~K}$ which increase the liquid nitrogen pressure from $0.5 \mathrm{MPa}$ to $4.1 \mathrm{MPa}$. Heat energy enters the process from the ambient surroundings and through a steam heat exchanger which controls the gas temperature at the test section. Heat energy is removed from the system by the refrigeration effect provided by boiling liquid nitrogen in the subcooler, supplementary liquid nitrogen introduced at the main heat exchanger and water cooling at the water heat exchanger following the gas test section.

The low pressure cryogenic portion of the cycle is maintained at a pressure of $0.5 \mathrm{MPa}$ by introducing helium gas at the catch and weigh tank. This inert pressurant provides necessary over-pressure to inhibit boiling of the liquid nitrogen throughout the cryogenic portion of the cycle. The high pressure portion of the cycle is accomplished in two steps. The variable speed centrifugal boost pump provides a controlled pressure of about $0.7 \mathrm{MPa}$ at the inlet to a fixed speed pressure pump. The centrifugal pressure pump then increases the pressure of the process fluid to $4.1 \mathrm{MPa}$. Process fluid flow is controlled by operation of the expansion valve. The pressure level of $4.1 \mathrm{MPa}$ was selected because the critical pressure of nitrogen is $3.4 \mathrm{MPa}$ and operation above this pressure avoids two phase boiling and condensing in the main heat exchanger; a possible source of flow noise.

The main heat exchanger is a five pass, plate-fin type constructed of aluminum and insulated with a $30 \mathrm{~cm}$ thickness of polyurethane foam. The cross section of the heat exchanger is $63.5 \mathrm{~cm}$ by $33 \mathrm{~cm}$ and is 4.92 meters in overall length including manifolds. Two of the five passes provide counter current heating and cooling of the process fluid. Two additional passes contain liquid nitrogen supplemental refrigeration. The final pass is blocked at both ends and is used as a gas thermometer for indication and control of heat exchanger pressures and temperatures during the test draft period. 


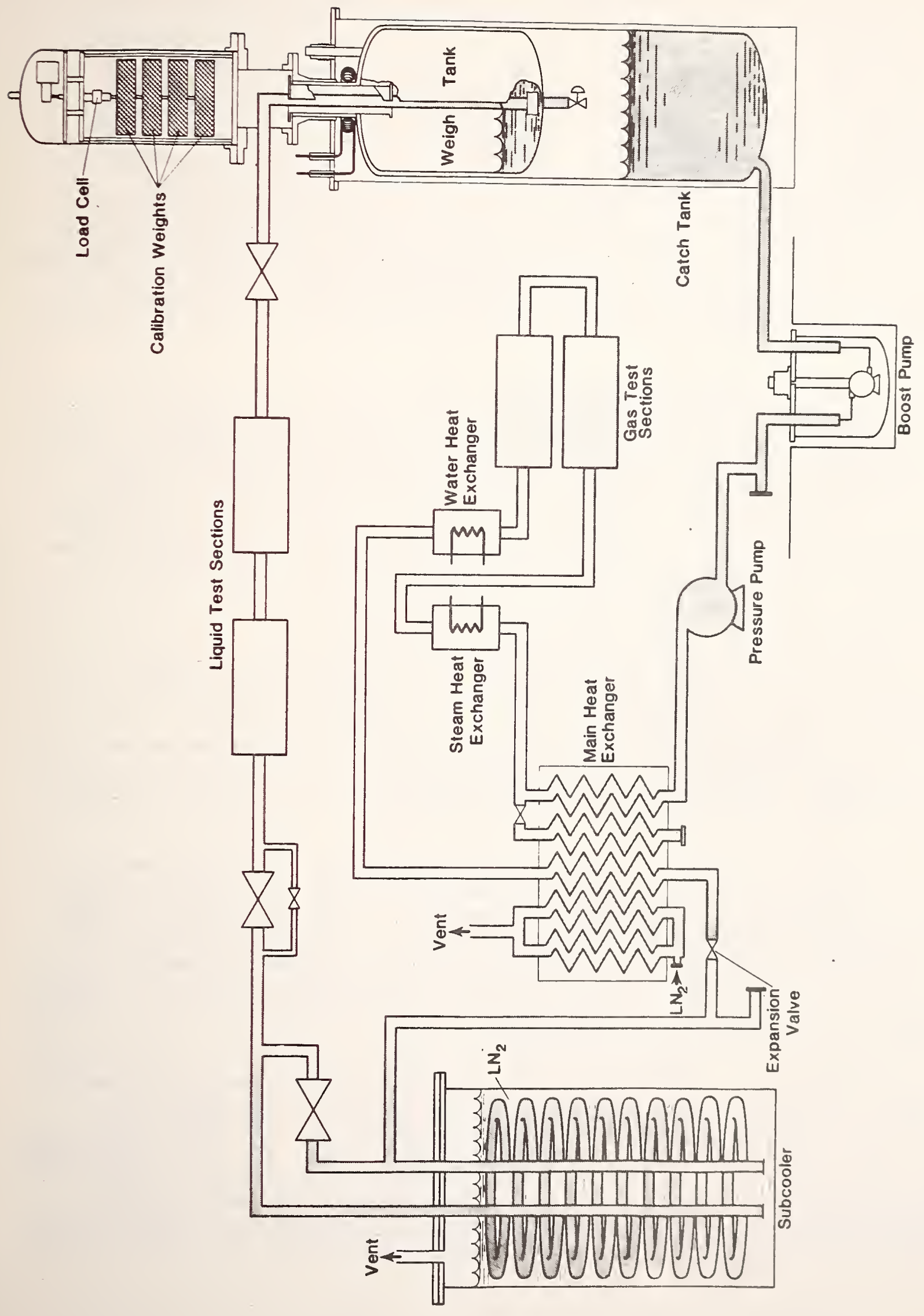

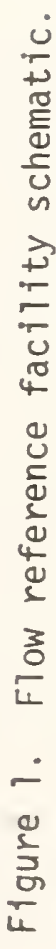


A11 of the instruments on the Gas Mass Flow Reference System were electrical devices except several pressure gauges that were used to monitor the system during start up. The electrical instrumentation was read by two small computers which were dedicated to the system and were on line during the system operation. One computer accessed instruments in the low pressure, cryogenic 1 iquid portion of the system (computer A). The other computer was used primarily but not exclusively to control and access instruments on the high pressure, gas portion of the system (computer B). Both computers operated in real time and interrogated the instruments at specified intervals or events. The signals read by the computers were converted to engineering units and were displayed at the computer terminals and/or stored on a mass storage device such as a magnetic tape or disk.

\subsubsection{Instruments for Measurement of Mass Flow}

Instrumentation input to computer $A$ included the liquid nitrogen temperatures and pressures, voltage from the load cell in the catch tank, the excitation voltage to the load cell and the test draft time. The load cell voltage and the excitation voltage to the load cell were read with digital voltmeters. The pressure and temperature sensors were read with a data logger. The data logger and the digital voltmeters were read by computer $A$, which then calculated the mass flow rate and recorded these data on magnetic tape.

\subsubsection{Instrumentation on the Gas Portion of the System}

All of the instruments on the warm portion of the system and some of the instruments on the cold portion were read with computer $B$. In all 30 temperature sensors and 30 pressure sensors were interrogated twice a minute during the monitoring mode. Signals from these sensors were converted to engineering units and then displayed at the computer console which was a cathode ray tube (CRT). The majority of the instruments were used for monitoring the system operation and stability.

The signals from most of the pressure transducers were in the range of two to ten volts at the operating conditions and did not require signal conditioning. Low level signals from some of the pressure transducers and all of the temperature sensors required amplification prior to entering the computer multiplexer and digitizers. The amplifiers were all in a single chassis with four amplifiers per card; ten cards were used. Each amplifier had a fixed gain but the gain was changeable by replacing a plug-in module. Use of this type of system prevented inadvertent gain changes.

Al1 signals were digitized and the computer converted them to engineering units by applying the appropriate mathematical equations. The polynomial equations were determined by calibrations. The pressure transducers were calibrated using a laboratory quality bourdon tube gauge. The temperatures were calculated using either the data from the Thermocouple Reference Table based on IPTS68 [5] or the calibration data from the manufacturer of the resistance thermometers. A precision constant current source controlled to 1 part in 7500 supplied the current for the resistance thermometers. 
The instruments used for the gas flow measurement with the orifice meters were calibrated separately. These instruments and the method of calibration will be discussed in Section 3 .

\subsection{Automatic Data Acquisition}

The two separate computers mentioned in Section 2.2 provided the automatic data acquisition, data display and data storage. Computer B had an input/output ( $I / 0)$ bus that accommodated cards with 16 input channels per card. Five of these cards were used as input channels for the 30 pressure and 30 temperatures discussed in Section 2.2. Each of these cards has a digitizer and each channel can be accessed individually or in series with the rest of the card. For calibration, the channels were individually selected and the analog signal was digitized and fed to the computer where it was incorporated as input for the instrument calibration.

During system operation, it was necessary to monitor all of the system functions so all data channels were interrogated in sequence $v i a$ the multiplexing capability at the I/O bus. The input signals were digitized as they were selected and this number was stored in an array. The number was then retrieved and each parameter was calculated using the polynomial equations determined by calibration.

The computer B was programmed to operate in three modes. The modes of operation were 1) monitoring the pressures and temperatures, 2) monitoring pressures and temperatures and storing the information on a disk and 3) taking flow test data during a test point. In the monitoring and storage mode computer B stored data on a magnetic disk in engineering units. Storage of these data facilitated investigation of system functions prior to test data points if desired. In the test point mode, data were also stored on a magnetic disk, but most of the data were stored as raw data signals from the sensors and their signal conditioning units, i.e., data were stored as digitized raw data. The program operated in the monitoring mode until the system stabilized at the desired flow rate. The test point mode was then selected. The computer read a barometer and accessed the data disk. It then informed the operator $v i a$ the CRT that it was prepared to take test data.

Computer A interrogated a data logger and two digital voltmeters. The data logger read the signals from pressure and temperature sensors in the liquid nitrogen. It also read the current supplied to the resistance thermometers. The two digital voltmeters read the load cell and the load cell excitation voltage. The actual starting point for the test was determined by a low point setting on the load cell reading. When the output from the load cell reached this set point, a custom-built electronic unit started the recording system. When the load cell reading reached a preset upper value, the unit stopped the recording system to end the test point. The catch tank was allowed to partially fill before the test point started thereby eliminating the initial disturbance caused by valve closure. The overall cycle was started by manual switch selection.

During the test draft computer $B$ repetitively interrogated four differential pressures, a static pressure and two temperatures and stored these as raw digital data on the magnetic disk. Two of the differential pressures 
were measured at the orifice meter; the other two were for monitoring the system stability during the test draft. The static pressure was measured at the downstream orifice pressure tap. The temperatures were measured at the orifice meter and in the liquid nitrogen upstream of the catch tank.

At the conclusion of the test draft, computer B calculated the mass flow rate through the orifice meter using ANSI/API 2530 [6]. This calculation was made using the square of the average of the square roots of the differential pressure readings made during the test draft. The average of the static pressure and the temperature were used to calculate the density of the nitrogen near the orifice plate. The mass flow rate at the orifice and the barometric pressure were transmitted to computer A. Computer A transmitted back to B the test point number and the time of day. The test point number with the time was used to identify the data on both computer mass storage units.

Computer A stored the test information on a magnetic tape. Computer B stored the raw data on a disk. At the conclusion of the calculation, the test point, date and time were stored along with the averages of the pressures and temperatures. The data on the tape of computer A was transferred to computer B and added to the data disk at the end of the day. The disk now contained all of the test point data for a particular test. These data were transferred to a large main frame computer where the raw data were again converted to engineering units and averages were calculated. The averages were compared to those calculated in computer B. The comparison was made as a check on the accuracy of data transmission.

Data in SI units were eventually written to the archival file. These data were also further reduced and coefficients were calculated, plots were generated, etc. from the engineering unit data file.

\subsection{Operation}

The time integrated mass flow rate passing through the test meter must be the same as the mass accumulated in the weigh tank. Instrumentation has been developed and applied to actively control and measure parameters which are sensitive to changes in this flow stability, and provide a measure of error resulting from non-stable flow.

The result of non-stable flow could be an error in the measurement by the reference system. This error would consist of an amount of mass of nitrogen fluid which is either measured by the test meter and not measured by the reference system or not measured by the test meter but measured by the reference system. The error could only result from a mass of fluid nitrogen either being stored or expelled in the volume which connects the test meter with the reference system.

For each flow test configuration, the volume of piping between the test meter and the reference system remains constant. There will be no change in the physical volume during the test draft period. Any measurement error must result from a change in fluid density at some point within the fixed volume between the test meter and the reference system.

Referring to Figure 1 , it is possible to isolate three separate subvolumes between the test meter and the reference system. The first subvolume is the ambient temperature volume measured from the active test meter element to the warm end of the main heat exchanger. A change in density within this volume can 
be found by measuring the temperature and pressure at the beginning and end of the test draft period and calculating the density change from the state equation of nitrogen [7].

Subvolume two is bounded by the warm end of the main heat exchanger and the low temperature expansion valve. It can be monitored for change in pressure using the same method as for subvolume one. Change in temperature of this volume is considered a change in bulk temperature because of the large temperature difference throughout the exchanger length. Measurement is done by recording the change in pressure within the capped heat exchanger flow passage and calculating an equivalent temperature change for the period of the test draft. The state equation of nitrogen is used to calculate this temperature change and to calculate the density change in the flow passage volume.

The third subvolume is that defined by the expansion valve and the weigh tank. This volume is filled with compressed single phase liquid nitrogen and can be directly monitored for change of pressure and temperature at the beginning and end of the test draft. Density is calculated from the state equation.

During each test, the above three subvolumes are monitored for pressure and temperature change. Density changes for each subvolume are calculated and added to give the total estimated error in terms of mass. This value is then in turn compared to the mass of the test draft and if found to be less than 0.1 percent the test point is considered stable. If found to be greater than 0.1 percent this fact was used only as indicator. The calculated error is not applied as a correction because the uncertainty of the error is greater than the estimated error of the flow measurements themselves. However, the procedure provides a measurable quantity which aids in defining and maintaining mass flow stability during the test draft period.

The above measurements are passive controls on the degree of flow stability during the test draft period. The results of the measurements and calculations provided limits to the pressure and temperature excursions tolerated by the experimentalist during the test draft period. As a result of these measurements made during the early part of the program, it was found that additional active control was needed to assure stable flow. Referring to figure 1, during the test draft period, the valve on the weigh tank is closed and the liquid level increases in the weigh tank and correspondingly decreases in the catch tank. A decrease in the level of the catch tank decreases slightly the suction head of the boost pump and results in a slight reduction in the pump output. This reduction is carried through the system as a slight decrease. in flow rate. For the fixed expansion valve setting, this reduction in flow gives a slight reduction in pressure at the gas test section. A control system was installed which increased the boost pump drive motor speed and pump output to compensate for the reduction in catch tank liquid level.

\section{ORIFICE METER GAS FLOW TESTS}

With pairs of each of the four line sizes and with six plates per meter run, it was possible to have 96 different combinations (not including repeat tests) of meter runs and plates. This number was reduced initially because of 
pressure differential and reference system flow restrictions. A test plan was generated which was designed to 1 imit the number of run and plate combinations based on meter performance. If a particular run-plate combination gave consistent results, then repeat runs were 1 imited. There were 856 test points included in the current test series. Of these test points, 17 were eliminated because of instrumentation or equipment failure during the test draft. The remaining 839 test points are included in this report. These test points represent 109 combinations including repeat tests and over 73,000 individual measurements of pressure, differential pressure and temperature.

\subsection{Orifice Flowmeter Test Equipment}

As part of the flow measurement program at NBS Boulder, the meter runs, the orifice plates and the flow conditioners were measured to document the dimensional data for record. Included with those measurement data are the dimensional data of a significant portion of the piping upstream of the meters in the NBS flow facility. The latter is also documented in an archival file [3] with the flow data corresponding to the installation.

\subsubsection{Orifice Meter Runs}

Each of the AGA/API meter runs is made up of three sections, an approach section, an upstream section and a downstream section. The approach section is affixed with a 600 psi ANSI flange on the upstream end and a special 0-ring sealed flange on the downstream end. The upstream section mates with this o-ring sealed flange on the approach section at the upstream end and has a similar 0-ring sealed flange on the downstream end which incorporates flange pressure taps built to the ANSI/API 2530 specification [6]. This flange is drilled in the bottom quadrants to accept two dowel pins which are used to position the orifice plate and align the runs. The upstream end of the downstream section mates with this flange and has corresponding flange taps and holes for dowel pins. The downstream end of this section is also equipped with a 600 psi ANSI flange. The flanges with taps have two tap holes, one at the top and one at the bottom. Figure 2 is a drawing of a typical flange at the orifice plate. The meter run diameter used for calculating data was the average of the diameters measured in the plain of the upstream flange taps. The meter runs were built to conform to the ANSI/API 2530 specification where applicable. A detailed description of the meter runs is given in Appendix 1 .

\subsubsection{Orifice Plates}

Two identical sets of six orifice plates were supplied with the meter runs. These plates were sized to cover a beta ratio (orifice diameter to pipe diameter) of about 0.2 to 0.75 . The plates are all nominally $1 / 8$ inch thick and have a 45 degree bevel on the outlet side. The plates are affixed with a paddle-type handle which is stamped with the identifying code and the nominal orifice size in inches. The plates were installed with the handle at the top for all of the tests. A detailed description of the plates and their identification is presented in Appendix 2. 


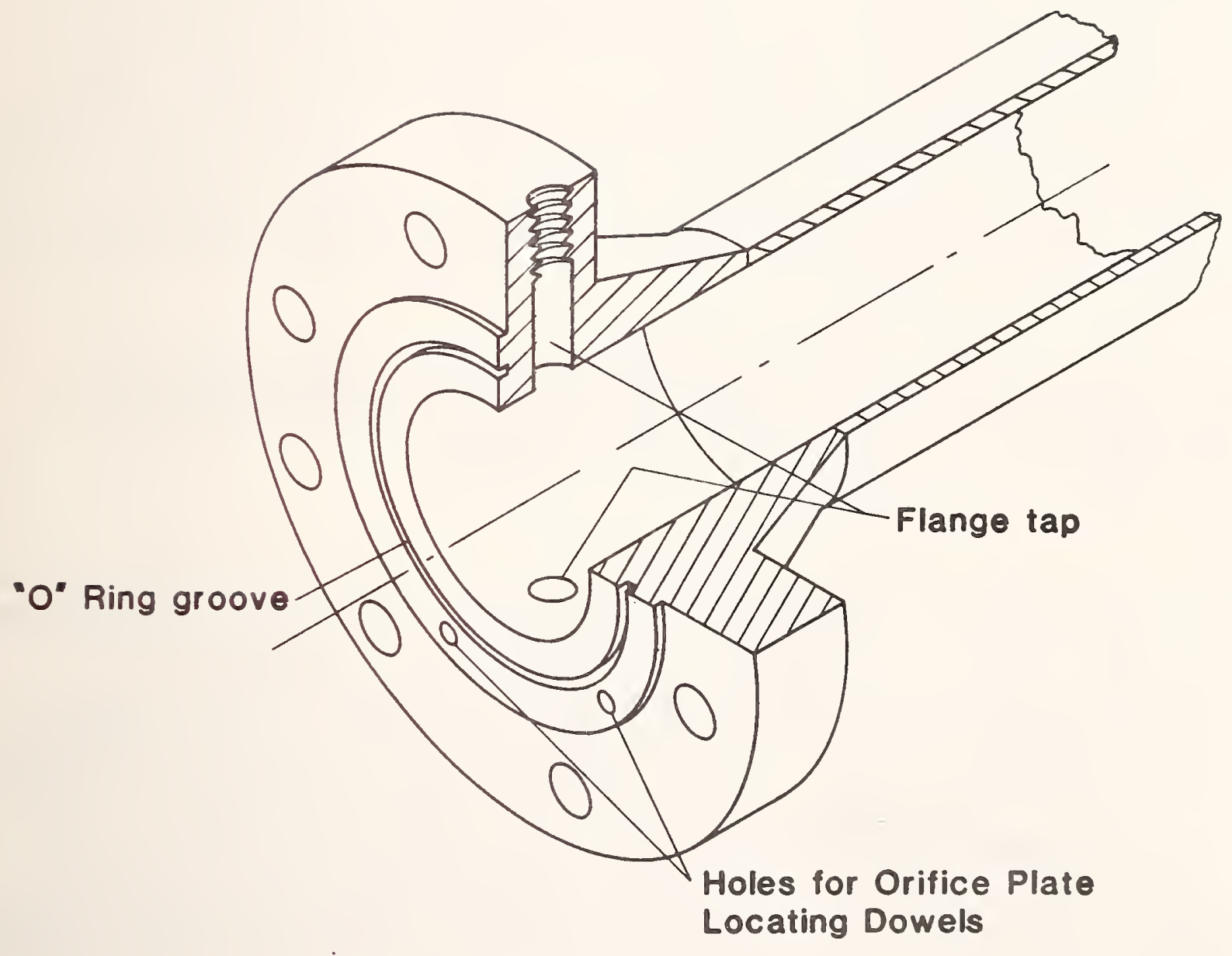

Figure 2. Typical orifice meter flange. 


\subsubsection{Fl ow Conditioners}

A perforated plate type flow conditioner $[8,9]$ was installed upstream of the approach section of the meter run during all of the tests recorded in the archival data file. Figure 3 shows the general layout of the plates and assembly. Three sizes of conditioners were used for the tests, a 4-inch, a 6 -inch and a 10-inch.

Usually the 4-inch conditioner was used with the 2-inch and 3-inch meter runs, the 6-inch conditioner with the 4-inch meter run and the 10-inch conditioner with the 6 -inch meter run. There were exceptions to this statement and they are documented in the archival data file and are apparent in the installation descriptions in Section 3.1.4. Since the flow conditioner housings were a larger pipe size than the meter runs, there were reducers on each end of the housing. The housings thus were constructed using a 600 psi ANSI flange welded to a reducer which was welded to a 600 psi ANSI flange the size of the housing. This flange mated to a similar flange which was welded to a length of run the nominal size of the unit. This straight run was welded to a reducer which was welded to a 600 psi ANSI flange the same size as the inlet flange. The 4-inch unit has 3 -inch inlet and outlet flanges; the 6-inch unit has 4-inch inlet and outlet flanges; and the 10-inch unit has 6-inch inlet and outlet flanges.

The perforated plates were attached to each other with spacer rods. The upstream plate was of the diameter to fit between the housing flanges thus securing it in the housing. The other two plates fit inside the housing. Al1 dimensions for the units are given in Appendix 3.

\subsubsection{Gas System Piping}

The portion of the NBS flow facility between the steam heat exchanger and the downstream end of the meter runs (see Figure 1) is described in detail here and in the archival data file [3]. From the steam heat exchanger the 4-inch pipe makes a right angle bend to the horizontal where it connects to the horizontal piping upstream of the meter runs. In general, the gas flow test section piping is in the form of a "U" shape in a horizontal plane containing (from the upstream flange) a 4-inch straight section and a 4-inch gas turbine meter with a 4-inch blocking valve and bypass. The bypass includes a 2 -inch block and bleed valve and 2-inch piping. Downstream of the bypass are two right angle elbows, a flow conditioner, the orifice meter and the gas test section downstream flange. The overall length of pipe depends on the nominal orifice meter length. Four-inch (unless otherwise specified) schedule 40 pipe spool pieces of various lengths are used to give equal length to the legs of the "U".

Six specific configurations were used. Each configuration used is identified in the archival data file. A schematic of the six configurations excluding the 2 -inch bypass is shown in Figure 4 . The following is a description of each configuration:

Test Piping Configuration Code 1981-A. Upstream flange +7.15 meter spool piece + gas turbine meter $(S / N 77-G-32 B)+4$-inch ball valve + 0.75 meter spool 

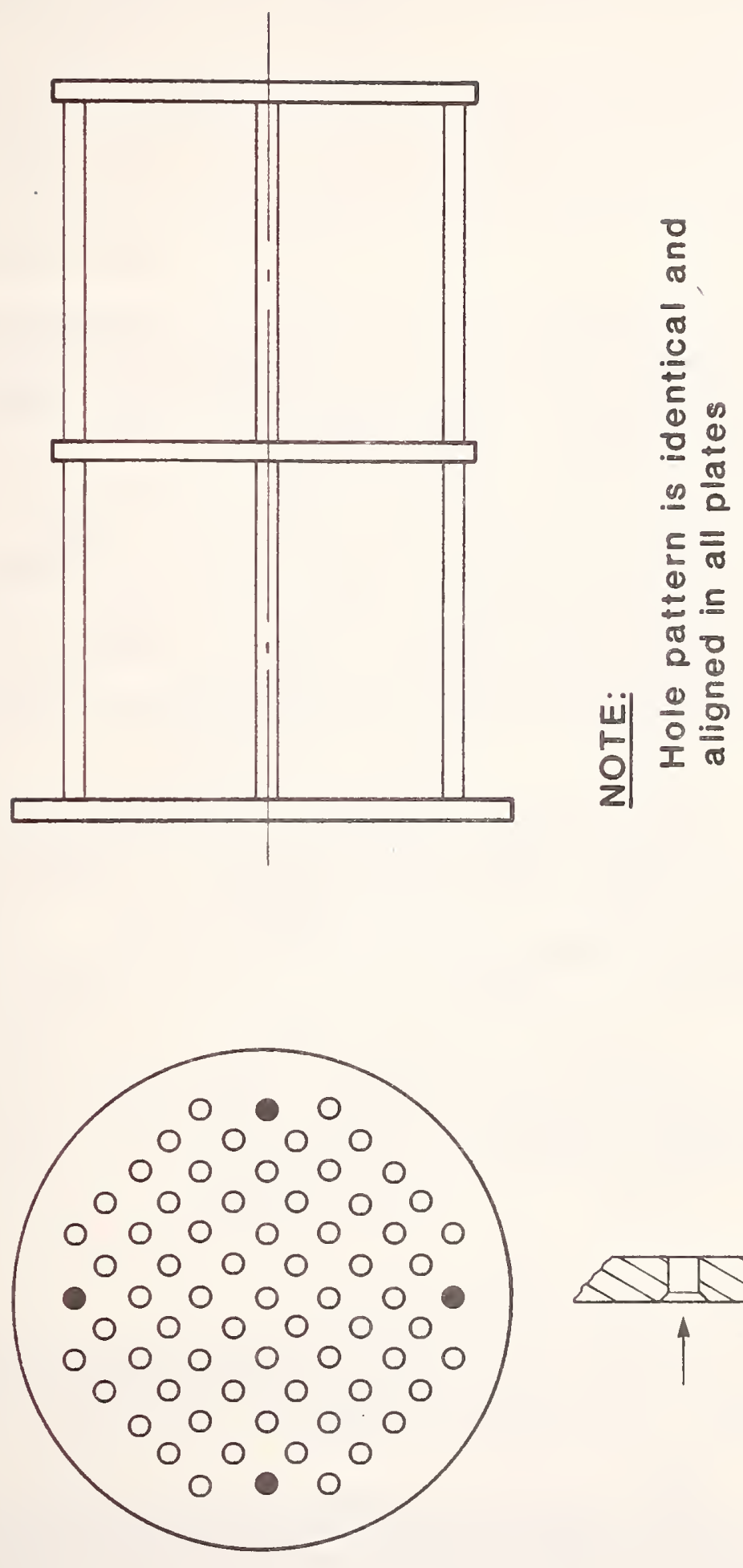

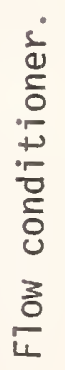

을

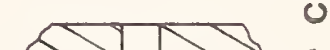

告

응

ำ 


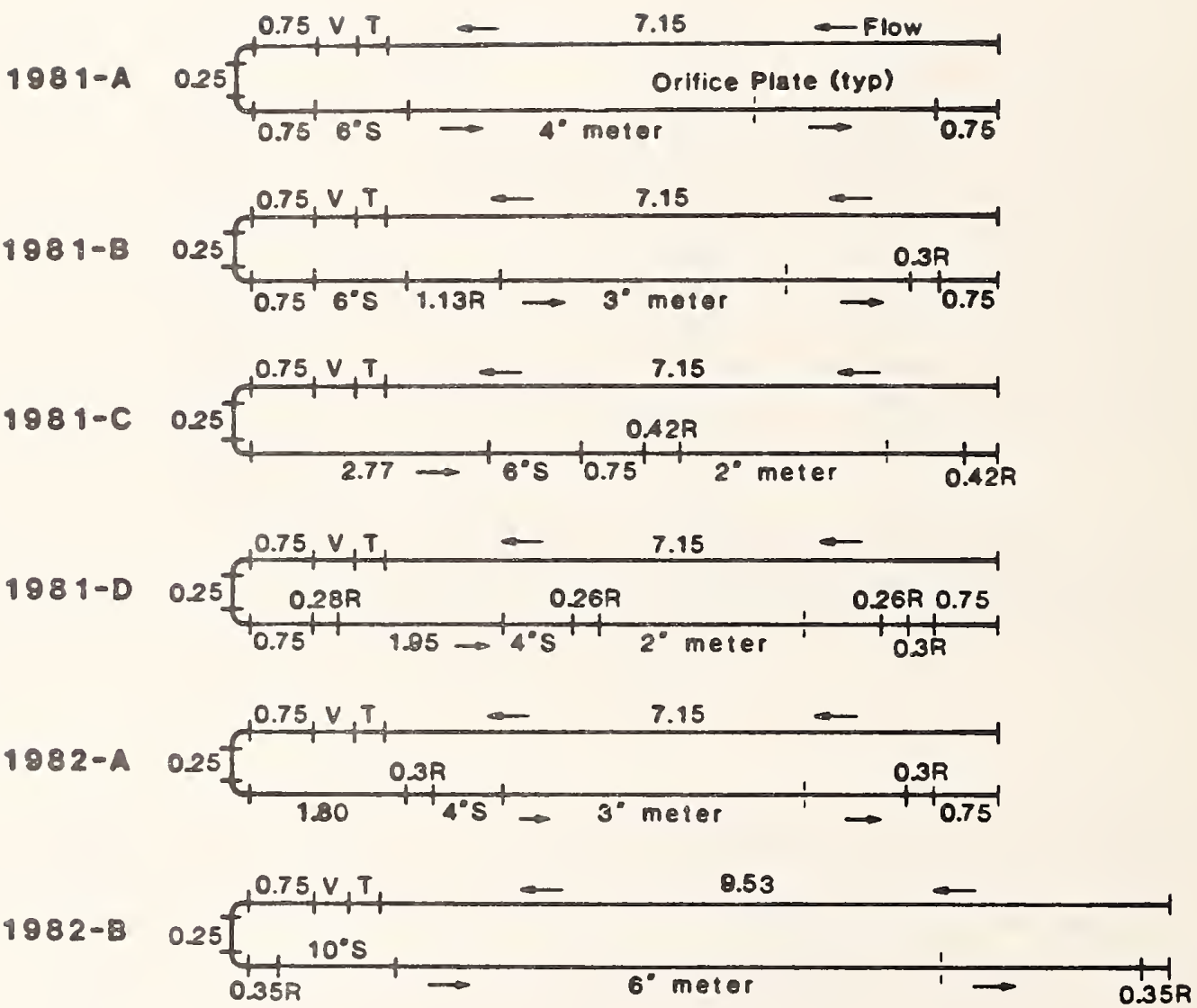

$$
\begin{aligned}
& S \text { - Flow conditioner } \\
& V-4 \text { inch ball valve } \\
& T-4 \text { inch gas turbine meter ( } / N 77-G-32 B \text { ) } \\
& R \text { - Reducing sections }
\end{aligned}
$$

NOTE:

All facility pipes are nominal 4 inch excepting two sections. The 1.85 meter section at 19810 and the 1.13R section at $1981 \mathrm{~B}$, which is a 4 to 3 inch reducer, are 3 inch pipe.

All dimensions in meters unless otherwise specified. Detailed dimensions of the meters are in Appendix 1.

Figure 4. Gas system piping schematic. 
piece + right angle elbow + 0.25 meter spool piece + right angle elbow +0.75 meter spool piece +6 -inch flow conditioner + AGA/API 4-inch orifice run +0.75 meter spool piece + downstream flange.

Test Piping Configuration Code 1981-B. Upstream flange +7.15 meter spool piece + gas turbine meter $(S / N 77-G-32 B)+4-i n c h$ ball valve +0.75 meter spool piece + right angle elbow +0.25 meter spool piece + right angle elbow +0.75 meter spool piece +6 -inch flow conditioner +1.13 meter reducer $(4 \times 3)+$ AGA/API 3 -inch orifice run +0.30 meter reducer $(3 \times 4)+0.75$ meter spool piece + downstream flange.

Test Piping Configuration Code 1981-C. Upstream flange +7.15 meter spool piece + gas turbine meter $(S / N 77-G-32 B)+4$-inch ball valve +0.75 meter spool piece + right angle elbow +0.25 meter spool piece + right angle elbow +2.77 meter spool piece +6 -inch flow conditioner +0.75 meter spool piece +0.42 meter reducer $(4 \times 2)+A G A / A P I 2$-inch orifice run +0.42 meter reducer $(2 \times 4)$ + downstream flange.

Test Piping Configuration Code 1981-D. Upstream flange +7.15 meter spool piece + gas turbine meter $(S / N 77-G-32 B)+4$-inch ball valve +0.75 meter spool piece + right angle elbow +0.25 meter spool piece + right angle elbow +0.75 meter spool piece +0.28 meter reducer $(4 \times 3)+1.95$ meter spool piece $(3$-inch $)+4$-inch flow conditioner +0.26 meter reducer $(3 \times 2)+$ AGA/API 2 -inch orifice run +0.26 meter reducer $(2 \times 3)+0.30$ meter reducer $(3 \times 4)+$ 0.75 meter spool piece + downstream flange.

Test Piping Configuration Code 1982-A. Upstream flange +7.15 meter spool piece + gas turbine meter ( $S / N$ 77-G-32B) + 4-inch ball valve + 0.75 meter spool piece + right angle elbow +0.25 meter spool piece + right angle elbow +1.80 meter spool piece +0.30 meter reducer $(4 \times 3)+4$-inch flow conditioner + AGA/API 3 -inch orifice run +0.30 meter reducer $(3 \times 4)+0.75$ meter spool piece + downstream flange.

Test Piping Configuration Code 1982-B. Upstream flange +9.53 meter spool piece + gas turbine meter $(S / N 77-G-32 B)+4$-inch ball valve +0.75 meter spool piece + right angle elbow +0.25 meter spool piece + right angle elbow +0.35 meter reducer $(4 \times 6)+10$-inch conditioner + AGA/API 6-inch orifice run + 0.35 meter reducer $(6 \times 4)+$ downstream flange.

\subsection{Orifice Meter Instrumentation}

The performance of orifice meters of defined geometry measuring a single component fluid requires three parameters; static pressure, flowing fluid temperature and differential pressure across the orifice plate. With these three parameters the mass flow rate was calculated from the known properties of the process fluid [7].

The automated data acquisition system described in Section 2.3 recorded the measurements from the orifice meter instruments. Electronic signals were supplied to the computer from pressure and temperature transducers and associated signal conditioning equipment. The static pressure and the differential pressure were measured with transducers whose output was current proportional to pressure. Current from each of the transducers passed through 
a precision resistor and the voltage across the resistor was read and digitized, then fed to the computer. The computer also recorded the voltage across the platinum resistance thermometer used to measure fluid temperature. All of the digitized voltage signals were recorded on mass storage disks and subsequently transmitted to a larger main frame computer where all data reduction, analysis and presentation were performed.

The differential pressure across the orifice plate was measured at the flange taps using two differential pressure transducers. The lower range transducer which was 0 to $25 \mathrm{kPa}$ was used until the range was exceeded, then the high range transducer 0 to $50 \mathrm{kPa}$ was automatically selected. Both of these transducers experienced small but significant changes in the zero signal. This change necessitated daily calibrations and use of the calibration for that days tests. The differential pressure transducers were calibrated using a cistern mercury manometer at a base pressure of $4.1 \mathrm{MPa}$ which was near the system operating pressure. This mercury manometer was calibrated at ambient base pressure with an air dead weight gauge certified by NBS. Corrections were made to the mercury manometer reading to adjust the ambient pressure calibration to the $4.1 \mathrm{MPa}$ base pressure. These corrections were verified by using two dead weight gauges, one for the base pressure and the other for the base plus the differential pressure. This technique and documentation of the accuracy of the pressure calibration facility has been reported by sindt and LaBrecque [10].

The 640 individual differential pressure calibration data points were fitted to a straight line. Statistical analysis show a residual standard deviation of $22 \mathrm{~Pa}$ where as for any of the single calibrations using 12 points fitted to a straight line, the residual standard deviation ranged from 5 to $15 \mathrm{~Pa}$. The total uncertainty 7 imit of any single reading of these transducers is predicted to be 38 to $54 \mathrm{~Pa}$ depending on the reading. The total uncertainty limit for 30 measurements, the minimum taken per test point, is estimated to be $0.07 \%$ at $50 \mathrm{kPa}, 0.1 \%$ at $25 \mathrm{kPa}$ and $0.7 \%$ at $2.5 \mathrm{kPa}$. This total uncertainty limit includes the uncertainty contributed by the transducers, the mercury manometer and the dead weight gauge.

The static pressure was measured at the downstream orifice flange tap using a transducer with a range of 0 to $6.895 \mathrm{MPa}$. This transducer was calibrated daily using an air dead weight gauge that was certified by NBS [10]. Nine hundred and thirty-five calibration data points from this transducer were fitted to a straight 7 ine. The resulting residual standard deviation from this fit was within $\pm 0.12 \%$ at the $4.1 \mathrm{MPa}$ operating pressure.

The gas temperature was measured downstream from the end of the orifice meter runs using a platinum resistance thermometer driven by a current source which maintained a constant current within $+0.01 \%$. The thermometer was calibrated by comparison with a transfer ständard certified by NBS. Comparisons were made at the water ice point and at points up to $37 \mathrm{~K}$ above the ice point. From these comparisons the estimated uncertainty of the thermometer readings was $-0.26 \mathrm{~K}$ to $+0.15 \mathrm{~K}$ for one reading; for thirty readings the estimated uncertainty was $-0.12 \mathrm{~K}$ to $+0.0 \mathrm{~K}$. The procedure for calibration was to first check the water ice point reading of both the thermometer and the transfer standard, then comparisons were made at about six points up to $310 \mathrm{~K}$. The ice point was again checked as the last reading. 
For all of the meter installations shown on Figure 4, the thermometer was located $0.6 \mathrm{~m}$ downstream from the last flange indicated on the schematic. The distance from the orifice plate to the thermometer was the length of the downstream meter section plus any spool piece and reducer shown on Figure 4 plus $0.6 \mathrm{~m}$.

\subsection{Flow Test Procedures}

As previously stated tests were conducted on two sets each of four sizes of meter runs. Each meter run is identified as FE1 through FE8 and includes unstream and downstream piping. Each meter run had six pairs of orifice plates covering a range of beta ratios of approximately 0.2 to 0.75 . Plates of the same nominal size were identified with the same numeric value from 1 to 6 . Individual plates of the same size were further identified with a suffix of $A$ or B.

For the tests reported here the orifice pressure differential was limited to the range 2.5 to $50 \mathrm{kPa}$. The flow facility has a gas flow range limitation of 0.45 to $2.5 \mathrm{~kg} / \mathrm{s}$. These two 1 imitations precluded testing all the orifice plates in any meter size. For a variety of other reasons not all plate-meter run combinations were tested. Table 1 lists the orifice plate-meter run combinations that were available. An " $x$ " in the body of the table indicates the combinations that were tested.

Table 1. Orifice Plate-Meter Run Test Combinations

\begin{tabular}{|c|c|c|c|c|c|c|c|c|c|c|c|}
\hline Run/Orifice & $1 \mathrm{~A}$ & $1 B$ & $2 A$ & $2 B$ & $3 \mathrm{~A}$ & $3 B$ & $4 A$ & 4B & $5 A$ & 5 & \\
\hline $\begin{array}{l}\text { FE1 } \\
\text { FE2 }\end{array}$ & & & & & & & $\begin{array}{l}x \\
x\end{array}$ & $\begin{array}{l}x \\
x\end{array}$ & $\begin{array}{l}x \\
X\end{array}$ & & $x$ \\
\hline FE3 & & & $x$ & $x$ & & $x$ & & $x$ & $x$ & & \\
\hline FE4 & & & $x$ & $x$ & $x$ & $x$ & $x$ & $x$ & $x$ & & $x$ \\
\hline FE5 & & & $x$ & $x$ & $X$ & $x$ & $x$ & $x$ & $x$ & & $x$ \\
\hline FE6 & & & $x$ & $x$ & $x$ & $x$ & $x$ & $x$ & $x$ & & $x$ \\
\hline EE7 & $x$ & X & $X$ & $x$ & $x$ & $x$ & $x$ & $x$ & & & \\
\hline - & $x$ & $x$ & $x$ & & $x$ & $x$ & $x$ & $X$ & & & \\
\hline
\end{tabular}

The test plan used in this study was randomized in flow rate, plate selection and run selection. A series of randomized flow rate tests were conducted for each orifice plate-meter run combination. The method of taking data with continuously increasing or decreasing flow rates was not used. Repeated flow rate points were also randomized. With one exception, repeated tests with a given orifice plate were not done without at least removing and replacing the plate in the meter run. Normally, repeated tests were not done consecutively. 
The data were analyzed by calculating the product of the discharge coefficient, $C$, and the expansion factor, $Y_{2}$, and plotting the results as a function of the bore Reynolds Number. It was not possible to separate the values of $C$ and $Y_{2}$ in this phase of the test program although tests are underway which will allow this to be done.

The $\mathrm{CY}_{2}$ product was calculated using equation (1).

$$
C Y_{2}=\frac{(\text { constant })(\dot{m})\left(1-e^{4}\right)^{\frac{1}{2}}}{\left(F_{a}\right)\left(d^{2}\right)(\rho \Delta P)^{\frac{1 / 2}{2}}}
$$

where $\mathrm{C}=$ discharge coefficient

$\mathrm{Y}_{2}=$ expansion factor based on downstream pressure

$\dot{\mathrm{m}}^{2}=$ mass flow rate

$B=$ diameter ratio or beta

$d=$ orifice diameter

$\mathrm{F}_{\mathrm{a}}=$ orifice plate thermal contraction factor [6]

$\rho \quad=$ gas density

$\Delta P \quad=$ orifice differential pressure

constant = numeric function of dimensions and conversion factors.

When the mass flow rate is in units of $\mathrm{kg} / \mathrm{s}$, orifice diameter is in units of $\mathrm{cm}$, gas density is in units of $\mathrm{kg} / \mathrm{m}^{3}$ and differential pressure is in units of $\mathrm{kPa}$, then the

$$
\text { constant }=\frac{(4)\left(10,000 \mathrm{~cm}^{2} / \mathrm{m}^{2}\right)}{\pi((2) 1,000 \mathrm{~Pa} / \mathrm{kPa})^{\frac{1}{2}}}=284.705 \text { (SI units) }
$$

When the mass flow rate is in units of $1 \mathrm{~b}_{\mathrm{m}} / \mathrm{s}$, orifice diameter is in units of inches, gas density is in units of $1 \mathrm{~b}_{\mathrm{m}} / \mathrm{ft} \mathrm{t}^{3}$ and differential pressure is in units of inches of $\mathrm{H}_{2} \mathrm{O}$, then the

$$
\text { constant }=\frac{(4)\left(144 \mathrm{in}^{2} / \mathrm{ft}^{2}\right)}{\pi\left(\rho_{W} / 12\right)^{\frac{1}{2}}\left(2 \mathrm{~g}_{C}\right)^{\frac{1}{2}}}=10.0258 \text { (British units) }
$$

where $\rho_{W}=62.3664 \mathrm{ib} / \mathrm{ft}^{3}$; the density of water at $60^{\circ} \mathrm{F}(1 \mathrm{~atm}$.$) [11].$ $g_{c}=32.1741 b_{m} \cdot \mathrm{ft} / 1 b_{f} \mathrm{~s}^{2}$; the gravitational constant. 
The test identification is used to specify a single test or group of tests and is carried through all of the tables. The key for the test identification is as follows:

Test run at NBS-Boulder

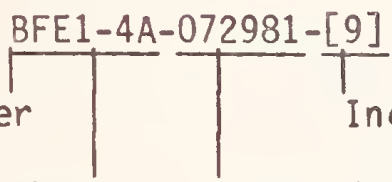

Individual test point number(s)

Meter run and plate number

Test date as MMDDYY

Tabular values for all values of the physical measurements of the orifice plates and meter runs available to this project are given in tables 2 (a), 3 (a), 4 (a) and 5 (a) in the International System of Units (SI). The same information is also provided in U.S. Customary Units (British) in tables 6 (a), 7 (a), 8 (a) and 9 (a). All measured parameters taken during the test program and all calculated values are tabulated in tables 2 (b), 3 (b), 4 (b) and 5(b) in SI units and in tables 6 (b), 7 (b), 8 (b) and 9 (b) in British units.

The number of significant digits shown in the tables are more than can be justified by the accuracy limitations but are carried for computational consistency. The differential pressures in these tables are based on a density of water at $288.71 \mathrm{~K}(101.325 \mathrm{kPa})$ as being $0.999014 \times 10^{3} \mathrm{~kg} / \mathrm{m}^{3}$ $[11]$.

$\mathrm{CY}_{2}$ and $\mathrm{KY}_{2}$ values were calculated for each of the "N" data samples. The tabulated values were determined by taking a simple average of the individual calculations for each test point.

All the $\mathrm{CY}_{2}$ values in the tables are plotted as a function of the reciprocal bore Reynolds Number in figures 5 through 8 . Also included in the figures are curves denoting the values of $\mathrm{CY}_{2}$ that were calculated using the two methods specified by the ANSI/API 2530 and the ISO 5167 standards [12]. The value of $Y_{2}$ used in both curves was calculated as specified in the ANSI/API 2530 standard and is responsible for the upward curvature at high bore Reynolds Numbers.

From the figures it is apparent that the standards do not adequately predict experimental data for all line sizes and beta ratios. The data have size and beta ratio dependencies different from those predicted by the standards.

Figure 9 shows the relative relationship of the data presented here with the original water data [13] from which both standards were developed.

\subsection{Future $P l a n s$}

During 1984 and 1985, NBS plans to continue evaluation and analysis of these data under GRI sponsorship. The ultimate goal of this research is to establish an accurate relationship for measuring high volume gas flows using orifice meters. 


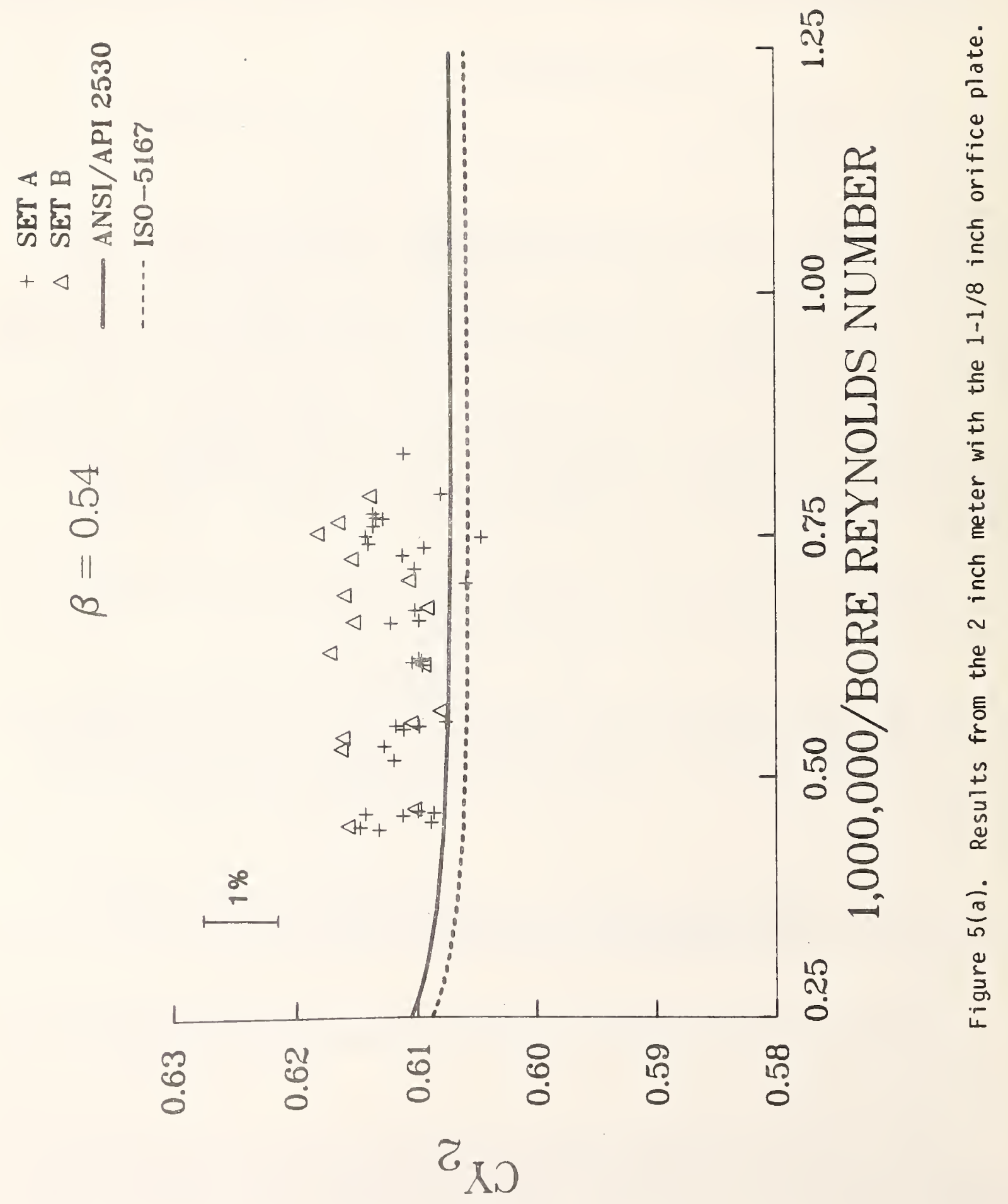




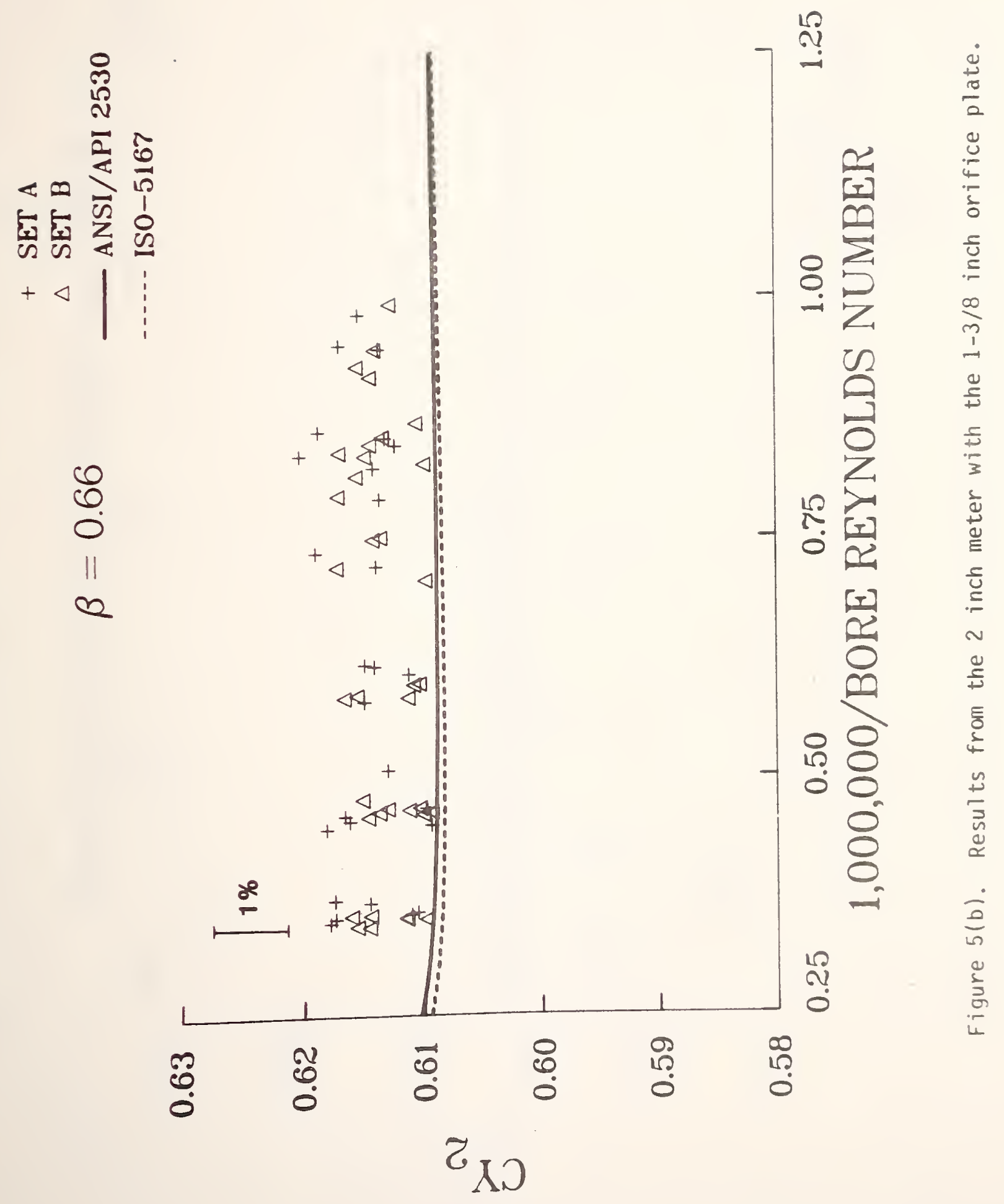




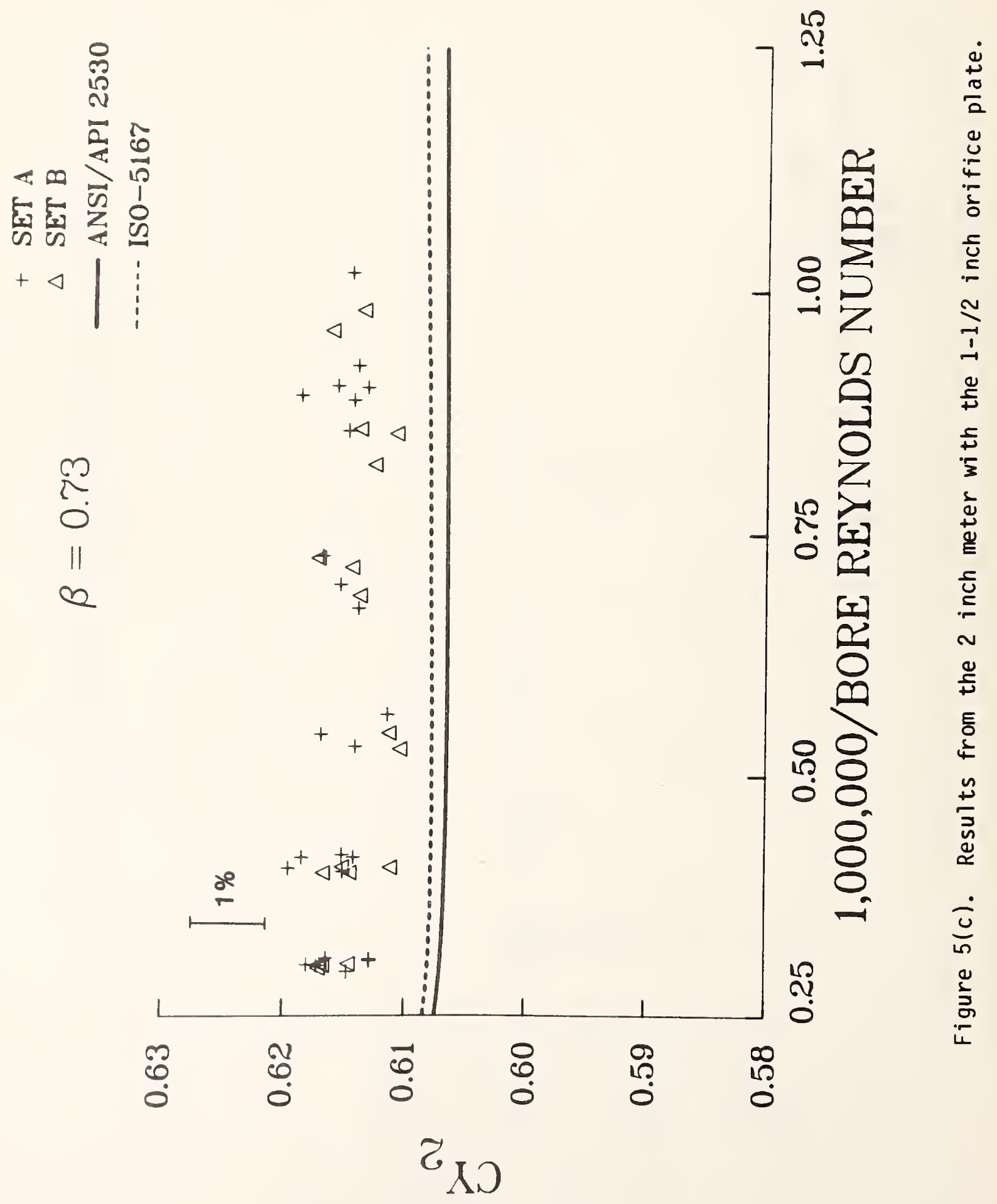




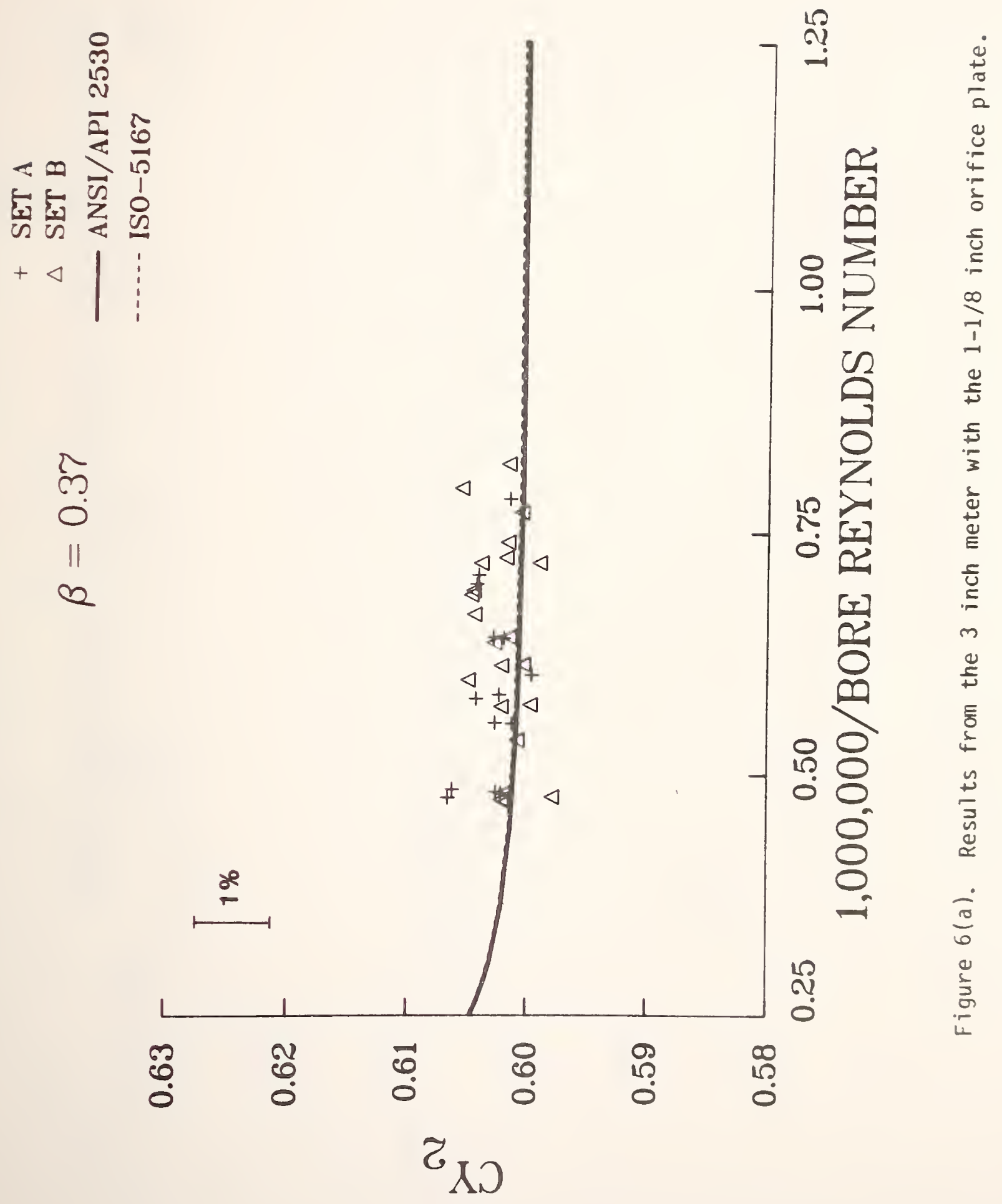




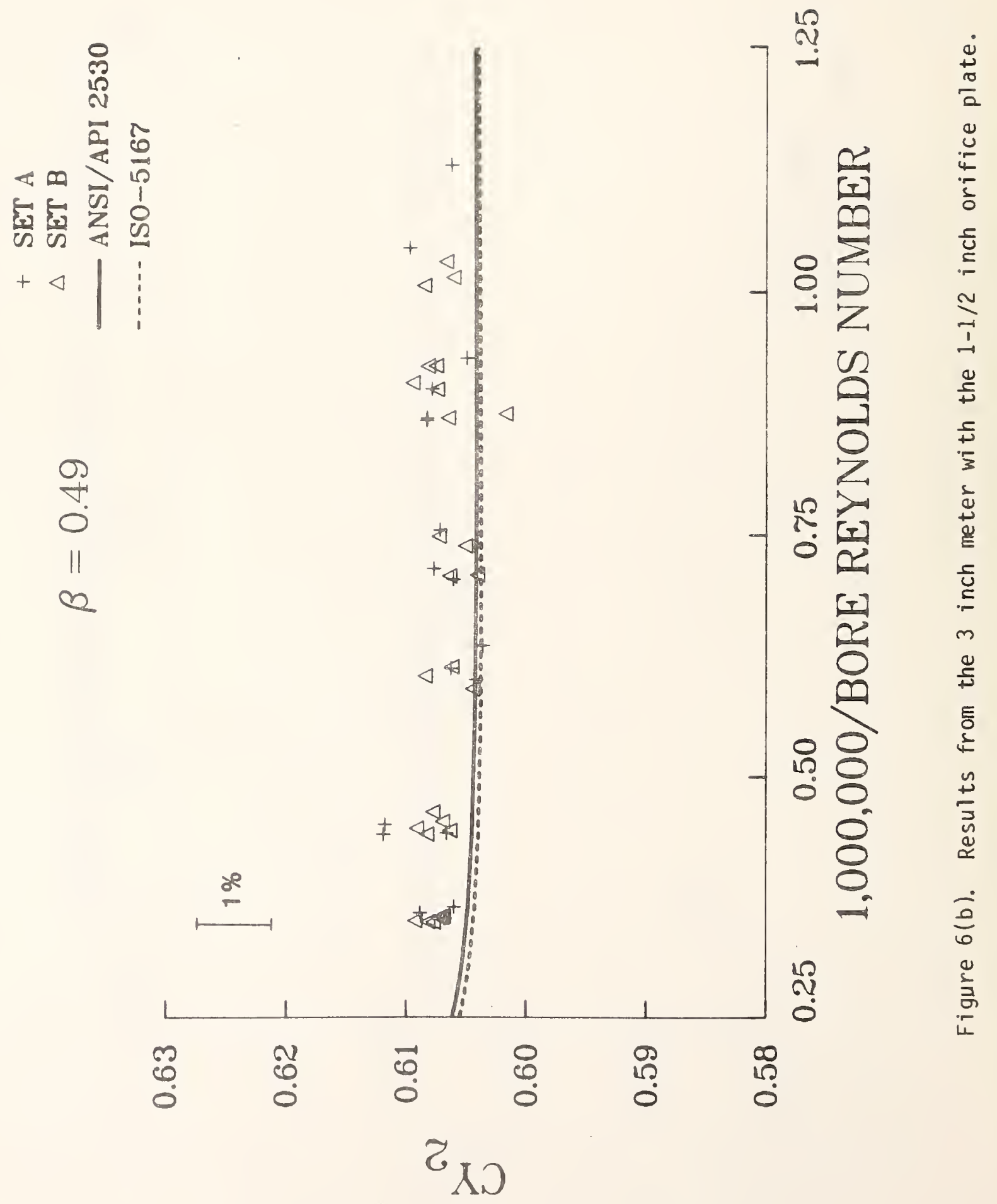




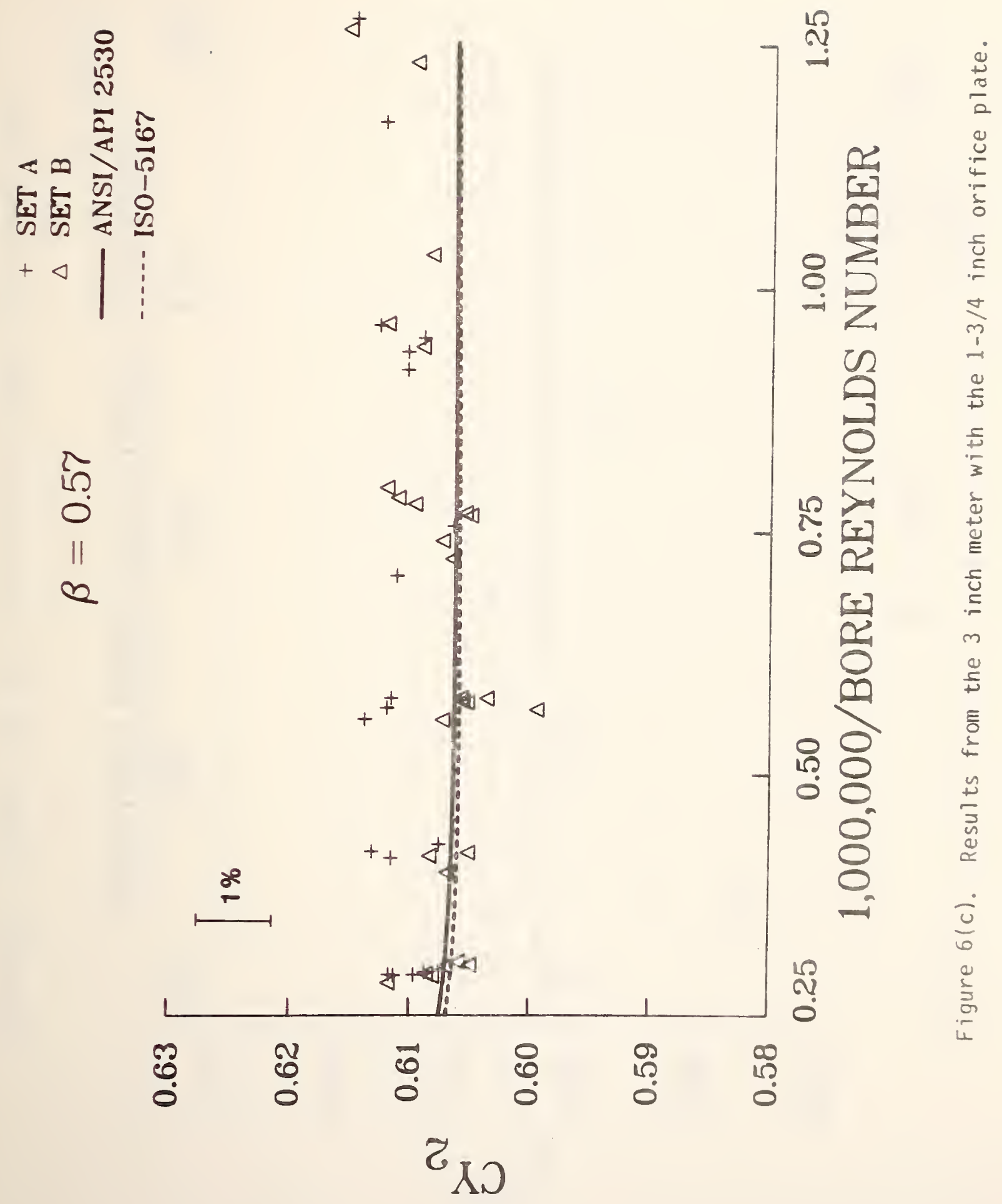




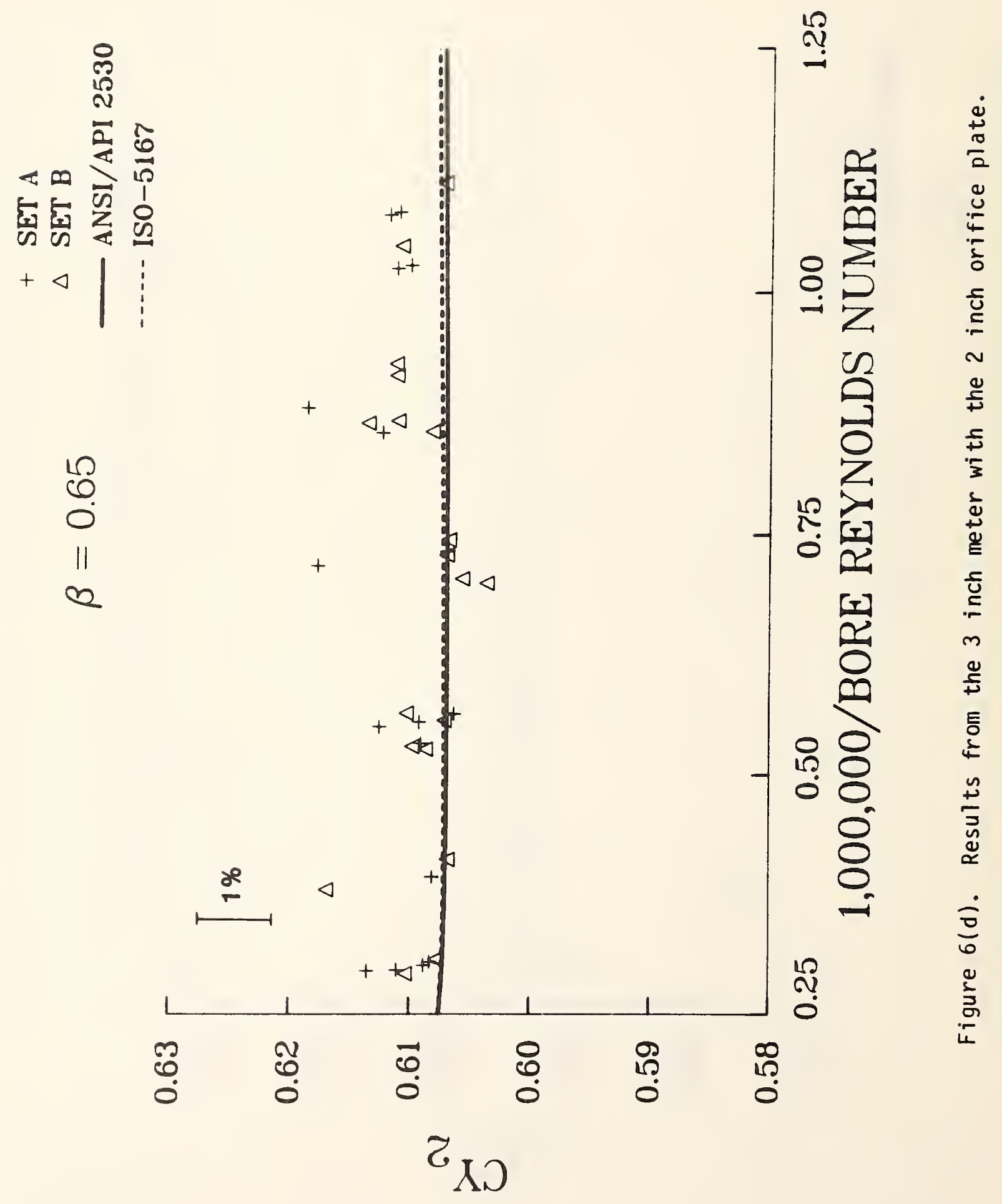




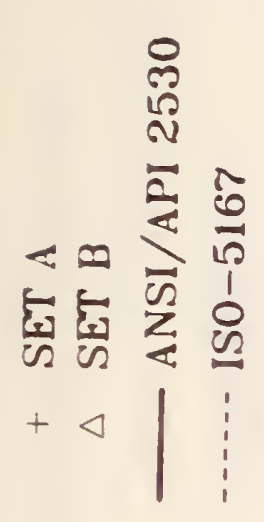

20
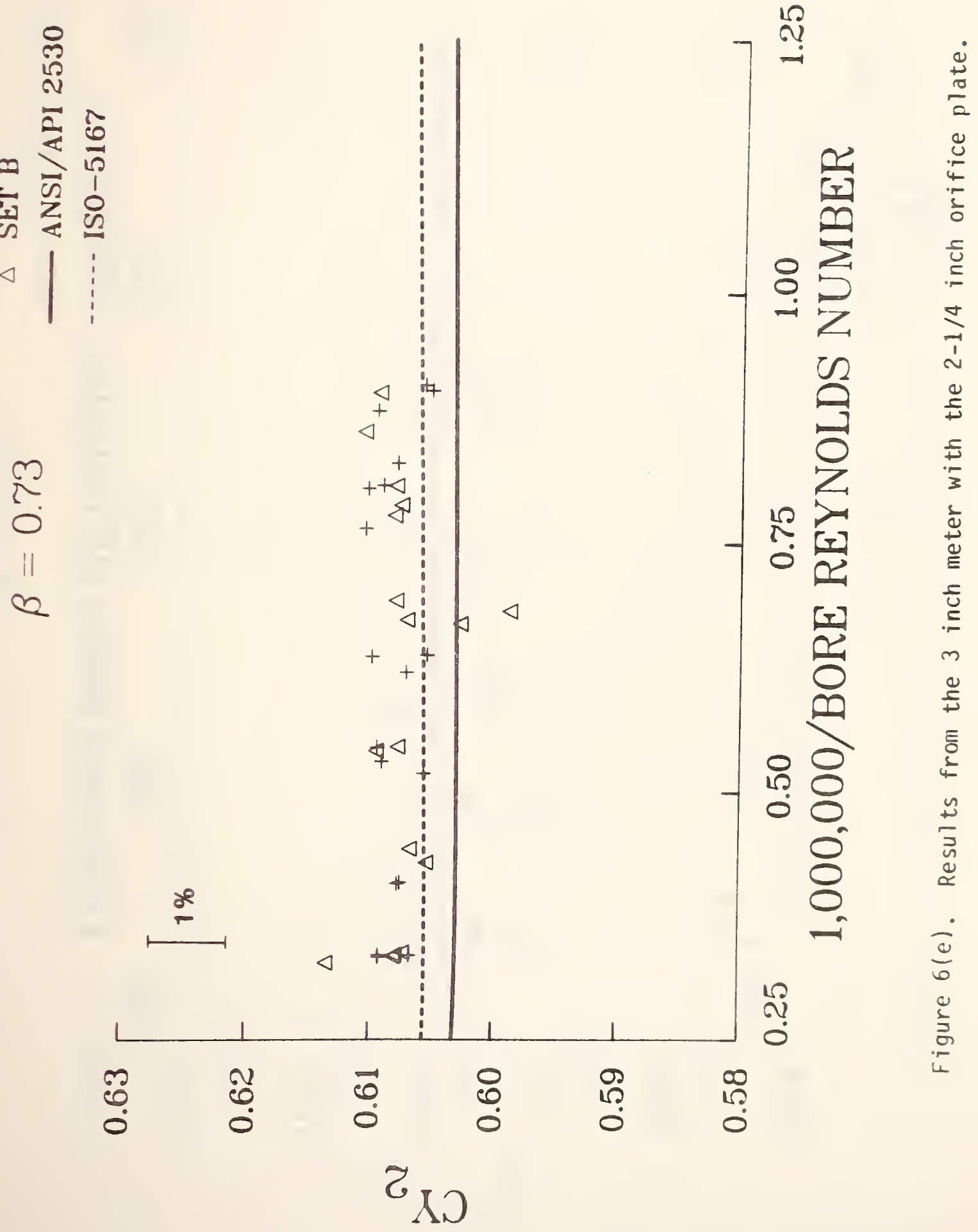


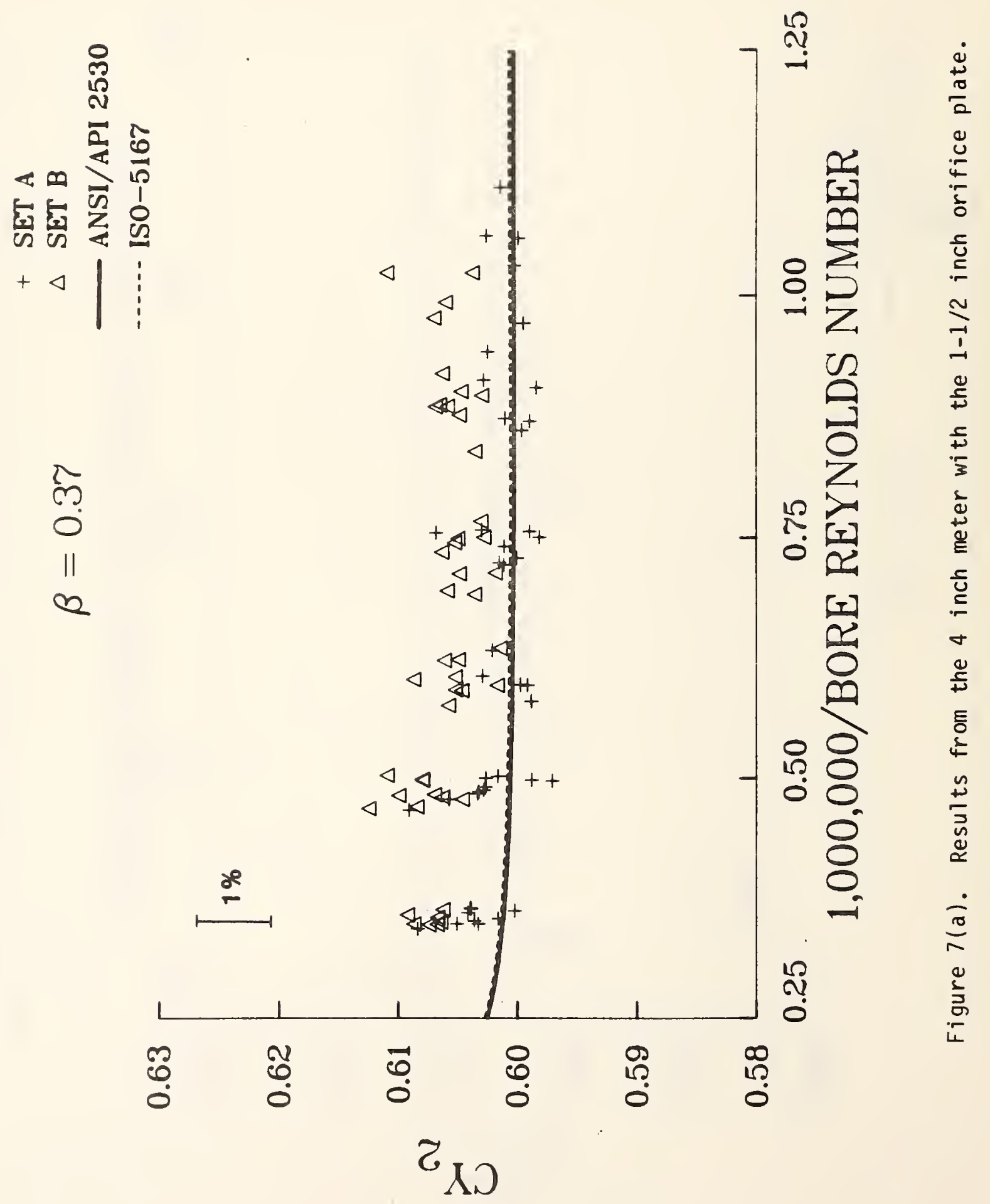




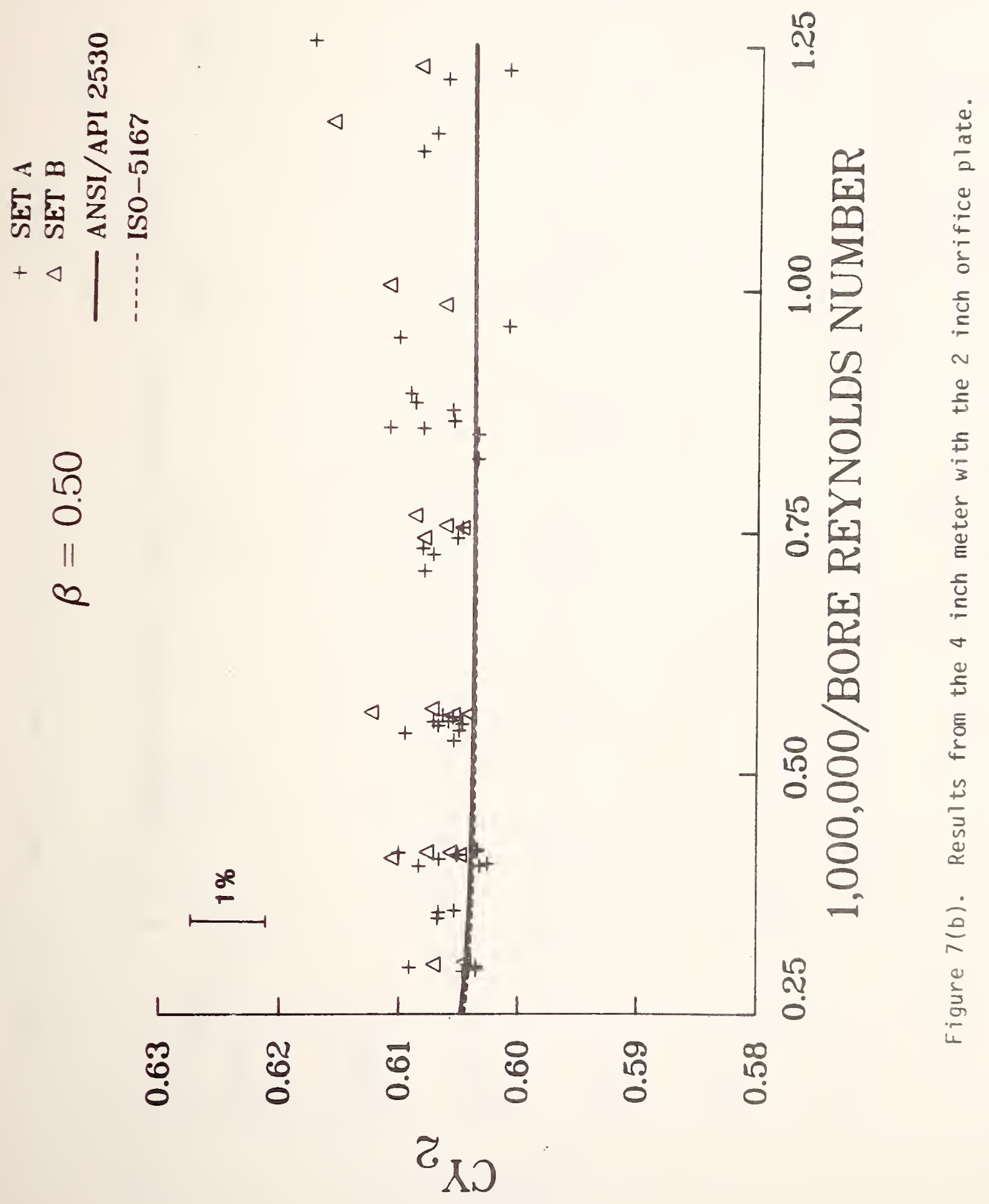




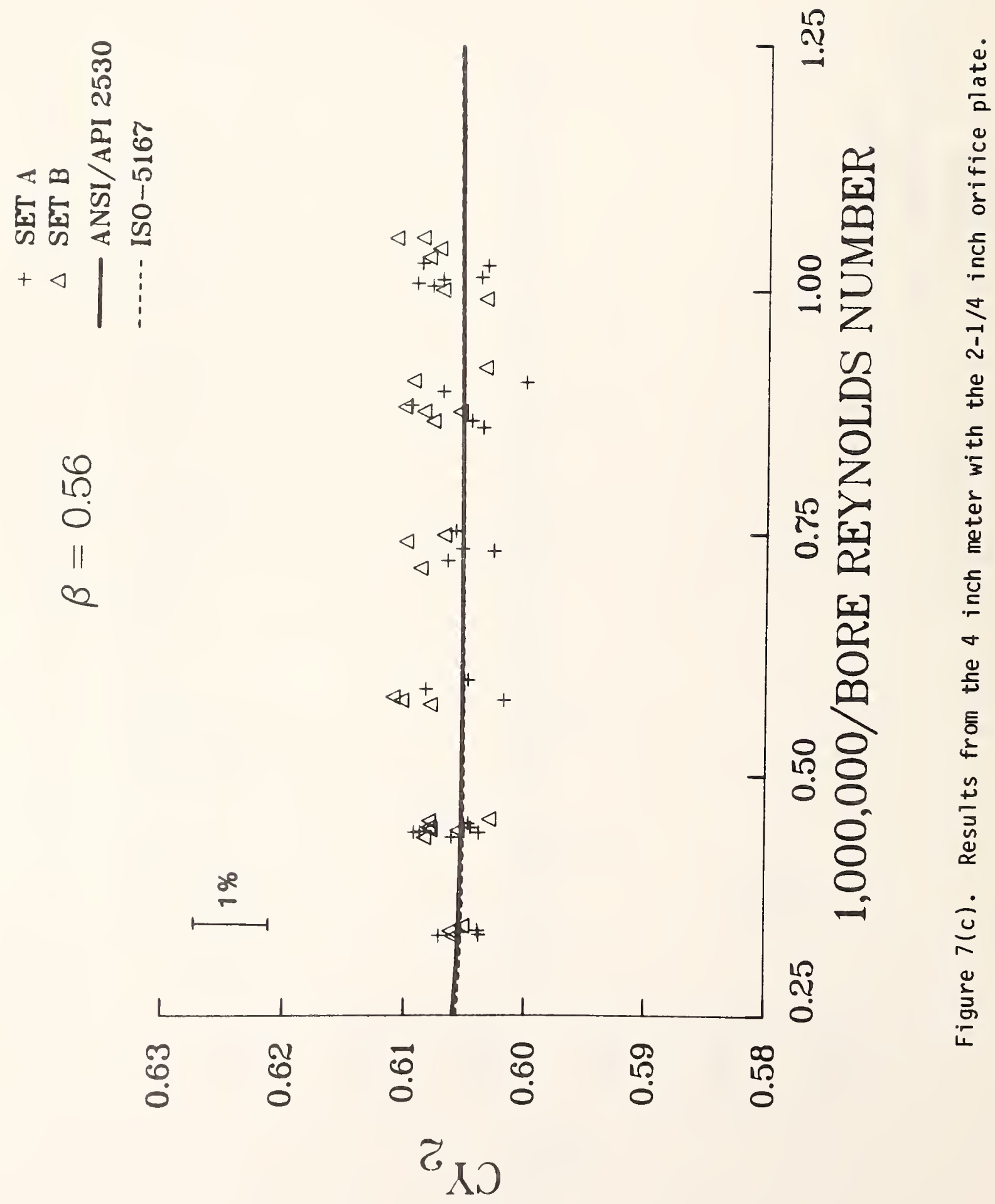




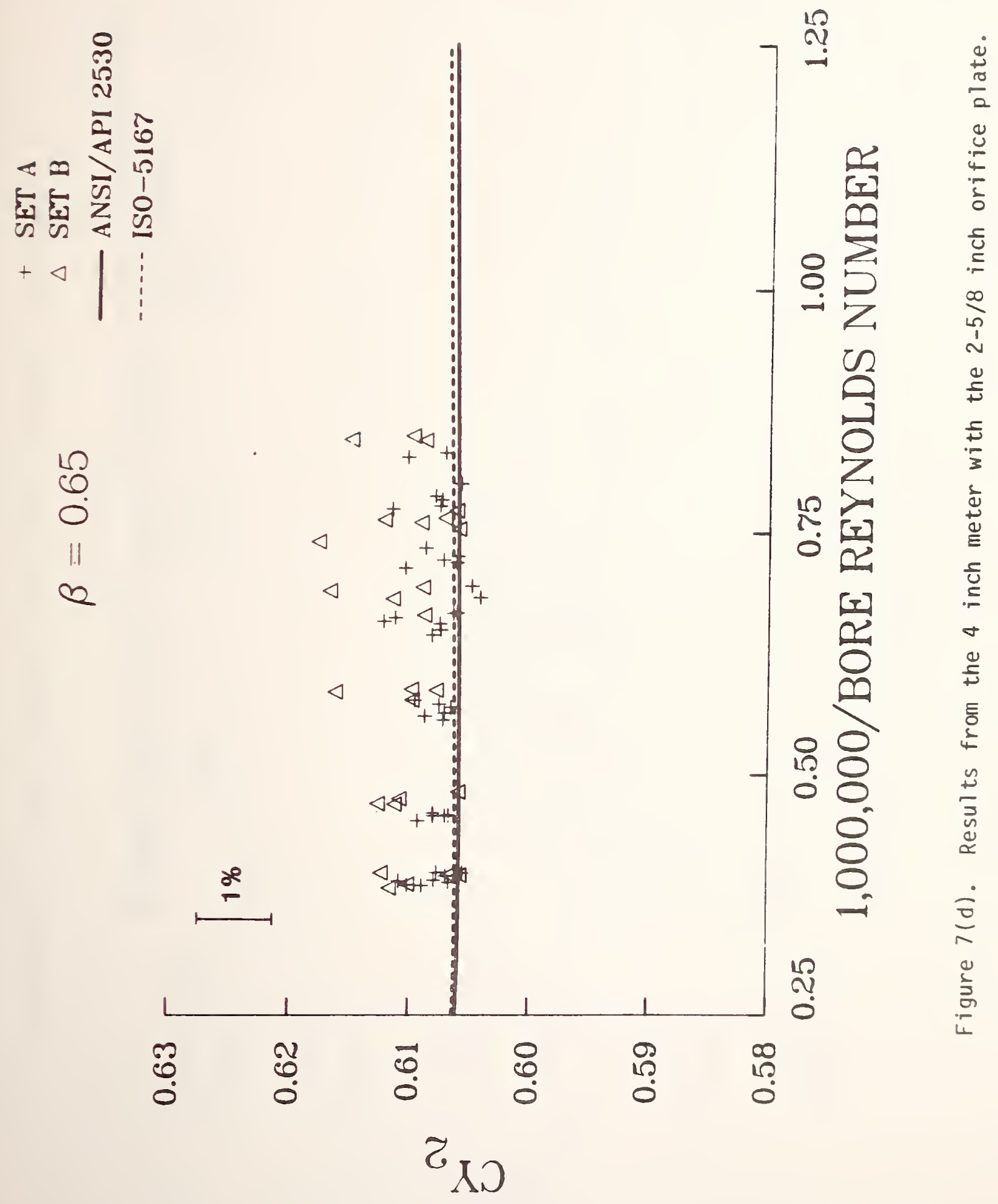




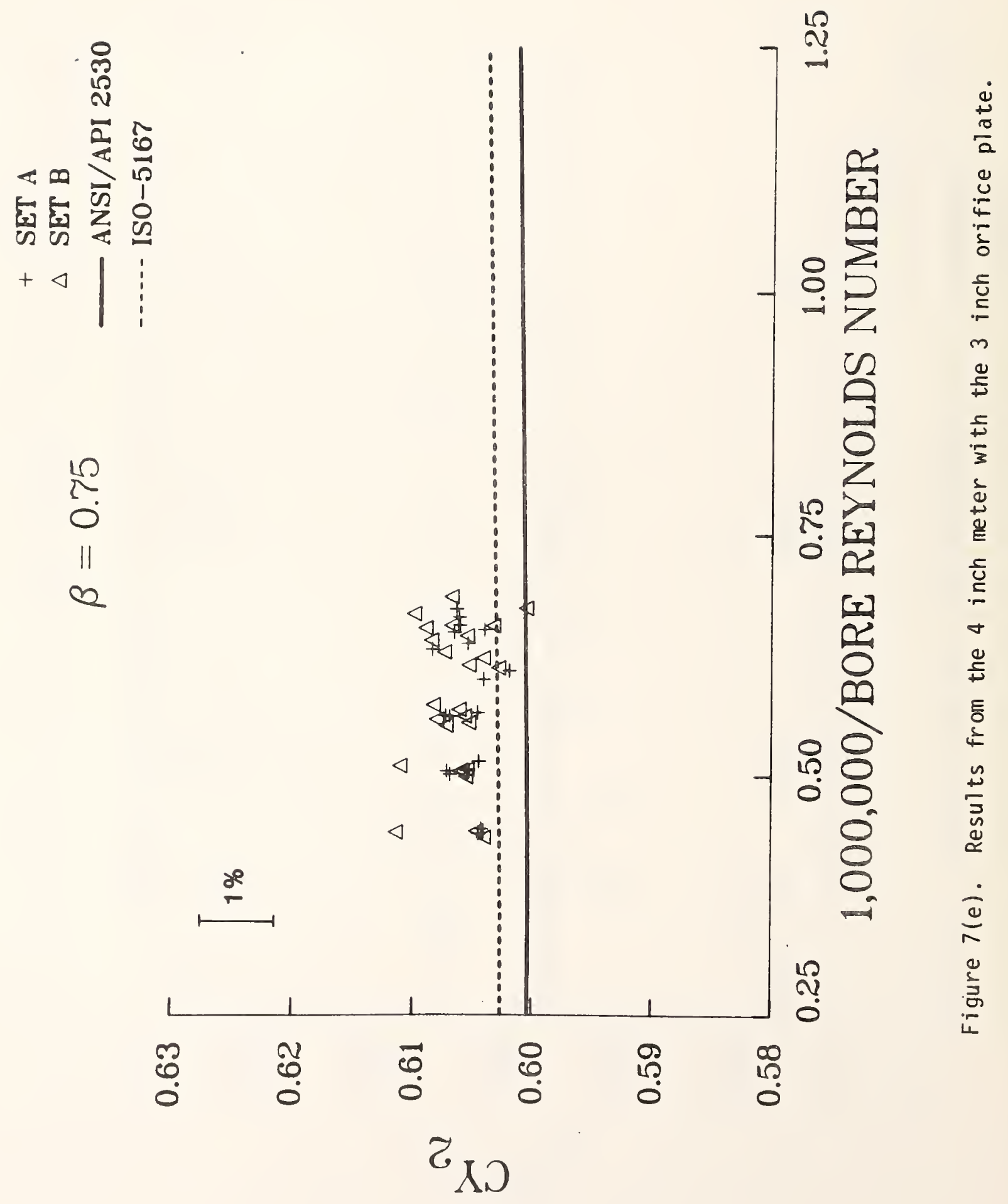




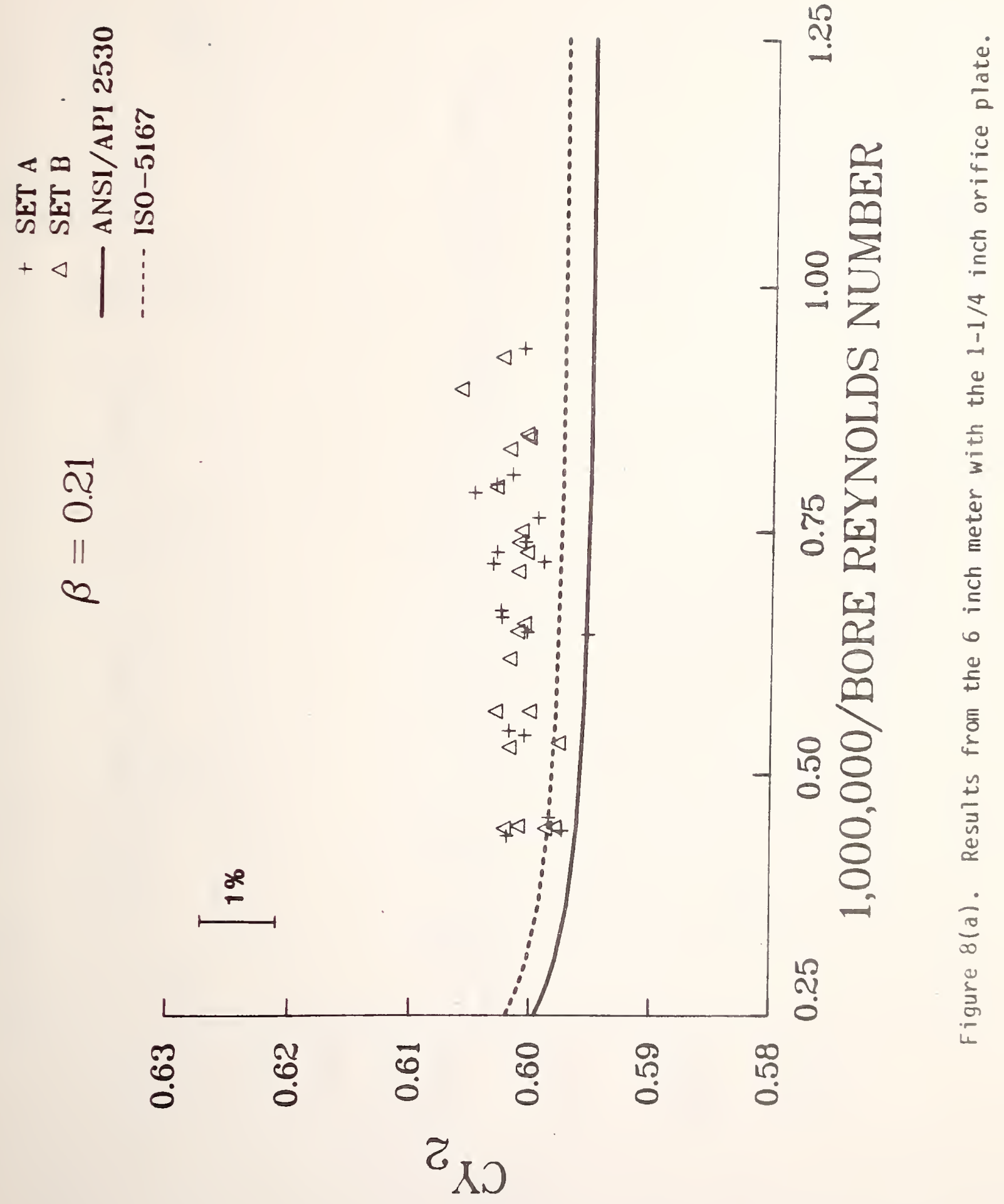



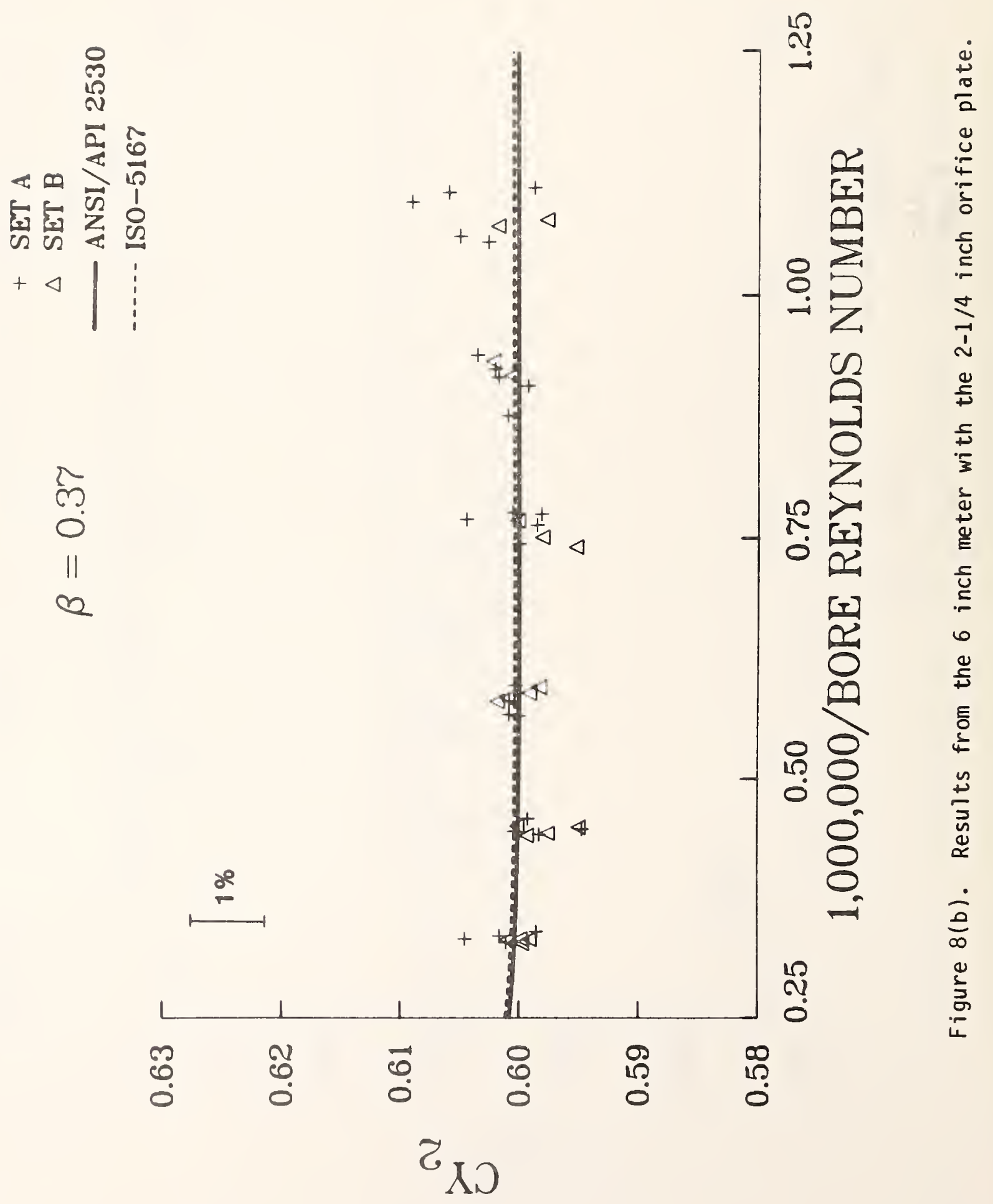


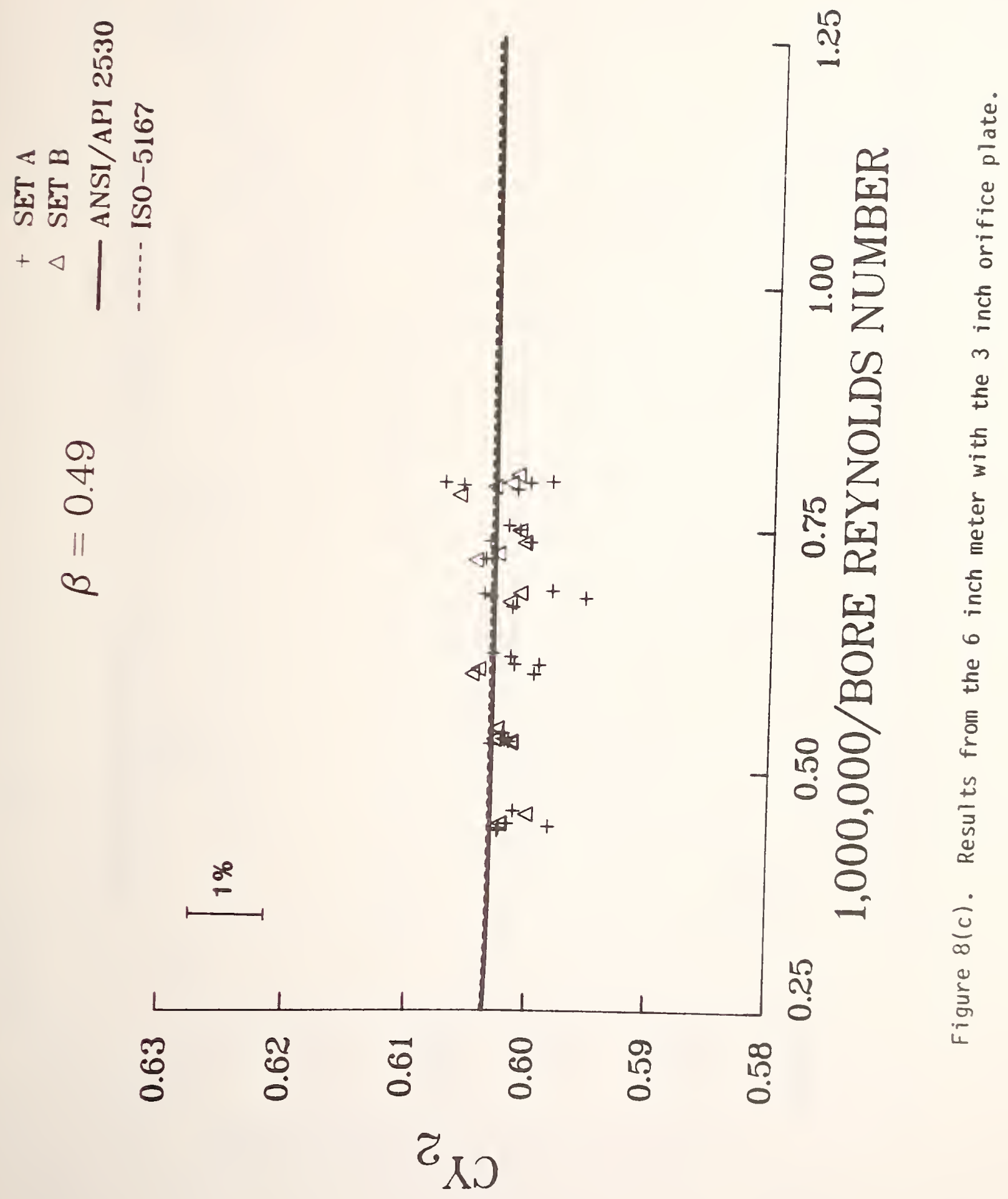




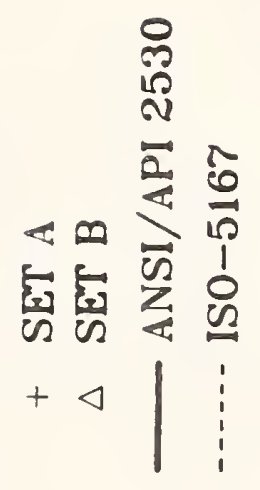

$\infty$
0
0

@

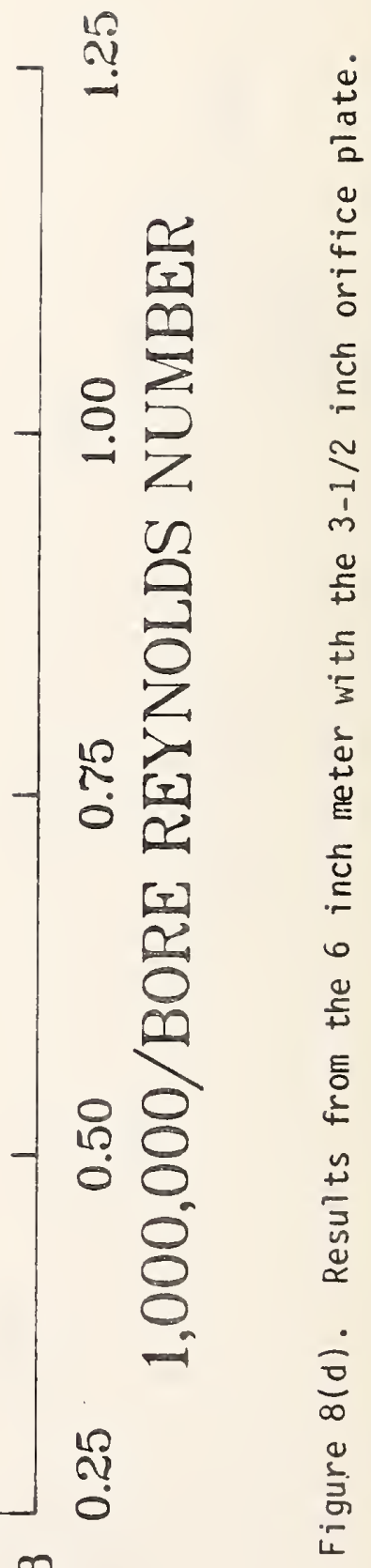

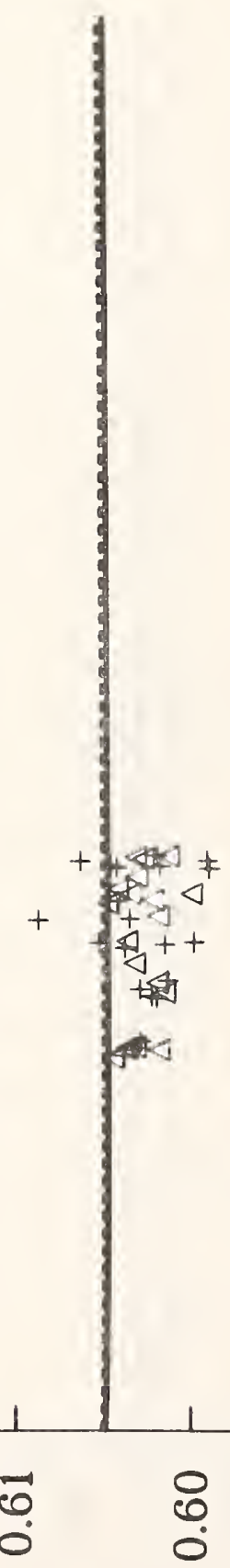

${ }^{2}$ XN 


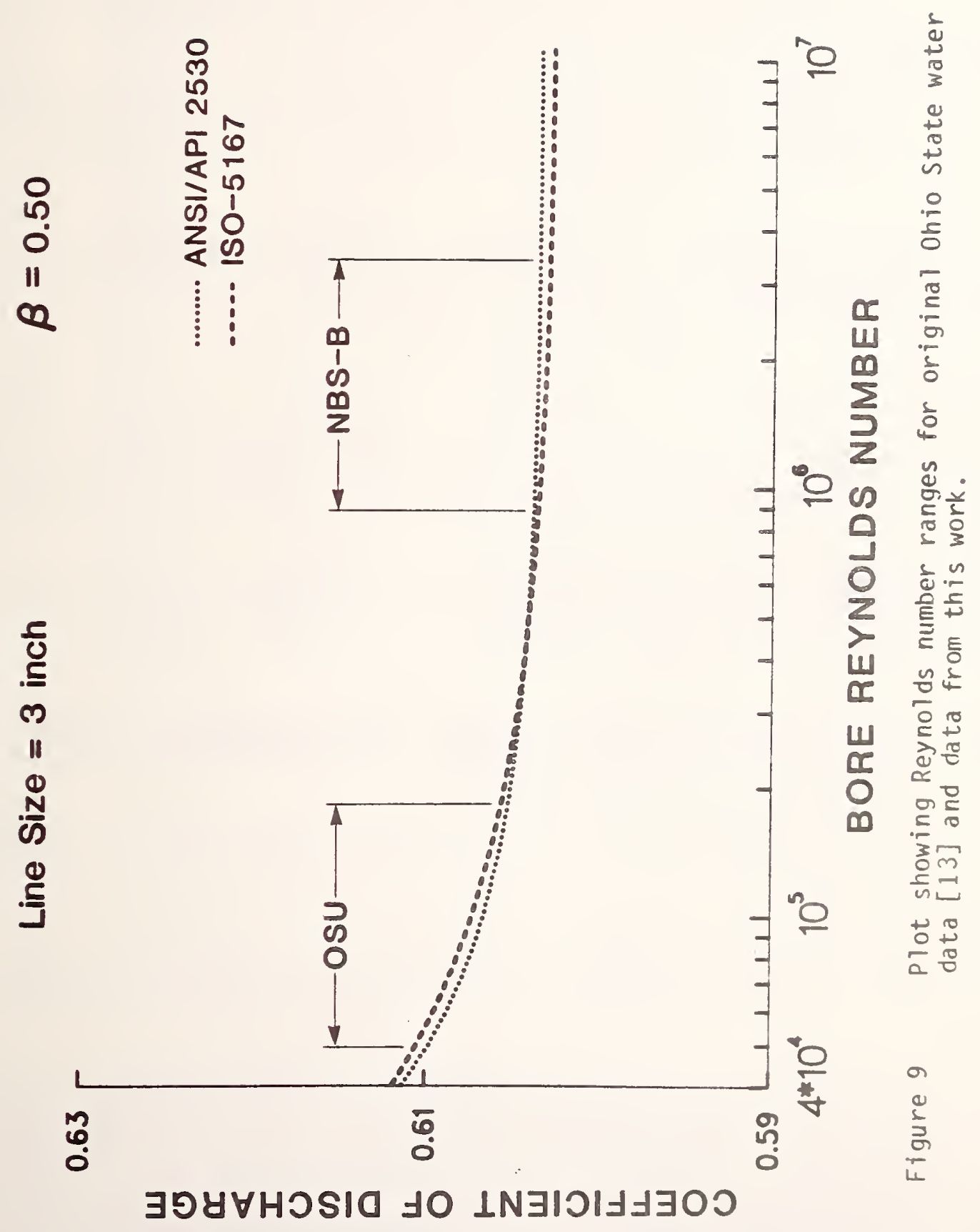


Table 2(a). Test Meter Identification and Physical Measurements Nominal 2 Inch AGA/API Orifice Meter (SI Units).

$\begin{array}{ccccc}\text { Test } & \text { Test } & \text { Run } & \text { Orifice } & \text { Beta } \\ \text { Identification } & \text { Configuration } & \text { Diameter } & \text { Diameter } & \text { Ratio } \\ & (\mathrm{cm}) & (\mathrm{cm}) & \end{array}$

$\begin{array}{lllll}\mathrm{BFEl}-4 \mathrm{~A}-072981-[9] & 1981-\mathrm{C} & 5.2520 & 2.8555 & 0.5437 \\ \mathrm{BFEl}-4 \mathrm{~A}-050582-[9] & 1981-\mathrm{D} & 5.2520 & 2.8555 & 0.5437 \\ \mathrm{BFE} 1-4 \mathrm{~B}-050682-[8] & 1981-\mathrm{D} & 5.2520 & 2.8565 & 0.5439 \\ \mathrm{BFE} 2-4 \mathrm{~A}-011282-[5] & 1981-\mathrm{D} & 5.2598 & 2.8555 & 0.5429 \\ \mathrm{BFE} 2-4 \mathrm{~A}-011482-[1] & 1981-\mathrm{D} & 5.2598 & 2.8555 & 0.5429 \\ & & & & \\ \mathrm{BFE} 2-4 \mathrm{~A}-011582-[9] & 1981-\mathrm{D} & 5.2598 & 2.8555 & 0.5429 \\ \mathrm{BFE}-4 \mathrm{~B}-011482-[8] & 1981-\mathrm{D} & 5.2598 & 2.8565 & 0.5431 \\ \mathrm{BFEl}-5 \mathrm{~A}-073081-[9] & 1981-\mathrm{C} & 5.2520 & 3.4917 & 0.6648 \\ \mathrm{BFEl}-5 \mathrm{~A}-050682-[9] & 1981-\mathrm{D} & 5.2520 & 3.4917 & 0.6648 \\ \mathrm{BFEl}-5 \mathrm{~B}-111881-[9] & 1981-\mathrm{D} & 5.2520 & 3.4915 & 0.6648 \\ & & & & \\ \mathrm{BFEl}-5 \mathrm{~B}-050582-[9] & 1981-\mathrm{D} & 5.2520 & 3.4915 & 0.6648 \\ \mathrm{BFE} 2-5 \mathrm{~A}-011382-[9] & 1981-\mathrm{D} & 5.2598 & 3.4917 & 0.6639 \\ \mathrm{BFE} 2-5 \mathrm{~B}-011382-[9] & 1981-\mathrm{D} & 5.2598 & 3.4915 & 0.6638 \\ \mathrm{BFE} 2-5 \mathrm{~B}-011582-[9] & 1981-\mathrm{D} & 5.2598 & 3.4915 & 0.6638 \\ \mathrm{BFEl}-6 \mathrm{~A}-072981-[8] & 1981-\mathrm{C} & 5.2520 & 3.8108 & 0.7256 \\ \mathrm{BFEl}-6 \mathrm{~A}-073181-[9] & 1981-\mathrm{C} & 5.2520 & 3.8108 & 0.7256 \\ \mathrm{BFEl}-6 \mathrm{~A}-111881-[7] & 1981-\mathrm{D} & 5.2520 & 3.8108 & 0.7256 \\ \mathrm{BFEl}-6 \mathrm{~B}-073081-[9] & 1981-\mathrm{C} & 5.2520 & 3.8105 & 0.7255 \\ \mathrm{BFEl}-6 \mathrm{~B}-073181-[9] & 1981-\mathrm{C} & 5.2520 & 3.8105 & 0.7255\end{array}$

Note: Test Identification bracket gives number of tests run with this geometry. A total of 154 tests were run on the nominal 2 inch run size. 
ஜேீீேே:

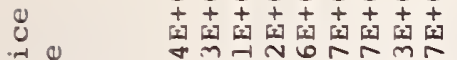

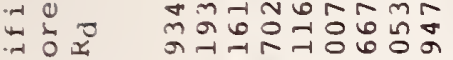
告 $\neg-N \rightarrow N-1-1$

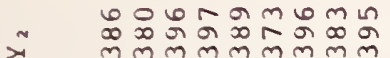
* - $\dot{0} \dot{0} \dot{0} \dot{0} \dot{0} \dot{0}$

Uน ฟิल

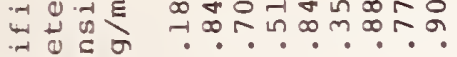

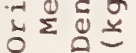

mariraráa

$\ln 300$

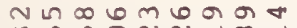
त $\sum E \simeq \pm$ i

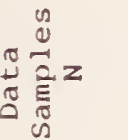

N-1の

न्न

- $\dot{n}^{m}$

4in is 0

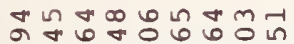
एँ

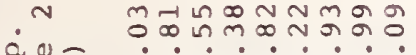
E ¿

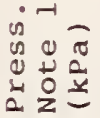

a mom in- $\dot{0} \dot{0} \dot{\sim} \dot{\sim}$

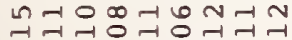
崖企

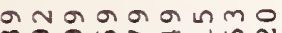

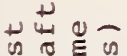
C) है1
0
0
0

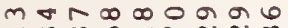
$\rightarrow m \infty$ I $\rightarrow N$ N ம 0 in in in 0

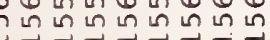

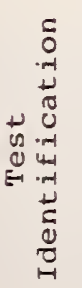

4n $\begin{array}{ccccccccc}1 & 1 & 1 & 1 & 1 & 1 & 1 & 1 & 1 \\ -1 & -1 & -1 & -1 & -1 & -1 & -1 & -1 & -1\end{array}$ $\infty \infty \infty \infty \infty \infty \infty \infty \infty \infty$ ก nnNNhNnN 000000000

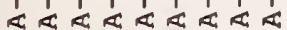

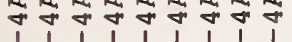

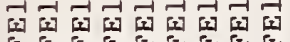

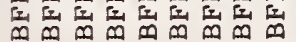

○ $+t+t+t+$

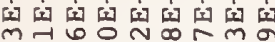
N $\cap+\infty$ N $N$ in -10 $m+\omega$ o $N$ - 00 o N $\dot{v} \dot{i} \dot{i} \dot{i} \dot{i}$

แกㅇㅇำMN Mำ ช 60606606 ல் $\dot{0} \dot{0} \dot{0} \dot{0}$

क ammarrm

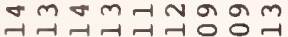

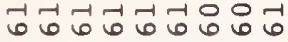
ம்

$m+\infty N-10 \infty-1$

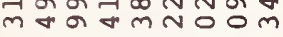
$\dot{\infty} \dot{\infty} \dot{\infty} \dot{\infty} \dot{\infty} \dot{\infty} \dot{\infty}$

ก ज̂ञ $\dot{\infty} \dot{\infty} \dot{\infty} \dot{\infty} \dot{\infty} \dot{0}$

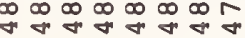

$+\pi \omega m$ in मmनr $\infty \infty_{\infty \rightarrow \infty}^{\infty}$

m $\infty 0 \pi N N$ N -1 ๑ 60606 0000000

$\rightarrow-10$ 06 ல் 000

mm nO

$\exists$ 은

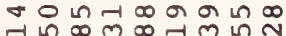
o in $\infty$ in ப் $0 \dot{0} 0 \dot{0}$

$m a+m m \infty \infty 0 m$

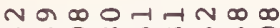
$\forall \infty a \infty$ in $m$ in a

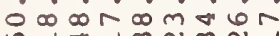

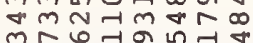
जก ○ं000000

षดก

$N m$ N

$\infty$ ก 1 a $\infty$ N ○ Nक का NRM

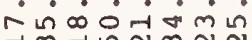

$\leftarrow \nleftarrow$ mog i. Ni $\infty$

n mn m แn $\infty \pi$ 00000

N in 0

บำก - 0ं $\dot{0}$ i $\dot{0}$ i

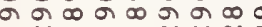
ก บ N N บ N

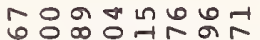

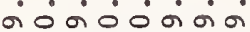
$\infty$ o $\infty$ न $\sigma \infty^{\infty} \infty$

แn $\mathrm{N}$ o 0 แn í $\dot{0} \dot{0} \dot{m} \dot{\sigma} \dot{m}$ m

$\neg N \infty m m N \infty N$

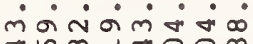

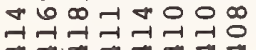

윙 i் का $\sigma$ \%

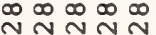

$\infty \neg \sim 6 \mapsto$ i $00 \div 0$

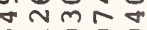
$0 \rightarrow 00$

$0_{0}^{m}$

anra n⿺辶一 - . - i. - $N$ N ar $\sim$ N

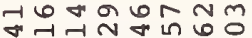

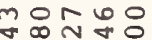
$\infty \pi n m$ ம் m N N $\mathrm{m}$ m $\mathrm{N} N \mathrm{~N}$ Nํㄷํ m

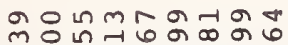
- $\dot{\sim} \dot{\sim} \dot{\sim} \dot{\sim} \dot{\sim}$

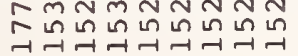

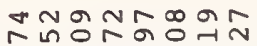

늠 ?.? $+N \rightarrow 6 r$ ก न ब r $\infty<r \infty \infty \infty$

-

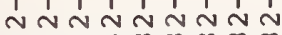

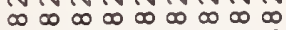

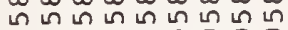
잉ㅇㅇㅇㅇㅇㅇㅇ นn

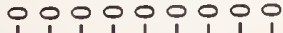
$\begin{array}{lllllllll}1 & 1 & 1 & 1 & 1 & 1 & 1 & 1 & 1\end{array}$

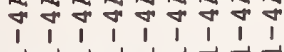

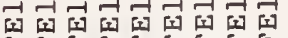

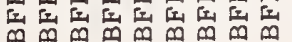

-NM

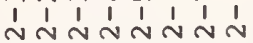
$\infty \infty \infty \infty \infty \infty$ ๒ nn

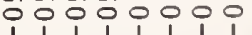
$\begin{array}{llllllll}1 & 1 & 1 & 1 & 1 & 1 & 1 & 1 \\ 0 & 0 & 0 & 0 & 0 & 0 & 0 & 0\end{array}$

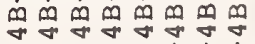

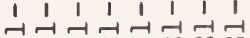

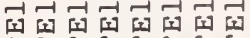

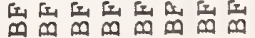

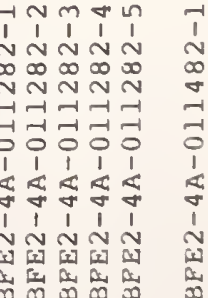




\section{U

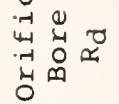

$\stackrel{2}{2}$

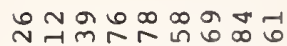

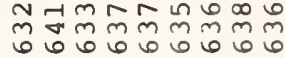
○ं

nก⿻m乚n

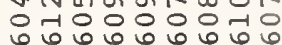
ல0்00்0ல0

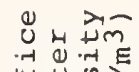
出

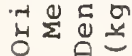

ปี

雨是

ठ $\sigma^{2}$

¿

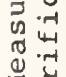

造

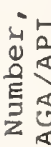

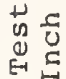

$\therefore \sim$

苗

$a^{2}$

는

$\stackrel{0}{-1}_{-1}^{\infty}$

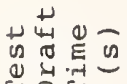

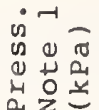

$\sum_{\substack{0 \\ 0}}^{\infty}$

m-

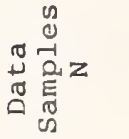

ํํำกㅇำ

- in is $^{m}=$

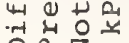

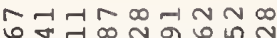

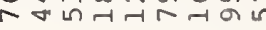
o 0 -

ㅇำสำ

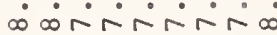

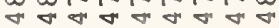

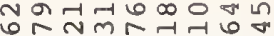

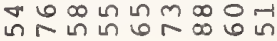
○ं0ல0ல000

는웅

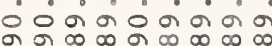

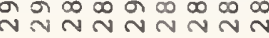

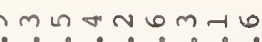
ᄀᄀ

mூom $\dot{0} \dot{0} \dot{0} \sim \dot{\infty} \dot{\infty}$

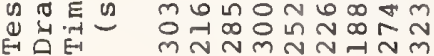
ט 0.

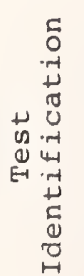

HNm n งั่ง

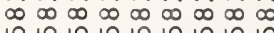
-1 -1-1-1 - $-1-1$

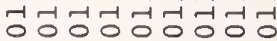

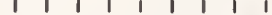

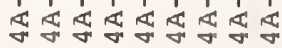

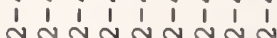

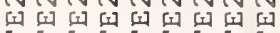

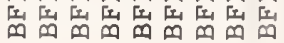
ที่ ○ं0ல0000

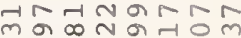
का

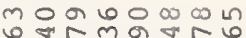
बना $\therefore \infty-i N m$ 传

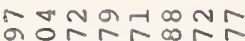
$\therefore-\dot{0} 0 \circ 0 \dot{0}$

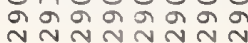

டீலீ:

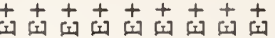
ON N Nูํํำ

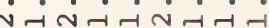

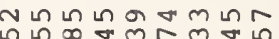

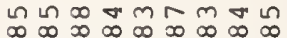
$\begin{array}{lllllllll}\infty & \infty & 0 & \infty & \infty & 0 & \infty & \infty & \infty \\ \omega & 0 & 0 & 0 & \ddots & 0 & 0 & 0 & 0\end{array}$ ○ं0ல00000

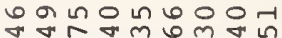

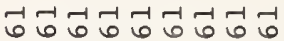
ல் ப்

กิกตต

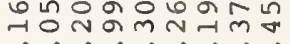

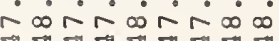

月, ก m $\infty$ m $\infty$ -

๒

की ๙ ம்

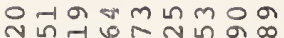
- $\cdot$. . $\cdot$ ने न न न न न न न न ข $N$ N N

๑ $N m \infty \pi m-$ ن

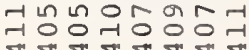

o onm-nR - $\dot{0} \dot{\sim} \dot{0} \dot{0} \dot{0} \dot{0}$ ๒กำ

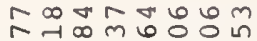
$\dot{\sim} \dot{\infty} \dot{\infty} \dot{0} \dot{0} \dot{0}$ வ m N $\sim \mathcal{N} \sim \sim$

$0-1 m-10$ เ $N$ N $\infty$ ก $661066 \omega$

- $14 \pi \operatorname{1n} 6 \pi \infty$

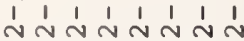
$\infty \infty \infty \infty \infty \infty \infty$ 1 덩더엉어엉엉

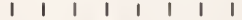
m

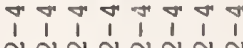

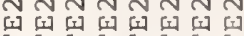

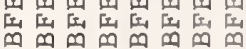

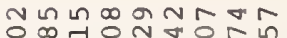

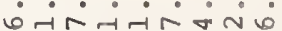
NAก ก

$m \sim \infty \circ m m m \infty \infty$

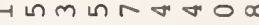
nNNNNNNm-1

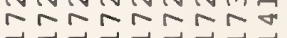

-1 2 แ $\begin{array}{ccccccccc}1 & 1 & 1 & 1 & 1 & 1 & 1 & 1 & 1 \\ -1 & -1 & -1 & -1 & -1 & -1 & -1 & -1 & -1\end{array}$

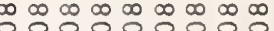
m $m$ m $m$ m ranaranar

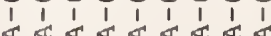

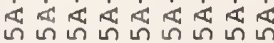

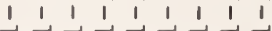

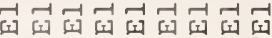

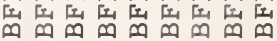

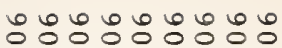

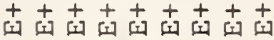
$\infty$ नू $\infty$ m न -

ช न శ) 00000000

ㄴำ 에 ข 웅ㅇㅇㅇㅇ

ம ก สำ

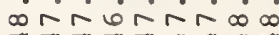

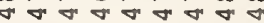

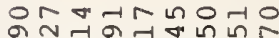
न $N m \infty 0 \sim 6 N$ ทी ○் $\dot{0} \dot{0} \dot{0}-\dot{1} \dot{0}$

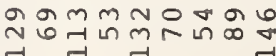

-10人。m

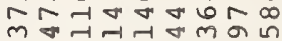

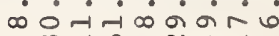

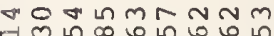
- $\dot{0} \dot{0} \dot{0} \dot{0} \dot{0}$ $\infty \infty \infty \infty \infty \infty \infty \infty \infty$

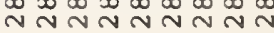

$\operatorname{manh}$ वं $\dot{0} \dot{\infty} \dot{-1} \dot{0} \dot{-r} \dot{m}$

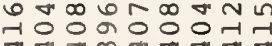

অ aं $\dot{0} \dot{\sigma} \dot{\sim} \dot{0} \dot{\sim}$ a กิน

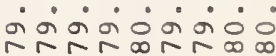

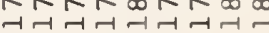

- 1 m กับ $\infty \infty \infty \infty \infty \infty \infty \infty \infty$ ๒ เn乚

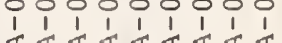
约结出出出出出

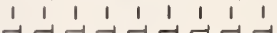

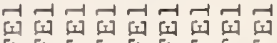

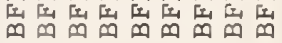




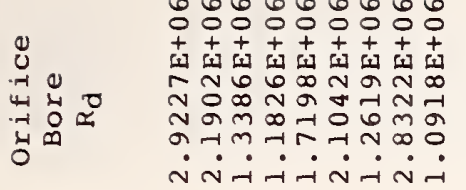

$\forall m \varphi \infty \pi \infty a 6$ $\infty \infty_{\infty} \infty \infty_{\infty} \omega_{\infty} \infty \infty_{\infty} \infty$

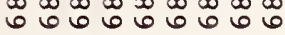
نं $\dot{0} 0 \dot{0} \dot{0} \dot{0} 0$

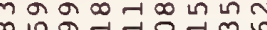
i็

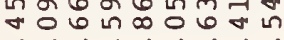
-i $\dot{0} \dot{0} \dot{-10}-\dot{0}$

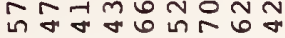

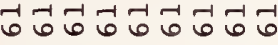
000000000

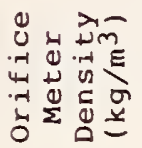

กNนM

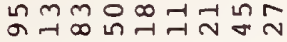
ن $\forall \theta$

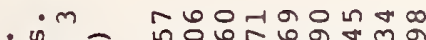
4 थ

出占艺 句台呈己

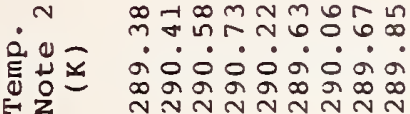

ir ranarin un ปึ

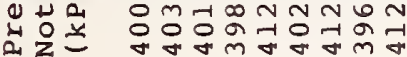
$a^{2}$ ก

(1) म न

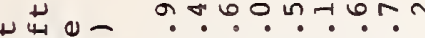
का

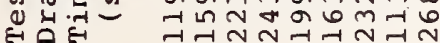

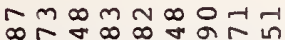
$\dot{\sim} \dot{0} \dot{m}-\dot{-} \dot{0} \dot{0}$

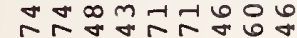

-1 $⿻$ आ

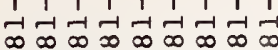

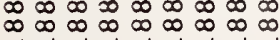
$\rightarrow-1 \rightarrow-1-1 \rightarrow-1 \rightarrow-1,-1$ ન્નコનન્નનન્ન $\begin{array}{llllllllllll}1 & 1 & 1 & 1 & 1 & 1 & 1\end{array}$ 品品品品品 $1,1,1,1,1$

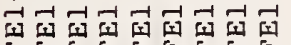

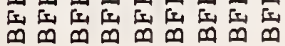

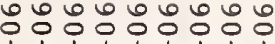
$+t+t+t+t+$

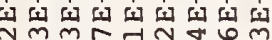
न 6 m 이요 त बूल arm $\mathrm{m} \pi-\mathrm{r}^{\circ}$ $\dot{\sim} \dot{\sim}-\dot{\sim} \dot{\sim} \dot{\sim} \dot{\sim}$

ํํำㄴำ กิ $\infty \infty \infty \infty \infty \infty \infty \infty$ : ? : -00000000

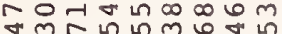

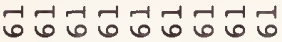
○ं 0 0 0000

mmo6m $m$ N ติ ińsirrón

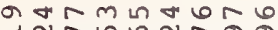
ใำ

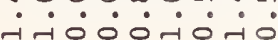

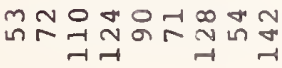

ก

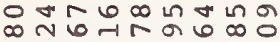

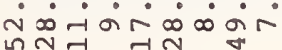

* - $\dot{0} \dot{0} \dot{0} \sigma^{\circ}$

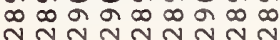

o om no a - $\dot{\sim} \dot{\sim} \dot{\sigma} \dot{\sigma} \dot{\sigma} \dot{\infty}$

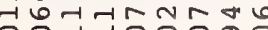

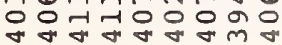

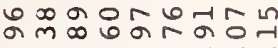
NRि-10ं

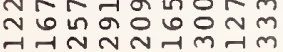

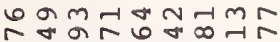
ட் कबका कून

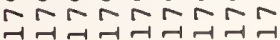

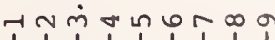
ง่ง ำ $\infty \infty \infty \infty \infty \infty \infty \infty \infty$

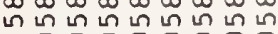
응ㅇㅇㅇㅇㅇㅇㅇㅇㅇㅇ เ ็ㅇํㅇㅇㅇㅇㅇㅇ 1
1

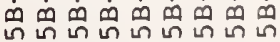
$\begin{array}{llllll}1 & 1 & 1 & 1 & 1 & 1\end{array}$

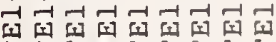

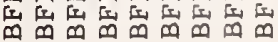

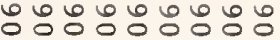
$++++++++++$

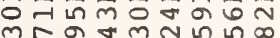
mก̃ ป ำ

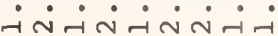

$\infty \infty \infty \infty$ a $n N m$ $\infty \sim \infty \infty \infty \pi \infty \infty$

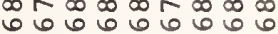
○0000000

ค) $m-1-1 \infty 6-1 \sigma$

ㅇำतสด न

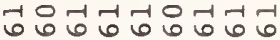
○ं $0 \dot{0} 0 \dot{0} 0$

NaOnharan manargun mกन

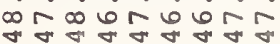

$\infty$ ก \& म

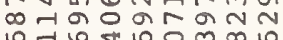

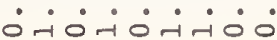

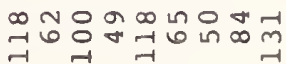

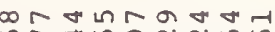
mกホ์ $\infty \circ-\dot{0} \infty \dot{0} 0$

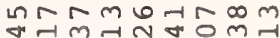
○ं0் 0000.

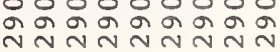

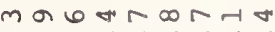
ن $1 \dot{0} \dot{0} \dot{0} \dot{0} \dot{0}$

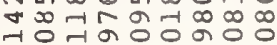

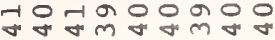

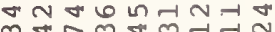
- $\dot{0} \cdot \dot{0} \cdot$ แnm ง

$m \pi n+m a m n \infty$ 大 0 en Ln พั่ง $\dot{\sim} \dot{\sim}-\dot{v}$ ชิ 60060060

-

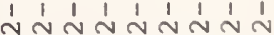

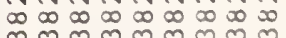
mलm mलm

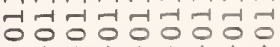

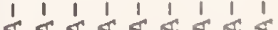

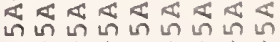

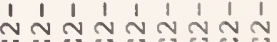

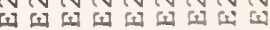

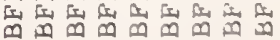

○ீலீல்: $++t+++$

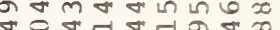

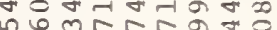
กำก nก

* $m-\infty \cup \infty N m$

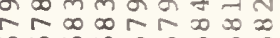

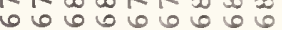
000000000

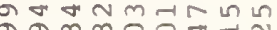
grmmo

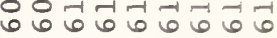
000000000

Nㅡㅇำกำ

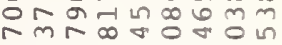

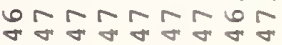

ตกคํำน N $\infty 0^{\infty} \infty \mathrm{m}^{\infty}$ \% 0 U -

भ่

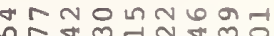

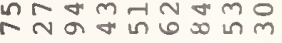
Ho

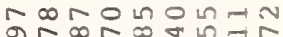
00000000

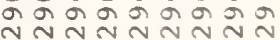

a mal m onía

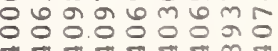

म 40 नI

N $\dot{m} \dot{0} \dot{0} \dot{\sim} \dot{\sim} \dot{\sim}$ o

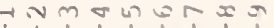

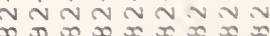

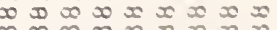

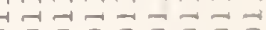
- 00000000

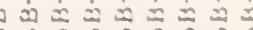

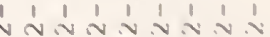

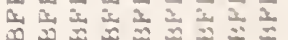


ஜ๐๐ㅇㅇ

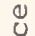

$\begin{array}{lll}-1 & 0 \\ -4 & 4 & 0 \\ -1 & 0 & 0 \\ 0 & 0\end{array}$

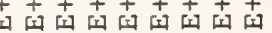

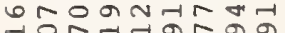
음 는

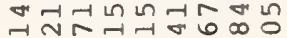
$\dot{\sim} \ddot{-} \dot{\sim} \dot{-} \dot{\sim} \dot{\sim}$

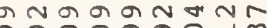
न क०० का० ना 6 6 0606060 - $\dot{0} \dot{0} \dot{0} \dot{0} \dot{0}$

Uै सेल - 1 (1) $\Rightarrow$ ह $4+$ on -

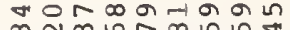
\% กิ $\therefore \infty \dot{\infty} \dot{\infty} \dot{\infty} \dot{\infty} \dot{\infty}$ mกN N $N$ N

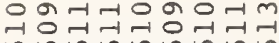
? ? 000000000

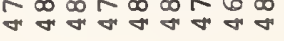

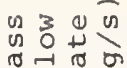

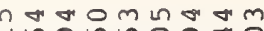

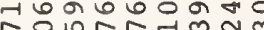

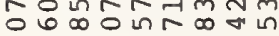
-10்-10்0்

范

बำ

- is $\dot{n}^{m} \approx$

出 0100

邱出

$\pi \infty 6 N \approx m \pi N N$

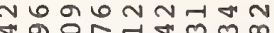
$\dot{v} \infty \dot{\sim} \infty \dot{N} \dot{\sim} \dot{\sim}$ ก ก

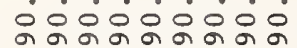

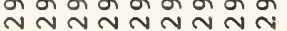

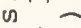

(1) 15

$m \infty$ 几 - $\infty$ a w 0 Z

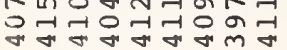

이는

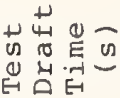
.

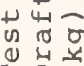

ดก $N$ N

เก $N$.

(4) 넌

$m \sim \dot{m} \dot{m} \dot{m} \dot{m}$

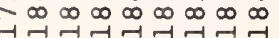

- 1 m

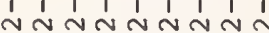
$\infty \infty \infty \infty \infty \infty \infty \infty \infty$ in in in in in in in in ન્નનનન ન્ન ન્ન 000000000

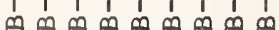

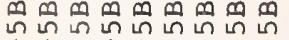
ง N

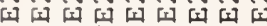

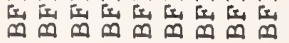

అீ人

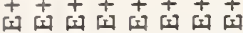

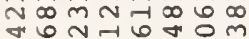

ป

N

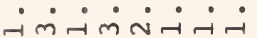

6 न ब क जN Nm $\mathrm{N}$ N $\mathrm{N}$ in NNNNNTNN - $\dot{0} \dot{0} \dot{0} \dot{0} \dot{0}$

mกษ

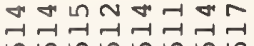
:

hom $N$ in on $N$

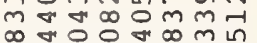

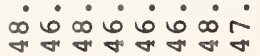

- 0 क क क

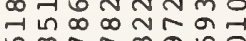

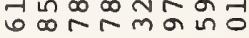
ம் $\dot{0} \dot{-1} \dot{0} \dot{0}$

の윰ำ

$m \infty-m+\omega \infty 6$ ด ก $N m 06 \infty$ ? ம்

mमก $\sim \dot{\sim} \dot{\sim}-\dot{\sigma}-\dot{\sigma}-$

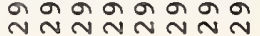

O ONMNNND -نि-ن் $\dot{0} \dot{0}$ $\sim \infty$ in $\infty m \omega$

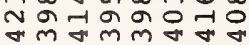

Noเ ด. नmavoro न न क ल न

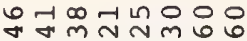
ن NNNNNNNN Nan

-1 Nm

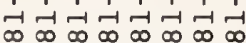
न न न न न क न न NNNNNNNN r r N N N N N N i i i i i i i i 芯芯 $\begin{array}{llllllll}1 & 1 & 1 & 1 & 1 & 1 & 1 & 1\end{array}$

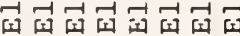

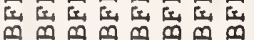

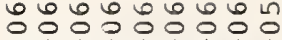

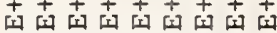
स ब కస

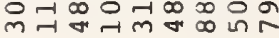
$\dot{m} \dot{\sim} \dot{-i} \dot{-} \dot{\sim} \dot{\sigma}$

$\circ \infty$ ค

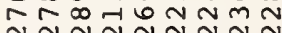

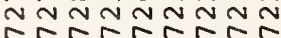
- $0 \dot{0} \dot{0} \dot{0} 0 \dot{0}$

HกN $\infty \infty \pi m \pi m$ m $6 \sigma 6 \sigma 6 \sigma 66$ ல் $\dot{0} \dot{0} \dot{0} \dot{0} 0$

on $m \in$ o

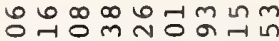
ம் $\dot{0} \dot{0} \dot{\infty} \dot{N} \infty$

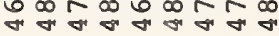

MN $\infty N \omega \pi$ స్그눈 $\infty 6 \mathrm{~m}$ o $\infty 0 \mathrm{~m}$ in -ن்- $\dot{0} \dot{\circ} \dot{\circ} \dot{-1}$

NmLnnN $n$ n

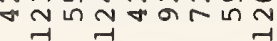

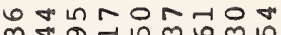
ข - $\dot{0} \dot{0} \dot{0} \dot{0} \dot{0} \dot{0}$ in $N$ ก $N$

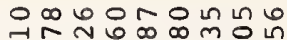
Hก - $\dot{0} \dot{0} \dot{0} \dot{0} \dot{0}$

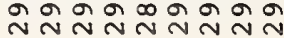

nกL $\dot{\sigma} \dot{\infty} \dot{\infty} \dot{m} \dot{0} \dot{\sim}-$ $m \sim N+n=m$ in

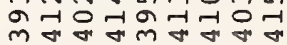

rma이의 $0.5 \pi$ - 0 क 0 i

न

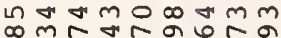

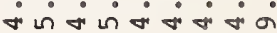
hrtrktrkg

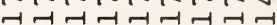

- $m m$ n

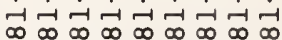

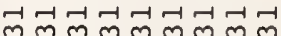
rantranta

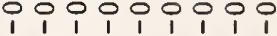

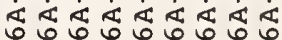
$\begin{array}{lllllll}1 & 1 & 1 & 1 & 1 & 1 & 1\end{array}$

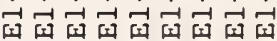

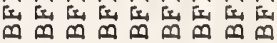

๕ะะะะ๐

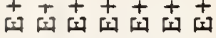
NOH 0 แ *

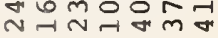
$\dot{m} \dot{m} \dot{\sim} \dot{\sim} \dot{\sim}$

ब가의 NN N N N hrnhrn Oீं0000

a $\infty$ IN 26 กี . -00000

$m m \infty m i n$ の iñ

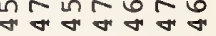

$N$ o 0 - $N \infty \infty$ $m \infty$ 네 $n-1, \infty$ ก -i $\dot{0} \dot{0} \dot{0} \dot{0}$

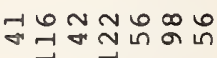

a N 2 a 00 แก 吕

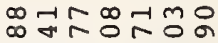
- $-\dot{0} \dot{0} \dot{0} \dot{0}$ กำกำกั

NTO In 6

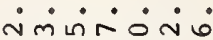
๘ี

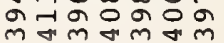

$\infty r a \infty 60 \Omega$ NTr In $\ln \infty$ o 0 - nn- 0 N $\sim m m m$

Nob ํNNNNN ann

T⿻ 거 거다 거마 $\begin{array}{lllllll}\infty & \infty & \infty & \infty & \infty & \infty & \infty\end{array}$

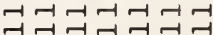
거규규 ๔ $\begin{array}{lllllll}1 & 1 & 1 & 1 & 1 & 1\end{array}$

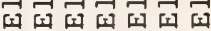

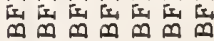




\section{(1) 南南南南南南南

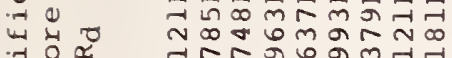 - i $\dot{\sim} \dot{\sim} \dot{-m} \dot{m} \dot{m}$}

ลㅇำ $N \sim \pi N N$ N $N$ NRTNRNAN

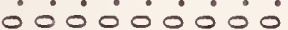

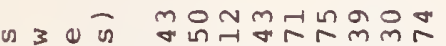

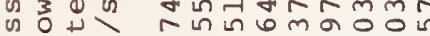

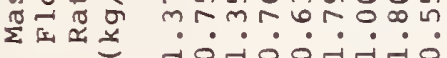
党管

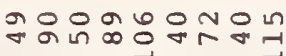
- $\dot{n}^{m}-$

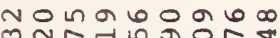

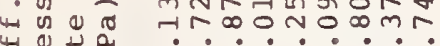
$\rightarrow$

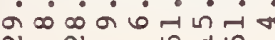

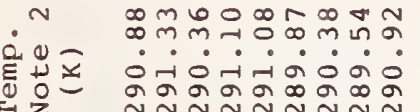

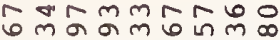
$\therefore \dot{0} \dot{0} 00$ กำ

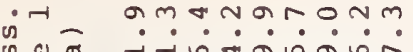

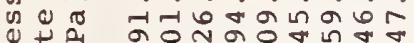

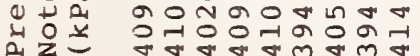

क-rmbro nm லம்பீ்

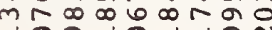

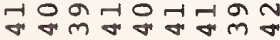

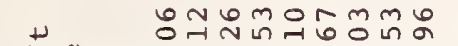
पु

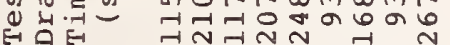

T约。 -

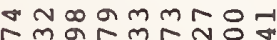

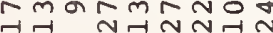

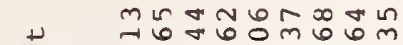
$4 \psi$ ज व का $\infty \infty \infty \infty \infty \infty \infty \circ$

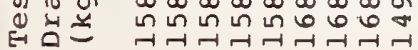

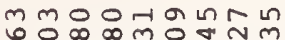
- $\dot{0} \dot{0} \dot{0} \dot{0}$

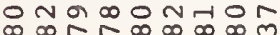

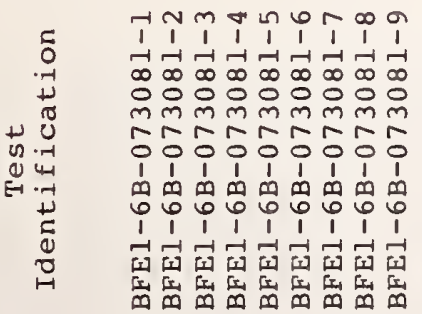

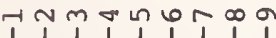
$\rightarrow-7-1-1$ $\rightarrow-1-1-1-1-1$

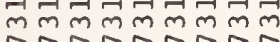

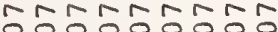
1 1 111111

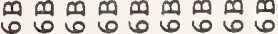
1 111111

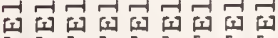

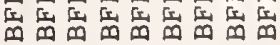

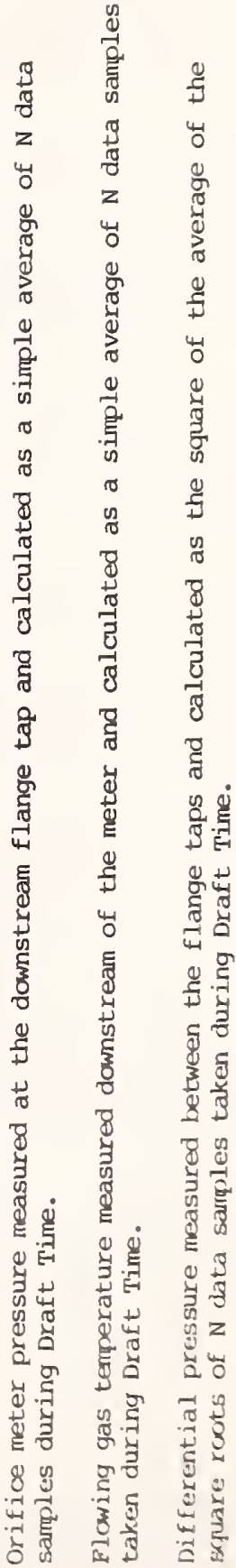

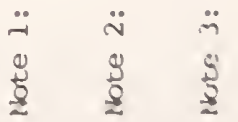


Table 3(a). Test Meter Identification and Physical Measurements Nominal 3 Inch AGA/API Orifice Meter (SI Units).

Test

Identification

$\mathrm{BFE} 3-2 \mathrm{~A}-011982-[8]$
$\mathrm{BFE} 3-2 \mathrm{~B}-012182-[9]$
$\mathrm{BFE} 4-2 \mathrm{~A}-070782-[6]$
$\mathrm{BFE} 4-2 \mathrm{~B}-070782-[4]$
$\mathrm{BFE} 4-2 \mathrm{~B}-070982-[9]$
$\mathrm{BFE} 3-3 \mathrm{~B}-012282-[9]$
$\mathrm{BFE} 4-3 \mathrm{~A}-061981-[9]$
$\mathrm{BFE} 4-3 \mathrm{~A}-072381-[9]$
$\mathrm{BFE} 4-3 \mathrm{~B}-061881-[9]$
$\mathrm{BFE} 4-3 \mathrm{~B}-072281-[9]$
$\mathrm{BFE} 3-4 \mathrm{~B}-011982-[9]$
$\mathrm{BFE} 3-4 \mathrm{~B}-012282-[9]$
$\mathrm{BFE} 4-4 \mathrm{~A}-061981-[9]$
$\mathrm{BFE} 4-4 \mathrm{~A}-072181-[9]$
$\mathrm{BFE} 4-4 \mathrm{~B}-070282-[9]$
$\mathrm{BFE} 3-5 \mathrm{~A}-012082-[8]$
$\mathrm{BFE} 3-5 \mathrm{~B}-012082-[9]$
$\mathrm{BFE} 4-5 \mathrm{~A}-070282-[9]$
$\mathrm{BFE} 4-5 \mathrm{~B}-070882-[10]$
$\mathrm{BFE} 3-6 \mathrm{~B}-012182-[8]$
$\mathrm{BFE} 4-6 \mathrm{~A}-061781-[9]$
$\mathrm{BFE} 4-6 \mathrm{~A}-072281-[9]$
$\mathrm{BFE} 4-6 \mathrm{~B}-070882-[9]$

Test Configuration

$1982-\mathrm{A}$
$1982-\mathrm{A}$
$1982-\mathrm{A}$
$1982-\mathrm{A}$
$1982-\mathrm{A}$
$1982-\mathrm{A}$
$1981-\mathrm{B}$
$1981-\mathrm{B}$
$1981-\mathrm{B}$
$1981-\mathrm{B}$
$1982-\mathrm{A}$
$1982-\mathrm{A}$
$1981-\mathrm{B}$
$1981-\mathrm{B}$
$1982-\mathrm{A}$
$1982-\mathrm{A}$
$1982-\mathrm{A}$
$1982-\mathrm{A}$
$1982-\mathrm{A}$
$1982-\mathrm{A}$
$1981-\mathrm{B}$
$1981-\mathrm{B}$
$1982-\mathrm{A}$

Run Diameter (cm)

7.7925
7.7925
7.7922
7.7922
7.7922

7.7925

7.7922

7.7922

7.7922

7.7922

7.7925

7.7925

7.7922

7.7922

7.7922

7.7925

7.7925

7.7922

7.7922

7.7925

7.7922

7.7922

7.7922
Orifice

Diameter (cm)

$\begin{array}{ll}2.8575 & 0.3667 \\ 2.8572 & 0.3667 \\ 2.8575 & 0.3667 \\ 2.8572 & 0.3667 \\ 2.8572 & 0.3667\end{array}$

3. 8092

3.8100

3.8100

3. 8092

3. 8092

0.4888

0.4890

0.4890

0.4889

0.4889

\subsection{5}

4. 4445

0.5704

4.4458

4.4458

4.4445

0.5704

0.5705

0.5705

0.5704

5.0815
5.0795

0.6521

5.0815

0.6519

0.6521

5.0795

5.7140

0.6519

0.7333

$\begin{array}{ll}5.7130 & 0.7332 \\ 5.7130 & 0.7332 \\ 5.7140 & 0.7333\end{array}$

Note: Test Identification bracket gives number of tests run with this geometry. A total of 197 tests were run on the nominal 3 inch run size. 


\section{$\int_{\substack{0 \\-1}}^{0} 0$}

年
ஜㅇ๐유

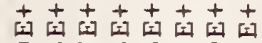
Oढि ำำกำ

inisi.

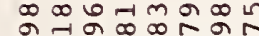

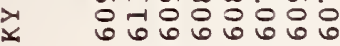 0ं0்000}

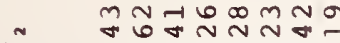

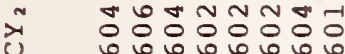
$\therefore 0.0000$

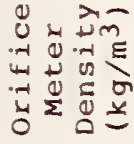

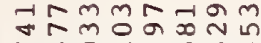
non

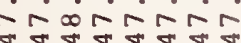

ด

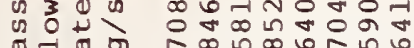

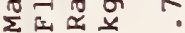
类 0000000

HMUNmก- $\infty$ $\exists \operatorname{mon} \underset{-1}{ } \underset{-1}{\infty}$

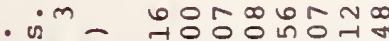

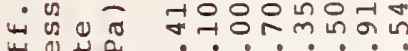
ou

$\frac{1}{\frac{1}{0}}$

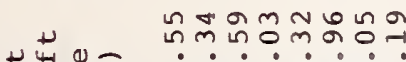
造

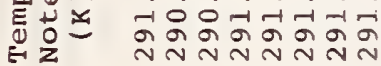

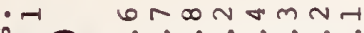
ज०

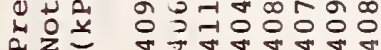

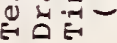

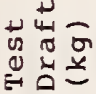

ํํำ

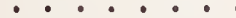
mмm mиㄴ

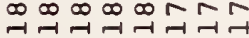

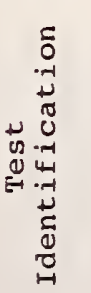

$\operatorname{1nman\operatorname {cos}}$

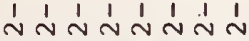
$\infty \infty \infty \infty \infty \infty \infty$

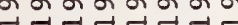

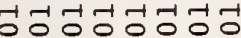
111111111

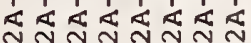

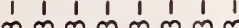

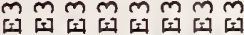

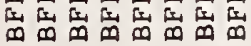

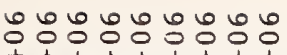
南卉南南南南南南南

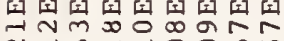

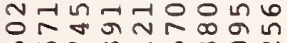

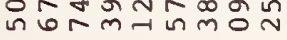

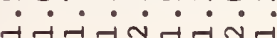

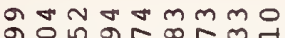

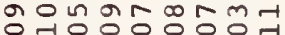
0600000006 00000000.

의 ำ

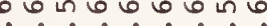
○ं0 00000

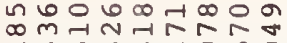
๓

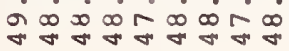

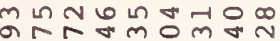

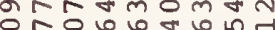

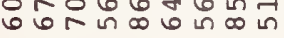
000000000

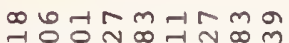
검ำ

ก ชิ

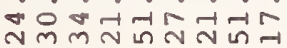

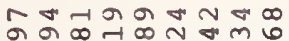
ம் 虽 $\stackrel{\infty}{\sim} \stackrel{\infty}{\sim} \stackrel{\infty}{\sim} \stackrel{\infty}{\sim} \stackrel{\infty}{\sim} \stackrel{\infty}{\sim} \stackrel{\infty}{\sim} \sim$

Ho ONN

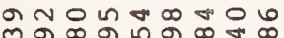

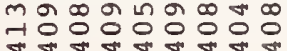

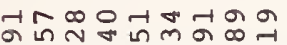
$\therefore \dot{0} \dot{0} \dot{0} \dot{0} \dot{0} \dot{0}$ 붕ํำ N N N $N$ N

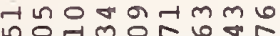
- $\dot{0} \dot{0} \dot{0} \dot{0} \dot{0}$ artrablo

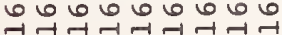

TNm

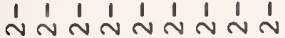

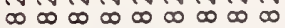

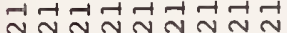

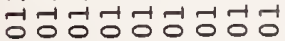
$\begin{array}{lllllll}1 & 1 & 1 & 1 & 1 & 1 & 1\end{array}$

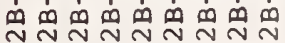

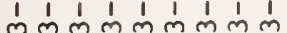

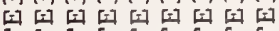

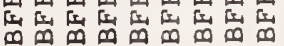

ํㅜㅇํㅇㅇㅠ 南南南南南 जo의

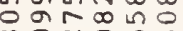

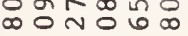

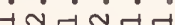

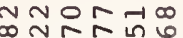
D능ㄴํㅇํㅇ ด 60606 $\circ 00000$

- 1 แ $10 \mathrm{~m}$ กูㄷํㅇำ 0 6 6 in $\circ 0^{\circ} \circ 0^{\circ}$

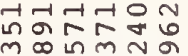

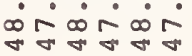

NAnTM in or nin ○ं0 000

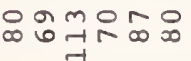

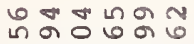
约得 月ँच $\dot{0} \sigma \dot{\sigma} \dot{\sim} \dot{m}$

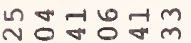
$\infty \infty \infty \dot{\infty} \infty \dot{\infty}$

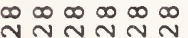

$0 \infty-N \rightarrow m$ เ ํํㄱㄷำ

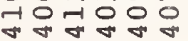

$m m \sim L N$ $\sim \infty \infty$ a m $\infty \cup+\infty \sigma \infty$ NลำสNN

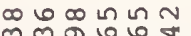
$\dot{0} \dot{0} \dot{\infty} \dot{\infty}$ 660606 $\neg \neg-\neg-\rightarrow$

HNm งัง $\infty \infty \infty \infty \infty \infty$ 동도옹 bronk O이잉 芯选芯 4⿻กงก $\begin{array}{lllll}1 & 1 & 1 & 1 & 1 \\ 2 & 1 & 1\end{array}$

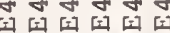

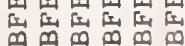

으은도 $\infty \dot{\infty} \dot{\infty}$

응ํำ 6060 $\circ 0^{\circ} \circ$

ㄴ $\ln \infty$ 등용 666 เी 0000

Thinch-1 n

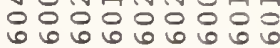
000000000

๓ก

$\infty \infty \infty \infty$ $\stackrel{\infty}{\sim} \stackrel{\infty}{\sim} \stackrel{\infty}{\sim} \stackrel{\infty}{\sim}$

오ำ네 ना mo กㄴ 0000

ดัनूก

T 0 No m $m \infty$ กำ

क กิ์ ำกำก

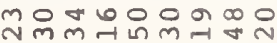

Nintarm

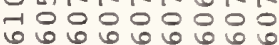
○ं0ல00000

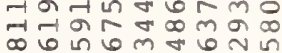

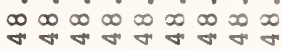

m내내유

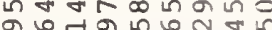
กิำำ 000000000

mm काष ๑ง 当 궣ㄱ

敞 $\infty \infty \infty \infty \infty \infty-\infty$

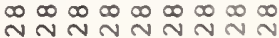

$\circ \infty \circ \sigma$ 얻ำ NกN

tha ด $\infty$ ก *nलmand

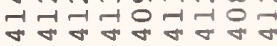

Nm-

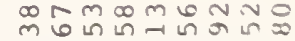

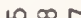
0000

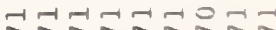
-1

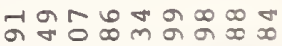
no $\operatorname{conNN}$ N

$\begin{array}{llll}1 & 1 & 1 & 1\end{array}$ $\infty \mathbb{N}_{\infty}^{\infty} \infty$

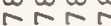
응ㅇㅇㅇ OOOO $m \infty \infty$ ก ก $\rightarrow$ o $\rightarrow d$

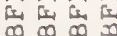

Nentangar

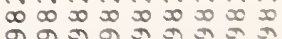

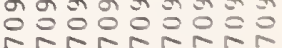
000050050

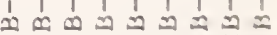
กละก

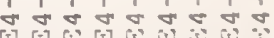

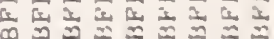
ง v v v

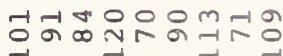




$\begin{array}{ccc}0 \\ 0 \\ -1 & 0 \\ -1 & 0 \\ -1 & 0 & 0 \\ 0 & 0 & 0 \\ 0 & 0\end{array}$

પ

己े

(1) मेल

फ

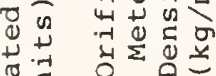

$\underset{\Xi}{0}$

$\underset{\pi}{\mathbb{C}} \stackrel{-1}{-1}$

ช

$\sum$

0
0

(1) 4

$\sum^{0} \cdot{ }^{1}$

iा

फ० 40

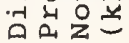

N

$\sum_{\substack{0 \\ 0}}^{0} \underset{0}{0}=$

$\therefore m$

萜

a

$\frac{a}{m}$

$\frac{1}{20}$

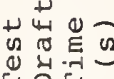

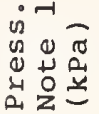

- $\dot{0} \dot{m} \dot{m} \dot{0}$

न

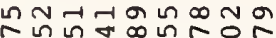

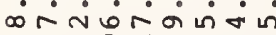

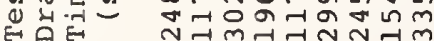

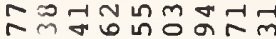

廿

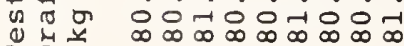

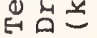

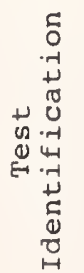

-1mㄴ

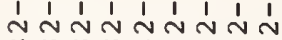
$\infty \infty \infty \infty \infty \infty \infty \infty \infty$ N $N \underset{N}{N} \sim N$

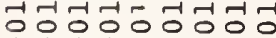
1 1 1 1 1 1

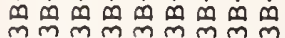

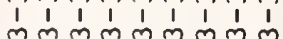

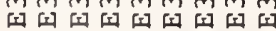

ก $m \infty \omega \sim \ln$

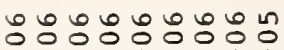

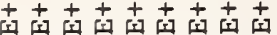

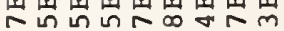

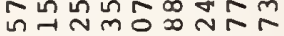
$\infty \pi m b$ ज

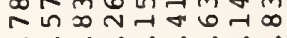

0 o ㄱํำ

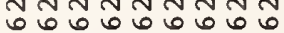
-00000000

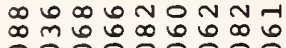

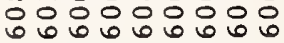
- $\dot{0} \dot{0} \dot{0} \dot{0} 0 \dot{0}$

an ติ సून $\dot{\sigma} \sim \sim \infty \infty \infty \infty \infty \infty \infty$

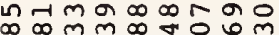

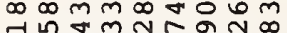
ที่ $\infty$ แี -ن்-i்

ำ $\infty$ ส

두음ำ

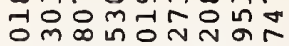
$\dot{\infty} \dot{0} \dot{0} \dot{\infty} \dot{\boldsymbol{N}} \dot{0}$

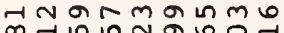
- $\cdot 0 \cdot \dot{0} \cdot$ i 0 ब ฟ $\sim \stackrel{\infty}{\sim} \stackrel{\infty}{\sim} \stackrel{\infty}{\sim} \stackrel{\infty}{\sim} \stackrel{\sim}{\sim}$

$\infty$ h $\dot{m} \dot{i} \dot{\sim} \dot{0} \dot{\sim} \dot{\sim}$

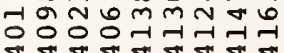

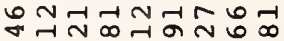
वंவ்ம் $\dot{0} \dot{0} \dot{0}$

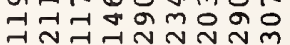

ด $000 m-1 n N$ -

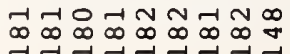

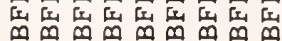

-

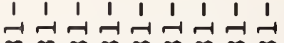

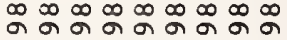

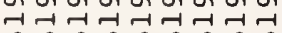
๒

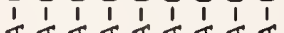

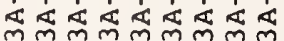

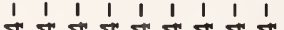
四四四四可可可

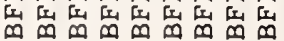
$\dot{\sim} \dot{\sim} \dot{\sim} \dot{A} \dot{-} \dot{-}$ 子न

융유유유

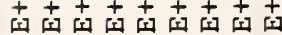
음ำ mㄱำ $\mathrm{m} \Omega$ 잉 Nown $\mathrm{N}$ m

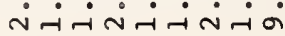

กลก m N N

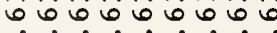
-00000000

क व $\mathrm{N} 0 \mathrm{H} \infty \infty 6$ न $6 乚 60606060$ 0ं0்000்

न

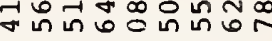
-

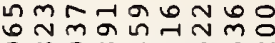
$0 \infty 0 \infty \infty \pi 0$ in

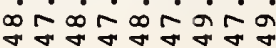

HNo

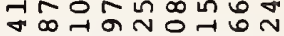
ningaron त०0नं00.

m

กตำกตก

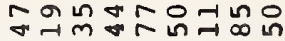
irirónón

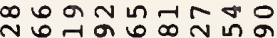
- $\cdot$ - $\cdot$.

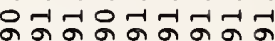
N $N$ N $N$ N

Nomm nก - $\dot{0} \dot{0} \dot{ } \dot{ } \dot{m} \dot{0}$ o

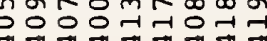

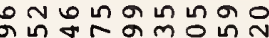
$\dot{0} \dot{0} \dot{0} \dot{0} \dot{0} \dot{0}$ जั बन

ด $0 m \omega-m \infty n$ moONROMNm ن $\dot{0} \dot{0} \dot{r} \dot{\sim} \dot{\sim}$

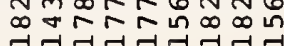

○

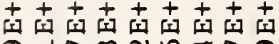
a드은 ㄱํㄱำ $\checkmark \sim \vartheta \sim$ - $\dot{\sim} \dot{\sim} \dot{\sim} \dot{\sim} \dot{\sigma}$

a

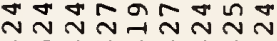
๑ - $\dot{0} \dot{0} \dot{0} \dot{0} \dot{0} \dot{0}$

$m m-16 m+\pi$ ๒

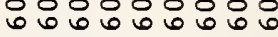
○ं00ं0000

A ดิ กัก ○ं०- $\dot{0} \dot{0} \dot{0}$

웅

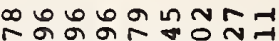

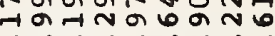

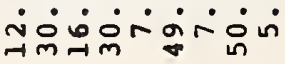

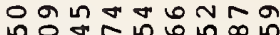

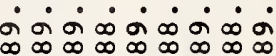
$\stackrel{\infty}{\sim} \stackrel{\infty}{\sim} \stackrel{\infty}{\sim} \stackrel{\infty}{\sim} \stackrel{\infty}{\sim} \stackrel{\infty}{\sim} \stackrel{\infty}{\sim} \sim \stackrel{\infty}{\sim} \stackrel{\infty}{\sim}$

a ant nmarm

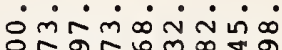

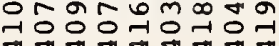

กmmnก

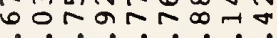

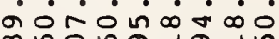

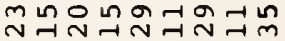

Don -

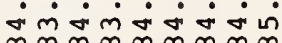
$\infty \quad \infty \quad \infty \quad \infty \quad \infty \quad \infty \quad \infty \quad \infty$

Tกm n $\begin{array}{ccccccccc}1 & 1 & 1 & 1 & 1 & 1 & 1 & 1 & 1 \\ -1 & -1 & -1 & -1 & -1 & -1 & -1 & -1 & -1\end{array}$ $\infty \infty \infty \infty \infty \infty \infty \infty$

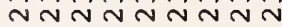

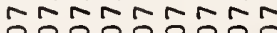

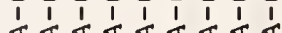

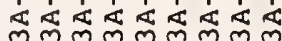

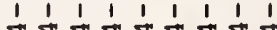

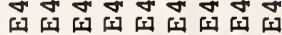

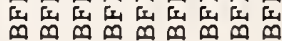

HNm n $\begin{array}{ccccccccc}1 & 1 & 1 & 1 & 1 & 1 & 1 & 1 & 1 \\ -1 & -1 & -1 & -1 & -1 & -1 & -1 & -1 & -1\end{array}$ $\infty-\infty)$ ไี

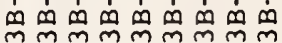

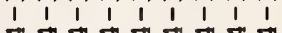

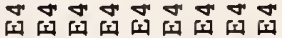

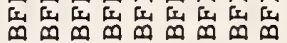

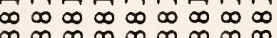
1 l l 110 
ஜ๐ㅇำ

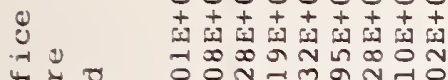

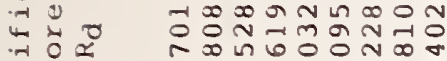
H. Non

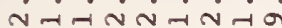

ง $\sim \sim \sim \sim \sim \sim \sim v$

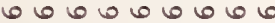
- 0000000

$m \pi 4 \pi 0000 \%$

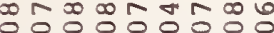
$\begin{array}{llllllllll} & 0 & 0 & 0 & 0 & 0 & 0 & 0 & 0 & 0 \\ 0 & 0 & 0 & 0 & 0 & 0 & 0\end{array}$ -0ं000000

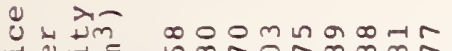
出光决

प थ . ? ? ? ?

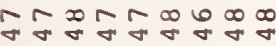

5

$\overbrace{}^{3}=$

n 3 म य० ल के है $\Sigma$ [थ $\simeq$ $-\dot{0} \cdot \dot{0} \cdot \dot{0}$

$$
\text { ए }
$$

กี

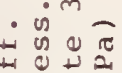

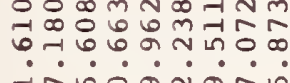
- $\begin{gathered}-1 \\ 0\end{gathered}$

mก

官芫

$\infty 6 N \in m+10$

.. . . ?

iे

ำ ำ ำ ำ ำ

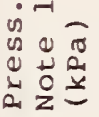

m $9 \mathrm{~mm}$ เก เก

Nمrí $\dot{0} \dot{0} \dot{0} 0$

0 。 $00 \pi$ n

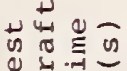

m N 0.

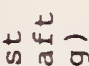

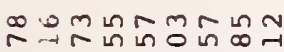

(1)

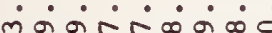

ㄱำ ขึก่

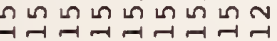

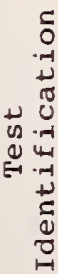

ศกm $\begin{array}{lllllllll}1 & 1 & 1 & 1 & 1 & 1 & 1 & 1 & 1\end{array}$

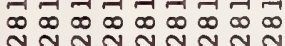
nNกNNNNNN

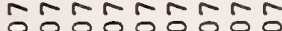
i 00000000

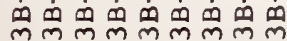

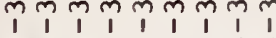

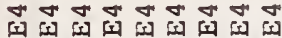

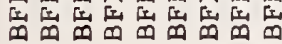

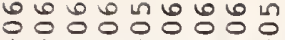
$+++++++$

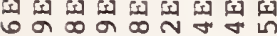
- 6 a

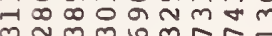
m m mmñ

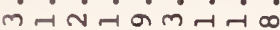

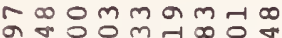

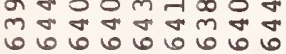
ல0ல0ல0000

On N I movm เกํํํํㅇํㅇํㅇำ

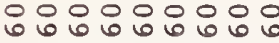
0ं00000.

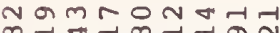

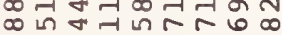

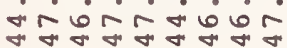

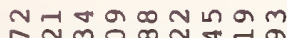
กิก 의 $\infty$ ํำ Nंศ்

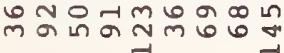

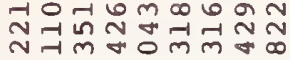

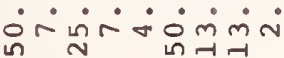

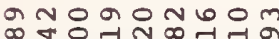
$\infty$ ㄴ.

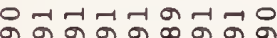

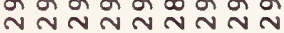

nto nam ant

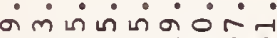

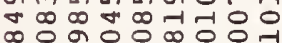

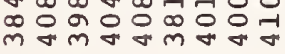

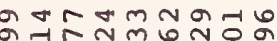
- . . - .

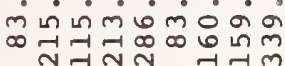

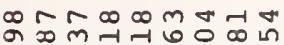
ம் $\dot{0} \dot{0} \dot{0} \dot{0}$

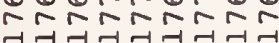

HNm ง

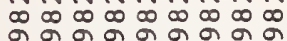

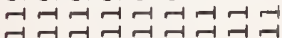
둥ㄷㅇㅇㅇㅇㅇㅇㅇㅇㅇㅇㅇㅇ

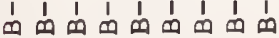

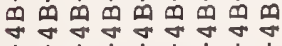
\begin{tabular}{llll}
1 & 1 \\
\hline & 1 & 1 & 1 \\
\hline
\end{tabular}

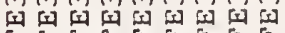

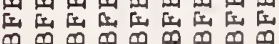

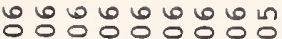
$++++t+++t$

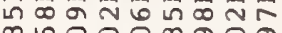

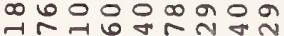

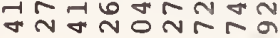

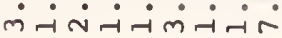

טNAN m ซ ○ं $000 \dot{0} 00$

겅ㅇㅇㅇㅛ D 읍 ல் $0 \dot{0} 0 \dot{0} 0$

은ㄷำกำ ฟึติำกำ $\dot{0} \dot{0} \dot{0} \dot{\sigma} \dot{0} \dot{\sigma}$

Mr

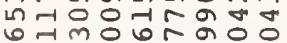
다 NOتंல்

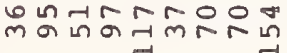

กำ aे $\infty-10$ in in 0 - in

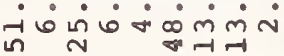

$\infty \nleftarrow \infty$ ก ก.?

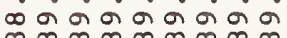

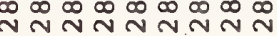

rmar mம் in $\infty$ in a ma a 絮

mกํำำ $\dot{m} \dot{-1} \dot{\sim} \dot{\sim} \dot{\sigma} \dot{\sigma} \dot{0}$

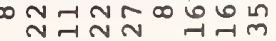

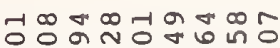

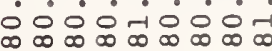

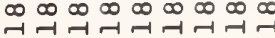

-1

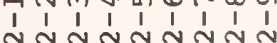

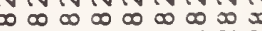

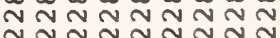
N N N N N N N N

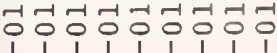

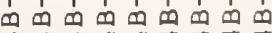

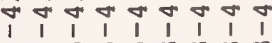
mल mm m mm

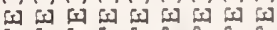

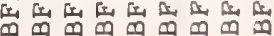

অm $\checkmark$ ฟ rodron

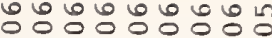

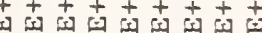
ฟ⿻ พื์ హิ

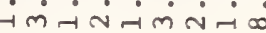

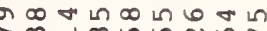

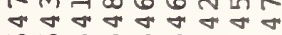
ซै ซु ซु ซु बु 000000000 ํㅣㅇㅢ 660666666 - 0000000

ำ

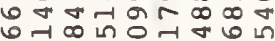

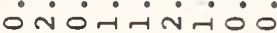

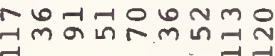

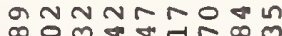

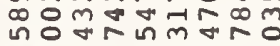

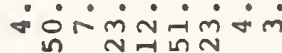

m

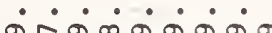
$\infty \infty \infty \infty \infty \infty \infty \infty \infty$

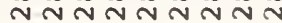

N $\infty \infty \infty \sim \infty \infty \infty$

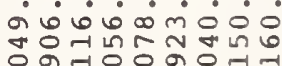
。

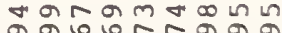
$\dot{0} \dot{0} \dot{0} \dot{0} \dot{0} \dot{0}$

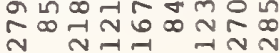

นก นกำกำ

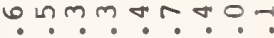

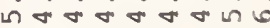
$\infty \infty \infty \infty \infty \infty \infty \cdots$

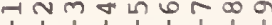

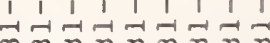

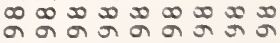
ซึ

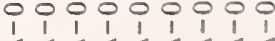

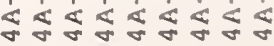

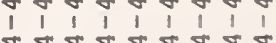
可

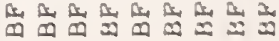

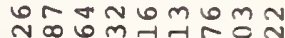




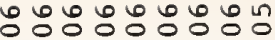

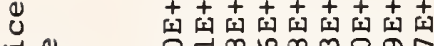

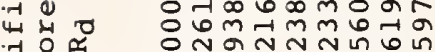

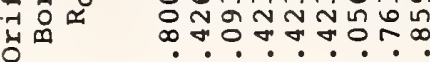

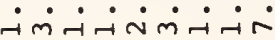

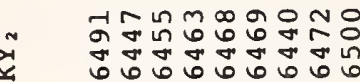
○ं

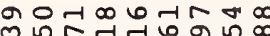

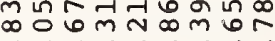
$\therefore \dot{0} \dot{0} \dot{0} \dot{0}-$

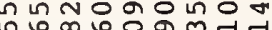

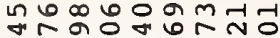

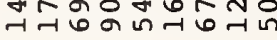
-i்

$\sigma^{\Sigma}$

\&

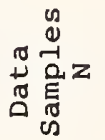

ำตㅇำ

$\dot{m}$

4ั

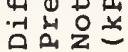

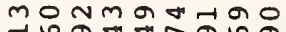
ที $\dot{r} \vec{\sim} \dot{\sim} \dot{\sim} \dot{\sim} \dot{\sim} \dot{\sim} \dot{\sim} \dot{\sim}$

之芯

ơ

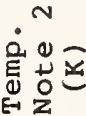

ดู่ ? नं०ं०नं० 00 $\stackrel{\infty}{\sim}$ ล

$\therefore m$

$\dot{\theta}^{-1}$

की

a.

onthatoon $\dot{\sigma} \dot{-1} \dot{0} \dot{-1} \dot{-1}$ mm

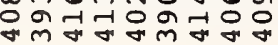

$\bar{m}$

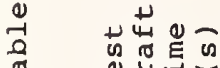

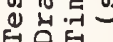

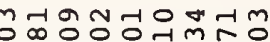

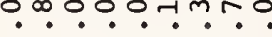
ㄴำ

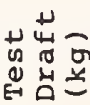

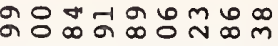
$\dot{\nabla} \dot{0} \dot{0} \dot{0} \dot{0} \dot{0} \dot{0}$ ซึ่

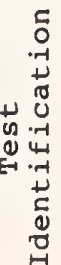

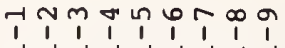

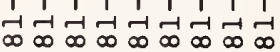

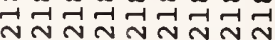
NNNNNNNNN

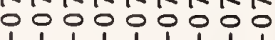
$\begin{array}{lllllllll}1 & 1 & 1 & 1 & 1 & 1 & 1 & 1\end{array}$

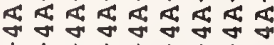

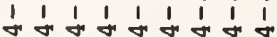

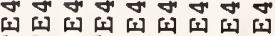

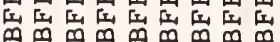

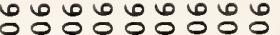
$++t+t+t+t$

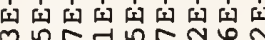
o 6 in mळ nm जn m० $\dot{m} \dot{i} \dot{-} \dot{i} \dot{m} \dot{-} \dot{-}$

○

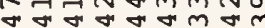

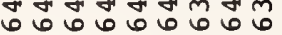
○ं0் 0000

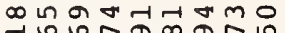
-

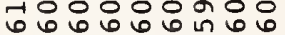

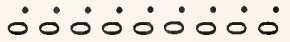

ㄴㅇㅇㅢ

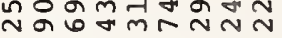
$\dot{\sigma} \dot{\sigma} \dot{\sigma} \dot{\sigma} \dot{i} \dot{\infty} \dot{\infty}$

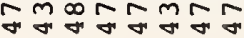

Nํ겅ㅇํำ응

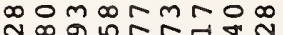

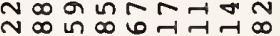

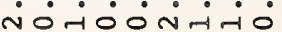

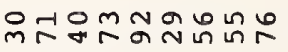

거요 에 $m$ 의

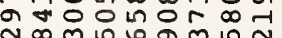

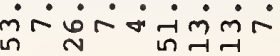

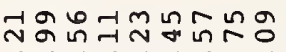
$\therefore \sim \pi \infty i r i \infty$ $\stackrel{\infty}{\sim} \stackrel{\infty}{\sim} \stackrel{\infty}{\sim} \stackrel{\infty}{\sim} \stackrel{\infty}{\sim} \stackrel{\infty}{\sim} \stackrel{\infty}{\sim} \stackrel{\infty}{\sim}$

○ $\pi \infty \pi$ a vi $\dot{\sim} \dot{\sim} \dot{\sim} \dot{\infty}$ สnत $\infty \times \infty \infty \sigma$

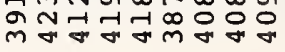

กำ ?. DNUNONHDサ g얻ำ

กิ $\dot{0} \dot{0} \dot{0} \dot{0} \dot{0}$

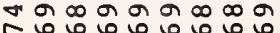

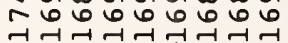

HNM ง

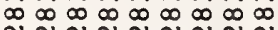
N

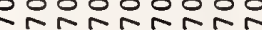
응ㅇㅇㅇㅇㅇㅇㅇ

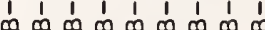

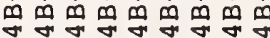

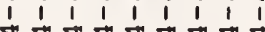

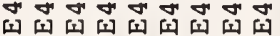

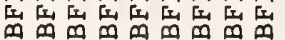

ฟึक

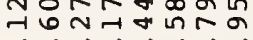
$\therefore \dot{0} \dot{0} \dot{0}$

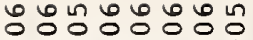
$t+t+t+t+$

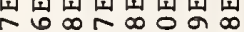
NRの6R in in N -iिंनंमें

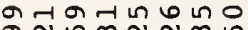

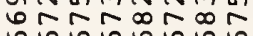
660606560 ○ं $\dot{0} \dot{0} \dot{0} \dot{0}$

$m m \infty N \infty \sim \infty$ טำ ㅇำ ○ं0 00000

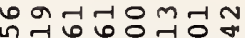

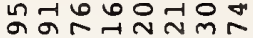
Nm -िं०नंत्र०

ต

Nm-16 กี $\dot{\sigma} \dot{m} \dot{\sim} \dot{\sigma} \dot{\dot{m}} \dot{\mathrm{N}}$

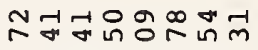
- $-\dot{-1} \dot{0} \dot{-1} \dot{-1}$

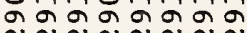
ก N N N N

a $\infty \dot{\infty} \dot{\sigma} \dot{-} \dot{-} \dot{\infty}$ m

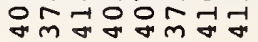

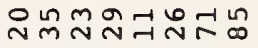
$\dot{ } \dot{i} \dot{\sigma} \dot{0} \dot{0} \dot{0}$

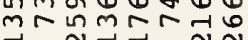

ㄷํำ in नㄷनㄷㄷㄷㄷ

TNm ส่ง

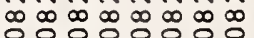

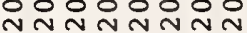

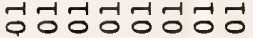

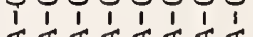

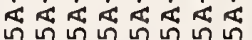
l 11 1 11 1 11 되되되 되되 되 되

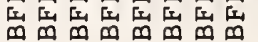

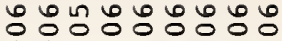
$+++++++++$

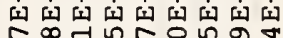
의

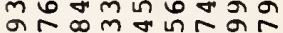

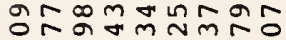
$\dot{-} \dot{-} \dot{\sim} \dot{\sim} \dot{m} \dot{-i} \dot{-}$

nTtmmang

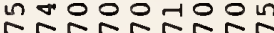
666566060 ○ं0 0ं $0 \dot{0} 0$

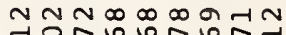
สํํํำด

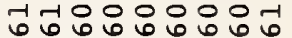
○ं 000 0ं

बm $9 N \infty m \infty \rightarrow$ I

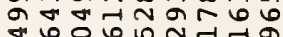
- 0.0. $\therefore \dot{\sigma} \dot{0} \dot{0} \dot{0} \dot{0} 0$

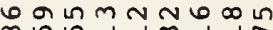

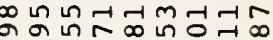

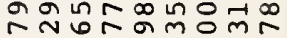
- $\dot{0} \dot{\circ} \dot{0} \dot{\sim} \dot{-}$

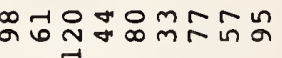

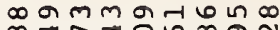

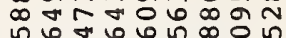
mं்

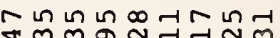
บ?ำ

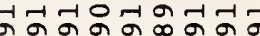
ขกำ ำกำ

ก Nمن் $\dot{0} \dot{\sigma} \dot{\sigma} \dot{\sigma}$

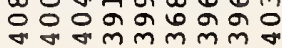

$\pi \pi 0, \pi N m$ ด नं $\dot{0} \dot{0} \dot{0}$ i N

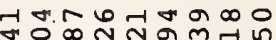
$\dot{m} \dot{m} \dot{m} \dot{m} \dot{0} \dot{0}$ $m m m m m \infty$ Un 0 $\infty \infty \infty \infty \infty N$

- $1 m \pi n \in \infty$ N $N \sim \mathcal{N} N$ $\infty \infty \infty \infty \infty \infty \infty \infty$ N

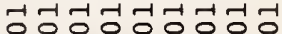
1,1 ต ต

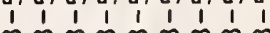
$m m m m m m m m n$

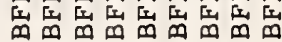


( )

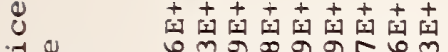
फٓ 我

$\infty m \sim \infty m_{m}^{\infty} \stackrel{n}{\sim}$

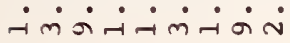

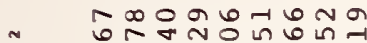 ㄱำ} $\therefore \circ 0.0 .0$

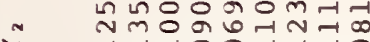

3

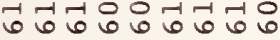
ல0்0ல0்0

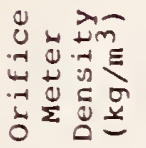

ํำ

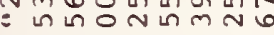
$\sim \dot{\infty} \dot{\infty} \dot{\sim} \dot{\infty} \dot{0}$ บ 恣 गत के 质光 m.

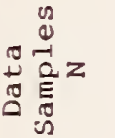

\&

- m $-\infty m \circ \nleftarrow m \infty n$

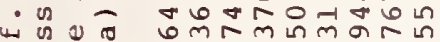
य 돌 कं

in

S

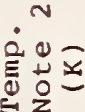

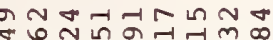
$\therefore \dot{0} \dot{0} \dot{0} \dot{0}$ $\infty \infty \infty \infty \infty \infty \infty \infty) \infty$

in

$\frac{0}{20}$

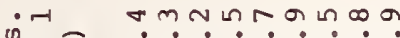
कि ब

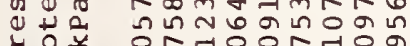

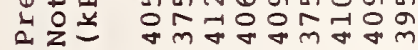

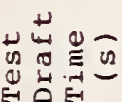

ம 6 m

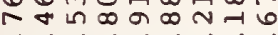

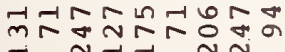

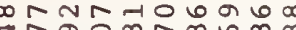

-

"1 出

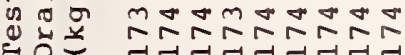
-

TNmF?

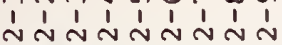
$\infty \quad \infty \quad \infty \quad \infty \quad \infty \quad \infty \quad \infty \quad \infty$

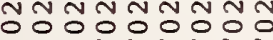

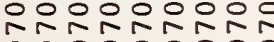
$\begin{array}{llllll}1 & 1 \\ 0 & 0 & 1 & 1 & 1 & 1\end{array}$

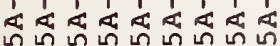
111111

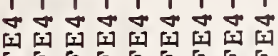

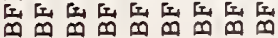
n $\operatorname{ar} 0$ 0

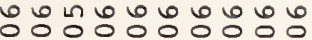

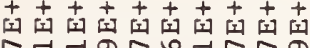
걷ำ क

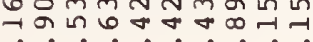

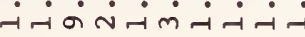

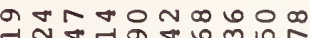
ทำ ติธตูอง ○ंல்ல் ல0ல் ஸ

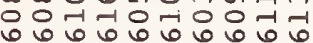
○ंல் $0 \dot{0} 0 \dot{0} 0$

นก⿻上丨 กี $\dot{\sigma} \dot{\sigma} \dot{0} \dot{0} \dot{0} \dot{\infty} \dot{\infty} \dot{\infty}$

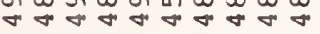

ก ळ $\dot{0} \dot{-1} \dot{-1} \dot{-1} \dot{-1}$

 N लं $\dot{\sim} \dot{\sim} \dot{0} \dot{0} \dot{0} \dot{m} \dot{m}$

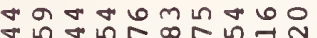
…ㄴ.? mannarim $\stackrel{\infty}{\sim} \stackrel{\infty}{\sim} \stackrel{\infty}{\sim} \stackrel{\infty}{\sim} \sim \infty \infty ⿻ \infty \sim_{\sim}^{\infty} \sim \infty$

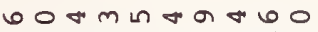

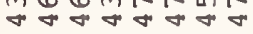

ம் O N

구인

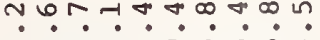
a듐ำ

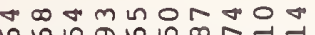
- 0. - 0 .

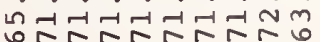

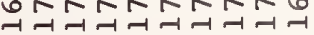

HNm ก.

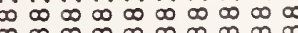

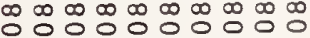

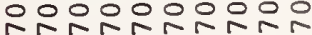
O000000000

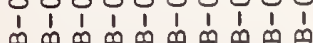

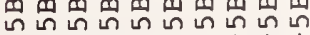
$\begin{array}{lllllllll}1 & 1 & 1 & 1 & 1 & 1 & 1 & 1 & 1\end{array}$

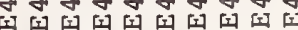

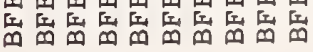

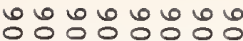
$+t+t+t+t$

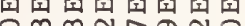
$+\infty$ in의 $m m$

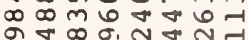
ผनं $\dot{\sim} \dot{-} \dot{\sim} \dot{-}$

$00 m \mathrm{r}$ - 0 \% กำกำ ninnNinTn ○0ல00000

GormoGn rratinga 으인이이이이인 О0ல00000

No부 6 6

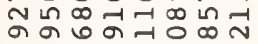
$\dot{m} \dot{0} \dot{0} \dot{r} \dot{r}$

능ํㅇำ न 정ำ

กี- $\sim-1-10$

m๒เ

- mor $00 \mathrm{~N}$

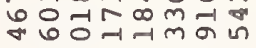
$\dot{\sigma} \dot{\sigma} \dot{0} \dot{0} \dot{0}$

다눙요 .

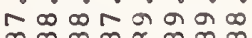

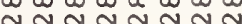

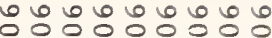
$++++++t+$

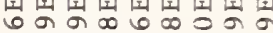
$m \infty \cup \infty r=00 \forall$

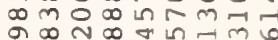

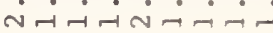

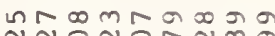
NNNNำกNำ nNMNNTNN000000000

Nadonthan คㅇํㅇㅇㅇㅇำ $\therefore \circ \dot{0} \dot{0} \dot{0}$

क- 0 N $\infty$ mmm $N$ ดु

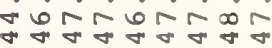

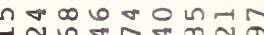
m O

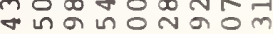
N-10-NmO

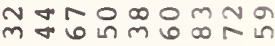

○ $m \sim \infty \infty \infty \omega m$

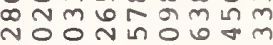
बíñن்

부의ำ

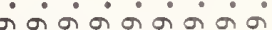

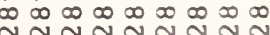

กดด

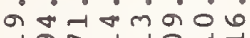

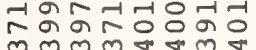

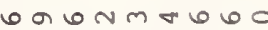
$\therefore \circ \dot{0} 0$ i

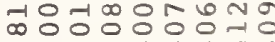

anm ?กต ㄴ.? $+\infty$ in $\infty$ N ปn一า

กำ융ำ மज र의

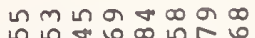
○0 00000

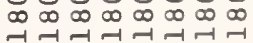

क ๙

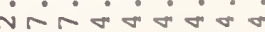
$\infty$ I ᄂ $\ln \infty \approx \infty \infty$

m๐n ง $\infty \infty \infty \infty \infty \infty \infty \infty$ สำสำสㄱํำ 더어더엉더엉 111111

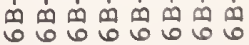
$\begin{array}{lllllll}1 & 1 & 1 & 1 & 1 & 1 & 1\end{array}$

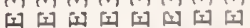

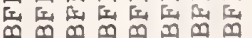

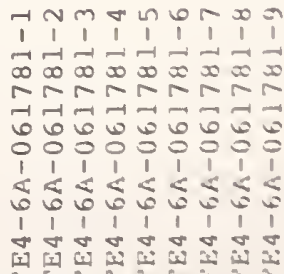

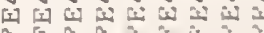

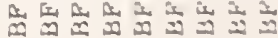


○ீீீீீீீீீ

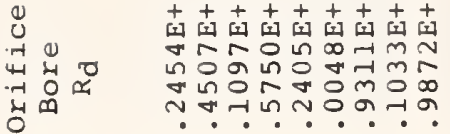
$\dot{i} \dot{i} \dot{-i} \dot{m} \dot{-i} \dot{i}$

त्र $\quad$ N

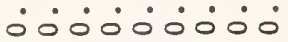

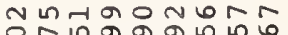

긴 ல் 000000

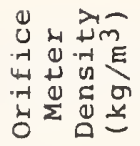

- $\infty$ n

ำ

$\dot{0}+\dot{0} \cdot \dot{0}$

$\infty n+\infty$

us 30

aำ

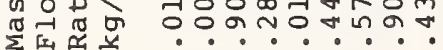
च iNondidin 萬

-in ${ }^{m}$ in

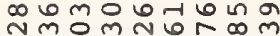

出 01 0 ㄱํำ - 4 出

n $m$ m $n-\infty \forall-1$

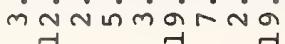

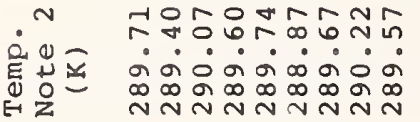

M

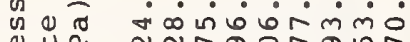

(1) NNRGONGUR

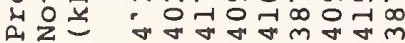

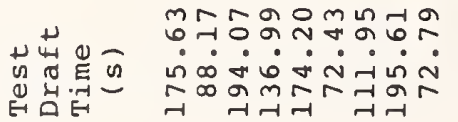

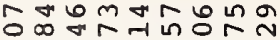

Drininir $\infty \infty \infty \infty \infty \infty \infty \infty \infty$

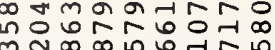
0.0 .0 $\infty \infty \forall r a \infty r o$

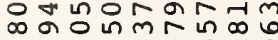

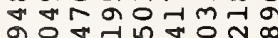
$\dot{0} \dot{\sim} \dot{\sim} \dot{-} \dot{\sim} \dot{-} \dot{-}$

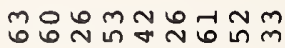

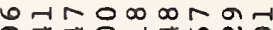
ดิ

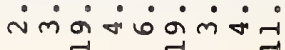

n tog nnmon ن $\dot{\sim} \dot{\sim} \dot{\sim} \dot{m} 0$

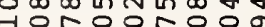
णm

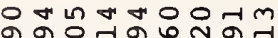

- 0.0 

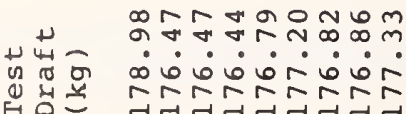

onnThRagn -1 $\infty-1, m \infty$ a ard $\infty$ in ก 60606060 નનનનનનનન્ન

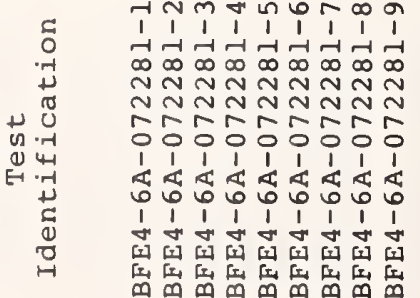

INm

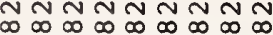
$\infty \infty \infty \infty$ ○。० 000000 STEOTONONOS

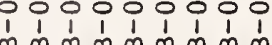
ตำ i 1,$1 ; \%$

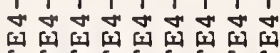

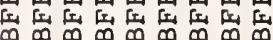


Table $4(a)$. Test Meter Identification and Physical Measurements Nominal 4 Inch AGA/API Orifice Meter (SI Units).

Test

Identification

BFE5-2A-060281-[9]

$\mathrm{BFE} 5-2 \mathrm{~A}-060581-[9]$

BFE 5-2A-080481-[7]

BFE5-2A-080581-[7]

BFE5-2B-060481-[10]

$\mathrm{BFE} 5-2 \mathrm{~B}-080581-[9]$

BFE5-2B-101581-[9]

$\mathrm{BFE} 6-2 \mathrm{~A}-052181-[11]$

BFE6-2B-052281-[10]

BFE6-2B-052881-[8]

BFE5-3A-060281-[9]

BFE5-3B-111281-[9]

BFE6-3A-051481-[10]

BFE6-3A-051581-[9]

BFE6-3A-052781-[8]

$\mathrm{BFE} 6-3 \mathrm{~A}-022582-$ [9]

BFE6-3B-022482- [ 9 ]

$B F E 5-4 A-080681-[9]$

BFE5-4A-101681-[10]

BFE5-4B-102281-[9]

BFE6-4A-102882- [9]

BFE6-4B-022582-[9]

BFE6-4B-100182-[9]

$\mathrm{BFE} 5-5 \mathrm{~A}-060381-[9]$

BFE5-5A-060481-[10]

$B F E 5-5 B-102081-[9]$

BFE6-5A-052781-[10]

BFE6-5A-052981-[1] ]

BFE6-5B-022482-[9]

BFE6-5B-092982-[7]

BFE 5-6A-080681-[9]

BFE5-6B-102081-[8]

BFE6-6A-102982-[9]

BFE6-6B-093082-[9]

BFE6-6B-100782-[9]

$\begin{array}{cccc}\text { Test } & \text { Run } & \text { Orifice } & \text { Beta } \\ \text { Configuration } & \begin{array}{c}\text { Diameter } \\ (\mathrm{cm})\end{array} & \begin{array}{c}\text { Diameter } \\ (\mathrm{cm})\end{array} & \text { Ratio }\end{array}$

1981-A

$1981-A$

1981-A

1981-A

1981-A

1981-A

$1981-\mathrm{A}$

1981-A

1981-A

1981-A

1981-A

1981-A

1981-A

1981-A

1981-A

1981-A

1981-A

1981-A

1981-A

1981-A

1981-A

1981-A

1981-A

1981-A

1981-A

1981-A

1981-A

1981-A

1981-A

1981-A

1981-A

1981-A

1981-A

1981-A

1981-A
10.217

10.217

10.217

10.217

10.217

10.217

10.217

10.226

10.226

10.226

10.217

10.217

10.226

10.226

10.226

10.226

10.226

10.217

10.217

10.217

10.226

10.226

10.226

10.217

10.217

10.217

10.226

10.226

10.226

10.226

10.217

10.217

10.226

10.226

10.226
3.8082

3. 8082

3. 8082

3. 8082

3.8090

3.8090

3. 8090

3. 8082

3. 8090

3.8090

0.3728

0.3728

0.3728

0.3728

0.3728

0.3728

0.3728

0.3724

0.3725

0.3725

5.0775

0.4970

5.0780

0.4970

5.0775

0.4965

5.0775

0.4965

5.0775

0.4965

5.0775

0.4965

5.0780

5.7127

0.4966

5.7127

5.7137

0.5592

0.5592

0.5593

\subsection{7}

0.5586

5.7137

5. 7137

0.5587

6.6660

0.5587

6.6660

0.6525

6.6670

0.6525

0.6526

6.6660

0.6519

6.6660

0.6519

6.6670

0.6520

6.6670

0.6520

7.6203

7.6182

7.6203

7. 6182

7.6182
0.7459

0.7457

0.7452

0.7450

0.7450

Note: Test Identification bracket gives number of tests run with this geometry. A total of 316 tests were run on the nominal 4 inch run size. 


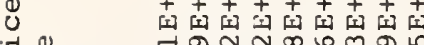
岱留 मू $\ddot{\sim} \dot{\sim} \dot{\sim} \dot{\sim} \dot{\sim} \dot{\sigma}$

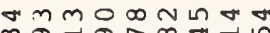

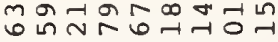
$\infty \pi \infty \infty \operatorname{son}$

ง 30 ज

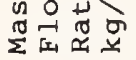

Gนแก

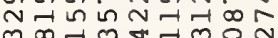

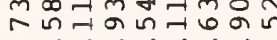

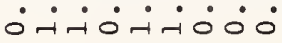
告

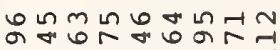
$\dot{n}^{m}$ 4 जा का $\rightarrow$ 出 0

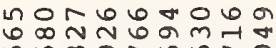

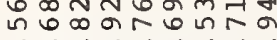

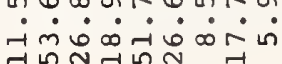

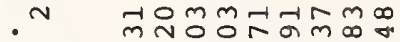
进 $\dot{\infty} \dot{\infty} \dot{\infty} \dot{\infty} \dot{\infty} \dot{\infty}$

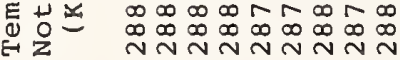

in $^{-1} 0006 \mathrm{nmoln}$ 纯 0 \%

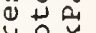

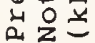

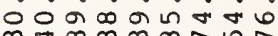

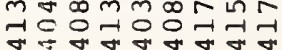

$\infty N N N \infty N \in R$ งี กัศ क०० שमㅐ

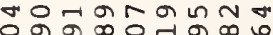
山 o

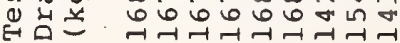

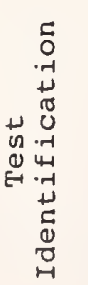

INm

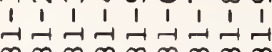
$\stackrel{\infty}{\sim} \stackrel{\infty}{\sim} \stackrel{\infty}{\sim} \stackrel{\infty}{\sim} \stackrel{\infty}{\sim} \stackrel{\infty}{\sim} \stackrel{\infty}{\sim} \stackrel{\infty}{\sim} \stackrel{\infty}{v}$

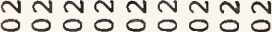
000606000 000000000

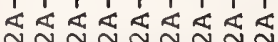
I I I I I 1

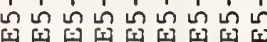

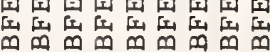

ஃே๐๐๐

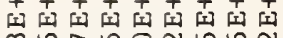
क लन $\dot{-} \dot{\sim} \dot{\sim} \dot{\sim} \dot{-} \dot{\sim} \dot{\infty}$

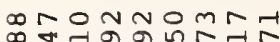

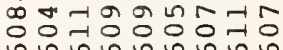
0600610610 0000000

कीनलmनचत ฟ

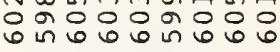
○ं0ல0ல0ல0

-1 $N$ N $\infty$-1mm $\infty$ नुल लि $\infty \infty r i \infty \dot{\infty} \dot{\infty}$

NกN $\infty \infty \infty \pi \infty$ 건 तU山 0ं- $-\dot{-10} \dot{0} \dot{0}$

ก에 $\infty$ ma 국

N กี ar. a d a n -iviñ

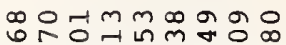
$\dot{\infty} \dot{\infty} \dot{\infty} \dot{\infty} \dot{\infty} \dot{\infty} \dot{\infty}$

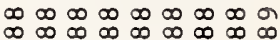
$\stackrel{\infty}{\sim} \stackrel{\infty}{\sim} \stackrel{\infty}{\sim} \stackrel{\infty}{\sim} \stackrel{\infty}{\sim} \stackrel{\infty}{\sim} \stackrel{\infty}{\sim} \stackrel{\infty}{\sim}$

antaroon on

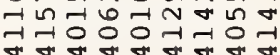

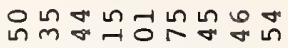
○ि $\dot{0} \dot{0} \dot{0} \dot{0} \dot{0} \dot{m}$

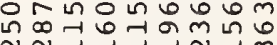

romaloman

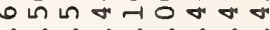

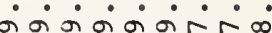
rarraras

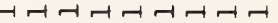

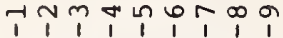

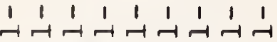

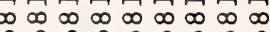

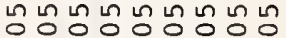
웅ㅇㅇㅇㅇㅇㅇㅇㅇㅇㅇ 웅ㅇㅇㅇㅇㅇ

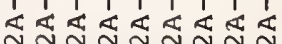

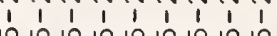
ก ᄂ

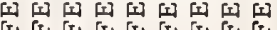

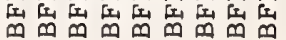

ஜ융유

$+++++t$

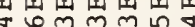
1 ㄴㅠㄸํ유 -i்- $\dot{\sim} \dot{\sim}$

$\infty-1$ 几 $\infty$ 几 几 ․․ㅇำ ๑ 6060606 $\circ \dot{0} \dot{0} \dot{0}$

बल की

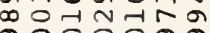
ติํํㅇㅇํㅇำ 0000000

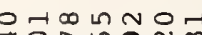
* 의 कorrín

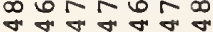

음ำ mㄴㅇㅛ NOOO विनं०्नं

nooran onn

$\infty a \infty \pi n$ M or $\infty$ - OON N

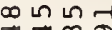
mNㅕำ i $\circ \dot{0} \dot{0}$ Inntinn กำ - $0 \dot{0} 00^{\circ}-$ ํํำำำ

RONALMN a ก

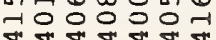

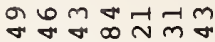
- $-\dot{0} 0 \dot{0} \dot{-1}-1$

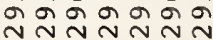

man no do

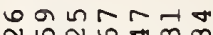

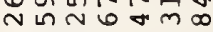

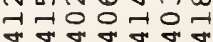

ดกNกM $0 m \infty \sigma \infty m$ $\dot{\sim} \dot{\sim} \dot{0} \dot{0} \dot{0}$

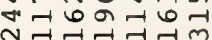

엄ำ

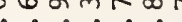
nonotor rarara

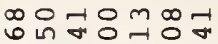
-ं் $\dot{\sim} \dot{\sim} \dot{\sigma} \dot{\sigma}$

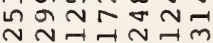

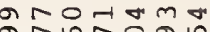
1. inNon $\infty \infty \infty \infty \infty \infty \infty$

11

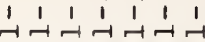
$\infty \infty \infty \infty \infty \infty \infty$ デすすずす $\infty \infty \infty \infty \infty \infty$ ○ 000000

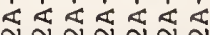
N 1 1 1111

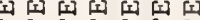

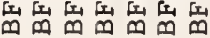

-1 N

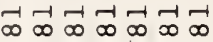

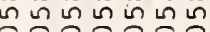
i 이웅ㅇㅇㅇ

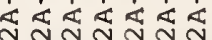
$\begin{array}{lllllll}1 & 1 & 1 & 1 & 1 & 1 & 1\end{array}$ เก็น

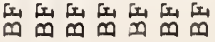




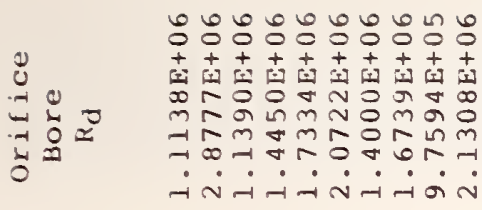

- $\quad$ añ

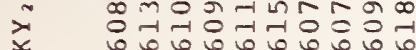
○ं0ं0்0000

ㄴำ य வ் $\dot{0} \dot{0} \dot{0} \dot{0} \dot{0}$

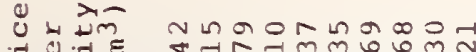

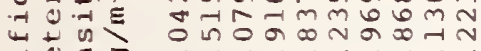
$\rightarrow$ U

ॠ

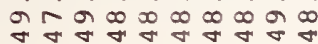
$\underset{0}{0}$

กभำ $\sum$ is 0- $\dot{0} \dot{0} \dot{0} \dot{0} \dot{0}-1$ गู

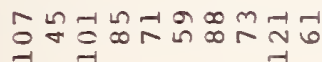
- is $\operatorname{in}^{m} 0$ $=0$

$m+N m-1 n \infty m$ L g.

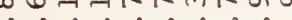

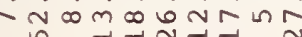

Uू

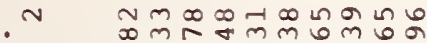
है⿴囗十 $\dot{0} \dot{\infty} \dot{\infty} \dot{\infty} \dot{\infty} \dot{\infty} \dot{\infty}$

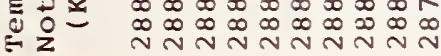

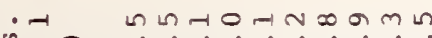

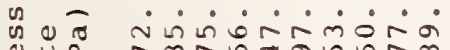

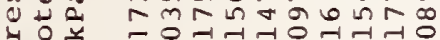
닌

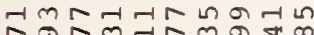
m $\stackrel{1}{=1}$

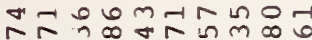

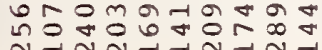
जंबंबंबंबंर เी

-

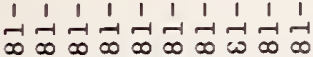
$\infty \infty \infty \infty \infty \infty \infty \omega \infty \infty$

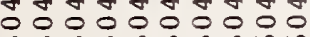
○ீ ๒

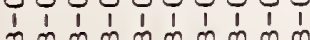

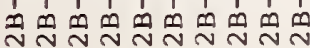
$\begin{array}{llllllllll}1 & 1 & 1 & 1 & 1 & 1 & 1 & 1 & 1 & 1\end{array}$

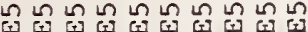

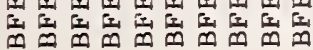

$\because 5: 0: 5005$

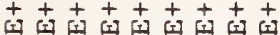

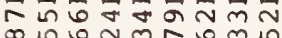

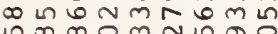
mू $\dot{-1} \dot{-i} \dot{\sim} \dot{-i} \dot{\sim} \dot{-}$

NG

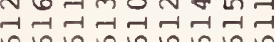

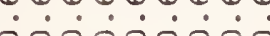

manomina

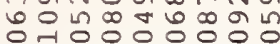

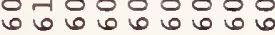
○ंல் $0 \dot{0} \dot{0}$

aำ-1 กำสูก - เा बm

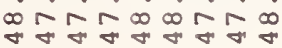

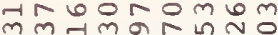
* $\infty \pi n \pi 0 N$ in * o ०म०-1000-10

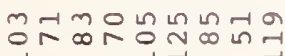

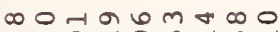

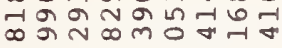
$\rightarrow \infty \infty$ in $\rightarrow \infty 0$

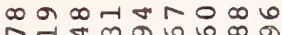

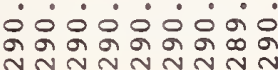

tanm⿻mnon $\therefore \dot{-} \dot{m} \dot{m} \dot{-} \dot{-} \dot{\sim}$ નૈ,

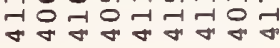

$\forall \forall \forall \infty \infty 0 m N 6$ ㄴ. -

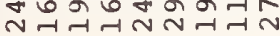

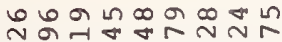
जंबंबंबनंबं

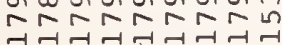

-1

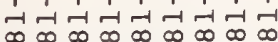
$\infty \infty \infty \infty \infty \infty \infty \infty$

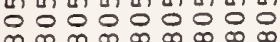

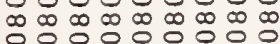
$\begin{array}{lllllllllll}1 & 1 & 1 & 1 & 1 & 1 & 1 & 1 & 1\end{array}$ ํํㄹ $\stackrel{m}{\sim} \stackrel{m}{\sim} \stackrel{m}{\sim} \stackrel{m}{\sim}$ $\begin{array}{lllllllll}1 & 1 & 1 & 1 & 1 & 1 & 1 & 1 & 1\end{array}$

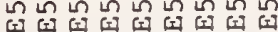

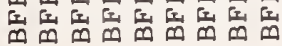

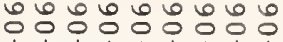
$++t+t+t+$

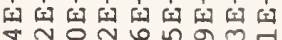
$\infty \forall$ ช

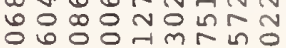
v- $\dot{\sim} \dot{-1} \dot{\sim} \dot{-}$

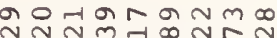

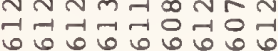
$\therefore \circ 0 \circ 0.000$

बनतथक्णm

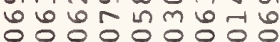

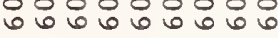
வல் $\dot{0} \dot{0} \dot{\circ}$

อนก กิำ

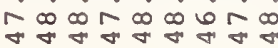

O $N \infty N$ m non $\infty 6 m \neq 6-10 \infty \infty$ 슨 -i்-i் $\dot{0} \dot{0} 0$

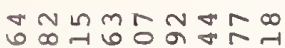

요문 ชิ

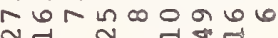

ㅇํำํํㄱํํ융 ○ं 00000 नून नू बू क

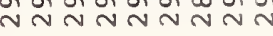

$\infty$ แก ᄂ O

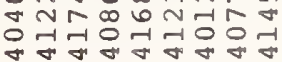

ตㄴ $\ddot{\sim} \dot{0} \dot{0} \dot{0} \dot{0}$ สู่

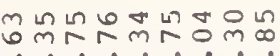
$\infty \dot{\sigma} \dot{\circ} \dot{m} \dot{m} \dot{ }$ 0 แ

HNm $*$ L 0 \%

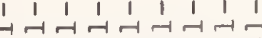

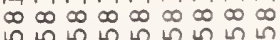
덩더엉더어엉 1ૈન્1

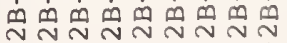

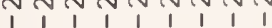

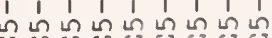

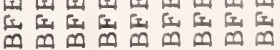

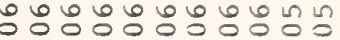
$++t+t+t+t+t+t$ $N \backsim \infty m+N \infty \omega N N$

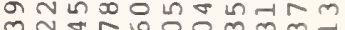
m ผ

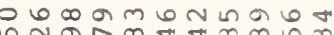
ת 00000000000

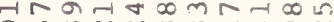

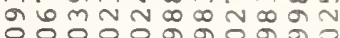
60606 in in 6 in in ○ं00000000

m

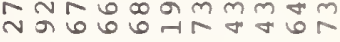

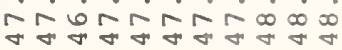

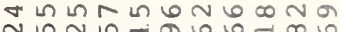
NกN

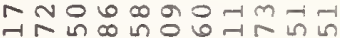
-i்-i0்-10-100

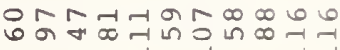

แ लन जूत g-

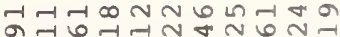
ํํำลำลำลำ

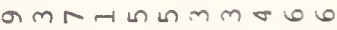

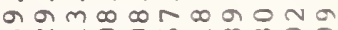

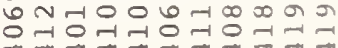

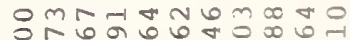
$\dot{m}-1$ m no no in ปึ

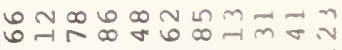

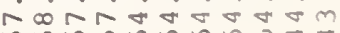

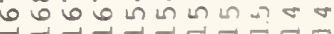

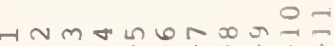

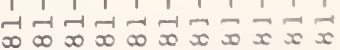
ปีสำสสำสำสำ

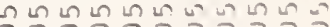

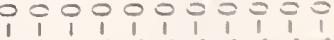

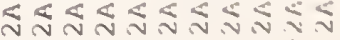
o 606000

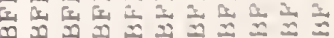




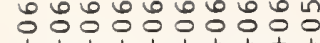

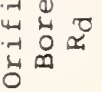

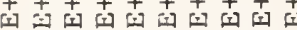
त-1िक

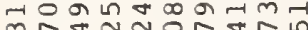

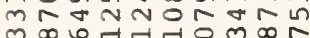

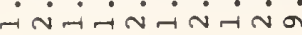

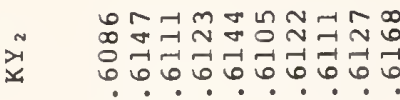
ல் $\dot{0} \dot{0} \dot{0} \dot{0} 0$

0 4 से 出出

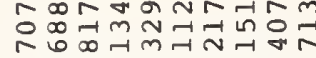

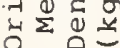

نे

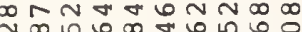

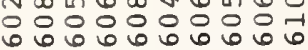
$\infty \sim \infty \sigma \infty \sigma \infty \pi$

\section{(0)}

䓎

is 300

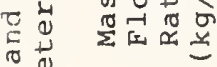

$\tau$

(1)

$\underset{3}{3} \cup$

(2) 4

$\sum \stackrel{4}{0}$

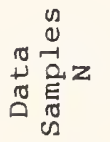

बै

m๒

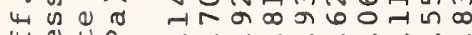
4 40 年出

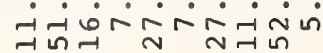

유ำ

N N ○म் $\dot{0} \dot{\circ} \dot{0} \dot{-0} \dot{0}$

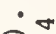

范

O̊N

न

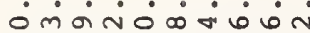
NMNGDINRTN

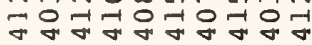
ம் $\dot{0} \dot{0} \dot{\sim} \dot{r} \dot{0} \dot{0}$ กำ

유픔ำ 山出 4 过苟先 告) Tกm

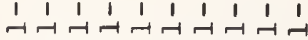
$\infty \quad \infty \quad \infty \quad \infty \quad \infty \quad \infty \quad \infty \quad \infty \quad \infty \quad \infty$

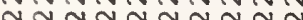
แn 1000000000 $\begin{array}{llllllllll}1 & 1 & 1 & 1 & 1 & 1 & 1 & 1 & 1 & 1\end{array}$

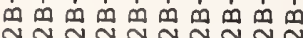
$\begin{array}{llllllllllll}1 & 1 & 1 & 1 & 1 & 1 & 1 & 1 & 1 & 1\end{array}$ 0600000000

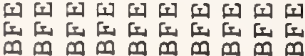

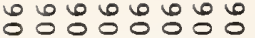
$++t+t+t+$

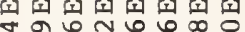

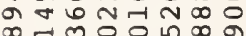
œ $\dot{\sim} \dot{\sim} \dot{-} \dot{\sim} \dot{\sim}-\dot{ }$

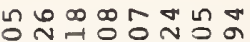

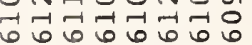
$\therefore \circ \circ \circ \circ \circ$

ช * 으잉ㅇㅇㅇㅇㅇㅇㅇㅇㅇㅇㅇ - 000000

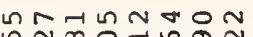
ก กับ $\dot{\infty} \dot{\infty} \infty \sim \sim \infty$

ำ

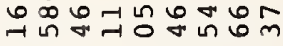
$\dot{\infty} \dot{\infty} \dot{\infty} \dot{\sim} \dot{\infty} \dot{\theta}$

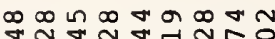
N N $N \mathcal{N} N$ N 0 00606000 ○ं $0 \dot{0} 0 \dot{0} 0 \dot{0}$

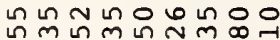
웅ㅇㅇㅇㅇㅇㅇㅇㅇㅇㅇㅇㅇㅇ ○ं0 00000

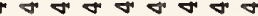

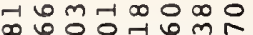
न ○ं०் $0 \dot{0}-\dot{1}$

œ

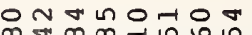
on

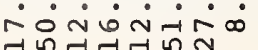

- annmán H. $\infty \sim \infty \sim \infty \dot{\infty} \infty$ $\infty \underset{\infty}{\infty} \infty \infty_{\infty}^{\infty} \infty \infty \infty \infty$

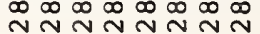

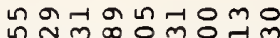
Unष mor $\infty$ m -0்0-

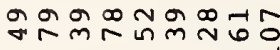

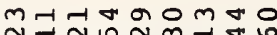

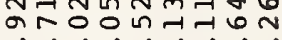

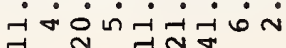

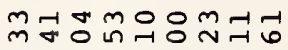
$\dot{\infty} \dot{\infty} \dot{\infty} \dot{\infty} \dot{\infty} \dot{\infty} \dot{\infty} \dot{\infty}$

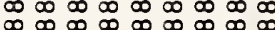

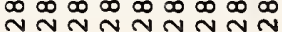

Nm $\dot{\sim} \dot{\sim}$ i்

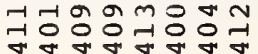

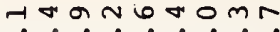
ondoñon anv 읭웡ㅇㅇㅇㅢ 익

年年4 o ஸिं ำ

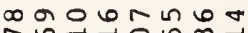
6 $\infty$ in $\infty$ o \& in जी in

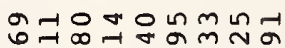
$\therefore \dot{0} \dot{\sim} \dot{m} \dot{-} \dot{0} \dot{0}$

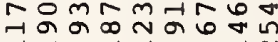

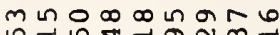
กี जीन

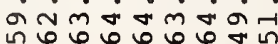

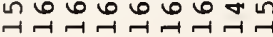

$\begin{array}{lccccccc}1 & 1 & 1 & 1 & 1 & 1 & 1 & 1 \\ -1 & -1 & -1 & -1 & -1 & -1 & -1 & -1\end{array}$ $\infty \infty \infty \infty \infty \infty \infty$

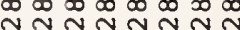

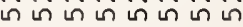
잉ㅇㅇㅇㅇ

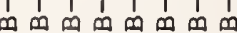

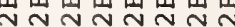
$\begin{array}{lllllllll}1 & 1 & 1 & 1 & 1 & 1 & 1 & 1\end{array}$

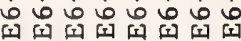

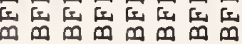

Hพm

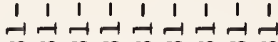

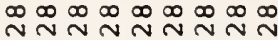
○0 0000000

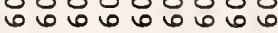
o 00 o 0 O 00

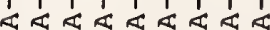

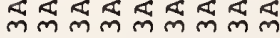

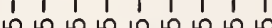

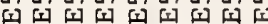

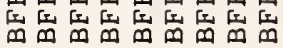

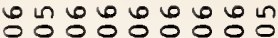
南南南南南南南南南 m

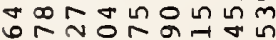
बूँ

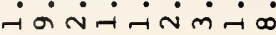

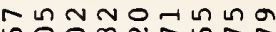

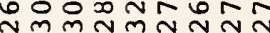
สูก กิก กิก :

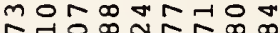
เำ

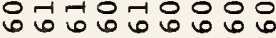
ல0்0ல000

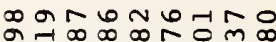
ᄀนทั

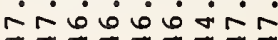

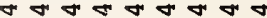

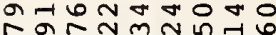
กิ

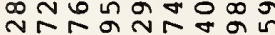
-نं $\dot{0} \dot{0} \dot{\sim} \dot{0}$

윰요

anm ONNNH a न $\dot{\circ} \dot{\sim} \dot{\sim} \dot{0} \dot{0} \dot{\sim} \dot{\sim}$

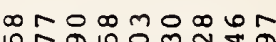

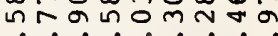
-1 -

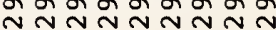

म0नामHल का

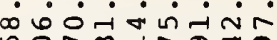

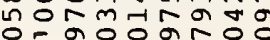
의읭의의

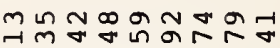
$\dot{0} \dot{0} \dot{-i} \dot{\sim} \dot{\sim} \dot{\sim}-\dot{r}$

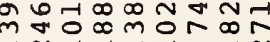

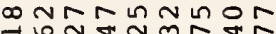
- . . . . . .

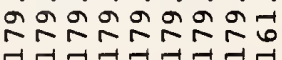

- 1 m $\begin{array}{ccccccccc}1 & 1 & 1 & 1 & 1 & 1 & 1 & 1 & 1 \\ -1 & -1 & -1 & -1 & -1 & -1 & -1 & -1 & -1\end{array}$ $\infty \infty \infty \infty \infty \infty \infty \infty$

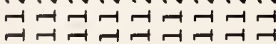

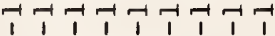
品品品品品 $\begin{array}{llllllllll}1 & 1 & 1 & 1 & 1 & 1 & 1 & 1 & 1\end{array}$

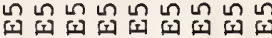

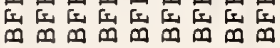




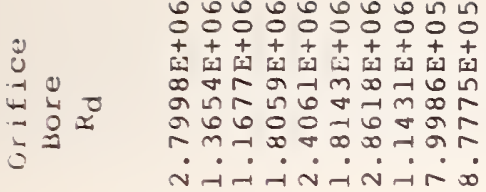

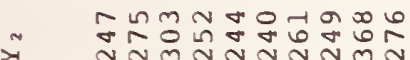

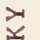

 ๑ 6 6 6 6 6060 0000000000

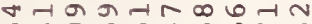
n

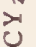
ใู $\therefore \circ 00000000$

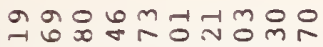
no a N $\mathrm{N}$ in un in

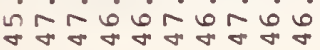

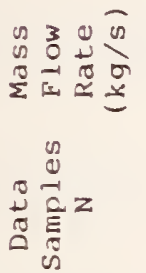

$\infty N-\infty \forall-1 \infty \infty \infty$ F จू⿻

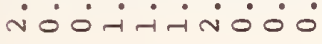

ํำ

- m

$\therefore$ in 0

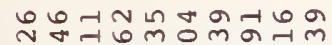
ข้ีำ

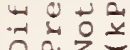

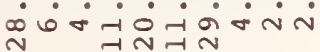

สำ a $\infty \infty \times 2$. $=2$

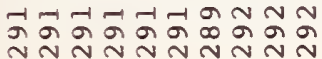

Non

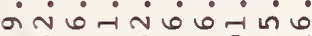
जิ a $z$

×

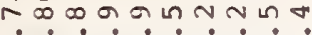

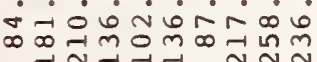

인 is $\frac{4}{0} \bar{\sigma}$

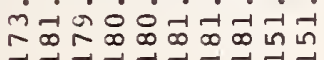
넌

HNm n

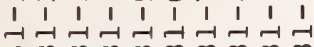

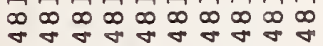

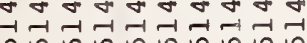
กิ์ $\begin{array}{llllllllll}0 & 0 & 0 & 0 & 0 & 0 & 0 & 0 & 0 & 0 \\ 1 & 1 & 1 & 1 & 1 & 1 & 1 & 1 & 1 & 1\end{array}$

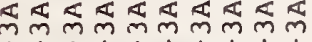
$\begin{array}{llllllllll}1 & 1 & 1 & 1 & 1 & 1 & 1 & 1 & 1 & 1\end{array}$

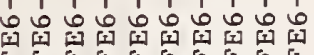

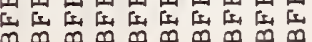

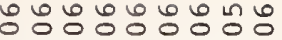
$+++++++t+$ ㅊำ ชำ लำ ज्ञ $\dot{-} \dot{-1} \dot{\sim} \dot{\sim} \dot{\sim} \dot{\sim}$

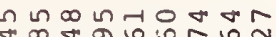
ง ขึง ำ ด ชิ ชู - 0000000

거네대 000.10000000

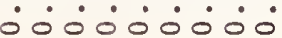

o $\operatorname{con}$ ก

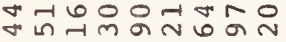
$\dot{\sigma} \dot{\sigma} \dot{\sigma} \dot{\sigma} \dot{\sigma} \dot{\sigma} \dot{\sigma} \dot{\sigma}$

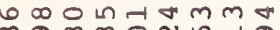

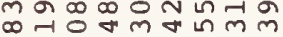
ڤొळ ○ं $\dot{-1} \dot{-} \dot{0} \dot{0} \dot{\circ}$

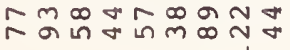

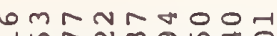

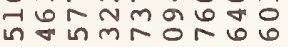

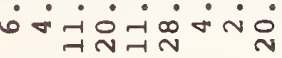

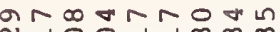

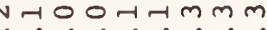

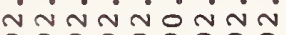
ขิำกำสำกำ

ด $\therefore \dot{\infty} \dot{\sim} \dot{\sim} \dot{0} \dot{0}$ O슌 의의의

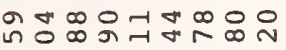
$\therefore \sim \dot{\sim} \dot{\sim} \dot{0} \dot{0} \dot{0}$ - N

กต느요

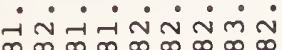

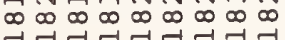

Nm+n $\begin{array}{ccccccccc}1 & 1 & 1 & 1 & 1 & 1 & 1 & 1 & 1 \\ -1 & -1 & -1 & -1 & -1 & -1 & -1 & -1 & -1\end{array}$

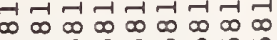
ก ถึกี่ i 1011111

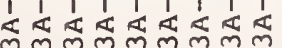
$\begin{array}{lllllllll}1 & 1 & 1 & 1 & 1 & 1 & 1 & 1 & 1\end{array}$

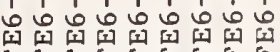

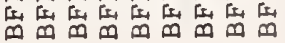

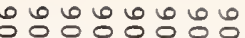
十南南+十南+十

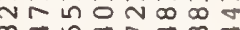
$\infty \sigma \omega \sigma \sigma m$

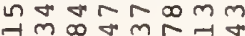
$\dot{-i} \dot{i} \dot{\sim} \dot{-} \dot{-} \dot{\sim}$

\% ง $\stackrel{\infty}{\sim} \stackrel{\sim}{\sim}$ ㄴำ

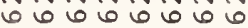
ல0் 00000

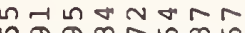
ก

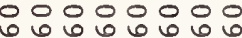
○்

-

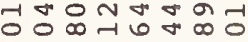
obrarri

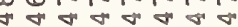

- nNamU

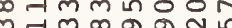

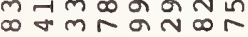
$\dot{0} \dot{-1} \dot{-} \dot{0} \dot{0}$

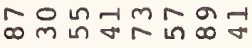

omnmagn aranman ก $\dot{\sim} \dot{m} \overrightarrow{-} \dot{-} \dot{0} \dot{0} \dot{\circ}$

ก $m \varphi m o n \varphi 6$ dramarn $\infty \stackrel{\infty}{\infty} \infty \infty_{\infty}^{\infty} \infty \infty \infty$

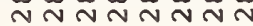

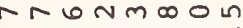
$\because \dot{\sigma} \infty \infty \dot{\sim} \dot{\infty}$ ă 잉

o बm-n -? L? ?

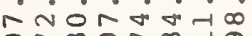

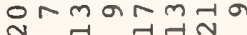

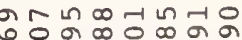
$\dot{\sim} \dot{\sim} \dot{\sim} \dot{\sim} \dot{m} \dot{m}$ añ

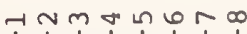

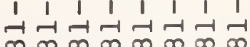
r

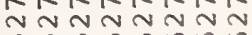
ถึก O 0000000

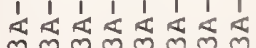
$\begin{array}{llllllll}1 & 1 & 1 & 1 & 1 & 1 & 1 & 1\end{array}$

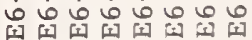

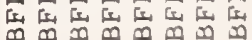

NmLIOROAR

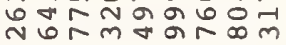
- $\dot{\sim} \dot{\sim} \dot{0} \dot{\sim} \dot{\sim} \dot{\sim}$

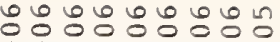
$++t+t+t+t+$ orman N

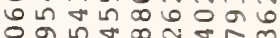
$\infty$ momm $\neg m \rightarrow m \sim \neg \sim \infty$ ขึ)

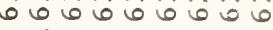
000000000

NGน-1n

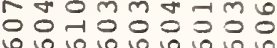
ல் 000000

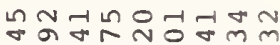
nmon

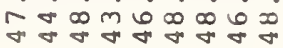

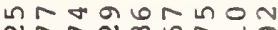

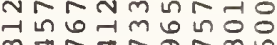
m机 - NoN-100-10

చืल

เmตบํㅇำ $\cdot \cdot \dot{0} \dot{0} \dot{0} \dot{0}$

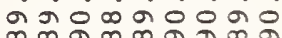
脑

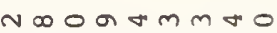

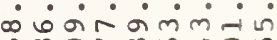
ก

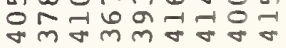

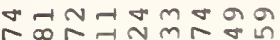
-iviricio araำ

ำกำสำ영 $\dot{\omega} \dot{\omega} \dot{\omega} \dot{0} \dot{\sigma} \dot{\sigma}$

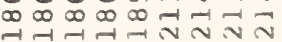

- 1 m

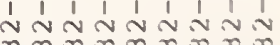
$\infty \infty \infty \infty \infty \infty \infty \infty \infty$ N N $\mathcal{N} \sim \mathcal{N} \sim \mathcal{N}$

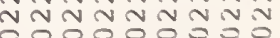
$\begin{array}{llllllll}1 & 1 & 1 & 1 & 1 & 1 & 1 & 1\end{array}$

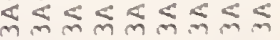
$6000 \omega$ I

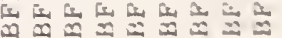

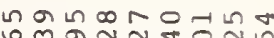




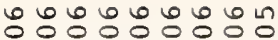
Ð $-40$

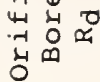

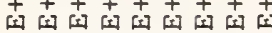
$\rightarrow m$ 约细 $\pi m 0 \omega \infty \cdots$ ○ल $\dot{-i} \dot{-i} \dot{-i} \dot{\sim} \dot{\infty}$

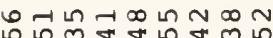

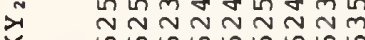
$\underset{1}{x}$

๑ ம் $\dot{0} 0 \dot{0} \dot{0} 0$

min 웅ㅇㅇㅇㅇㅇㅇ은 0ं000000

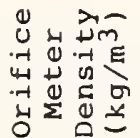

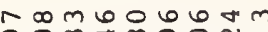

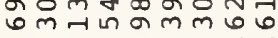
$\therefore \dot{0} 0 \dot{0}$

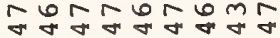

os 300

4R⿻上丨

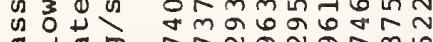

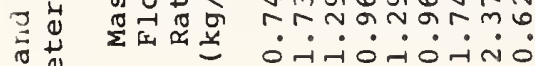
$\left.{ }^{0}\right)^{0}$ 先

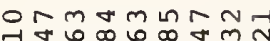

की

i

궁

$\dot{m}$

- iो is o

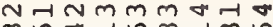

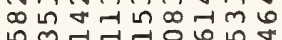
$\dot{m} \dot{0} \dot{0} \dot{0} \dot{0} \dot{0}$

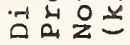

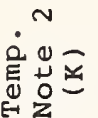

$\infty m m \in \sim \sim \sim \infty$ in

$H$

$\because \infty-6 \infty$ बा $\rightarrow \dot{0} \dot{0} \dot{-1} \dot{\sigma} \dot{\sim}$ $\infty \cos$

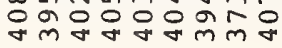

ำ $0 N \infty \pi m$ ก.

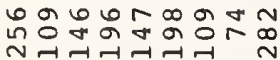

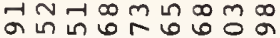
山出

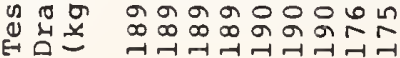

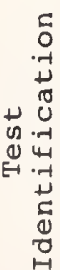

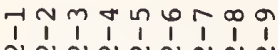

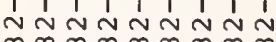

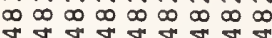
N N N N N N nNanNnNNN o 00000000

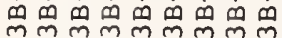
b' 1

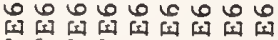

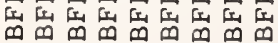

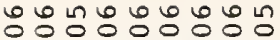

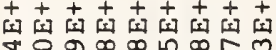
N ก In ก⿻

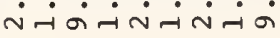

in $\infty \pi \infty \infty$ L $\infty$ an ஸิ

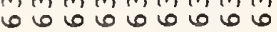
¿0ं0்0ல்

ํㅏㅇㅢ

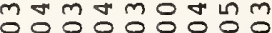

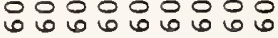
웅ㅇㅇㅇ

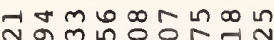

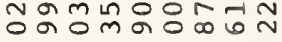
$\therefore \dot{\sigma} \dot{\sigma} \dot{\sigma} \dot{\sigma} \dot{\sigma} \dot{\sigma}$

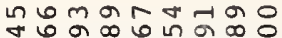
ง బ i० $\dot{0}$ i

궁요

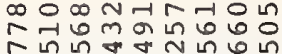
$\dot{m} \dot{\sim} \dot{\sim} \dot{m} \dot{m} \dot{\sim}$

ฟิm

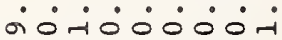

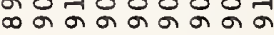
ำกำกำ ำ

Ho

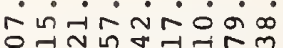

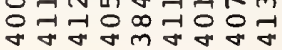

mนก ᄂ ดन ब ம் $\dot{0} \dot{0} \dot{\sigma} \dot{\sim} \dot{0}$

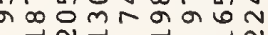

ㅇำ $-\dot{0} \dot{0} \dot{0} \dot{0} \dot{0}$

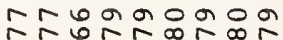

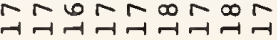

T1 卢 000000000

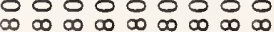
更 000

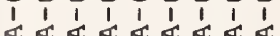

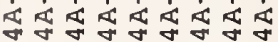

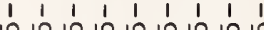

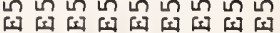

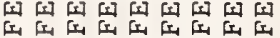

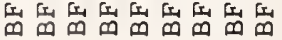

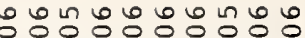

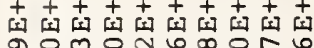

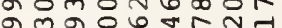
ำ $\rightarrow \infty 0$ त

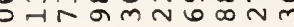
$\dot{m} \dot{\sigma} \dot{\sim} \dot{\sim} \dot{\sim} \dot{\sigma} \dot{\sim}$

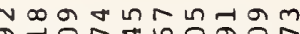

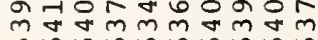

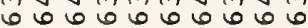
○ं0ல்00000

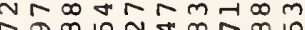

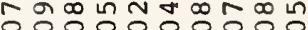

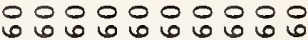
ல0்0ல0் 000

Hनتย

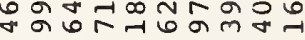

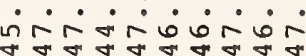

-

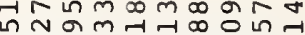

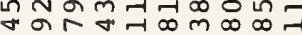
vं0் $\dot{0} \dot{-1} \dot{0} \dot{-} \dot{-}$

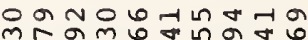

m ขै $\dot{\sim} \dot{\sim} \dot{\sim} \dot{\sim} \dot{\sim} \dot{\sim} \dot{\mathrm{\sigma}}$

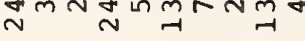

ต 月नก m

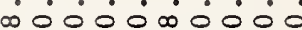
\%

moo a m an a m 6 - $\dot{\sim} \dot{0} \dot{0} \dot{0} \dot{0} \dot{m}$ ron nmb

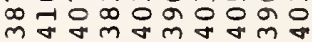

$\infty$ เ กง ○ a

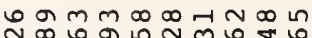
ن $\dot{1} \dot{\sim} \dot{\sim} \dot{\sim} \dot{\infty} \dot{\infty}$ NลースNN

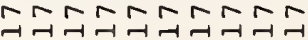

- 1 ก

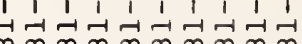

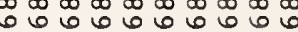

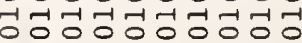

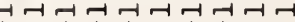

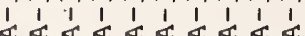
质芯芯芯芯芯芯

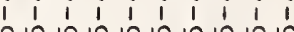

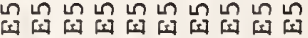

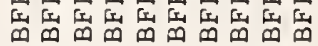

○ัน์ $++++++++t$

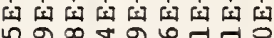
$\checkmark-m$ in 060 -

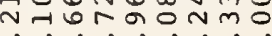
ง

NN" 능요

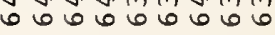
○ं0ல00000

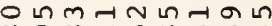
œの

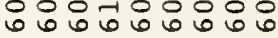
$\therefore \circ \dot{0} 00000$

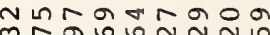
Mกลด orararog

I GOGMRNOR mำㄴำㄴำ

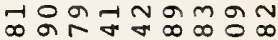

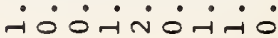

ำกำกำำㄴำ

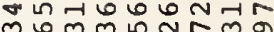
ข้ำ $\dot{m} \dot{\sim} \dot{\sim} \dot{\sim} \dot{m} \dot{\sim}$

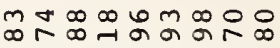
$\therefore \dot{0} \dot{0} \dot{0} \dot{0}$

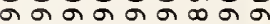

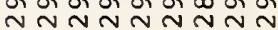

$\infty m \circ$ an $N \infty \infty$ Ln $\dot{0} \dot{\omega} \dot{\sim} \dot{\sim} \dot{\omega} \dot{0}$

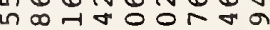

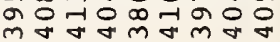

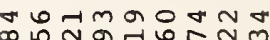

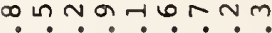
$\therefore \dot{0} \dot{0} \dot{\sim} \dot{0} \dot{0} \dot{0}$ न

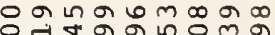
iिं- $\dot{\sim} \dot{m} \dot{m}$

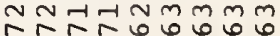

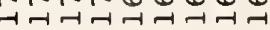

Nำ $\begin{array}{ccccccc}1 & 1 & 1 & 1 & 1 & 1 & 1 \\ -1 & -1 & -1 & -1 & -1 & -1\end{array}$

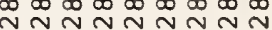
ก ก ก ก ก ก OOO 000000

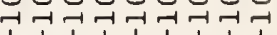

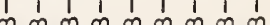

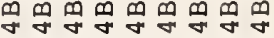
$\begin{array}{lllllllll}1 & 1 & 1 & 1 & 1 & 1 & 1 & 1 & 1\end{array}$

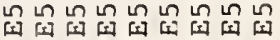

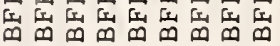




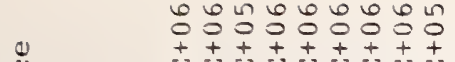

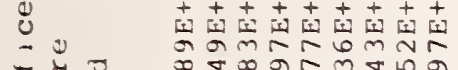
= प्रु

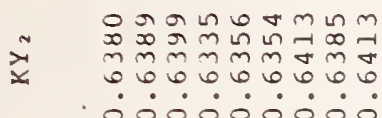

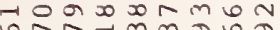
6. ○ं $\dot{0} \dot{0} \dot{0} \dot{0}$

ช Rูก

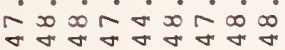

थ 3000

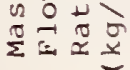

क

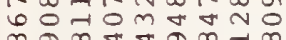
$\infty \sigma \infty \sigma \sigma \infty r \infty$ -ंல்-ंن்

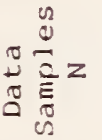

a

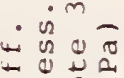

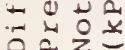

- Non

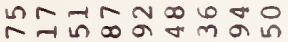
$\dot{m} \dot{\dot{n}} \dot{\sim} \dot{\sim} \dot{m} \dot{\sim}$ ₹

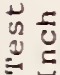

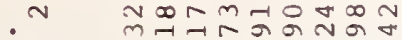

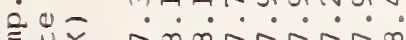

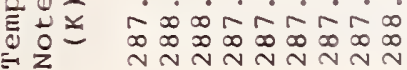

$\therefore$

¿

in nN

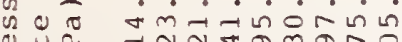
Uั

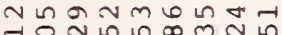

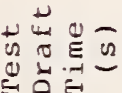

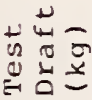

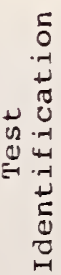

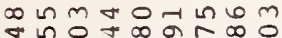
$\therefore \dot{a}-\dot{0} \dot{0}$

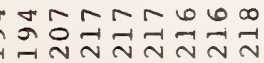

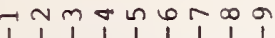
น่ํำ

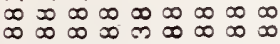

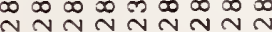
O O O O O O O O O $7 \rightarrow-7 \rightarrow 7-71$

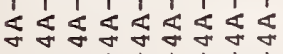

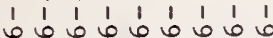

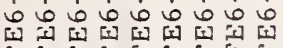

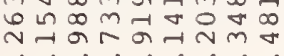
$\dot{\sim} \ddot{\circ}-\dot{\sim} \dot{\sim} \dot{\sim} \dot{0}$ ○ं $\dot{0} \dot{0} \dot{0} \dot{0}$ $6 \infty \dot{0} \dot{0} \dot{0}$ í $\dot{0} \dot{\sim} \dot{0} \dot{-1} \dot{0}$

ㅇํำำ

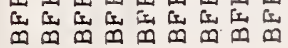

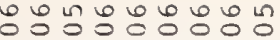

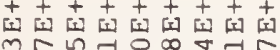

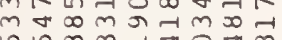

กำ

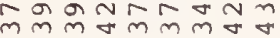

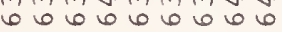
000000000 约

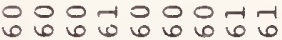

An $\rightarrow n \infty N$ n

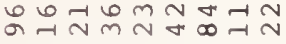

ก $-1 \backsim m \infty-16+$ 元舟

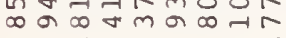

N

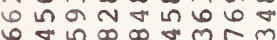
0 L $\infty \infty \forall m$ miñmm

ô $\dot{\circ} \dot{0} \dot{0} \dot{0} \dot{0} \dot{0}$ ำ ำ ำ ำ ำ

randomnL ம் $\dot{\sim} \dot{0} \dot{\sim} \dot{\sim} \dot{\sim}$

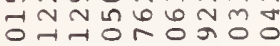

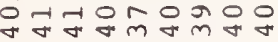
ํำ ถูก in $\dot{0} \dot{0} \dot{0} \dot{0}$ -

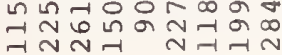

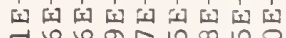
$\infty$ or $\infty$

m $m$ क

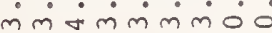

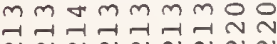

INM

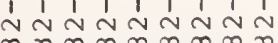

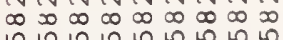
in กNNNNNำ $\begin{array}{lllllllll}1 & 1 & 1 & 1 & 1 & 1 & 1 & 1 & 1\end{array}$

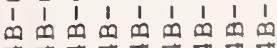

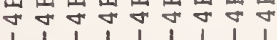
0000000000

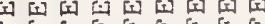

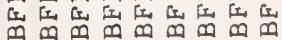

웅늉요유유요 $+t+t+t+$ $\exists$ G Jin m $m$ m vi $\dot{\sigma} \dot{A} \dot{i} \dot{\sim} \dot{\sigma}$

- J Jom 00

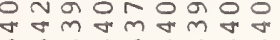

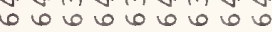
000000000

An.

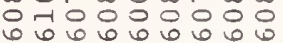
-00000000

a $\infty m \varphi$ año N N

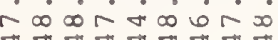

NNGUR LNO ㄱำ

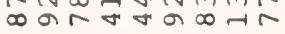
-िं-i்

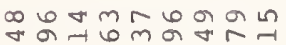

Dom กำ mंx

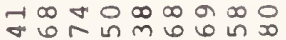

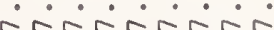
$\stackrel{\infty}{\infty} \stackrel{\infty}{\sim} \stackrel{\infty}{\sim} \stackrel{\infty}{\sim} \stackrel{\infty}{\sim} \stackrel{\infty}{\sim} \stackrel{\infty}{\sim}$

moman. - $\dot{0} \dot{0} \dot{0} \dot{0} \dot{0}$

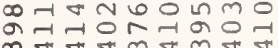

约 $-\therefore \dot{0} \dot{0} \dot{0}$ Norin ㅇำㅇำ

은 ? ? ? กัก N N ननकनानक

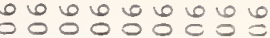
[x] [x] $06-\infty 6-190$ Nm $6 \infty \sim 00 \%$ 约원

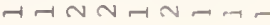

"थm

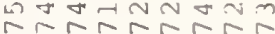
ด6 6606660 000000000

Mํ에

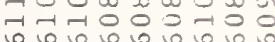
000000000

응ํำ mmu बै का m.

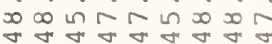

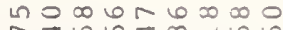

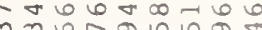
भ่

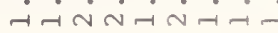

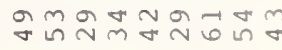

NMGDNRON

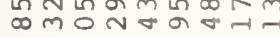
m $m \sim \infty \sim N m$ in

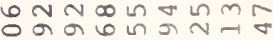
$\infty r \dot{0}-i \infty \infty$

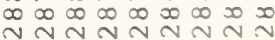

nhoomnto aniómm-

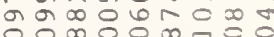
웜의의

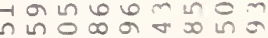

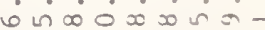
$\neg \underset{\sim}{\sim} \infty \infty \cdots$

$\infty m n-\pi) \pi$ पा

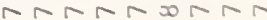

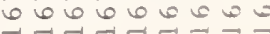

- $4 m \pi m b a \infty a$ ง $\infty \infty \infty \infty \infty \infty \infty \infty \infty$ 경ㄷㅇㅇㅇㅇㅇㅇㅇㅇㅇㅇㅇㅇㅇㅇㅇㅇ 1

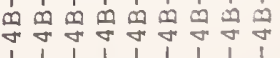
$060 \varphi v 10 \varphi 0$

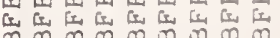

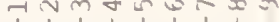

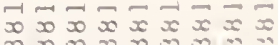
000000000

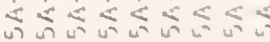

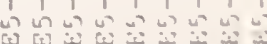

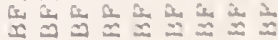
๒ือง 


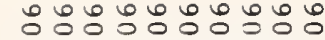

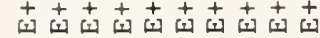
- 0 0 $\rightarrow$ प मे

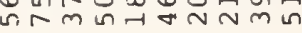

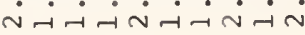

$0 m n \infty \pi \omega m \infty n$ 융ำ

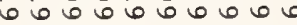
ல0ं0்0000

Uम मोल

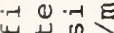

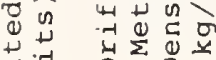

$\stackrel{-1}{-1}$

$\stackrel{0}{\mathscr{U}}$

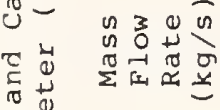

$\overbrace{0}^{2}$

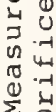

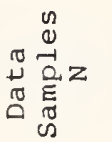

$i^{m}$ แथ थे क त 开 0

ڤै

in

E.

$\therefore$

둥

U

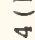

$\frac{0}{20}$

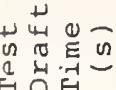

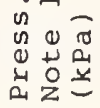

욜

の

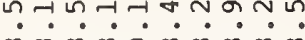
$\infty \infty \infty \infty \infty \infty \infty \infty \infty$ $\stackrel{\infty}{\sim} \stackrel{\infty}{\sim} \stackrel{\infty}{\sim} \stackrel{\infty}{\sim} \stackrel{\infty}{\sim} \stackrel{\infty}{\sim} \stackrel{\infty}{\sim} \stackrel{\infty}{\sim} \stackrel{\infty}{\sim}$

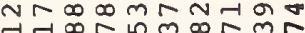

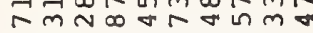
$\ddot{H} \dot{\sim} \dot{m} \dot{m} \dot{m} \dot{\sim} \dot{m} \dot{H}$

两 $\dot{N} \dot{\infty} \dot{\infty} \dot{\sim} \dot{\sim} \dot{\sim} \dot{\sigma} \dot{m}$

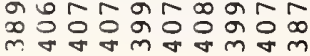

ต * $\infty \pi n$ In

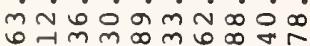
ت-1 $m_{-1}^{\infty} \infty n_{-1}^{\infty}$

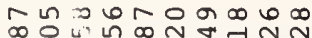
is 너든 $+\infty$ \%

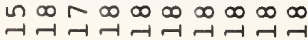

- $N m+n \in N \infty$ o

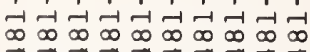

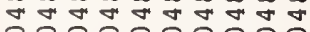
000000000 ڤ

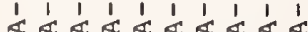

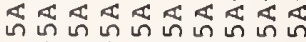

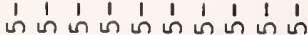

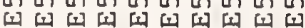

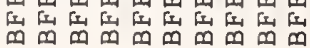

ஜ๐๐๐ㅇ

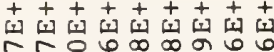
ก๊。

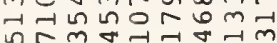

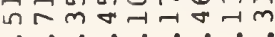
$\dot{v-i} \dot{i}-\dot{i} \overrightarrow{-}$

人 क ष० का जी $6 \circ$

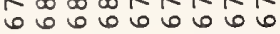
○் $\dot{0} \dot{0} \dot{0} \dot{0}$

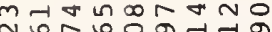
ㄴ. 00 क 다 ப் $\dot{0} 0 \dot{0} 0 \dot{0}$

$\operatorname{man} \pi$ N ก $\dot{0} \dot{0} \dot{0} \dot{0} \dot{0} \dot{0}$

$\forall \backsim \infty m \infty m m \infty$ กษ ป $\dot{\infty} \dot{\infty} \dot{0} \dot{\sim} \dot{\sim} \dot{\sim} \dot{\sim} \dot{\sigma}$

न

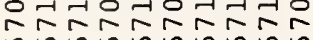
ด 6 ด 6 ด - $\dot{0} \dot{0} \dot{0} \dot{0} \dot{0} 0$

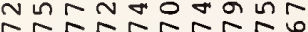
0000000000 6 6066610666 ن 0 ن $0 \dot{0} \dot{0} \dot{0} 0$

$\infty \pi+\pi / m$ Nํํ

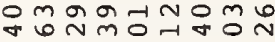
$\dot{\sim} \dot{-} \dot{\sim} \dot{\sim} \dot{\sim} \dot{\sim}$

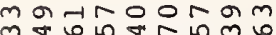

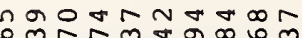
ด ก Nก $\dot{-i} \dot{\sim} \dot{-} \dot{\sim} \dot{-} \dot{\sim} \dot{N}$

용ㅇㅇำ

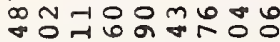
$\dot{H} \dot{n} \dot{m} \dot{\sim} \dot{m} \dot{m}$

ช i் $\dot{0} \dot{0} \dot{0} \dot{0} \dot{0}$

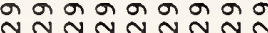

\#OM $\dot{0} \dot{0} \dot{0} \dot{0} \dot{0} \dot{0}$

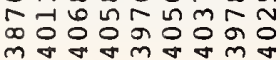

क त $\dot{\sim} \dot{m} \dot{m} \dot{\sim} \dot{\sigma} \dot{\sim}$

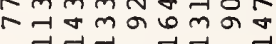

금ำ $N m \sim N$

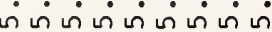
$\infty \infty \infty \infty \infty \infty \infty \infty \infty$ $\rightarrow-1-\infty \infty \infty$

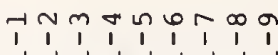

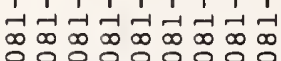
ก ก N O $0000000 \%$

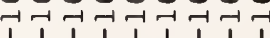
吅品品品品

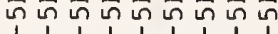

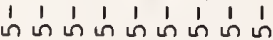

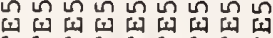

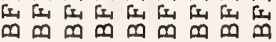

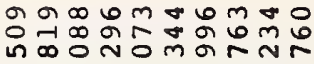
$\dot{\sim} \dot{\sim} \dot{\mu} \dot{m} \dot{\infty} \dot{m} \dot{\sim} \dot{-1}$

№내ำ . narinnaríb

MNO $\dot{m} \dot{0} \dot{0} \dot{0} \dot{0} \dot{0} \dot{-} \dot{0}$

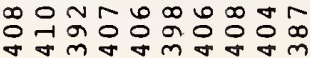

ป ปกำ N $\dot{\sim} \dot{m} \dot{0} \dot{0} \dot{0} \dot{\sim}-1$

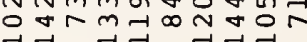

aำ $\dot{\sigma} \dot{\dot{\theta}} \dot{0} \dot{\sigma} \dot{\sigma} \dot{\sigma} \dot{\sigma} \dot{r}$ arkinarara न- $\rightarrow-1 \rightarrow-1$

$\begin{array}{llllllll}1 & 1 & 1 & 1 & 1 & 1 & 1 & 1\end{array}$

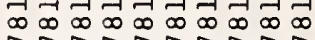

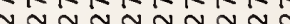

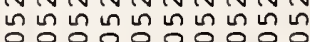
1
0 ง $\begin{array}{lllllllllll}1 & 1 & 1 & 1 & 1 & 1 & 1 & 1 & 1 & 1\end{array}$ ज曰

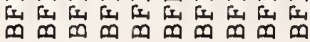

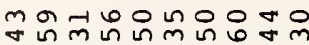

ஜ요요유요 $+++++++t+t+$

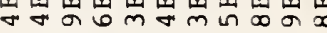

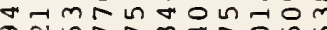

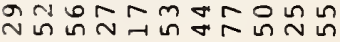
$\dot{-\dot{N}} \dot{\sim} \dot{\sim} \dot{\sim} \dot{-} \dot{-} \dot{-}$

4ก n. 6 606060606 ن் $\dot{0} \dot{0} \dot{0} \dot{0} \dot{0} 0$

I

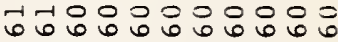
ن

น

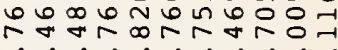

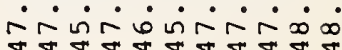

-1 न $m N m$ N 1 N N

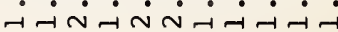

กำ

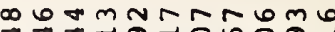

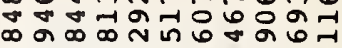
$\dot{\sim} \dot{m} \dot{\sim} \dot{\sim} \dot{\vec{\mu}} \dot{m} \dot{m} \dot{\sim} \dot{0}$ $\infty \infty \infty \infty \infty \infty \infty \infty \infty \infty$

aกNั - ? ? ? ? $\infty \infty \infty \infty \infty \infty+\infty$

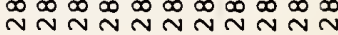

mantoGOONNir $\dot{0} \dot{0} \dot{0} \dot{0} \dot{0} \dot{0}$ in ำ

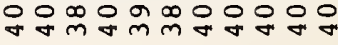

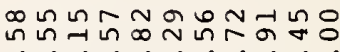

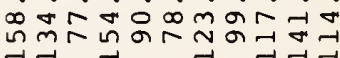

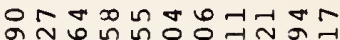
$\dot{a} \dot{0} \dot{0} \dot{0} \dot{0} \dot{\infty} \dot{\infty}$ ब

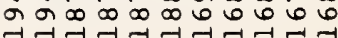
HNm
$-1-1+1-1-1-1-1-1$

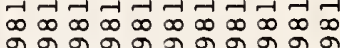

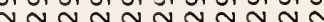

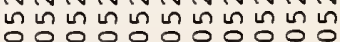

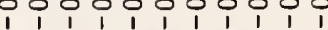
ผ $\begin{array}{lllllllllll}1 & 1 & 1 & 1 & 1 & 1 & 1 & 1 & 1 & 1 & 1 \\ 0 & 0 & 0 & 0 & 0 & 0 & 0 & 0 & 0 & 0 & 1\end{array}$ ज曰

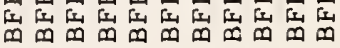


மீல் $+t+t+t+t+$

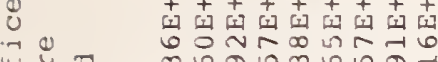
ঊ की

בับ

(I) خิ $\rightarrow 0 \cdot \pi$

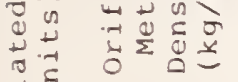

R N nOR n

$-\operatorname{i\sim n} \sim \dot{\sim}-\dot{-}$

กำ

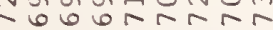
ต 60666660 000000000

G x 6 แn แก下

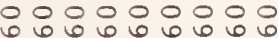
00000000

ด ᄂ

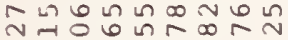
N -

0

\section{in 300 \\ एवृ $\approx$

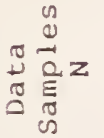

$\therefore$ in $\dot{\theta}^{m}=$

$=0$

돈욜

2

is

is

$\therefore \sigma$

:

s

$=$

$\frac{0}{2}$

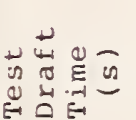

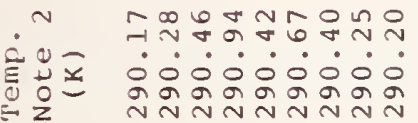

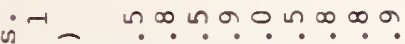

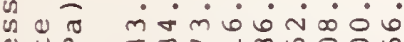

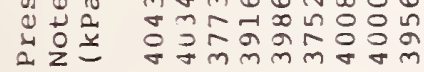

D

. ำ

एम

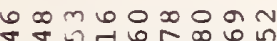
- $-\circ \circ \circ \circ$ Eᄂ

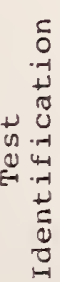

- $1 m$ \% ง $\infty \infty \infty \infty \infty \infty \infty \infty \infty$

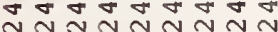
ก N N N N N N OOOOOOOOOO

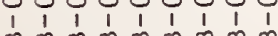

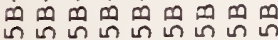
ก

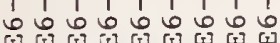

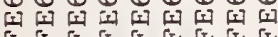

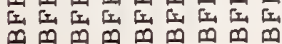

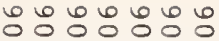
$t+t+t+t$ जूनूल

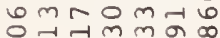
nm $\dot{-i} \dot{\sim} \dot{\sim} \dot{\sim}$

nNT

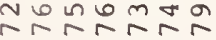
6 66606 ○ं0்0ல்

مَ-1 $6 \infty$

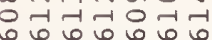
○ं0ं0் $\infty$
$\infty$
$\infty$
$\infty$
0

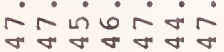

mom $m$ in กี่ भป⿻ - $\dot{\sim} \dot{\sim} \dot{\sim}$

กิำ 암을

$m m \infty \infty \omega n$

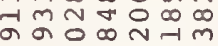
mंن்ini

นดm - 0.0 drorán

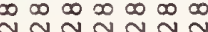

बa $\infty \sim \pi \ln$ $\infty \dot{\theta} \dot{0} \dot{0} \dot{r}$

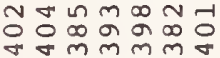

$\infty 0$ 임

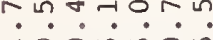

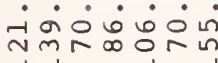

ㅍm $\therefore \dot{0} \dot{0} \dot{0} \dot{0}$

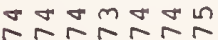

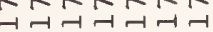

-1 2 m ป่⿻

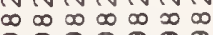
की

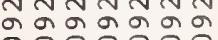
$\begin{array}{llllll}1 & 1 & 1 & 1 & 1 & 1\end{array}$ ต $\begin{array}{lllllll}0 & 0 & 0 & 0 & 0 & 0\end{array}$

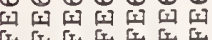

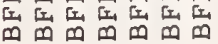

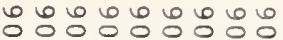
$t+t+t+t+t$ $\infty$ 그의

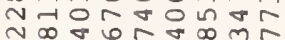
ก้ด ํำ

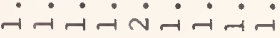

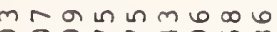

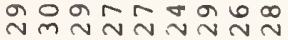
nminN ல் $\dot{0} \dot{0} 0 \dot{0}$

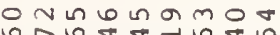
ongha

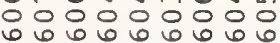
○ं 000000

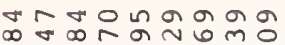
$\rightarrow-\sigma$ in in $\infty$ नू न $-60 \div 606$

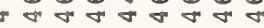

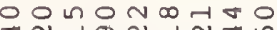
ก 0 \% -iि-i山r-ic

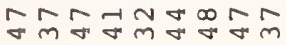

mNGONNOAN

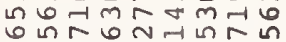

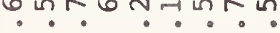
$\dot{\sim} \dot{\sim} \dot{m} \dot{m} \dot{N} \dot{v}$

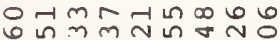
$\dot{0} \dot{0} \cdot \dot{0} \dot{0}$ 응ㅇㅇㅇㅇㅇㅢ 징ㅇㅇㅇㅇㅇㅇ

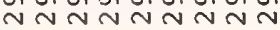

mmоm⿻ $\dot{\sim} \dot{\sim} \dot{\omega} \dot{\sigma} \dot{-} \dot{-} \dot{\omega}$

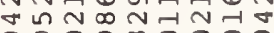

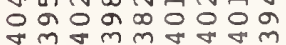

$m \infty m \backsim N \infty m \omega$ N $100 m-1$

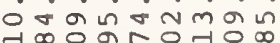
- $\infty 0$ aरण

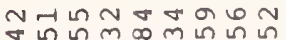
$\dot{m} \dot{m} \dot{m} \dot{m} \dot{m} \dot{m} \dot{m}$ $m m m m m m m$

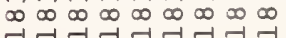

1)

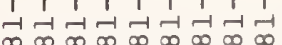
禹 ○) ? : 00 $\infty 0 \infty \infty$

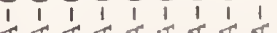

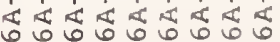
$\begin{array}{lllllllll}1 & 1 & 1 & 1 & 1 & 1 & 1 & 1 & 1\end{array}$

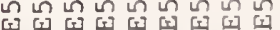

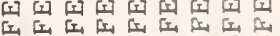

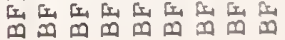

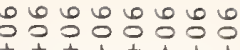

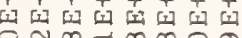
네

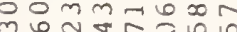

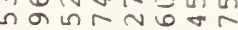

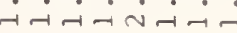

0 Nom $-\infty \circ \pi$

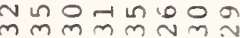
mañN 00000000

a $\rightarrow \infty m \backsim \rightarrow \infty-1$

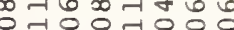
06006060 0000000

กㄴํำ유ำ

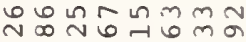
约

ImannoOn

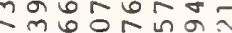

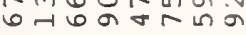
- $n-1+r-1$

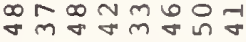

ommanna

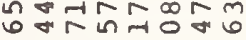
$\dot{\sim} \dot{\sim} \dot{\sim} \dot{0} \dot{\mathrm{m}}$

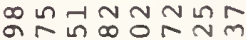
बㅇनननननन กำกำ

anbronn $\therefore \dot{0} \dot{0} \dot{0} \dot{0}$ กलm N N

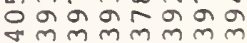

จำ-

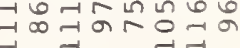

๗ㄷำ $\dot{0}$ ก่

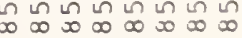

$\rightarrow-1-1-1 \vec{x}-\vec{x}$ ○ 0 0 0000

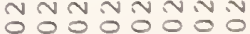
1. 吕出 00000

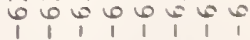

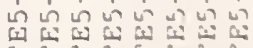

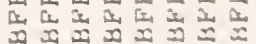




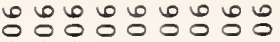
U) -10 (1)

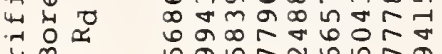

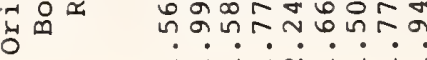

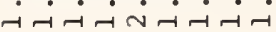

aกนก

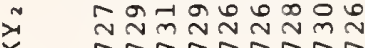

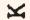

-00000000

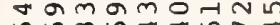

^. 606060606 ○ं000000

ปै प्रेल 开

$\sim \infty m m \infty \circ \sim \infty$ moन

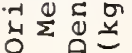

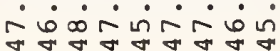

皆包

兄

这占

$\prod_{0}^{\infty} \frac{1}{c}$

$\therefore$

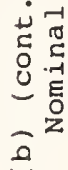

อ

है
น थ०

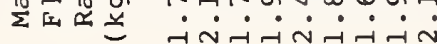
告

- is is ${ }_{i}^{m}-$ $\omega_{-1}^{\infty} \omega_{0}$ 武造道

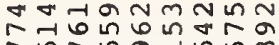

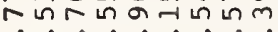
$\dot{\sim} \dot{\sim} \dot{\sim} \dot{m} \dot{\sim} \dot{\sim} \dot{\sigma}$

\section{.}

是 $\overline{0}$ U.

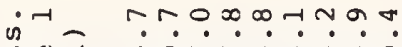

uै ब

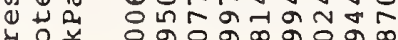

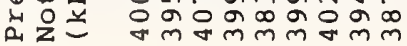

or

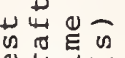

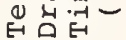

on

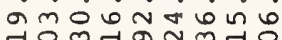

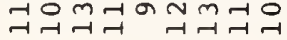

음요으ำำ

幽若 क

$\dot{\sim} \dot{m} \dot{m} \dot{\sim} \dot{m} \dot{\sim}$

(i) 넌도

ำกำกำกำ

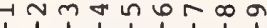
ง

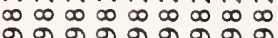
กง ข 000000000 1

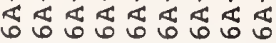
0

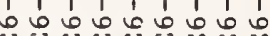

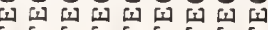

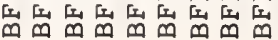

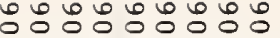

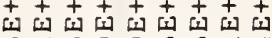
6 ปી *

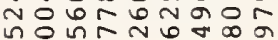

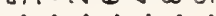

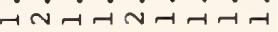

nodhan an

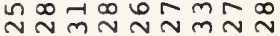
vinknkvin ○ं $\dot{0} \dot{0} \dot{0} \dot{0} \dot{0}$

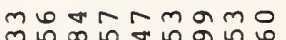

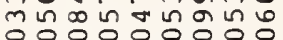

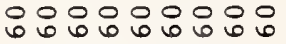
○ं $\dot{0} \dot{0} \dot{0} \dot{0} 0$

욤ำ ㅇำถ์

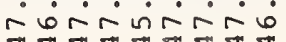

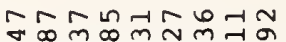

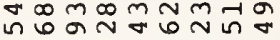

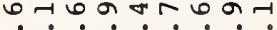
$\dot{\sim} \dot{\sim} \dot{-} \dot{\sim}-\dot{-1} \dot{N}$

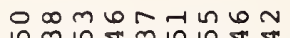

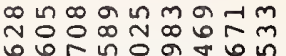
b 0 r vं $\dot{\sim} \dot{m} \dot{\sim} \dot{\sim} \dot{\sigma}$

6 o $\infty$ Nom 0 n. ririrrinio $\infty \infty \infty \infty \infty \infty \infty \infty \infty$

a in tomog n

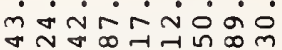

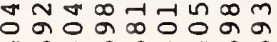
$m \propto m m$

ก 익 का

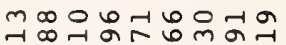
- $\dot{\sim} \dot{\sim} \dot{\sim} \dot{\sim} \dot{N}$

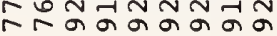
ती

HNm ง.n.

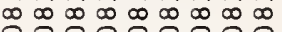

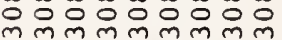
नूनूनून 0 o 0 인

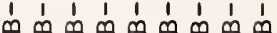

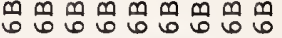
$\begin{array}{lllllllll}1 & 1 & 1 & 1 & 1 & 1 & 1 & 1 & 1 \\ 0 & 0 & 0 & 0 & 0 & 0 & 0 & 0 & 0\end{array}$

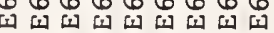

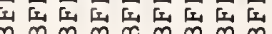

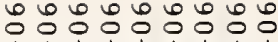

$++t+t+t+t$

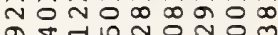
厅ㅁำ

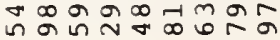
$\dot{-\dot{\sim}} \dot{\sim} \dot{\sim} \dot{-} \dot{-} \dot{-}$

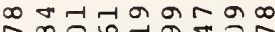

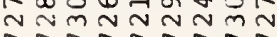
hanknthra ०0ं0் 000

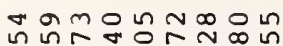

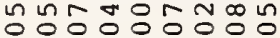

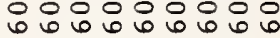
000000000

m 0 N

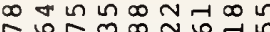
. $0.0 \cdot 0 \cdot 0$

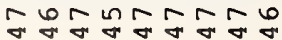

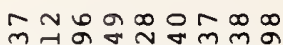
कै जี

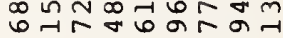

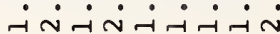

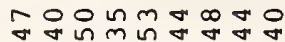

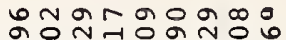

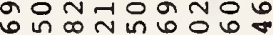
$\dot{N} \dot{\sim} \dot{\sim} \dot{\sim m} \dot{m} \dot{\sigma}$

nmbraOn a (n) $\infty r \dot{r} \dot{\infty} \dot{\infty} \dot{\infty}$ $\stackrel{\infty}{\sim} \infty \infty \infty \infty \infty \infty \infty \infty \infty$

$\infty \infty \infty r-a m \infty a$

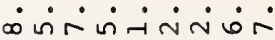
in in inarodod

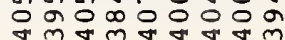

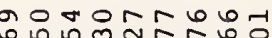
- $\dot{0} \dot{0} \dot{0} \dot{0} \dot{0}$ o कor

ฟ้ㅇㅇำ نं $\dot{1} \dot{\alpha} \dot{\alpha} \dot{\alpha} \dot{\alpha}$ $\tau \infty \infty \infty \infty \infty \infty \infty \infty \infty$

-1 Nm n

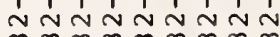
$\infty \infty \infty \infty \infty \infty \infty \infty \infty$ ㅇㅇㅇㅇㅇㅇㅇㅇㅇㅇㅇㅇㅇ

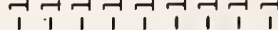

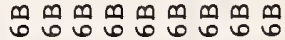
Q

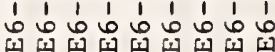

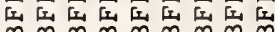

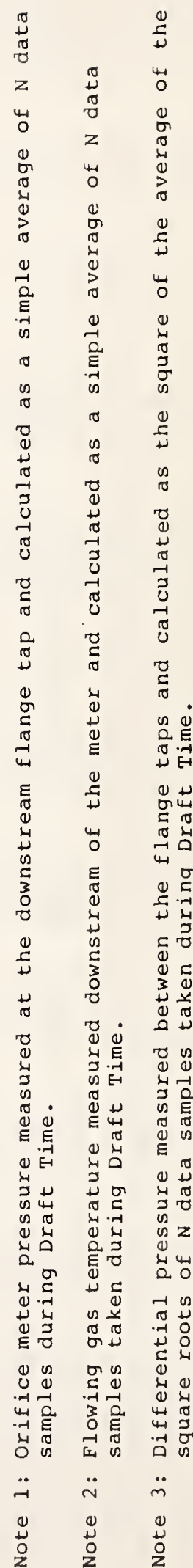


Table 5(a). Test Meter Identification and Physical Measurements Nominal 6 Inch AGA/API Orifice Meter (SI Units).

$\begin{array}{ccccc}\text { Test } & \text { Test } & \text { Run } & \text { Orifice } & \text { Beta } \\ \text { Identification } & \text { Configuration } & \text { Diameter } & \text { Diameter } & \text { Ratio } \\ & & (\mathrm{cm}) & (\mathrm{cm}) & \end{array}$

\begin{tabular}{|c|c|c|c|c|}
\hline $\begin{array}{l}\text { BFE7-1A-020382-[10] } \\
\text { BFE7-1B-020582-[9] } \\
\text { BFE8-1A-012982-[8] } \\
B F E 8-1 B-051282-[12] \\
B F E 7-2 A-020282-[9]\end{array}$ & $\begin{array}{l}1982-\mathrm{B} \\
1982-\mathrm{B} \\
1982-\mathrm{B} \\
1982-\mathrm{B} \\
1982-\mathrm{B}\end{array}$ & $\begin{array}{l}15.4173 \\
15.4173 \\
15.4160 \\
15.4160 \\
15.4173\end{array}$ & $\begin{array}{l}3.1725 \\
3.1727 \\
3.1725 \\
3.1727 \\
5.7147\end{array}$ & $\begin{array}{l}0.2058 \\
0.2058 \\
0.2058 \\
0.2058 \\
0.3707\end{array}$ \\
\hline $\begin{array}{l}\text { BFE7-2A-021182-[9] } \\
\text { BFE7-2B-020982-[10] } \\
\text { BFE7-2B-021082-[9] } \\
\text { BFE8-2A-012782-[9] } \\
\text { BFE7-3A-020482-[9] }\end{array}$ & $\begin{array}{l}1982-B \\
1982-B \\
1982-B \\
1982-B \\
1982-B\end{array}$ & $\begin{array}{l}15.4173 \\
15.4173 \\
15.4173 \\
15.4160 \\
15.4173\end{array}$ & $\begin{array}{l}5.7147 \\
5.7150 \\
5.7150 \\
5.7147 \\
7.6208\end{array}$ & $\begin{array}{l}0.3707 \\
0.3707 \\
0.3707 \\
0.3707 \\
0.4943\end{array}$ \\
\hline $\begin{array}{l}\text { BFE7-3A-021182-[9] } \\
\text { BFE7-3B-020382-[9] } \\
\text { BFE8-3A-051382-[12] } \\
\text { BFE8-3B-012782-[9] } \\
\text { BFE7-4A-020982-[9] }\end{array}$ & $\begin{array}{l}1982-B \\
1982-B \\
1982-B \\
1982-B \\
1982-B\end{array}$ & $\begin{array}{l}15.4173 \\
15.4173 \\
15.4160 \\
15.4160 \\
15.4173\end{array}$ & $\begin{array}{l}7.6208 \\
7.6210 \\
7.6208 \\
7.6210 \\
8.8885\end{array}$ & $\begin{array}{l}0.4943 \\
0.4943 \\
0.4943 \\
0.4944 \\
0.5765\end{array}$ \\
\hline $\begin{array}{l}\mathrm{BFE} 7-4 \mathrm{~B}-020482-[9] \\
\mathrm{BFE} 8-4 \mathrm{~A}-051382-[12] \\
\mathrm{BFE} 8-4 \mathrm{~B}-012982-[9]\end{array}$ & $\begin{array}{l}1982-B \\
1982-B \\
1982-B\end{array}$ & $\begin{array}{l}15.4173 \\
15.4160 \\
15.4160\end{array}$ & $\begin{array}{l}8.8882 \\
8.8885 \\
8.8882\end{array}$ & $\begin{array}{l}0.5765 \\
0.5766 \\
0.5766\end{array}$ \\
\hline
\end{tabular}

Note: Test Identification bracket gives number of tests run with this geometry. A total of 172 tests were run on the nominal 6 inch run size. 
(200:

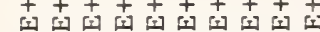
$\rightarrow$ N $\rightarrow$ N फ का का $m m \infty N m$ in $N$ แn 0 N

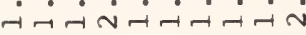
$\rightarrow$ व. -1 पू पु $\Sigma$ Q

$\underset{m}{\sim}$ 의 $\dot{m}$ 4 is 0 is 出过荝 대믈

造

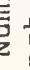

$\forall 6 \sim m-n \sim m \sim m$

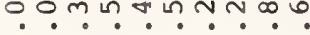
istrantar $\stackrel{\infty}{\sim} \sim \mathrm{v}^{\infty} \stackrel{\infty}{\sim} \stackrel{\infty}{\sim} \stackrel{\infty}{\sim} \stackrel{\infty}{\sim} \stackrel{\infty}{\sim} \stackrel{\infty}{\sim} \stackrel{\infty}{\sim}$ nan motnio n NA $=$ -

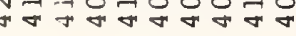

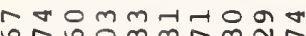

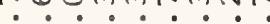

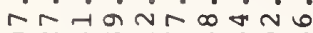
onm $\sim \pi m \infty 0 \infty$

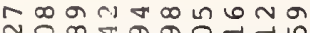
40

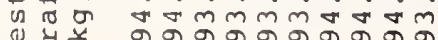

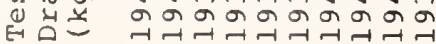

HNm

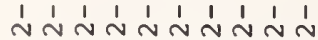

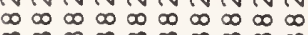
o o o o o o o의

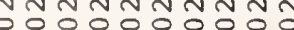
$\begin{array}{lll}1 & 1 & 1\end{array}$

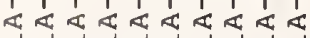

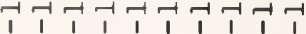

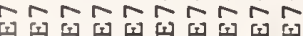

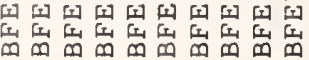

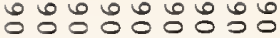
$+++++++++$

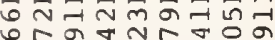
담 ঢี -i-irina-i

$m m \amalg m \in$ ก กำกำ

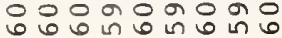
¿0ं 0ல0 000

$\infty \infty 0 \infty$ 구욤ㅇ

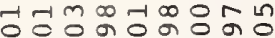
๑ 00 in 0 in 0 in 6 0ं0் 0000

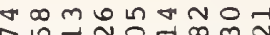

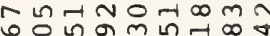

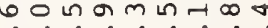

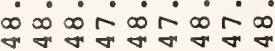

n $m \rightarrow n \circ$ on m으는ำำกำ M⿻上丨 ○ं0

엄 ๓ $\dot{\sim} \dot{m} \dot{\sim} \dot{\infty} \dot{\infty} \dot{\sim} \dot{\sim} \dot{m} \overrightarrow{-}$

음음ำ ㄱ. 으으.

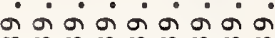
$\stackrel{\infty}{\sim} \stackrel{\infty}{\sim} \stackrel{\infty}{\sim} \stackrel{\infty}{\sim} \sim \infty \underset{\sim}{\infty} \stackrel{\infty}{\sim} \sim \stackrel{\infty}{\sim} \stackrel{\infty}{\sim} \stackrel{\infty}{\sim}$

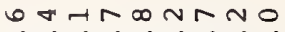
$\dot{\sim} \dot{\dot{m} \dot{m}} \dot{0} \dot{0}$ *

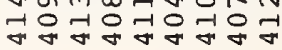

N $\infty \cup \cup m N \infty \backsim$ Im $000000 \infty$ نं $\dot{0} \dot{0} \dot{\sigma} \dot{i} \dot{N}$ nㅇㅇㅇㅇㅠ

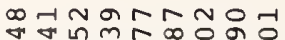
NNNN

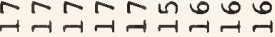

-

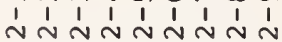

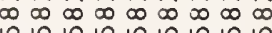

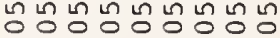

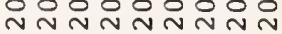
이잉

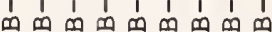

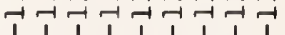

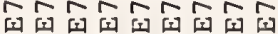

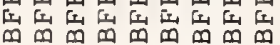

○ீ $++++++++$

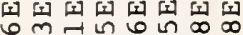

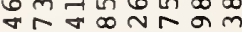
जि थून न $m \infty N \rightarrow m \sigma N$ แn $\dot{-i} \dot{\sim} \dot{-} \dot{-1}$

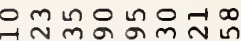
ส กิรูก ชำำ ํํำ ல0் 0000

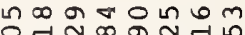
ㄱำ 응ㅇํ ல0் 00000

n $\backsim \infty \infty \infty-1 \infty$ mำ ?

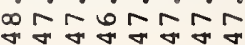

$\infty m \backsim \sim m \infty 0$ $m$ N ชิ ல0் $0 \dot{0} 0 \dot{0}$

$\infty-1 \infty \backsim \nabla \sigma \sigma$

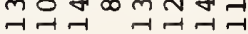

Hogmn 0 는 D

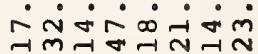

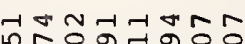
ITOOJGO $\dot{\infty} \dot{\infty} \dot{\infty} \dot{\sigma} \dot{\sigma} \sigma$ $\infty \infty \infty \infty \infty \infty \infty \infty$ $\stackrel{\infty}{\sim} \stackrel{\infty}{\sim} \stackrel{\infty}{\sim} \stackrel{\infty}{\sim} \stackrel{\infty}{\sim} \stackrel{\infty}{\sim} \stackrel{\infty}{\sim}$

0 แn ब $\sigma \ln \infty \pi$

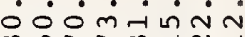
morm. 감의

$\infty \infty \nabla \forall \backsim \sim 0=$ $\because m-70 \forall m$ $\dot{0} \dot{0} \dot{\sim} \dot{0} \dot{\sim}$

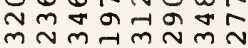

ดูกํำ

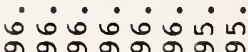

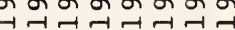

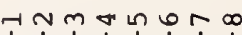

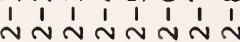

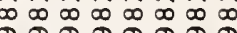
ลิำกำลำ ำ

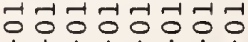
$\begin{array}{llllllllllllll}1 & 1 & 1 & 1 & 1 & 1 & 1 & 1\end{array}$

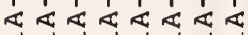

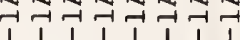

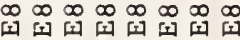

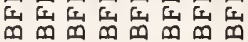

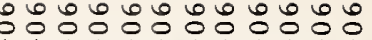

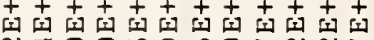

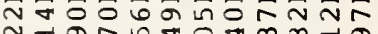

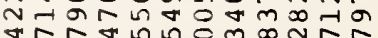
ํำ

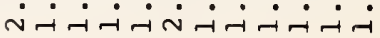

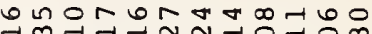

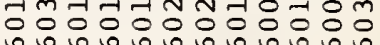

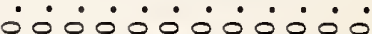

ㅇำ-

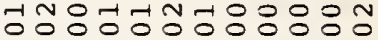
606060606060 ○ं $\dot{0} 0 \dot{0} 0 \dot{0} 0 \dot{0}$

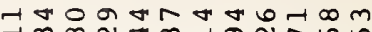

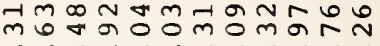

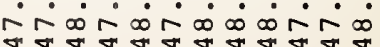

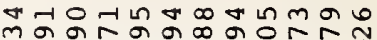

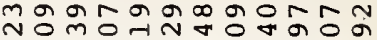
$0 \infty$ in - $0 \dot{0} \dot{0} \dot{0} \dot{0} \dot{0} \dot{0} 0$

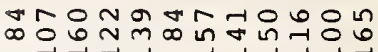

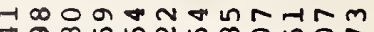
ด

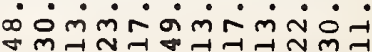

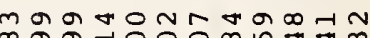

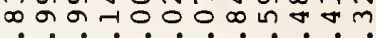

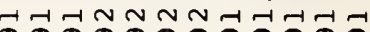

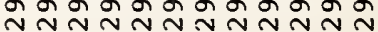

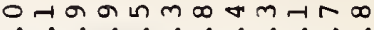
수워ํ

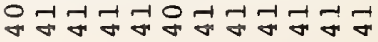

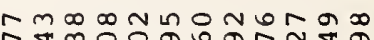
กตㅇำ

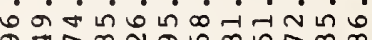

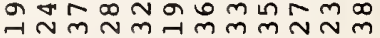

ㄲำกำㅇำ

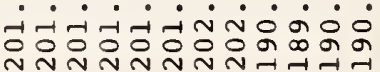

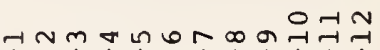

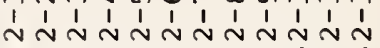
$\infty \stackrel{\infty}{\infty} \underset{\sim}{\infty} \stackrel{\infty}{\sim} \stackrel{\infty}{\sim} \stackrel{\infty}{\sim} \stackrel{\infty}{\sim} \stackrel{\infty}{\sim} \stackrel{\infty}{\sim} \stackrel{\infty}{\sim} \stackrel{\infty}{\sim} \stackrel{\infty}{\sim}$

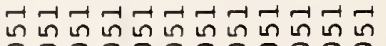

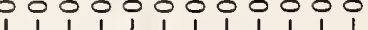

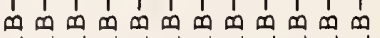
ન્રન

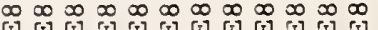

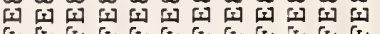

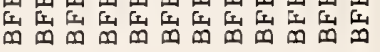




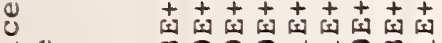
H. I ए

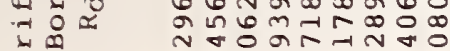
ơ

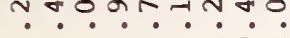
- $\dot{0}-\dot{N}-\dot{a}-\dot{ }$ $\ddot{\ddot{x}}$

సี

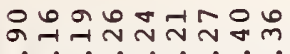

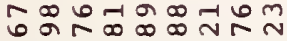

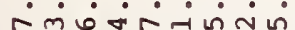

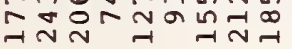

HNm n

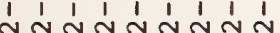

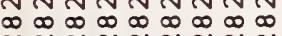
$\sim \sim \mathcal{N} N \sim \sim N N$

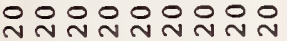
i $0^{1} 0_{1} 0_{1} 0_{1} 0_{1} 0_{1}$

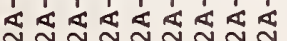
I I I I I I I I I $r r r r r r$

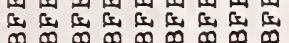

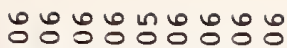

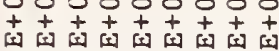

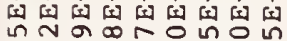

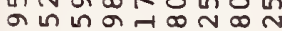
mo 0 แ $010 \mathrm{~m}$ - ON -Im $\rightarrow$ N $\operatorname{NN}$ N

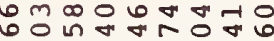

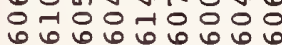
○ं $0 \dot{0} 0 \dot{0} 00$

m n $0 m \infty n \pi+m$

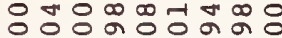

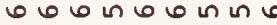
○ं 0000000

늠

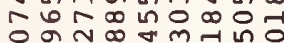
$\dot{\sigma} \dot{\sigma} \dot{0} \dot{0} \dot{\sigma} \dot{0} \mathbf{r}$

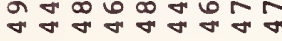

न1

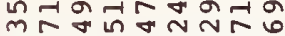

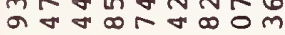
$\therefore \sim \dot{0} \dot{\sim} \dot{-}$

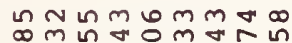

mกN $\frac{1}{4}$ o ด̊ำ min - $\dot{0} \dot{0} \dot{0} \dot{0}$ 웅ㅇㅇ엉응

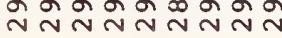

nutunama

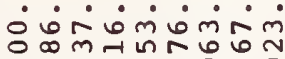

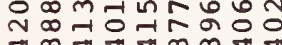

๒ ก $\dot{0} \dot{0} \dot{0} \dot{\infty} \dot{m} \dot{0}$ 낭ㅇำ 각ำ

유테mm

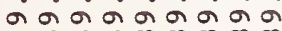
$\infty \infty \infty \infty \infty \infty \infty \infty \infty \infty$

HNm n

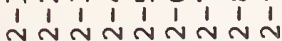
$\infty \infty \infty \infty \infty \infty \infty \infty$

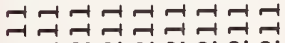
NNNNNN $\begin{array}{lllllllll}1 & 1 & 1 & 1 & 1 & 1 & 1 & 1 \\ 0 & 0 & 0 & 0\end{array}$

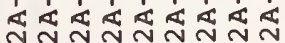
$\begin{array}{llllllll}1 & 1 & 1 & 1 & 1 & 1 & 1 & 1 \\ 1 & 1\end{array}$

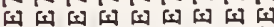

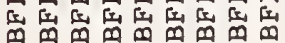

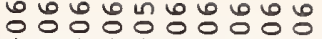

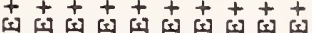

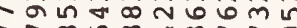

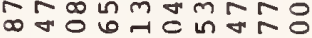
范윰ำ $\dot{-1} \dot{i} \dot{\sim} \dot{m} \dot{\sim} \dot{-i}$

m的

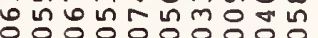
๑ 0600600060 - $0 \dot{0} \dot{0} 00000$

แก कm a

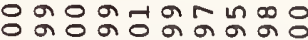

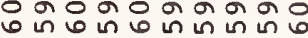
○ं0் $0 \dot{0} \dot{0} 0 \dot{0}$

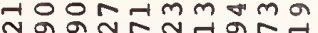
กิ $\dot{\sigma} \dot{\sigma} \dot{\omega} \dot{\infty} \dot{\sigma} \dot{\sigma} \dot{\infty} \dot{\sigma}$ $\forall \forall \xi \forall \forall \forall \forall \approx$

mกRn

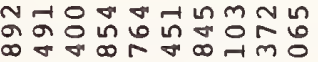
onh-ion-ing

ન่m

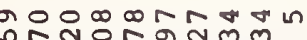

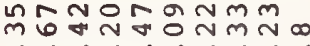
$\dot{m} \dot{\infty} \dot{\infty} \dot{\sim} \dot{\infty} \dot{\sim} \dot{\infty} \dot{\infty}$ $\sim \sim \sim \sim$

구ำm nm nu n - 00000000

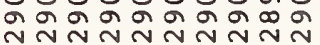

ก $\infty \approx$ ம

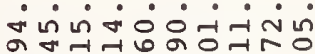

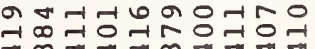
$\sigma m \sigma \forall m \neq \sigma \sigma$

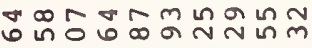
$\dot{m} \dot{0} \dot{0} \dot{0} \dot{\sim} \dot{\infty} \dot{\infty}$ 光

m n In

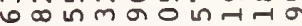

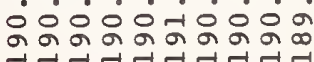

Tnm ง

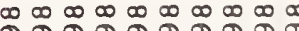

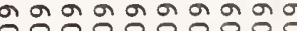

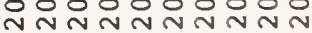
OOOOOOOOOO

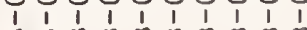
m $\begin{array}{llllllllll}1 & 1 & 1 & 1 & 1 & 1 & 1 & 1 & 1 & 1\end{array}$ ก)

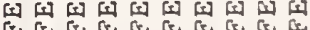

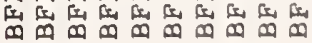

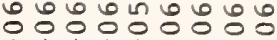
$+4+4+t+1$ अ ำ ก ธี่สํํㅇำกี - mं

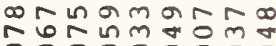

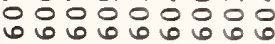
00000000

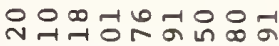

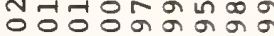
6006 in $\operatorname{con}$ in 000000000

$m \infty \omega \sigma \infty \infty N$ กั कां்ம் $\dot{0} \dot{0}$

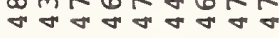

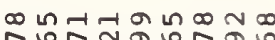
న.

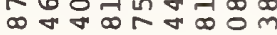
ONH HONHA-

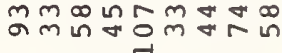

ดกMn日Han ஸैํำ $\dot{m} \infty \dot{\sim} \dot{\sim} \dot{\sim} \dot{\sim} \infty$

ชัษ $\dot{0} \dot{0} \dot{0} \dot{\circ} \dot{\circ}$ 음ㅇㅇㅇㅇ요영응

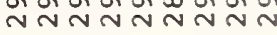

* - mo mㄷํㅇํำ

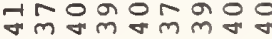

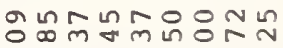
ம் $\dot{\sigma} \dot{\sigma} \dot{\sigma} \dot{\sigma} \dot{\sigma}$ aำ

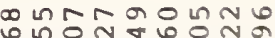
\% $\infty \infty^{\infty} \infty \infty_{\infty}^{\infty} \infty \infty_{\infty}^{\infty} \infty$

-1Nm งกาง $\infty \infty_{\infty}^{\infty} \infty \infty^{\infty} \infty \infty^{\infty} \infty$

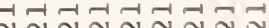
OOOOOOOO

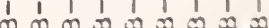

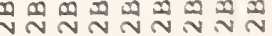
1 $11-1$

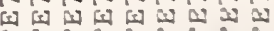

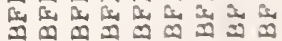




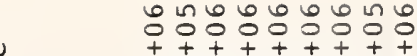
ชnNGน

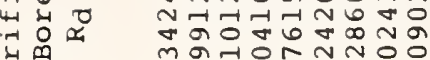
냉

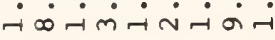

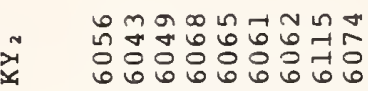
$\dot{0} \dot{0} \dot{0} \dot{0} \dot{0} \dot{0}$

番 年

$\stackrel{D}{0}_{0}$

$E$

$\therefore 0$

空

e

เ

党

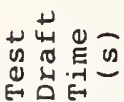

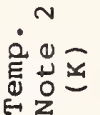

a $N M-1 N$ a ด जก

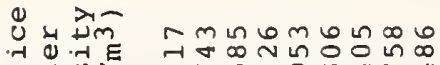

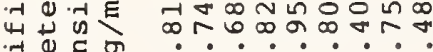

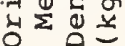

romang a

is 30 in

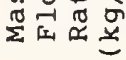

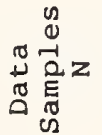

is in ${ }^{m}-$

出品落

向运

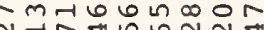
Nื ம் $\dot{\sim} \dot{\infty} \dot{\sigma} \dot{\sim} \dot{\sim}$

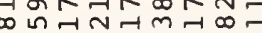
ำ

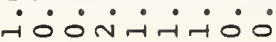

No์ नं $\sigma^{\circ}$ न $\sigma^{\circ} \sigma^{\circ}$

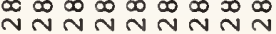

tmnamanta (1)

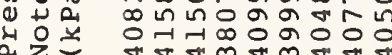
$\forall \forall m \forall m$

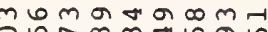
OाNDm

is क्ष Eᄂ $m \rightarrow \dot{\sim} \dot{\sim} \dot{0}$ क

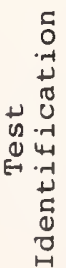

Tnman n.

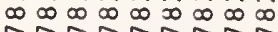

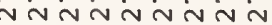

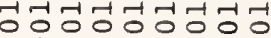

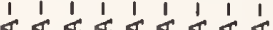

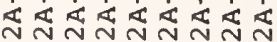
$\begin{array}{lllllllll}1 & 1 & 1 & 1 & 1 & 1 & 1 & 1 & 1 \\ 0 & 0 & 0 & 0 & 1 & 0 & 0 & 1 & 1\end{array}$

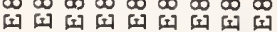

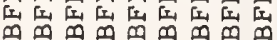

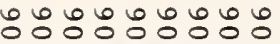
$++t+t+t+t$ a barno

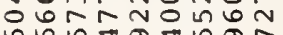
ใด้ Nmb No

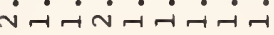

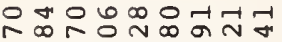

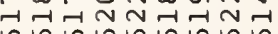
000000000

$m \pi m \infty$ a $m N$ n क 일 约 in 60 in 60 in - 0000000

mom $m-\infty n+6$ ㅇำ

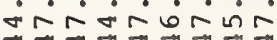

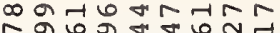
กิ่ง 용 $\dot{\sim} \dot{-} \dot{\sim} \dot{-} \dot{-} \dot{\sim} \dot{-}$

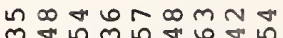

odn

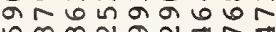
$\infty \dot{m} \dot{\infty} \dot{N} \dot{\sim} \dot{m}$

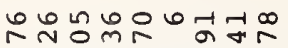
$\dot{0} \dot{\circ} \dot{0} \dot{0} \dot{\infty} \dot{\infty} \dot{0}$

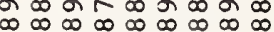

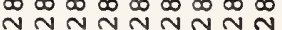

mn+oongn

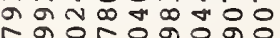

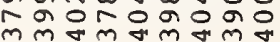

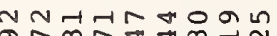
anm -i்

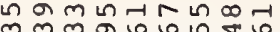
¿ㅇㅇㅇㅇㅇㅇㅇㅇ N N N N ㄴNㅇำ

$\rightarrow N m$ an ง่าง

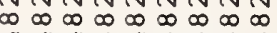

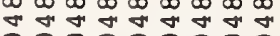
00000000 NNN NNN N N N o o o o o o o $\begin{array}{lllllllllll}1 & 1 & 1 & 1 & 1 & 1 & 1 & 1 & 1\end{array}$

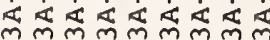

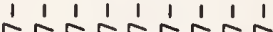

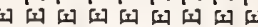

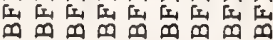

om 0 nom

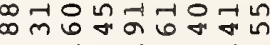
o 6 in

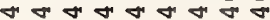

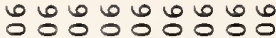

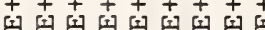
$\infty$ m $\infty \pi \infty \infty$

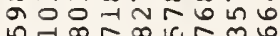
$\sim 6 \infty \approx-1, \infty \infty m$

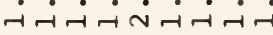

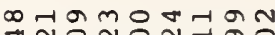
กNNNNㄱำ 6 66066066 000000000

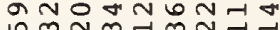

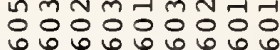
○ंல் 0ல்

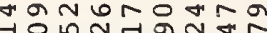

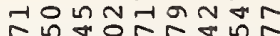

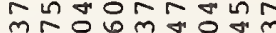

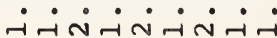

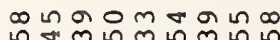

onanon mina 거ㄱㅡㅔ $\dot{\sim} \dot{\sim} \dot{m} \dot{\infty} \dot{\sim} \dot{\sim} \dot{\sim}$

บูก 4อ J iिंबं $\infty \infty \infty \infty \infty$

antornd th $\dot{0} \dot{0} \dot{0} \dot{0} \dot{0} \dot{0}$ a ज̂

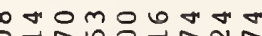
ontus n

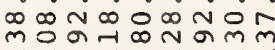

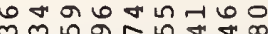
- $\dot{0} \cdot \dot{0} \cdot \dot{0}$

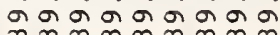
$\infty \infty^{\infty} \infty \infty \infty \infty \infty \infty$

INm-

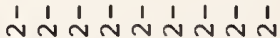
$\infty \infty \infty \infty \infty \infty \infty \infty \infty$

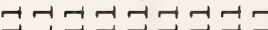

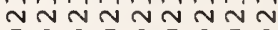
이잉ㅇㅇㅇ

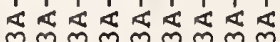
$\begin{array}{llllllll}1 & 1 & 1 & 1 & 1 & 1 & 1 & 1\end{array}$

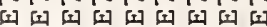

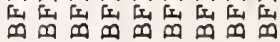

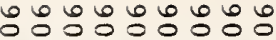

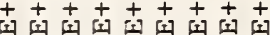
त) $\sim$ Nm omrmora a N N $6 \infty \pi-1 m \infty m \sim$

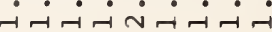

Nmona 0 내

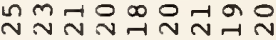
๑ 6606666 000000000

N

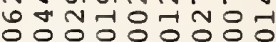
6 60606060 ○ं $0 \dot{0} \dot{0} 0 \dot{0}$

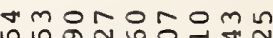
ก็ำ N660m6 60

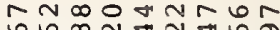

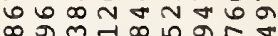
๓

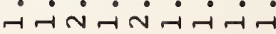

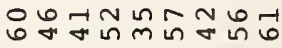

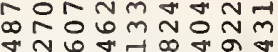
$\dot{N} \dot{\sim} \dot{m} \dot{\infty} \dot{\sim} \dot{\sim} \dot{N}$

$6 \infty$ 드

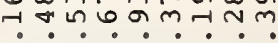
$\infty \infty \infty \infty \dot{\infty} \infty \dot{\infty} \infty \dot{\infty}$

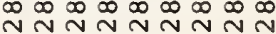

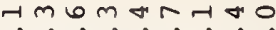
ம்in்rá mr익

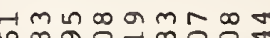
$6 \infty$ a

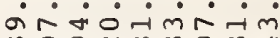
m०न त

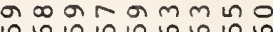
$\dot{m} \dot{m} \dot{m} \dot{m} \dot{m} \dot{m}$ बननकन नून

TNm ป่

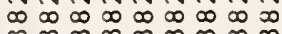

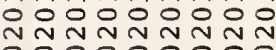
O 0 O O O O O O O

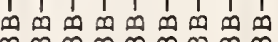
11111111

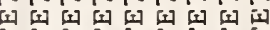

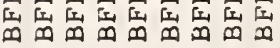




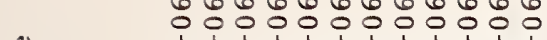

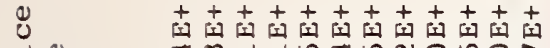

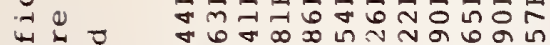
W $\omega_{0}^{\infty}$

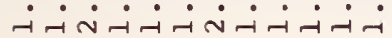

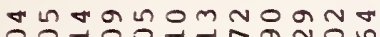

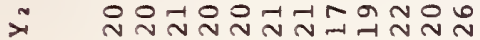

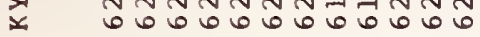
ல0000000000

nom nn - monnd ดิ กิ

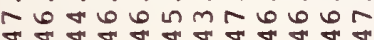

용요 m o의의

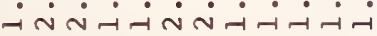

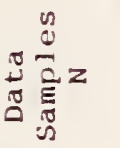
ㅇํㅇำ 茨

造 $=0$

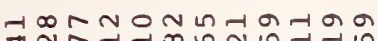

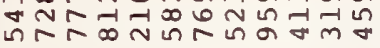
$\dot{m} \dot{\infty} \dot{\infty} \dot{\sim} \dot{\sim} \dot{N} \dot{N} \dot{m} \dot{\sim}$

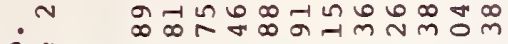
$\stackrel{0}{E}_{0}^{0} \underset{0}{0}$

$\therefore 0$

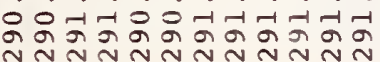

anommungand $\infty \sim \dot{\sim} \dot{\sim} \dot{0} \dot{0} \dot{0} \dot{0} \dot{0}$

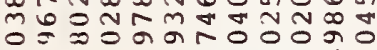

nUntan ก ก

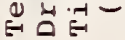

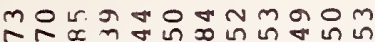
U

-1

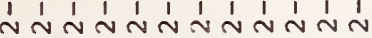

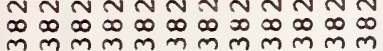

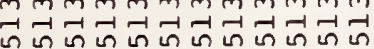

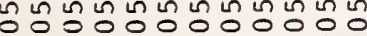

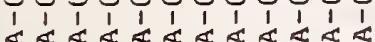

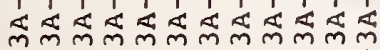

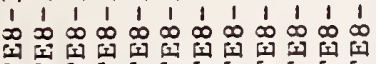

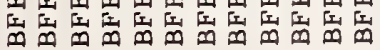

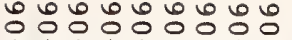
南南南南南南南南南

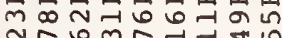

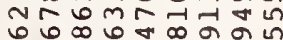

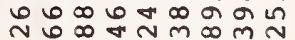

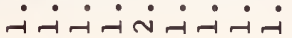

$N \infty m \infty N \infty \sim n$

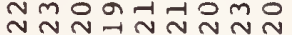

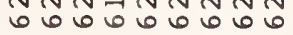
ல் 0000000

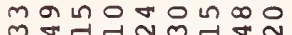

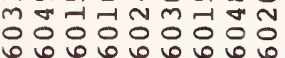
○ंல் $0 \dot{0} 0 \dot{0}$

อ N

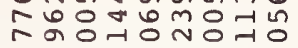
robarin

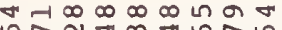
กิ

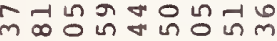
$\ddot{-i} \dot{\sim} \dot{\sim} \dot{\sim} \dot{\sim}-\dot{1}$

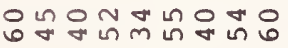

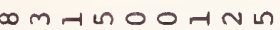
กัก๊ $\dot{\sim} \dot{0} \dot{\sim} \dot{\sim}$ N $\min \infty \mathrm{N} N$

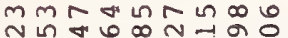

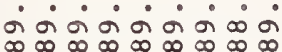
$\stackrel{\infty}{\sim} \stackrel{\infty}{\sim} \stackrel{\infty}{\sim} \stackrel{\infty}{\sim} \stackrel{\infty}{\sim} \stackrel{\infty}{\sim} \stackrel{\infty}{\sim} \stackrel{\infty}{\sim} \stackrel{\infty}{\sim}$

bon -ن் $\dot{\sim} \dot{0} \dot{0} \dot{-}$

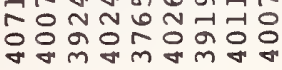
ㅇำ

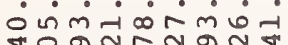

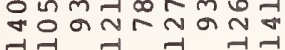

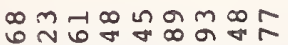
iं $\dot{\sim} \dot{\sim} \dot{-i} \dot{\sim} \dot{\sim}$

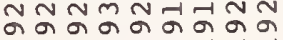

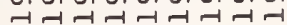

HNm $\operatorname{con}$ an

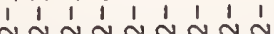
$\infty \infty \infty \infty \infty \infty \infty \infty$

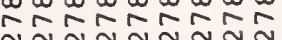

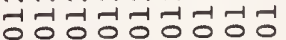
$\begin{array}{llllllll}1 & 1 & 1 & 1 & 1 & 1 & 1 & 1 \\ 1 & 1 & 1\end{array}$

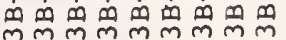
$\begin{array}{lllllllll}1 & 1 & 1 & 1 & 1 & 1 & 1 & 1 & 1 \\ 0 & 0 & 0 & 0 & 0 & 0 & 0 & 0 & 0\end{array}$

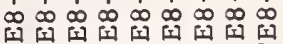

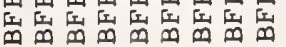

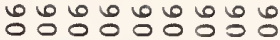
$+t+t+t+t+$

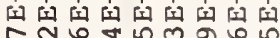

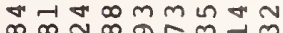
œ i-i-i $\dot{-i} \dot{-i}$

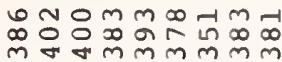

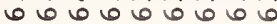
000000000

$m \infty \omega 0900 \infty$ NmMn

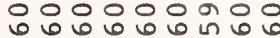
ல்

ron-ouata טำ (

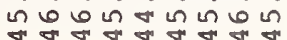

0 0 月

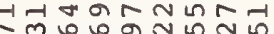

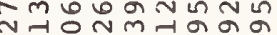
$\dot{\sim} \dot{\sim} \dot{\sim} \dot{\sim} \dot{-1}$

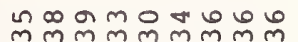

Na며유 งึส लंखिं $\dot{\sim}$ Nं

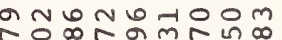
$\alpha \infty \infty \infty \infty$ a ब ก พ $\stackrel{\sim}{\sim} \stackrel{\sim}{\sim} \stackrel{\sim}{\mathrm{N}}$

nom

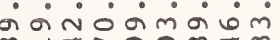
$\infty$ न

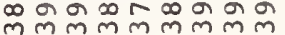

অำ

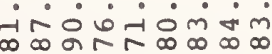

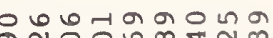
ลก $\dot{0} \dot{0} \dot{0} \dot{\circ} \dot{m}$

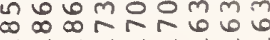

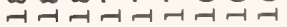

ง $\infty \infty \infty \infty \infty \infty \infty \infty \cdots$ बन नूनक ने

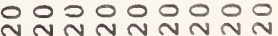
กัก กัก กัก กูก $\begin{array}{llllllll}1 & 1 & 1 & 1 & 1 & 1 & 1 & 1 \\ 1 & 1 & 1 & 1\end{array}$

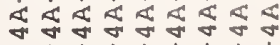
15. 150

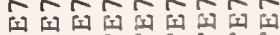

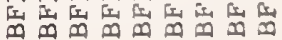

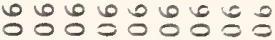

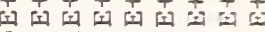
ㅇำ

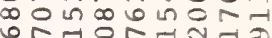

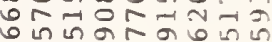
$-1-1=4$ (1)

둥ำ

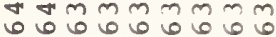
000000000

-man

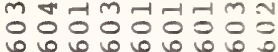
0000000 00

a d a in กูบ

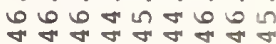

n जmR जm $\infty$ on न I N $\dot{-1} \dot{\sim} \dot{\sim} \dot{\sim}$

m

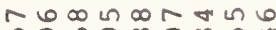
ด $\dot{\sim} \dot{N}-\dot{N} \dot{N}$

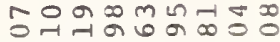
नं०ळar a o

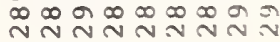

4h, - NoO नून न

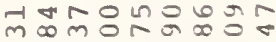
न

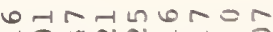
$\dot{0} \dot{0} \dot{0} \dot{0} \dot{0}$

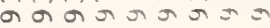

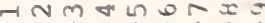
N N N N N N N

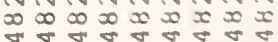
न N N N N N N N N 000000000 $\infty \approx \approx \infty \approx \pm= \pm$

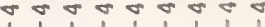
r T R T R T

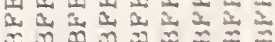


융유융요

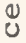
$4+4$ $\begin{array}{lll}4 & 4 \\ -1 & 0 & 0 \\ 0 & 0\end{array}$ $+t+t+t+t+t+t+t+t+t$

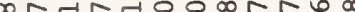
$m \sim ⿻ N$ क N

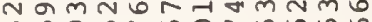
เก เก เก -i $\dot{-1} \dot{-1} \dot{-1} \dot{-i} \dot{-1}$

ONmmm

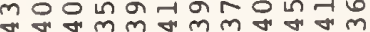

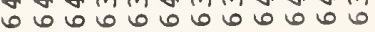
-

maन에

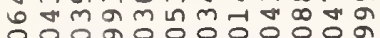

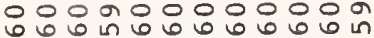

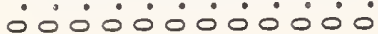

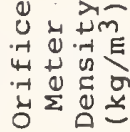

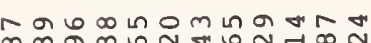
m . ก

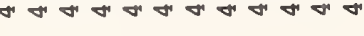
$\underset{0}{0}$ 先

ชั

is 300

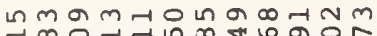

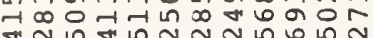
$\forall \mathfrak{*}$ เกี 质䓃嵒 4.?

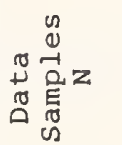

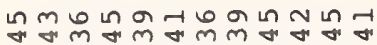

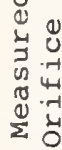

in - $\dot{i}^{m}$ 4 in 0

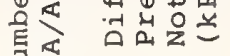

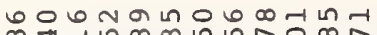
约 نें $\dot{\sim} \dot{m} \dot{\sim} \dot{\sim} \dot{\sim} \dot{m}$

$\omega$

(1) 过

EI

$\therefore 0$

范

है

อ

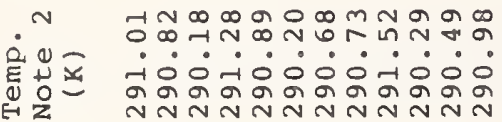

is -

a In andonthe (1) ㄴ.

$\dot{0} \dot{0} \dot{0} \dot{0} \dot{0} \dot{0} \dot{0}$ $\sim m \sim N \forall \infty \sim-1 \infty m \infty m$

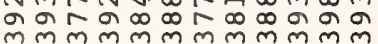

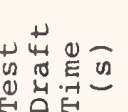

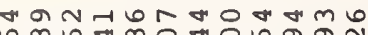

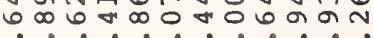
ம் $\dot{0} \dot{m} \dot{0} \dot{0} \dot{\sim} \dot{\sim} \dot{0} \dot{0}$

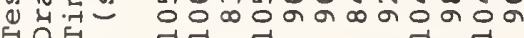

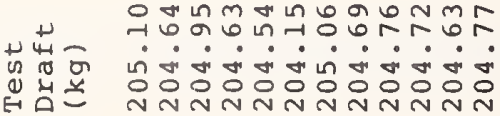

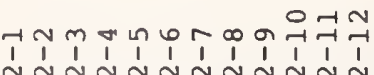
N N N N N N N N N

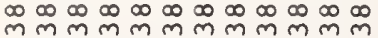

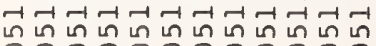
○0 0000000000

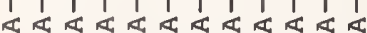

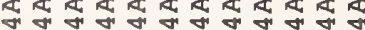

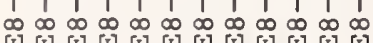

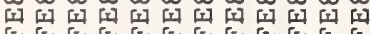

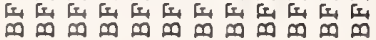

$\because 000600000$ $+t+t+t+t$

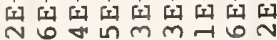

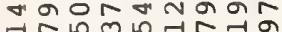

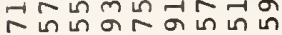

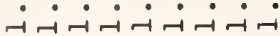

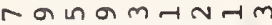
คิศ

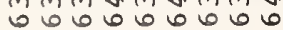
ல்ல0்0ல்

mNㄴ요용 ตกต สำำ

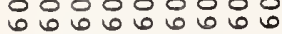
ட் $\dot{00} \dot{0} 0 \dot{0}$

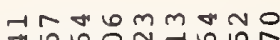

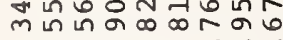
?? ? $\dot{0} \dot{0} \dot{m} \dot{m} \dot{\sim} \dot{\varphi}$

mก-1 L

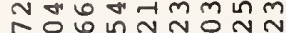
긍ํำสำำกำ vं

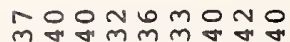

$m \infty n \circ-10+m$ mmm $\dot{i} \dot{\sim} \dot{\nabla} \dot{\sim} \dot{\sim} \dot{N}$

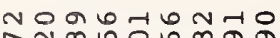
‥ $\infty$ a $\infty$ न $\stackrel{\infty}{\sim} \stackrel{\infty}{\sim} \stackrel{\infty}{\sim} \stackrel{\infty}{\sim} \stackrel{\infty}{\sim} \stackrel{\infty}{\sim} \stackrel{\infty}{\sim} \stackrel{\infty}{\sim} \stackrel{\infty}{\sim}$

0 Ln Un n vimir-idi

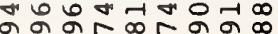
ले m लm m m m

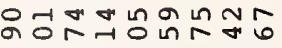
$\dot{\sim} \dot{m} \dot{m} \dot{\pi} \dot{m} \dot{N}$ ๗สু

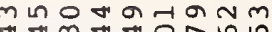
.

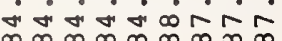

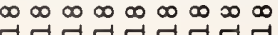

T)

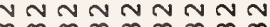
の

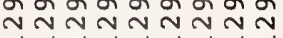

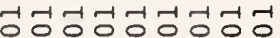
$1 \quad 1 \quad 1 \quad 1 \quad 1 \quad 1 \quad 11$

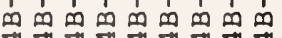

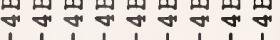
$\begin{array}{llllllllllll}1 & 1 & 1 & 1 & 1 & 1 & 1 & 1 & 1\end{array}$

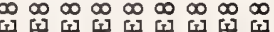

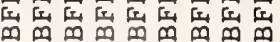


Table 6(a). Test Meter Identification and Physical Measurements Nominal 2 Inch AGA/API Orifice Meter (British Units).

$\begin{array}{ccccc}\text { Test } & \text { Test } & \text { Run } & \text { Orifice } & \text { Beta } \\ \text { Identification } & \text { Configuration } & \begin{array}{l}\text { Diameter } \\ \text { (inches) }\end{array} & \begin{array}{l}\text { Diameter } \\ \text { (inches) }\end{array} & \text { Ratio }\end{array}$

\begin{tabular}{|c|c|c|c|c|}
\hline $\begin{array}{l}\mathrm{BFEl}-4 \mathrm{~A}-072981-[9] \\
\mathrm{BFEl}-4 \mathrm{~A}-050582-[9] \\
\mathrm{BFEl}-4 \mathrm{~B}-050682-[8] \\
\mathrm{BFE} 2-4 \mathrm{~A}-011282-[5] \\
\mathrm{BFE} 2-4 \mathrm{~A}-011482-[1]\end{array}$ & $\begin{array}{l}1981-C \\
1981-D \\
1981-D \\
1981-D \\
1981-D\end{array}$ & $\begin{array}{l}2.0677 \\
2.0677 \\
2.0677 \\
2.0708 \\
2.0708\end{array}$ & $\begin{array}{l}1.1242 \\
1.1242 \\
1.1246 \\
1.1242 \\
1.1242\end{array}$ & $\begin{array}{l}0.5437 \\
0.5437 \\
0.5439 \\
0.5429 \\
0.5429\end{array}$ \\
\hline $\begin{array}{l}\mathrm{BFE} 2-4 \mathrm{~A}-011582-[9] \\
\mathrm{BFE} 2-4 \mathrm{~B}-011482-[8] \\
\mathrm{BFE} 1-5 \mathrm{~A}-073081-[9] \\
\mathrm{BFEl}-5 \mathrm{~A}-050682-[9] \\
\mathrm{BFEl}-5 \mathrm{~B}-111881-[9]\end{array}$ & $\begin{array}{l}1981-D \\
1981-D \\
1981-C \\
1981-D \\
1981-D\end{array}$ & $\begin{array}{l}2.0708 \\
2.0708 \\
2.0677 \\
2.0677 \\
2.0677\end{array}$ & $\begin{array}{l}1.1242 \\
1.1246 \\
1.3747 \\
1.3747 \\
1.3746\end{array}$ & $\begin{array}{l}0.5429 \\
0.5431 \\
0.6648 \\
0.6648 \\
0.6648\end{array}$ \\
\hline $\begin{array}{l}\text { BFEl-5B-050582-[9] } \\
\text { BFE2-5A-011382-[9] } \\
\text { BFE2-5B-011382-[9] } \\
\text { BFE2-5B-011582-[9] } \\
\text { BFEl-6A-072981-[8] }\end{array}$ & $\begin{array}{l}1981-D \\
1981-D \\
1981-D \\
1981-D \\
1981-C\end{array}$ & $\begin{array}{l}2.0677 \\
2.0708 \\
2.0708 \\
2.0708 \\
2.0677\end{array}$ & $\begin{array}{l}1.3746 \\
1.3747 \\
1.3746 \\
1.3746 \\
1.5003\end{array}$ & $\begin{array}{l}0.6648 \\
0.6639 \\
0.6638 \\
0.6638 \\
0.7256\end{array}$ \\
\hline $\begin{array}{l}\mathrm{BFEl}-6 \mathrm{~A}-073181-[9] \\
\mathrm{BFEl}-6 \mathrm{~A}-111881-[7] \\
\mathrm{BFEl}-6 \mathrm{~B}-073081-[9] \\
\mathrm{BFEl}-6 \mathrm{~B}-073181-[9]\end{array}$ & $\begin{array}{l}1981-C \\
1981-D \\
1981-C \\
1981-C\end{array}$ & $\begin{array}{l}2.0677 \\
2.0677 \\
2.0677 \\
2.0677\end{array}$ & $\begin{array}{l}1.5003 \\
1.5003 \\
1.5002 \\
1.5002\end{array}$ & $\begin{array}{l}0.7256 \\
0.7256 \\
0.7255 \\
0.7255\end{array}$ \\
\hline
\end{tabular}

Note: Test Identification bracket gives number of tests run with this geometry. A total of 154 tests were run on the nominal 2 inch run size. 
$+++++++++$

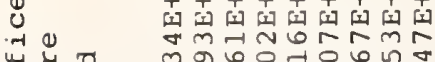

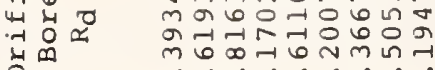
$\dot{-\dot{A}} \dot{\sim} \dot{\sim} \dot{\sim} \dot{-}$

vovinmbm 2

年

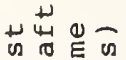
i.

in 2 का

1)

मा 20

这岕

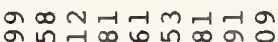
ด ก ๆ ن่

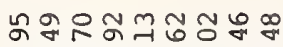
- تं

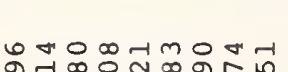
बन्त० 은ํำ

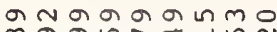

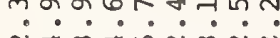

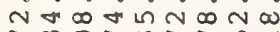
ขึำ

4
0
0

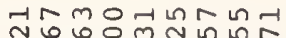

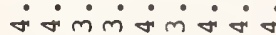

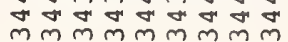

†

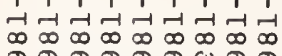
क न न के के NNNNNNNNN

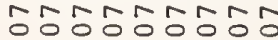
1 1 1111111

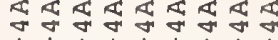
$\begin{array}{llllllll}1 & 1 & 1 & 1 & 1 & 1 & 1 & 1\end{array}$ 国国国国国国国

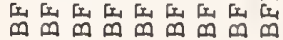

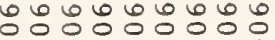
$++++++++t$

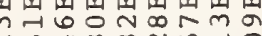
กำ ฟ

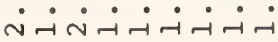

ก 000 In $\mathrm{mm}$ m N

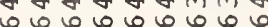
- 0000000

oammaーn I ช ซึનુ ○ंல்ட்0ல்

กลN N N

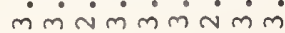

$m \omega m \infty m m N m$

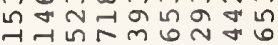

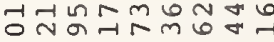
$\dot{\sim} \dot{-1} \dot{-} \dot{-} \dot{-} \dot{-} \dot{-}$

andmUnN

$m$ m

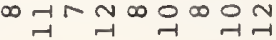

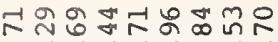
ஓं

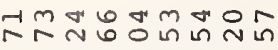
نं $ن \dot{\sim} \sim \dot{\sim} \sim \dot{0}$ ๑ 6 6 $606 \%$

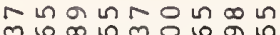
न-

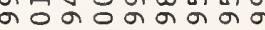
in 0 in 0 in in in in

GNARNALm ?.? -nNomimm and a d O m $\rightarrow N \rightarrow N$ NNNN

ค N N ने $\tilde{m} m \tilde{m} m \tilde{m} m m$

-1

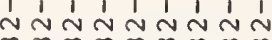
$\infty \infty \infty \infty \infty \infty \infty \infty \infty \infty$

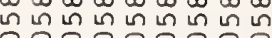
잉ㅇㅇㅇㅇㅇ

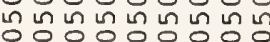
$\begin{array}{llllllllllllllllllll}1 & 1 & 1 & 1 & 1 & 1 & 1 & 1 & 1\end{array}$

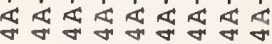
$\begin{array}{lllllllll}1 & 1 & 1 & 1 & 1 & 1 & 1 & 1\end{array}$

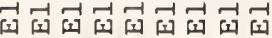

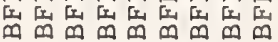
К HRnOmgma

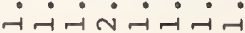

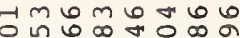

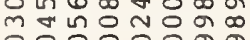
мंмंलिं $\dot{\sim} \sim$

பீ 南南南南南南南 món

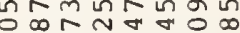
$m \infty m \sim \sigma \infty$ in - $-\dot{N-1}-\dot{1}$

$M N N \in \infty-10$

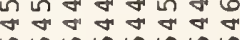
6660606

JMmONNN -1 66666666 ல0்0ல0்

mलबन

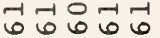
○ं0்

ㄸำ 근 न०कन क نं $\dot{\sim} \dot{N}$

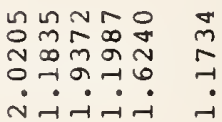

Tandoning

न न

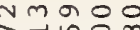

$m$

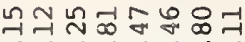

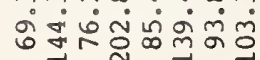

ㄲำ 즈ำ

n 우ㅇㅛㅛ

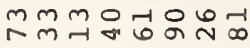
-iं $\sim \dot{\sim} \dot{-1}$ 06606606

$a n \infty N$ n oro $0 \mathrm{~N}$ -i் $\dot{0} \dot{0}$ กิ mo

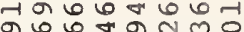
$\circ \dot{0} \dot{0} \dot{0}$ ㅁํํ요 웅은ํำ

लำ $\therefore \cdot \dot{0}$ 이요

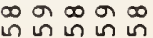

$\tilde{a}$
a
$\infty$
nn

HoRGGNm ㄱาง onormi-i MलNGOMOR

m웡ㅇㅇ $\infty \dot{\nabla} \dot{m} \dot{0}$ $000 m \infty$ mथm- mथNN

$-N \rightarrow N$ -

ํํ유ำ $\dot{0} \dot{0} \dot{0} \dot{0} \dot{0}$ onnG ले ले $\mathrm{m} \mathrm{m}$ ले $\mathrm{m}$ ले

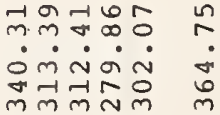

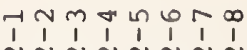
nN $N$ N $N$ N

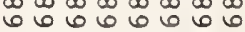
( 许 o o o o o o o $\begin{array}{llllllll}1 & 1 & 1 & 1 & 1 & 1 & 1 & 1\end{array}$

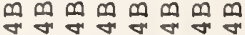
1 1 । 1111

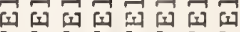

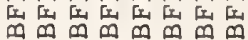

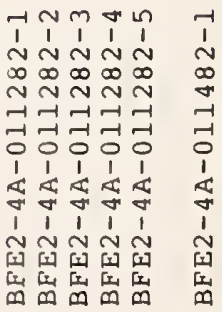




\section{-

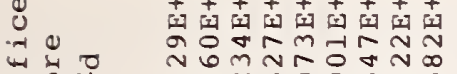

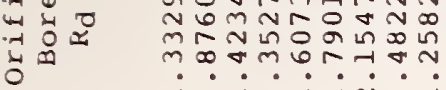

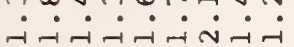

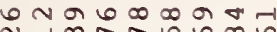
₹ 00000000

$m \infty m \sigma n \infty \infty i n$ ๑๐m $\dot{0} \dot{0} \dot{0} \dot{\sim} \dot{0} \dot{\sim}$

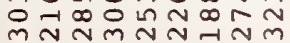

4 $\quad$ 유 ज乔

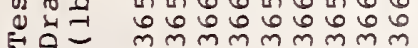

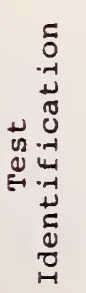

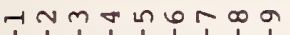
ง.ㄱำ

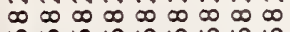
กก เก เก เก เก

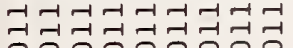
11 i 1

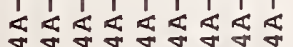
$\begin{array}{llllll}1 & 1 & 1 & 1 & 1 & 1\end{array}$

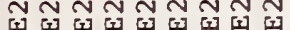
四红
ஜேะேะேะ $++t+++++$

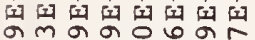
जल กิก mN-iंن-iनid

으유. -

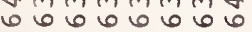
0000000

กㅎํㅇㅇㅇㅇㅛ

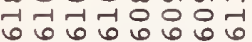
○ं 00000

는 ผี के नू नू नू ने ने พं山ं $\dot{\sim} \dot{\sim}$

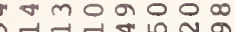
ब - 6 का $\mathrm{N} m+1$ નનન-નનન-1

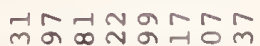
mo $\infty$ a ๓ 9

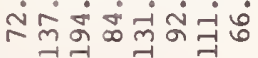

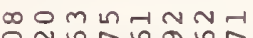
- $\dot{0} \dot{0} \dot{0} \dot{0}$ ชु

N $\ln 0 \ln \infty \mathrm{m} \rightarrow \mathrm{N}$ के ம0 जิ

N ก.? *

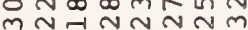

응요 ம் ตै

$\begin{array}{llllll}1 & 1 & 1 & 1 & 1 & 1 \\ 1 & 1 & 1\end{array}$ N N N N N N N

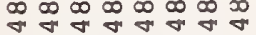
$\neg-1 \dashv-1-1$ 더엉ㄷㅇㅇㄷㅇㅇㅇㅇㅇ $\begin{array}{lllllll}1 & 1 & 1 & 1 & 1 & 1 & 1\end{array}$

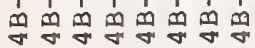

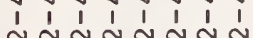

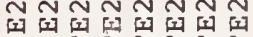

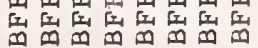

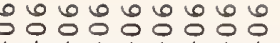
$t+t+t+t+t$

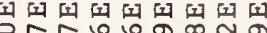
근

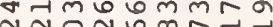

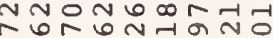
$\dot{\sim} \dot{\sim} \dot{-i} \dot{i} \dot{-i}$

N เก เก ต 证 赵

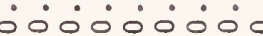

원

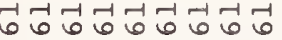
○ं0ல0் 00

०नr का m- क 용ㅎำ

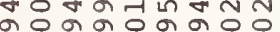
ㅍं $\dot{\sim} \dot{\sim} \dot{m}$

$m \infty$ o $\infty \pi \infty$ o 10

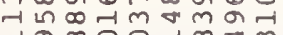

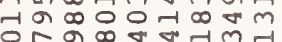
$\dot{m} \dot{\sim} \dot{\sim} \dot{\sim} \dot{\sim} \dot{-}$

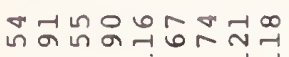

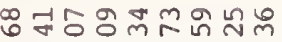
ه্-

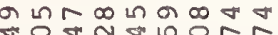
- $\cdot \dot{0} \cdot \dot{0} \dot{0}$ ชூ

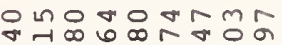
مिர்

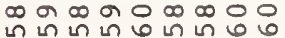

N 0,40 m o-in-íñ 근ำำกำ

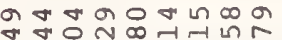
- $\dot{0} \dot{0} \dot{0}-\dot{\sim}$ 웅ㅇㅇㅇㅇㅇㅇㅛ 군 m m m m m m m m m m m

-1กm+

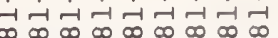
$\circ 00$ O 0 O 000 m m m m m m m m oñóñón $\begin{array}{llllllll}1 & 1 & 1 & 1 & 1 & 1 & 1 & 1\end{array}$

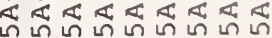
1 11111111

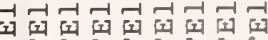

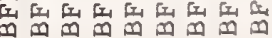

ஜே:

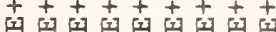

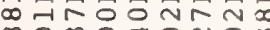

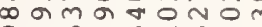

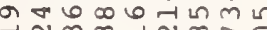

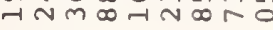
-กนกีก ก-่

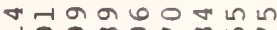
$\sigma \infty \infty \infty \infty \infty \infty \infty \infty$

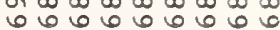
000000000

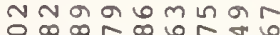
o $\infty \pi \infty 6 r+6$

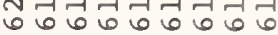
ல0ं0ல000

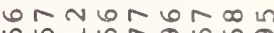
เึ乚

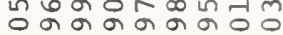
MN N N Nmm

o Nm $6 \mathrm{moln} \infty$ 约NNN

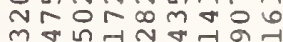
$\dot{\sim} \dot{\sim} \dot{m} \dot{\sim} \dot{m} \dot{-}$

กตัตก

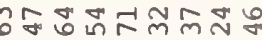

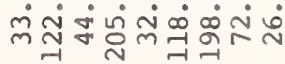

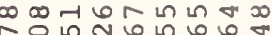
$\dot{0} \dot{0} \dot{0} \dot{0} \dot{0}-\dot{0}$

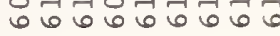

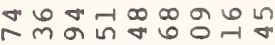
$\because \dot{\sim} \dot{\sim} \dot{0} \dot{0} \sim$

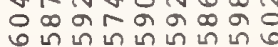

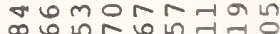
बंगेंत

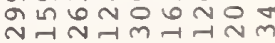

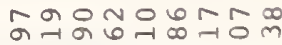

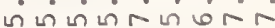

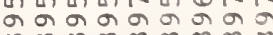

Anm

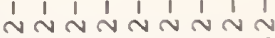

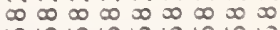

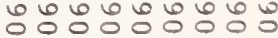

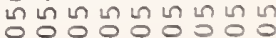

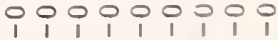

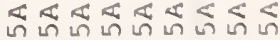
$\begin{array}{lllllllll}1 & 1 & 1 & 1 & 1 & 1 & 1 & 1 & 1\end{array}$

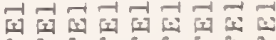

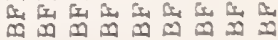


(1) ++++++++

$\rightarrow$ (1) N

फ प्र

प्रळ

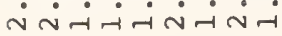

จำ 胫

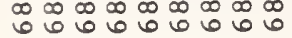
00000000

告

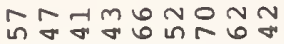
نे

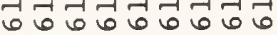
○ं0ல்0ல்

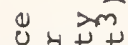
$\rightarrow$ a

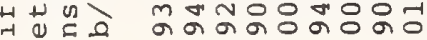
닝르

$\dot{\sim} \dot{\sim} \dot{\sim} \dot{m} \dot{m}$

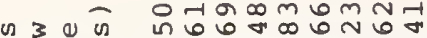

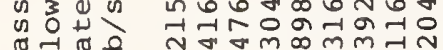

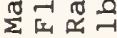

先点

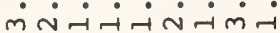

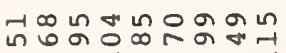

- mo

i. in

40 is

-1

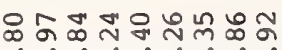

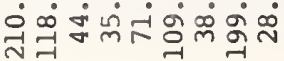

N

这岂的

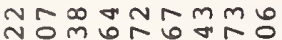

i) $z^{\circ}$

- $\dot{m} \dot{m} \dot{\sim} \dot{\sim} \dot{\sim}$

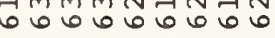

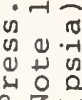

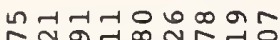

………ㄴ.?

○ $\ln -\infty m \pi$ n

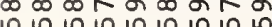

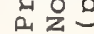

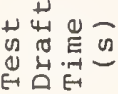

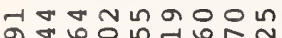
$\dot{0} \dot{0} \dot{0} \dot{\sim} \dot{0}$ नี่ ㄱำ

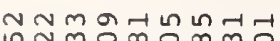

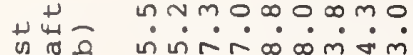

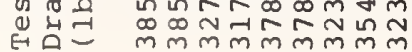

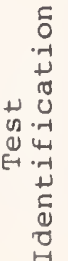

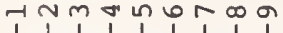

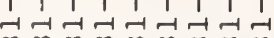
$\infty \infty \infty \infty \infty \infty \infty \cdots$ $\infty \infty \infty \infty \infty \infty \infty \infty \infty$ 거걱ㄱㄱㄱㄱㄱㄱㄱㄱㄱㄱ ન્નનન્નન્નનન્ન on on o o

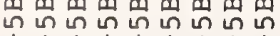
I

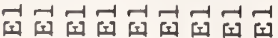

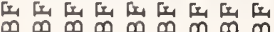

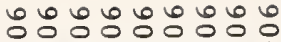
$+++t+t+t+$ vmm

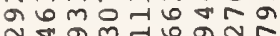
am $\mathrm{N} N-\mathrm{H}_{\infty} 0$ $\dot{\sim} \dot{\sim} \dot{-1} \dot{\sim} \dot{\sim}-$

ก $\infty_{\infty} \infty \infty \infty \infty \infty \infty \infty \infty \infty \infty$ 0006006006 000000000

이계

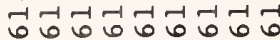
○ं0 0000.

N NmmnM-

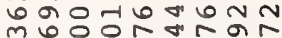

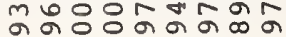
نंพmं $\sim \dot{\sim} \sim \dot{~}$

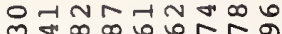
$N \cup m \infty \infty \infty 10$

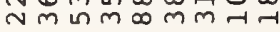
$\dot{\sim} \dot{\sim} \dot{-} \dot{\sim} \dot{\sim} \dot{m} \dot{~}$

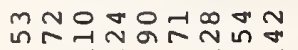

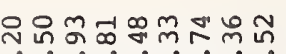

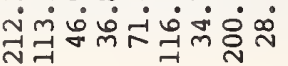

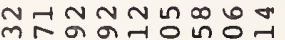
$\dot{-} \dot{\sim} \dot{\sim} \dot{\sim} \dot{\sim} \dot{v}$ ด ชิ

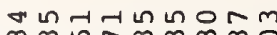
$\infty \infty n \pi m m \infty$ $-\infty 6$ bo -1 N क

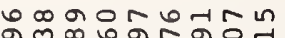
iri- $\dot{0} \dot{0} \dot{0}$ Nก⿵冂丶万ก

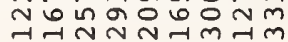

개의 MN $\dot{b} \dot{0} \dot{b} \dot{b} \dot{0} \dot{0} \dot{0}$ न न न न न न $\mathrm{mm} \mathrm{mm} \mathrm{mm}$

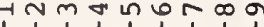
$\begin{array}{lllllllll}1 & 1 & 1 & 1 & 1 & 1 & 1 & 1 & 1 \\ 1 & 1 & 1 & 0 & 0 & 0 & 0 & 0 & 0\end{array}$

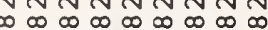
ก n 은은은으는은은 000000000 mo no on ตึ้ง $1,1,1,1$

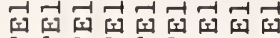

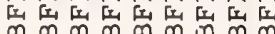

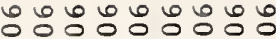

オี 수

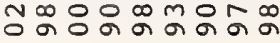
$\dot{m} \dot{\sim} \dot{\sim} \dot{\sim} \dot{\sim}$

๓ ผ v -i் $\dot{\sim} \dot{\sim} \dot{\sim} \dot{m} \dot{-}$

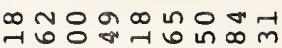

ஸึ

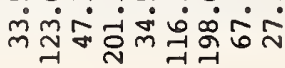

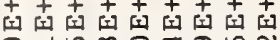

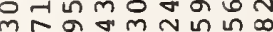
Nำ-i山्ن $\dot{\sim} \dot{\sim} \dot{\sim} \dot{-}$

$\infty \infty \infty \infty m \sim \infty m$ $\infty \sim \infty \infty \infty \sim \infty \infty$ . $60.0 \%$

のึm 궁ำ ல0் $000 \dot{0}$

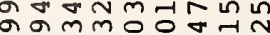
ㅇำ 6 ○ंல் $0 \dot{0} 0 \dot{0}$

ロ

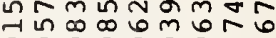

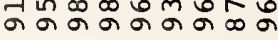
ำ $\dot{\sim} \dot{\sim} \dot{\sim}$

กกู๊

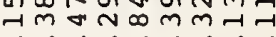
$\dot{~} \dot{\sim} \dot{-1} \dot{\boldsymbol{N}} \dot{\boldsymbol{m}} \dot{ }$

ด ก $* 6 \underset{-1}{0} \infty \omega_{-1} * m$

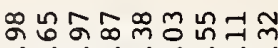

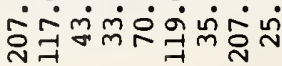

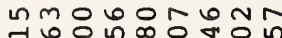
$\dot{\sim} \dot{\sim} \dot{\sim} \dot{\sim} \dot{\sim} \dot{\sim}$

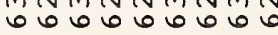

ㄲํㅇㅇํำ $\dot{\sim} \dot{\dot{r}} \dot{\dot{r}} \dot{\sim} \dot{\sim} \dot{\sim}$

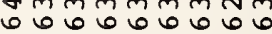

ดㄴำ $m m$ n $N \infty$ ก.?

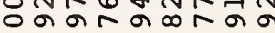
ชิ 幺ี่

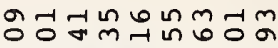
$\therefore \circ \dot{0} \dot{0} \dot{0}$ め in in in in in in in in in

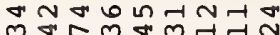
$\dot{0} \dot{m} \dot{0} \dot{0} \dot{0} \dot{0}$ 6 ก N

П $-\dot{-1} \dot{m} \dot{\sim} \dot{\sim}$ ㅁำ

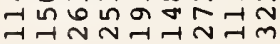

คำ 每

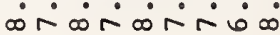

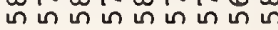
mmm mm mm

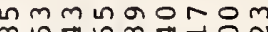

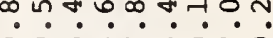
a $\sigma^{\circ}$ is m

भกm

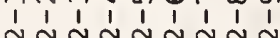
$\infty \infty \infty \infty \infty \infty \infty \infty \infty \infty$ mmmmmmmm ન્નુન્નુન

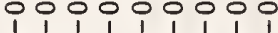

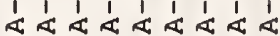

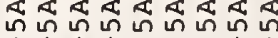

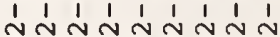

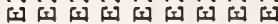

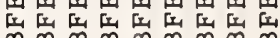

-1 $\mathrm{Nm}$ แn

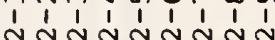
$\infty \underset{\infty}{\infty} \infty \underset{\infty}{\infty} \infty \underset{\infty}{\infty} \infty$ $\mathrm{mm} m \mathrm{~mm} m \mathrm{~m}$

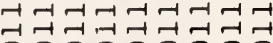
000000000

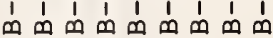

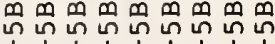

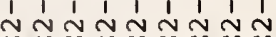

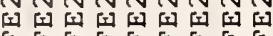

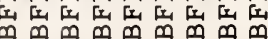




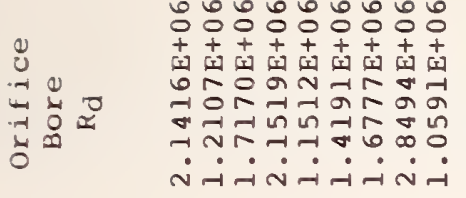

- angagnan

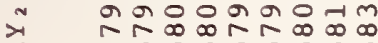

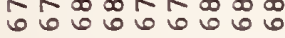
○ं் $\dot{0} \dot{0} \dot{0}$

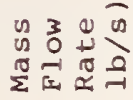

일

mo - is 0

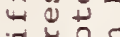

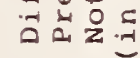

这

Aก ก ט.?

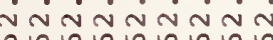

สิ กิ๊

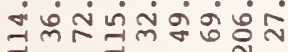

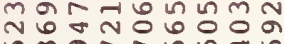
mañ $N-1+1+1$ ดु

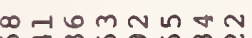

INGN

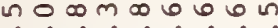

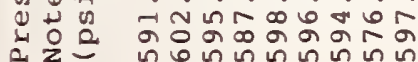

ע.

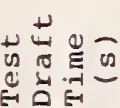

응요늄용

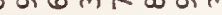

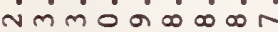

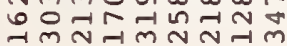

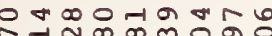
ก.บ 던 N6 n m잉ㅇㅇㅇㅇㅇㅇㅇㅇㅇ

HNm

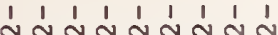

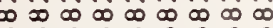
กี ถn

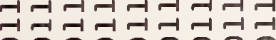

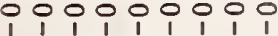
1 11111111 ตี in 1

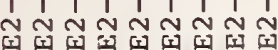

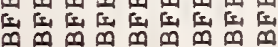
质质瓜瓜必

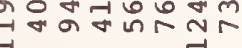

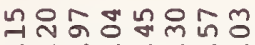

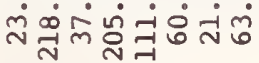

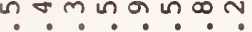
$\dot{0} \dot{0} \dot{0} \dot{0} \dot{0} \dot{0}$

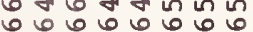

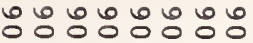

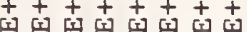
N $\infty N \cdots$ ฟ $\rightarrow m \sigma N F$

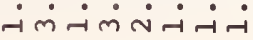

ด

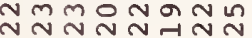
hnthrori 00000000

mกtam 다 다 ல $0 \dot{0} 0000$

줐이음

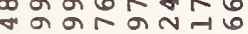
$0 \infty$ o $\infty$ \% 0 \% mivivini

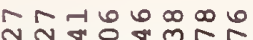
on mल mon a - $\forall$ m N

15

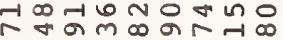
बma maरत्त

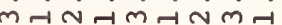

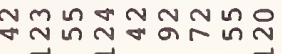

ํํㅇํำกำ คํํำ่

씨이업ㅇ욯ㅇㅇ

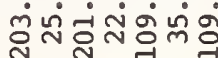

Haron แกก نंm $\dot{\sim} \dot{\sim} \dot{\sim} \dot{\sim} \dot{m}$ ०

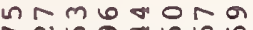
ก ก $\dot{0} \dot{0} \dot{m} \dot{\sim} \dot{\sim}$

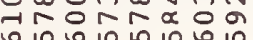

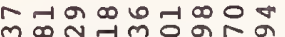
$\dot{0} \dot{0} \dot{0} \dot{0} \dot{0}$ ก

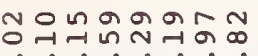
बंनं००००

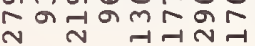

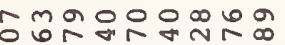
- $\dot{0} \dot{0} \dot{0} \dot{0} \dot{0}$ å N

그은 근 - óááo

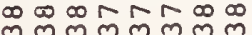

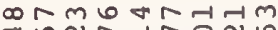
? ? ? ? ن 6 in $\ln$ in in $\infty \infty \infty \infty \infty \infty \infty \infty m$

-กตั

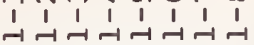

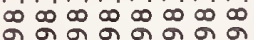
बून बून बे NNNNNNNNN Nóñóñ 1 1 1 1 1 1 1

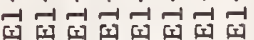

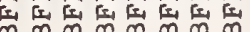

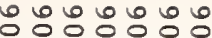
되 회 회 + t ++ - 0 (n) 60 in ปึ $\dot{m} \dot{m} \dot{-i} \dot{\sim} \dot{N}$

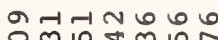
ํํำ $\stackrel{\sim}{\sim} \stackrel{N}{\sim}$ rrintrin 0000000

O $\infty \pi N$ a -1 $\therefore \circ \circ \circ \circ \circ$

- 6 n 2 m คิ $\infty \curvearrowleft$ พ $\dot{\sim} \dot{\sim} \dot{\sim}$

N $n$ nᄋำ भ๊̊ ด้ $m \rightarrow \dot{N} \sim \dot{\sim}$

สฺ ન

กก๊ anก. $\dot{m} \dot{\sigma} \dot{\dot{\sigma}} \dot{\dot{\sigma}} \dot{\sigma}$ ด 6 0 606

ㅁำกำ - ن $\dot{1} \dot{0} 0$ तa in in in in in in

$\infty$ a $\infty$ bo NRT $\ln$ in -1 oorinto ज न

$\operatorname{ran} 6$ in $\infty$ の ம $m$ m

HNm n

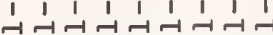

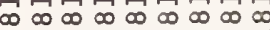

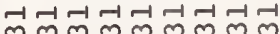
rarkraror 1

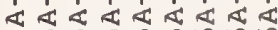

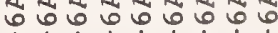
11111

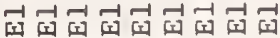

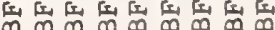

11

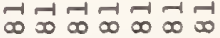
$\infty \infty \infty \infty \infty \infty \infty$

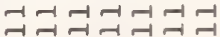

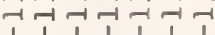

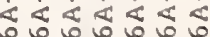
$1,1,1$

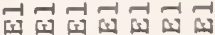

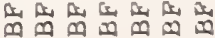




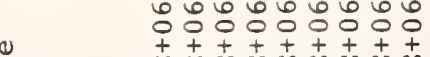

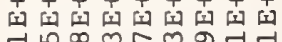 데 $\infty \cdots \cdots$ तr a 0 a $m-1$

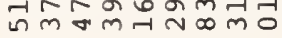 \\ نं $\dot{\sim} \dot{-1} \dot{m} \dot{\sim} \dot{m} \dot{-}$}

a N $20 N-1$ เ nNhNNNTNn O0000000

0

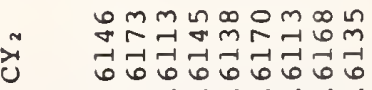
¿0ं0்

ن. $\rightarrow-1,0+4$

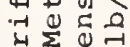

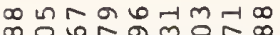

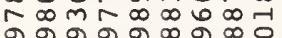
न न न न न $\infty$ ने $\infty$ ० نं $\dot{\sim} \dot{\sim} \dot{\sim} \dot{\sim}$

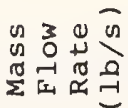

$\infty$ a กับ

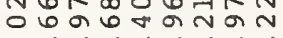

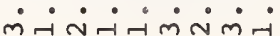

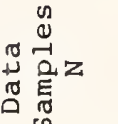

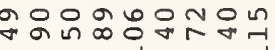
ก

- $m \overline{0}$

4 in

400

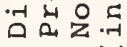

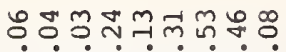
元

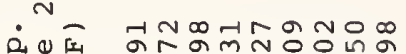
实证。

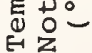

$\dot{\dot{\sigma}} \dot{\sim} \dot{\sigma} \dot{\sim} \dot{\sim} \dot{\sim} \dot{0}$

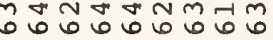
in 0

$\infty \pi \infty-1 a \infty-1 \operatorname{nn}$ $\forall \infty \infty \circ \cdots n$ ก

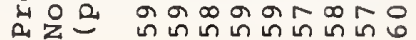

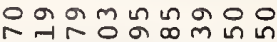

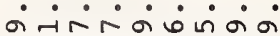
बूत

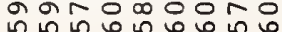

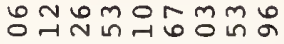

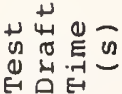
点

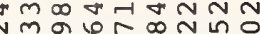

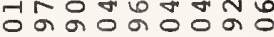

Nลี

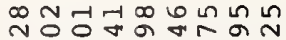
im

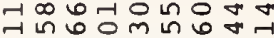

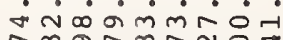

ช้ำำำำ

H

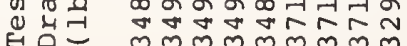

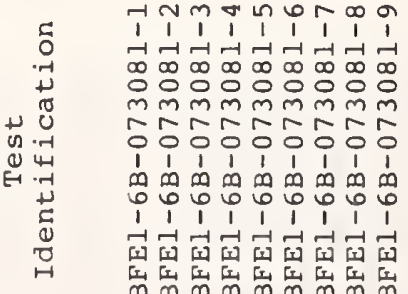

HN⿴囗十

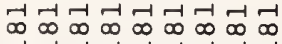

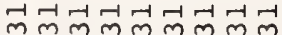
ñNañón

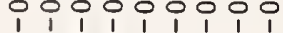

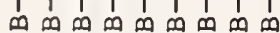

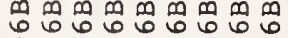

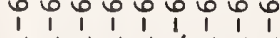

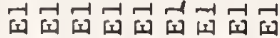

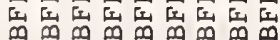

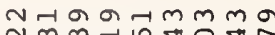
* : ? : ?

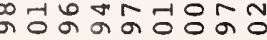
牙是 
Table 7(a). Test Meter Identification and Physical Measurements Nominal 3 Inch AGA/API Orifice Meter (British Units).

Test

Identification

$\mathrm{BFE} 3-2 \mathrm{~A}-011982-[8]$

$\mathrm{BFE} 3-2 \mathrm{~B}-012182-[9]$

$\mathrm{BFE} 4-2 \mathrm{~A}-070782-[6]$

$\mathrm{BFE} 4-2 \mathrm{~B}-070782-[4]$

$\mathrm{BFE} 4-2 \mathrm{~B}-070982$ - [ 9 ]

BFE3-3B-012282- [9]

$\mathrm{BFE4}-3 \mathrm{~A}-061981-$ [ 9 ]

BFE 4-3A-072381-[9]

$\mathrm{BFE} 4-3 \mathrm{~B}-061881-[9]$

BFE4-3B-072281-[9]

$\mathrm{BFE} 3-4 \mathrm{~B}-011982-[9]$

$\mathrm{BFE} 3-4 \mathrm{~B}-012282-[9]$

$B F E 4-4 A-061981-[9]$

$\mathrm{BFE} 4-4 \mathrm{~A}-072181-[9]$

BFE4-4B-070282-[9]

BFE 3-5A-0.22082- [8]

BFE3-5B-012082-[9]

$\mathrm{BFE} 4-5 \mathrm{~A}-070282-[9]$

$\mathrm{BFE} 4-5 \mathrm{~B}-070882-[10]$

$\mathrm{BFE} 3-6 \mathrm{~B}-012182-[8]$

BFE 4-6A-061781- [9]

$B F E 4-6 A-072281-[9]$

BFE4-6B-070882-[9]
Test

Configuration
Run

Diameter

(inches)

3.0679
3.0679
3.0678
3.0678
3.0678

3.0679

3.0678

3.0678

3.0678

3.0678

3. 0679

3. 0679

3.0678

3.0678

3. 0678

3.0679

3.0679

3.0678

3.0678

3.0679

3.0678

3. 0678

3. 0678
Orifice

Diameter

(inches)

1. 1250

1.1249

1.1250

1.1249

1.1249

1.4997

1.5000

1.5000

1.4997

1.4997

1.7498

1.7498

1.7503

1.7503

1. 7498

2.0006

1.9998

2.0006

1.9998

2.2496

2.2492

2. 2492

2.2496
Beta

Ratio

0.3667

0.3667

0.3667

0.3667

0.3667

0.4888

0.4890

0.4890

0.4889

0.4889

0.5704

0.5704

0.5705

0.5705

0.5704

0.6521

0.6519

0.6521

0.6519

0.7333

$1981-\mathrm{B}$

1982-A
0.7332

0.7332

0.7333

Note: Test Identification bracket gives number of tests run with this geometry. A total of 197 tests were run on the nominal 3 inch run size. 
0

$+$

岂

है

व

$+.-1$

ป

元

ن

元

ग

(1)

马ु 4

(1)

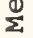

$-2$

है

己

崩

E)

$\therefore$

용

ㄷ.

(1) 0 웅 है
ஜ유:\%゚

$2+t+t+t+t$

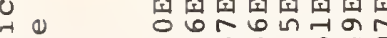
世 मू 0 里

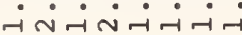

$\infty \infty \omega-1 m$ a $\infty$

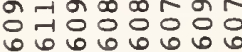
- $\dot{0} \dot{0} \dot{0} \dot{0} 0$

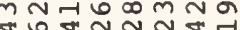

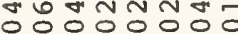
$6606060 \%$

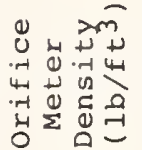
00000000

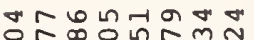
$\infty$ in बन बूनून बून

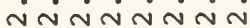

⿰纟及卩 ชูర

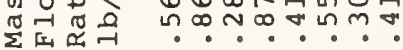
党 -1ન-1ન-ંન-

$H m$ แn $n=\infty \infty$ न1

- $m \widehat{O}$ + in 2 告 4 a

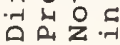

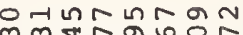
$\dot{0} \dot{i} \dot{0} \dot{0}$ mNnmmi

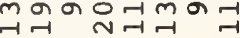

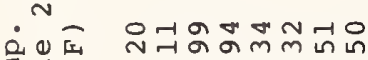

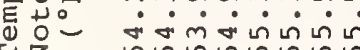

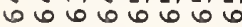

in $\pi$

(1) य $\dot{a} \dot{i} \dot{\sim} \dot{\sigma}$

แ

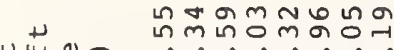

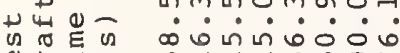

(1)

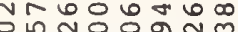

फ

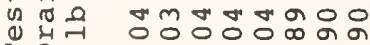

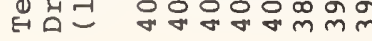

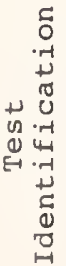

HNmanor ง.ง $\infty \infty \infty \infty \infty \infty \infty \infty$ क न न बून क

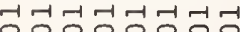
1 1 1 1 1 1 1 1

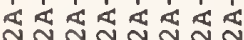

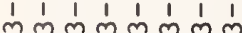
$m m m m m m$

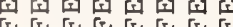

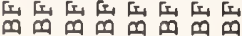

○ $+t+t+t+t+$

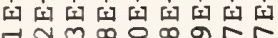
게요

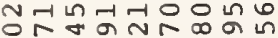
ज $0 \wedge m-1$ in $m$ -i-i-i $\dot{-i} \dot{\sim} \dot{-}$

and

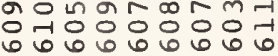
ல0ல0ல0ல0்

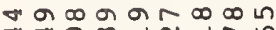
ฟ

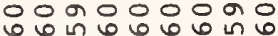
000000000

:ะ:\%: $+t+t+t$ ब 007 व ก $\operatorname{N} \infty \infty$ on $\infty$ in $\infty \circ \sim O N$ - $\dot{\sim} \dot{\sim} \dot{-1}$

NNon

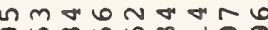

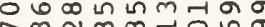
10 00 a 00 \% न $\dot{m} \dot{m} \dot{m} \dot{\sim} \dot{m} \dot{N}$ $\infty$ ब

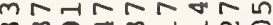
$m m$ m $m$ 대

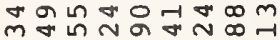
i- $\dot{-i} \dot{-i} \dot{-} \dot{-} \dot{-}$

$\infty 0-10 m-1 \sim m$ \%

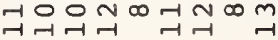

กั

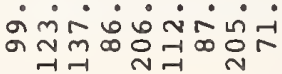

$\infty$ Nanm $\infty$ n $n$

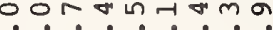
نि $\dot{0} \dot{0} \dot{\circ} \dot{\circ} \dot{\sigma} \dot{a}$

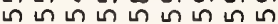

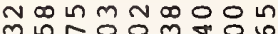
ن் $\dot{0} \dot{0} \dot{\sim} \dot{\sim} \dot{\sim}$

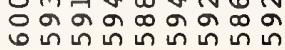

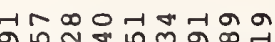
on $n$ ज +oumminti N N N N N

ด $\dot{\sigma} \dot{\infty} \dot{\infty} \dot{\infty} \dot{\sim} \dot{\sim}$

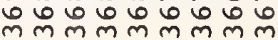

กับ $\infty \infty_{\infty}^{\infty} \infty \sim_{\infty}^{\infty} \infty \infty_{\infty}^{\infty} \infty$ N $\underset{\sim}{\sim} \sim \widetilde{N} \sim \widetilde{N}$ 겅더어어어더더덩

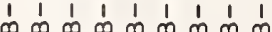

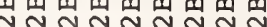

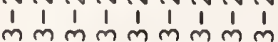

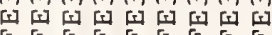
岳品品品品品品 $\infty$ NTR M $\begin{array}{llllll}0 & 7 & 0 & 0 & 0 & 0 \\ 0 & 0 & 0 & 0 & 0\end{array}$ -0்0ं0

กตก 느융ㅇ 웅ㅇำ 000000

n $\operatorname{nem} n$

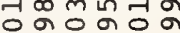
$\dot{m} \dot{m} \dot{\sim} \dot{\sim}$

웅ํํ뉴 76.000 $\therefore \circ \circ 0^{\circ}$

뇨용 궁용 ㅇํㅇำ ○ं०்

$\infty$ or n. $\dot{m} \dot{m} \dot{m}$

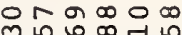
กิ సैळ

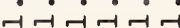

$\neg 0 \infty 0$ 능욘ㄷำ 앙ํำ -i $\dot{-1} \dot{-1}$

음ํㅓㅁㅇㅛ

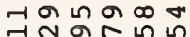

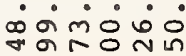
न $\because \infty \nabla \square m$ वंळ

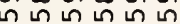

ํํㄴ ம் $\dot{0} \dot{\sim} \dot{0}$

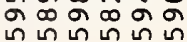

$m m N n \infty$ $r \infty \infty$ om $\infty \dot{0} \dot{0} \dot{0}$ ข้ กับ

N H m $\mathrm{m} \tilde{m} \bar{m}$

$-1 N m \nabla n$ ป $\infty \infty \infty \infty \infty \infty$ r. ntr inorion 000000

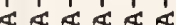
N $\sim \widetilde{N} \sim \widetilde{\sim}$

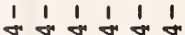
ज्ञ 되 되 되 되 品品品至品品

कून๊ำ

-1 $\ln _{0}^{\infty}$

$\therefore \dot{0} \dot{0}$

aำ $\ln _{-1}^{\infty}$

$0 \infty \infty$ in ข $\infty m$ 0ंक

응의

$0 \infty 09$

r.t 얻ำ NN

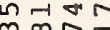
m

$\pi n m a$ n. $\infty \infty \infty \infty$ กิำ 응ㅇㅇㅇㅛ

어이 向品 N N का क ज्ञ

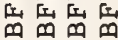

$\infty 0 \pi n$
:ะะะ +1 $\infty$ क $\infty$ $\infty 10$ $\forall$ in

$\dot{-1} \dot{-1}$

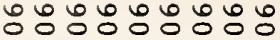

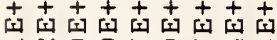
त ल n $\infty N \infty$ N $\approx$ \% $N$ N $\dot{-\dot{A}} \dot{-} \dot{\sim} \dot{\sim} \dot{\sim} \dot{\sim}$

Non onthrnghr

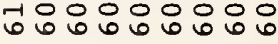
வ0் $00000 \dot{0}$

$\sim m \sim ⿻ 4-1) \infty$

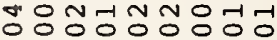

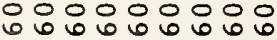
000000000

NN"กด น

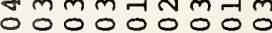
$\dot{m} \dot{m} \dot{m} \dot{m} \dot{m} \dot{m} \dot{m}$

MNกNON N mํำ -ં $\dot{-1} \dot{-1} \dot{-} \dot{-} \dot{-} \dot{-1}$

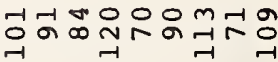

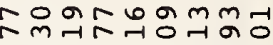
नंनंबंक

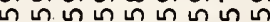
$\therefore \dot{0} \dot{\sigma} \dot{\sigma} \dot{\infty} \dot{0} \dot{\sigma} \dot{\infty} \dot{m}$

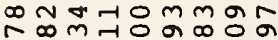

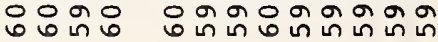

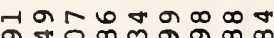
. iotan $N$ N ํㅝㅁำ

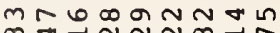

$\sim \infty \infty \infty \infty \infty \infty \infty$

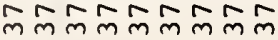

nmonoroma ป่ง $\infty \infty \infty \infty \infty \cdots$ 


\section{0 $\begin{array}{ll}-1 & 0 \\ - & 0 \\ 0 & 0\end{array}$} $\check{z}$

m몌욤ำ

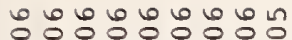
$\begin{aligned} & + \\ & +\end{aligned}++t+t+$ ब 6060000 ธน

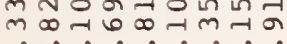
-

ก ก ก

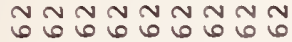
○ं $0 \dot{0} 0 \dot{0} 0$

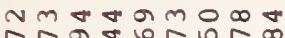
¿ี

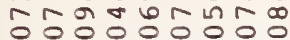
666666666 ○ं $0 \dot{0} 000$

\section{im} $\rightarrow 0.1=$ 4 ज 0

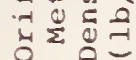

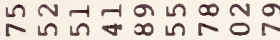

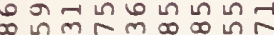

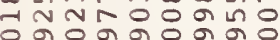
लंखिं $\dot{N} \dot{N} \dot{m}$

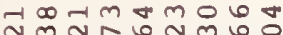
ำกNํำก ०m mom $m$ in $\dot{-1} \dot{\sim} \dot{\sim} \dot{m} \dot{-1} \dot{\sim}$

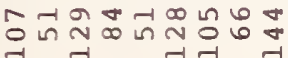

moOn man ก $\infty \infty \pi \curvearrowleft \curvearrowleft \infty \infty$ $\dot{m} \dot{0} \dot{0} \dot{0} \dot{\sim} \dot{\sim}$ * an

ผ ํำน சं

$6 \infty m \circ N+N \backsim \infty$

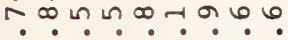
oramanna

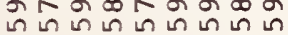
क人⿻上丨 금의

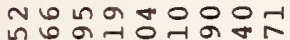

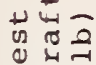
$\Leftrightarrow \stackrel{2}{0}=$

†1 ก

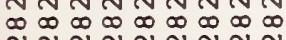
N N N N N N N

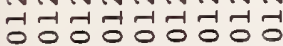
1 1 1 1 111 1 1

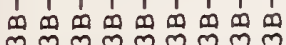
$\begin{array}{lllllllll}1 & 1 & 1 & 1 & 1 & 1 & 1 & 1 & 1\end{array}$ $m m m m m m m$

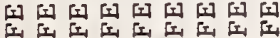

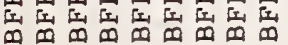

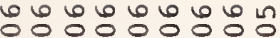
$+t+t+t+t+t$

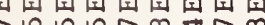
กㄱำ

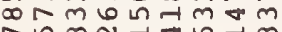

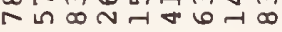

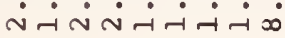

O6 an

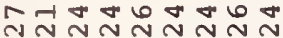

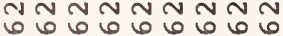

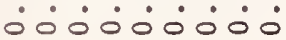

$\infty 6 \infty \in N O N N-1$ 品

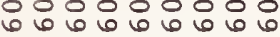
○ं $\dot{0} \dot{0} 0 \dot{0}$

이맘요 mनન

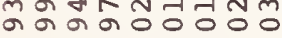
Nं $\sim \dot{m} \dot{m} \dot{m}$

$6 \infty \sim ⿻ 上-1)$

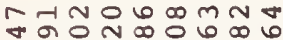

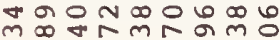
$\dot{m} \dot{-\dot{v}} \dot{\sim} \dot{-\dot{H}} \dot{-\dot{-}}$

ㅇำ $\infty$ ๙

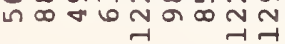

om $\ln$ ONHM G สำ

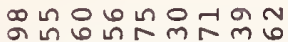
-ivi $\dot{\sim} \dot{\sim} \dot{\sim} \dot{\sim}$ สำ

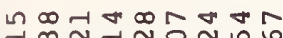
กmก!กㄴ.?

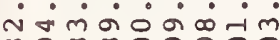

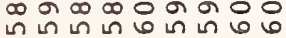

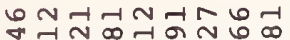
बं-

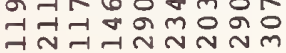

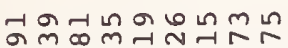

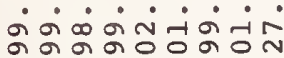

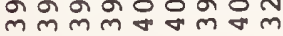

-1

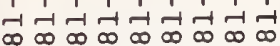
ఐ

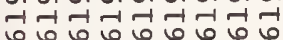
$\circ 0: 000000$ $\begin{array}{llllllllllll}1 & 1 & 1 & 1 & 1 & 1 & 1 & 1 & 1\end{array}$

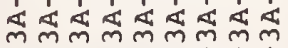
$\begin{array}{lllllllll}1 & 1 & 1 & 1 & 1 & 1 & 1 & 1 & 1 \\ 4 & + & 4 & 4 & 4 & 0\end{array}$ 国私四国国国国

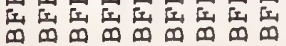

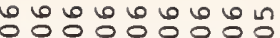
$+t+t+t+t$

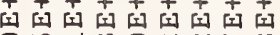

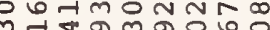
당 ง

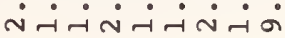

Nan

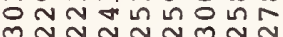

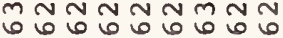
○ं0் 0000

ดก에 $\infty \infty \omega 6$

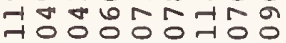
$60606066 \%$ 000000000

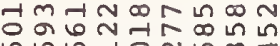

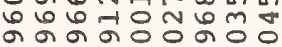

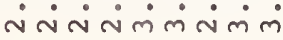

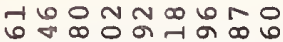

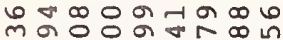
mลㅇํㅇำ $\dot{\sim} \dot{\sim} \dot{\sim} \dot{-} \dot{\sim} \dot{-}$

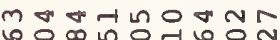

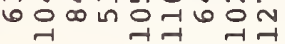

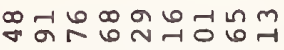
$\dot{0} \dot{0} \dot{0} \dot{0} \dot{\sim} \dot{\sim}$

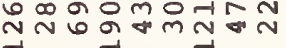

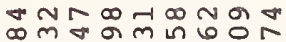
ง

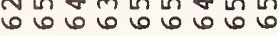

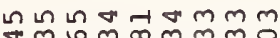
+ $m$ ?

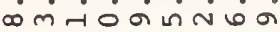
a a $\infty$ aㅇㅇㅇ

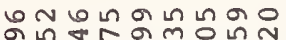

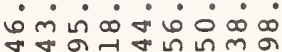

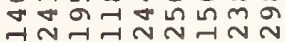

요요 $\infty \infty$ 에

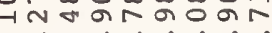
N $N$ N-IMN

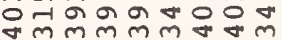

INm ㄴ $\begin{array}{lllllllll}1 & 1 & 1 & 1 & 1 & 1 & 1 & 1 & 1\end{array}$

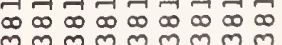
NNNNNNNNN nathastio OOO OOOOOO

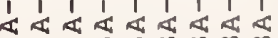
लेल लॉल ल ल

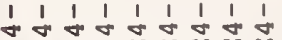

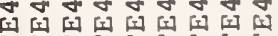

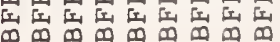

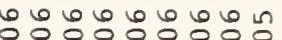

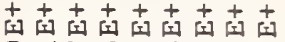
그음 政 * -

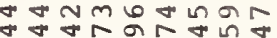

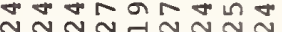

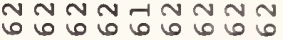
வ0000000

mm-1

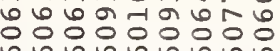
. . . . . . $\therefore 0000000$

ด ก 느읭ㅇ은

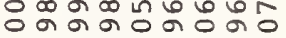

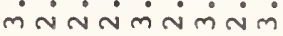

이의의문 6워

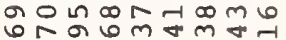
$\dot{-i} \dot{\sim} \dot{\sim} \dot{\sim} \dot{-1} \dot{-}$

응

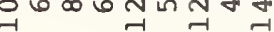

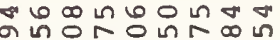
$\dot{-} \dot{-i} \dot{\sim} \dot{-} \dot{-} \dot{\sim}$

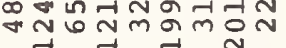

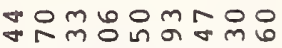
-10

ㄱㅇำ

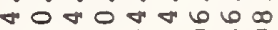
హ゙

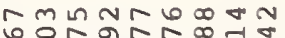
のंவ்

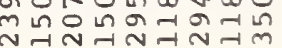

H이네

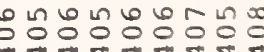

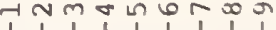
$\begin{array}{lllllllll}1 & 1 & 1 & 1 & 1 & 1 & 1 & 1\end{array}$ $\infty \infty_{\infty}^{\infty} \infty \infty^{\infty} \infty x^{\infty} \infty x^{\infty} \infty$ $\infty \infty \infty \infty \infty \infty \infty \infty \infty$

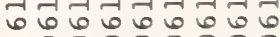
00000000 $\infty \infty$ $\begin{array}{llllllll}1 & 1 & 1 & 1 & 1 & 1 & 1 & 1\end{array}$ के

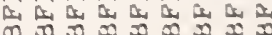


*

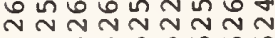
666660606 ○் $\dot{0} \dot{0} \dot{0} \dot{0} 0$

$m \pi n \pi 0$

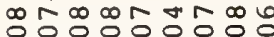

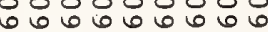

¿ं0் $000 \dot{0}$

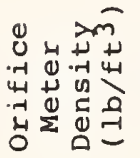

ம요ำ

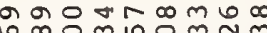

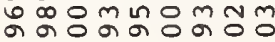

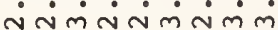

क 3 का के

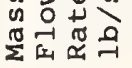

a $6 \pi \infty 606$ n N N잉 $\dot{\sim} \dot{-} \dot{\sim} \dot{\sim} \dot{-} \dot{m} \dot{-1}$

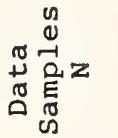

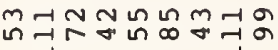
- $m \widehat{0}$ -4 is 0 $40+$

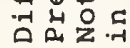
$N$ 递芒的

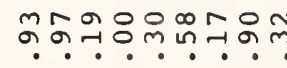
$\dot{i} \dot{\sim} \dot{\sim} \dot{-i} \dot{\sim} \dot{\sim}$

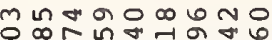
$\therefore \dot{0} \dot{0} \dot{0} \dot{0} \dot{0}$ กับ

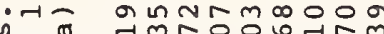

in 0.7

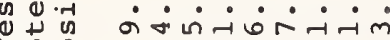

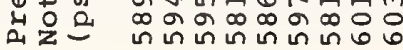
㟧䓵的

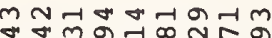
ำ $\dot{\sim} \dot{0} \dot{0} \dot{0} \dot{0} \dot{0}$

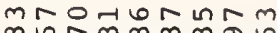
or $\dot{0} \dot{0} \dot{0} \dot{0}$ mm mm mm m $m$ m $m$ N

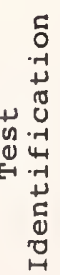

กตั $\begin{array}{ccccccccc}1 & 1 & 1 & 1 & 1 & 1 & 1 & 1 & 1 \\ -1 & -1 & -1 & -1 & -1 & -1 & -1 & -1 & 1\end{array}$ $\sim \infty ⿻ \infty \sim^{\infty} \infty \infty^{\infty} \infty \infty \infty$ กNกNกNNNN vinnnNNNN OOOOOOOOO m品品息息品

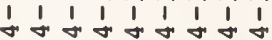

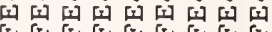

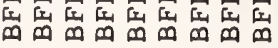

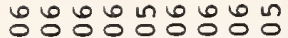
$++t+t+t+t$

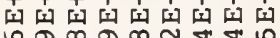
- 000

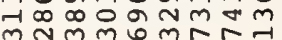
$\dot{m} \ddot{\sim} \dot{\sim} \dot{\circ} \dot{\oplus} \dot{-} \dot{-\infty}$

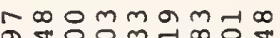

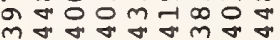

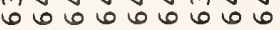
ல0000000

OnNUmOGm

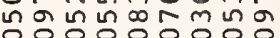

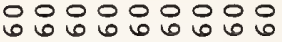
வ $\dot{0} \dot{0} \dot{0} \dot{0} \dot{0}$

o $\backsim m \not m m m \infty \forall$

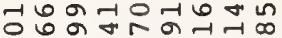

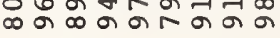
กักักักัก

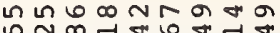
กำ

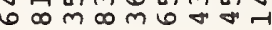
$\dot{-i \dot{m}} \dot{-i} \dot{\sim} \dot{\sim} \dot{\sim}$

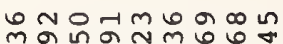

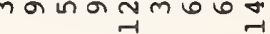

กที

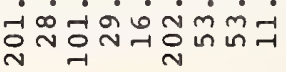

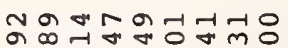
$\dot{m} \dot{\dot{\sigma}} \dot{\dot{\sigma}} \dot{0} \dot{0} \dot{0}$

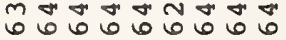

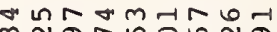
ก ம जै कर के एक के

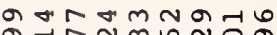

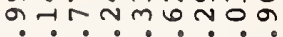

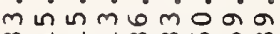

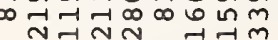

mm $m$ -

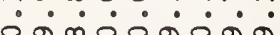
잉ㅇㅇㅇㅛ m m m mm m m

-1ำ ป $\infty \infty \infty \infty \infty \infty \infty \infty \infty$

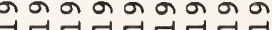
겅겅덩겅덩겅겅 $\begin{array}{llllllll}1 & 1 & 1 & 1 & 1 & 1 & 1 & 1\end{array}$ 四的的的向

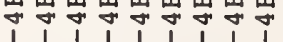
Mmmmmmm

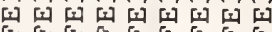

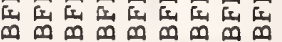

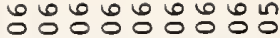
$++++++++$

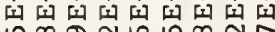
ก

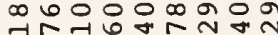
ma nonna $\dot{m} \dot{i} \dot{\sim} \dot{-1} \dot{-1} \dot{n}$

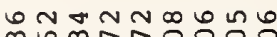

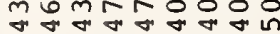
ช ซ ○் $\dot{0} 0 \dot{0} \dot{0} 0$

검ㅇㅇㅇㅛ $\infty=1 \infty N$ ก

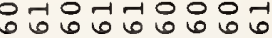
ல0ं0ல0ல00

nNRTNan-m

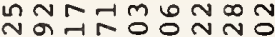

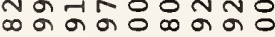

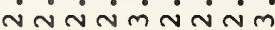

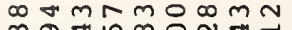
maテ幺m: Non $\dot{\forall} \dot{\sim} \dot{-} \dot{\sim} \dot{\sim} \dot{-}$

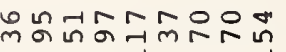

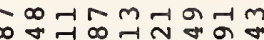
- $\cdot 0 \cdot \dot{0}$

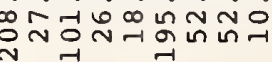

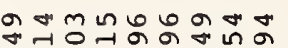

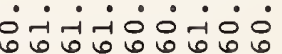

- $N$ Nm

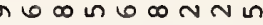
$\dot{i} \dot{0} \dot{0} \dot{0} \dot{0}$

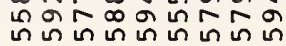

minotmin $-\infty N-1 \infty \infty m \backsim N$ $\dot{m} \dot{-} \dot{\sim} \dot{m} \dot{0} \dot{\sim} \dot{0}$

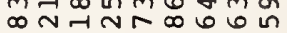
NָสNำ

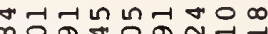
irirario

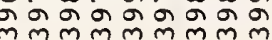

HNM

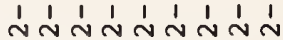

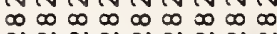
NNNNNNNN

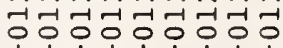
$1,111,111$ $m \infty n$

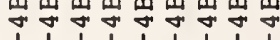
रे

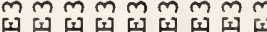

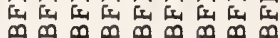

붕ํㅇ유요 $++t+t+t+t$

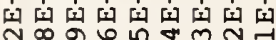
No অิ $\dot{-i} \dot{\sim} \dot{\sim} \dot{\sim} \dot{\sim} \dot{\sim} \dot{\infty}$

の

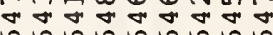

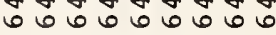
ல் $\dot{0} 0 \dot{0} 0 \dot{0}$

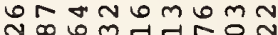

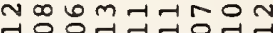
660666066 ○ं $\dot{0} \dot{0} \dot{0} 0 \dot{0}$

은

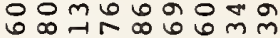

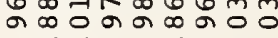
NNMNNNNMm

무뉴요

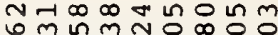
$\forall n \infty m+\infty N$ in

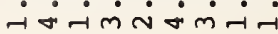

ํำ จำㄴำㄱำ

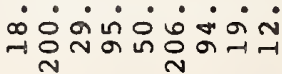

ตัન -i்-i் $\dot{-1} \dot{0} \dot{0}$ 6 in 6066060

am an GN ง $6 \circ \mathrm{m}$ in $\sim \dot{\sim} \dot{-i \infty} \dot{0} \dot{0}$

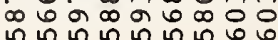

ฟลกดm का

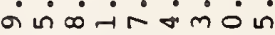
ก

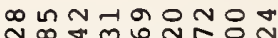
$\therefore \dot{0} \dot{0} \dot{0} \dot{0} \cdot$

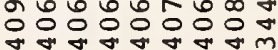

I

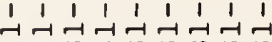

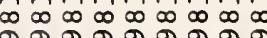

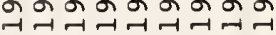
6ซื 6666666

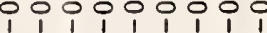

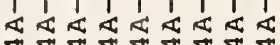

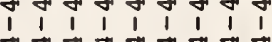

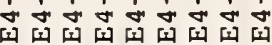

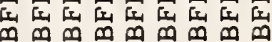




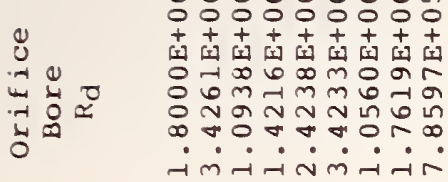

-

$\underset{1}{x}$

व

๑

$3 \frac{5}{\mathrm{c}}$

ن

ชู

漓

ช

要

का

ए.

话

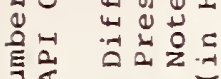

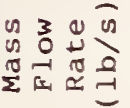

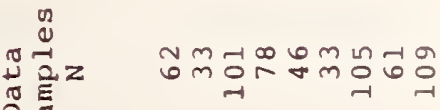

엔ำ $\ln$ mmon

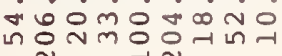

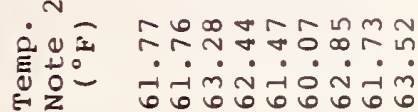

$\begin{array}{lll}0 & -1 & 0 \\ 0 & 0 & 0 \\ 0 & 0 & -1 \\ 4 & 0 & 0 \\ 0 & 0 & 0\end{array}$

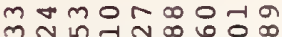

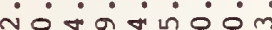
กำ

气国

है 嵌嵌

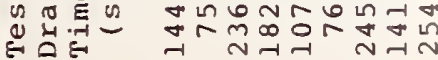

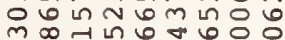

เำกำกำ $\dot{m} \dot{m} \dot{m} \dot{m} \dot{0} \dot{0}$ (u)

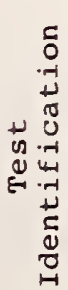

オNM П

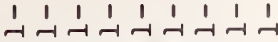

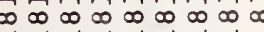

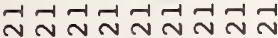
antarano 엉ㅇㅇㅇㅇㅇㅇ $\leq \leqslant \leq \leqslant \leq \leqslant \leq \leqslant$

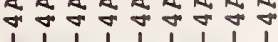

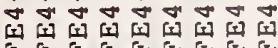

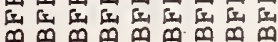
$\infty$ Nonont

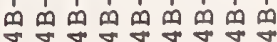

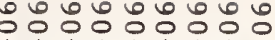

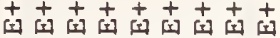

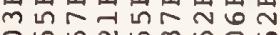

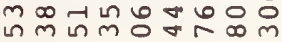

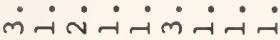

ㅁำ

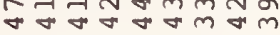

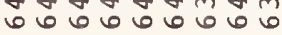

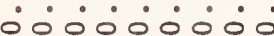

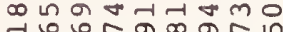
-

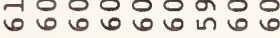
$\therefore \circ 0000000$

ㄴำ 車

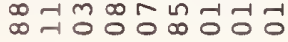
जिं்ंलिलंm

$\forall \square 6 \forall \varphi 0 m$

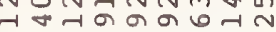

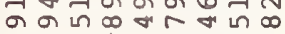
$\dot{-i m} \dot{-i} \dot{\sim} \dot{v}$

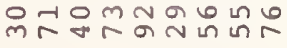

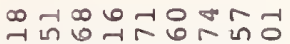
$\therefore-\dot{0} \dot{0} \infty \dot{m} \dot{\circ}$ ㄱำ

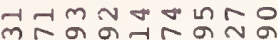
- . . . .

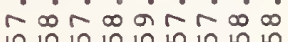

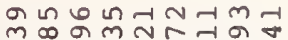
-ெं் $\dot{0} \dot{0} \dot{0}$ Mn $\infty \pi m \sim m$ 눈 성ㅇำ

nกล $\forall$ N $\forall \infty \infty \neg-m$ g은 n

이 $\dot{m} \dot{\sim} \dot{\sim} \dot{\sim} \sim \dot{\sim}$

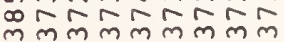

-1 ก ก $\infty \infty \infty \infty \infty \infty \infty \infty \infty$ N N N N N N N

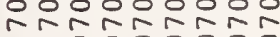
OOOOOOOOOO $\begin{array}{lllllllllll}1 & 1 & 1 & 1 & 1 & 1 & 1 & 1\end{array}$ 1 1 1111

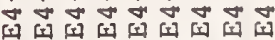

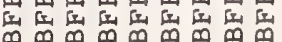

๒゚ $+t+t+t+$

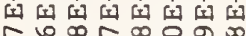
$16 \infty$ ก⿻

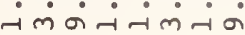

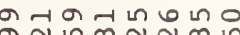
o소쇼 ชิ 060606 00000000

mmNR 0

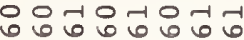
¿000000

a สNำ

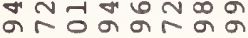
พับกัNंก

- muLnONm 는

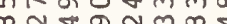

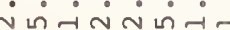

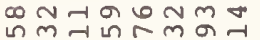

armo잉 ลิन

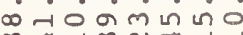

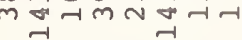

Nonmano 政 N

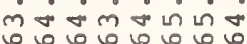

ब $\dot{0} \dot{0} \dot{0} \dot{0} \dot{\circ}$

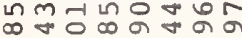
ஸี เี่

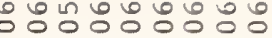

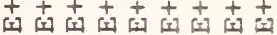
이의

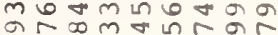

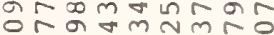
$-\rightarrow \infty \sim m-1-1$

NGน

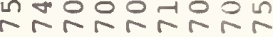
606060606 000000000

N $N \infty \infty \infty \pi-1 N$

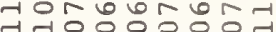
เซ 606060606 -00000000

mannt

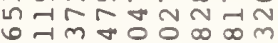

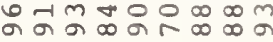
ผ $\dot{\sim} \dot{N} \dot{\sim} \dot{N}$

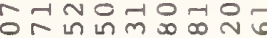
6 in 060

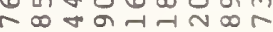
-1 - m N In N

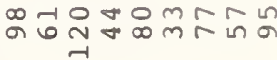

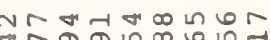

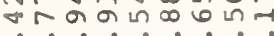

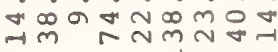

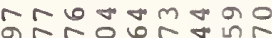
$\dot{\theta} \dot{\theta} \dot{0} \dot{0} \dot{0}$

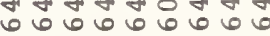

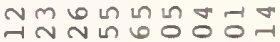
กa $\infty$ or แn เn in in in in in

ㅇำตन กMNก

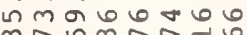
mrnmar

แีำ - $\dot{0} \dot{0} \dot{0} \dot{0}$ ก

응ำำำ

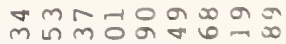
லம்பீடீடீ $\infty \infty \infty$ ô ने ने ज

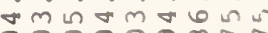
잉의의

TNm nnn $\infty \infty_{\infty} \infty \infty \infty \infty \infty^{1} \infty$ ำกำำำำ

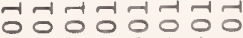

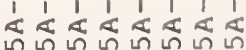
$\begin{array}{llllllll}1 & 1 & 1 & 1 & 1 & 1 & 1 & 1\end{array}$

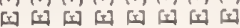

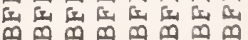

4nm ป

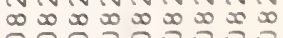

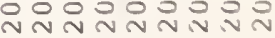

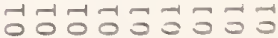
$111 x=0$

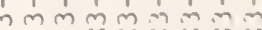

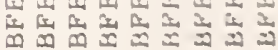

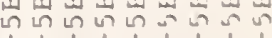




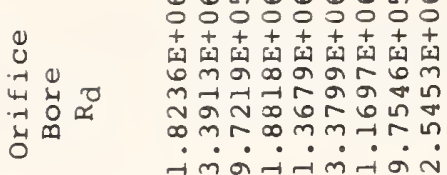

में

ro 0 a r5ingninn 6 $6060666 \%$ -

ก ก $0000 \%-1$ ง mog 0 - $N$ ن ட் $\dot{0} \dot{0} \dot{0} 0$

$04 \overrightarrow{4}$ - $10 .-4$ $4+0$

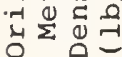

ع

วิ

0

○ـ

त)

$0+$

(1)

of

00

$\Sigma$

is

है

ह

$+0$

E⿺辶万

-

in

잉

4
2

的合嵒 的

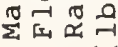

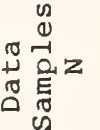

- $m \widehat{o}$

出岱曽

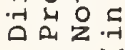

$N$

요도

E

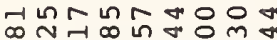

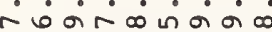

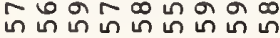

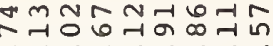

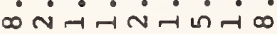

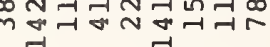

본 승ํㅇ in $\dot{\sim} \dot{\sim} \dot{\sim} \dot{\sim} \dot{-i}$

क

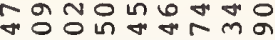
$\dot{\infty} \dot{\infty} \dot{0} \dot{m} \dot{\sigma} \dot{\sim} \dot{m}$

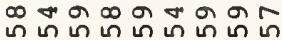

尺

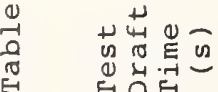

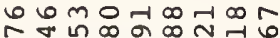

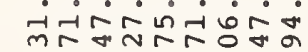
㟧 of (1)

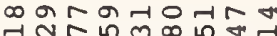
ก กับ แก ก オ

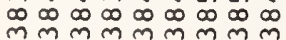

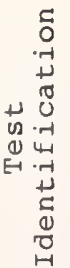

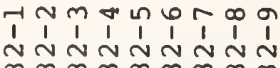

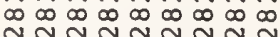

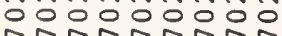

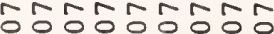
$\begin{array}{lllllllllll}1 & 1 & 1 & 1 & 1 & 1 & 1 & 1 & 1\end{array}$

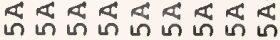

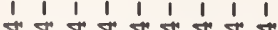

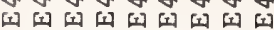

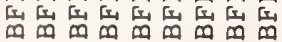

๒ย $+t+t+t+t$ 国国国国国国国国的

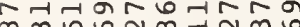
कू

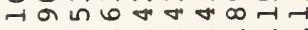
ना ब N

맘ㅇㅇㅛ

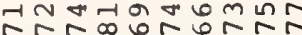
6060600606 ○ं $0 \dot{0} 00000$

N œ

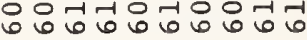

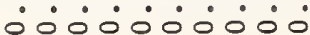

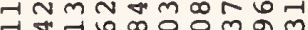
ตํํㅇㅇㅇำ

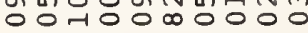
$\dot{m} \dot{m} \dot{m} \dot{m} \dot{\sim} \dot{m} \dot{m}$

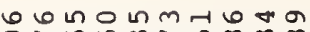
ำ

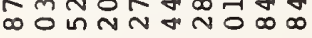
$\dot{-i \dot{r}} \dot{\sim} \dot{\sim} \dot{\sim} \dot{m} \dot{-} \dot{-}$

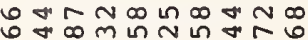

NNDMMOOnM

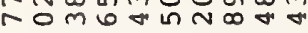
ம்ல்

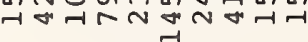

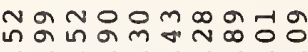
añ $\infty \dot{\infty} \infty \sigma^{\circ}$ in in in in in in in in in in

ำ

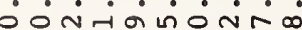
七ด

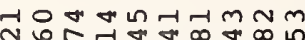
ㄴ.? ñ बำ

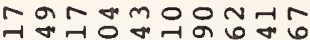
$\dot{0} \dot{0} \dot{0} \dot{\infty} \dot{\infty} \dot{0} \dot{0}$ $\sim \infty \infty \infty \infty \infty \infty$ a minmm

अ彳 ง่ำ $\infty \infty_{\infty} \infty \infty_{\infty} \infty \infty_{\infty} \infty \infty_{\infty} \infty \infty^{\infty} \infty$

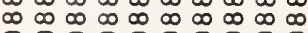
○ 000000 rrorkr 잉ㅇㅇㅇㅇㅇㅇㅣ $\begin{array}{llllllllll}1 & 1 & 1 & 1 & 1 & 1 & 1 & 1 & 1 & 1\end{array}$

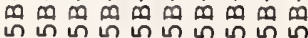
$\begin{array}{lllllllllllll}1 & 1 & 1 & 1 & 1 & 1 & 1 & 1 & 1 & 1\end{array}$

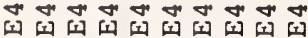

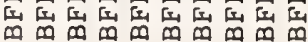

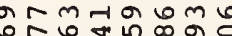
?.? * o in $\infty$ ina *กลก

๒ $\dot{0} \dot{0} \dot{\infty} \dot{\infty} \dot{\infty}$

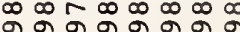
$\mathrm{mm} \mathrm{mm} \mathrm{m}$

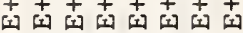
OmNRT $\infty \infty m b \neq \sigma \omega$ の ก

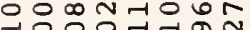

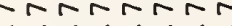

Gonmonnm 동ํㅇㅇㅇㅇㅇㅇ $\therefore \circ \circ \circ \circ \dot{0}$

mon $\operatorname{con} \sigma$

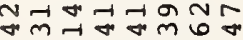
aर बक क

ㅎำ

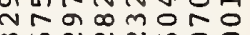
ก.? แก $\sim$ เก

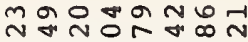

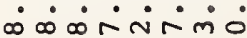

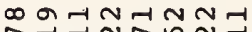
$\therefore \dot{0} \dot{0} \dot{0} \dot{0}$

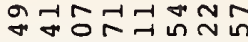

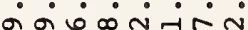
约 n in

m+in or o o

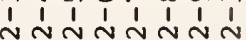
$\infty \infty \infty \infty \infty \infty \infty$ ำสำสำสำส

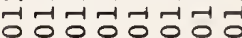
$\begin{array}{llllllllll}1 & 1 & 1 & 1 & 1 & 1 & 1 & 1\end{array}$

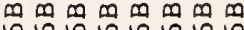

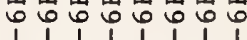
$m m m m m m$

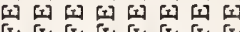

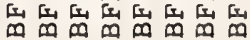

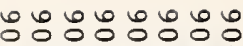
nกNำกำ

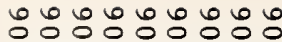
$++t++++t$ 两向60 $m \infty$ 我 $\infty m \infty \infty$ $\sigma \infty \sim \infty$ in $\rightarrow m$ ง $\rightarrow-1 \sim-1-1$

nर Nํำกำกำก nNhNnTNNA $\dot{\circ} \dot{0} \dot{0} \dot{0} \dot{0} \dot{0}$

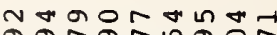
ㅇํㅇํㅇㅇํㅇํㅇ응 ○ं $0 \dot{0} \dot{0} \dot{0} 0$

뭉요 बNํำ - ă ă áă ó $\dot{\sim} \dot{N} \dot{\sim} \dot{N} \dot{N} \dot{N}$

ก $m$ n $N$ mon

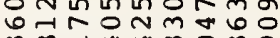
$m-\forall \infty \circ m \sigma$ $\dot{m} \dot{\sim} \dot{m} \dot{\sim} \dot{\sim} \dot{\sim}$

나ำㅇำ

그유 กㄴ.

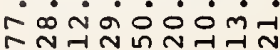

Taษ แก $\rightarrow 6$ แn un un $\forall-$

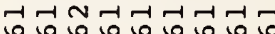

ํํㅇㅇㅇㅇㅇㅇㅇำ $\dot{0} \dot{0} \dot{m} \dot{-1} \dot{0} \dot{r}$

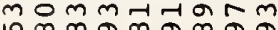
นกํำ ติ

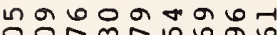
$\dot{0} \dot{0} \dot{-} \dot{0} \dot{0}$ ०

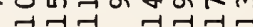

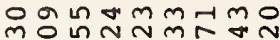
نூ

1

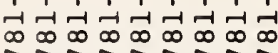
rarasoma

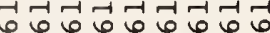
000000000 $\begin{array}{lllllllll}1 & 1 & 1 & 1 & 1 & 1 & 1 & 1 & 1\end{array}$

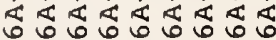
1 1 1 1111111

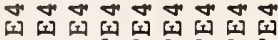

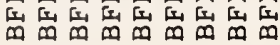




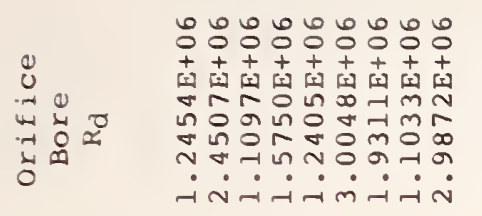

-

* $\quad$ nก

$\dot{0} \dot{0} \dot{0} \dot{0} \dot{0} \dot{0}$

 กตกตำนก

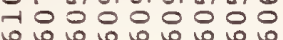

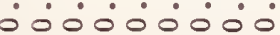

\section{0 us \\ กำ-1 9 क N}

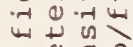
ज्ञाँ

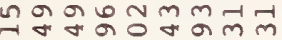

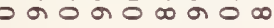
$\dot{m} \dot{m} \dot{\sim} \dot{\sim} \dot{\sim} \dot{N}$

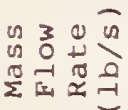

onR

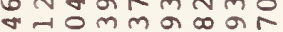
volnm ข $\dot{\sim} \sim \dot{\sim}$ ம்

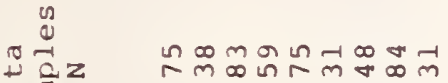
ดกต์ نं $\dot{0} \dot{0} \dot{0}$

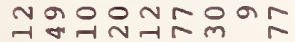

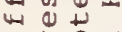

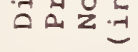

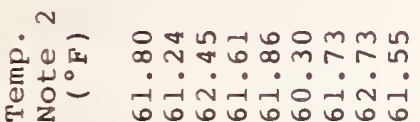

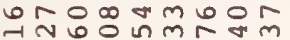
$\dot{0} \dot{0} \dot{0} \dot{0}$ 는

mูㄷํㅇㅇㅠ

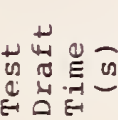
matom

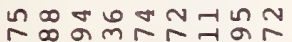

우유. $+++++++++$

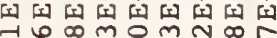

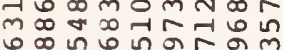
๒ำง -ன் $\dot{-1} \dot{\boldsymbol{N}} \dot{-} \dot{\mathrm{N}}$

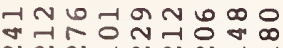
nักำ $\circ \dot{0} \dot{0} \dot{\circ} \dot{0}$

๒ 겅ำ ○ं0ல் 00

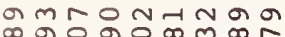

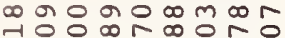

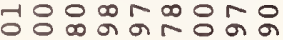
$\dot{m} \dot{\sim} \dot{\sim} \dot{\sim} \dot{m} \dot{N}$

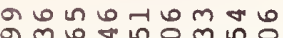
$\infty-\pi m-\infty \infty \infty$ ०m Nं $\dot{\sim} \dot{\sim} \dot{\sim} \dot{N} \dot{\leftarrow}$

mㅇํำ

mㄴำ $\dot{0} \dot{0} \dot{0} \dot{0} \dot{0} \dot{0}$

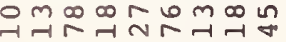

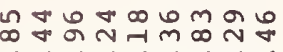
$\dot{\infty} \dot{\infty} \dot{0} \dot{\sim} \dot{\sim} \dot{\infty} \dot{\infty} \dot{r}$ ก

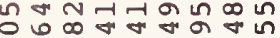
$\dot{\sim} \dot{\sim} \dot{\infty} \dot{m} \dot{-1} \dot{-1}$ กิกี่

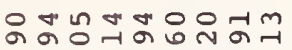
$\therefore \circ \dot{0}-10$ เ

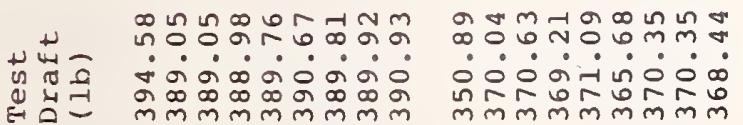

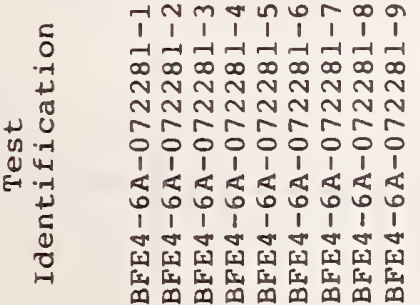

Tกตำ

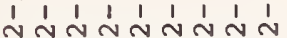
$\infty \infty \infty \infty \infty \infty \infty$ $\infty \infty_{\infty}^{\infty} \infty \infty_{\infty}^{\infty} \infty \infty \infty \infty$

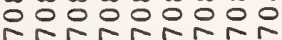

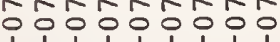

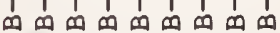

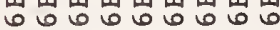

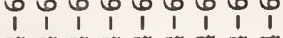

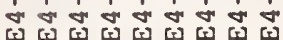

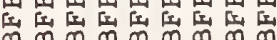


Table $8(a)$. Test Meter Identification and Physical Measurements Nominal 4 Inch AGA/API Orifice Meter (British Units).

Test

Identification

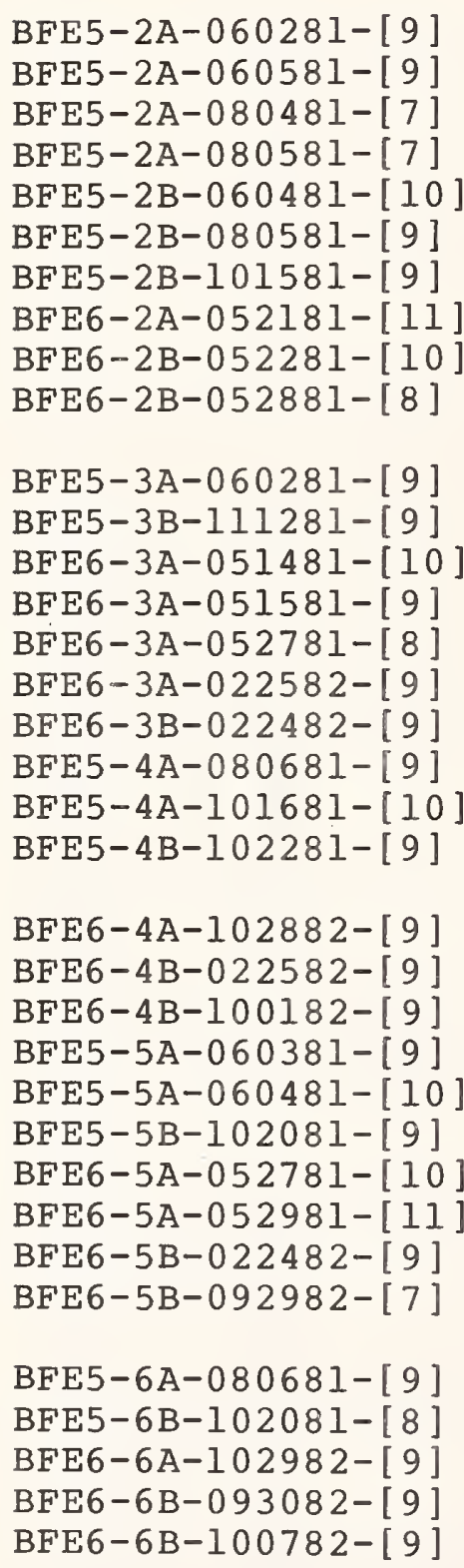

Test

Configuration
Run

Diameter

(inches)
Orifice

Diameter

(inches)

1.4993

$$
\begin{aligned}
& 1981-A \\
& 1981-A \\
& 1981-A \\
& 1981-A \\
& 1981-A \\
& 1981-A \\
& 1981-A \\
& 1981-A \\
& 1981-A \\
& 1981-A
\end{aligned}
$$

$1981-A$

$1981-A$

$1981-A$

$1981-A$

1981-A

1981-A

1981-A

1981-A

1981-A

1981-A

1981-A

1981-A

1981-A

1981-A

1981-A

1981-A

1981-A

1981-A

1981-A

1981-A

1981-A

1981-A

1981-A

1981-A

1981-A

4.0223

4.0223

4.0223

4.0223

4.0223

4.0223

4.0223

4.0260

4.0260

4.0260

4.0223

4.0223

4.0260

4.0260

4. 0260

4.0260

4.0260

4.0223

4.0223

4.0223

4.0260

4.0260

4.0260

4. 0223

4. 0223

4.0223

4.0260

4.0260

4.0260

4. 0260

4.0223

4.0223

4.0260

4.0260

4.0260
1. 4993

1.4993

1.4993

1.4996

1.4996

1.4996

1.4993

1. 4996

1.4996

1.9990

1.9992

1.9990

1.9990

1.9990

1.9990

1.9992

2. 2491

2. 2491

2. 2495

2. 2491

2. 2495

2. 2495

2. 6244

2. 6244

2.6248

2. 6244

2. 6244

2. 6248

2. 6248

3.0001

2. 9993

3.0001

2.9993

2. 9993
Beta

Ratio

0.3728

0.3728

0.3728

0.3728

0.3728

0.3728

0.3728

0.3724

0.3725

0.3725

0.4970

0.4970

0.4965

0.4965

0.4965

0.4965

0.4966

0.5592

0.5592

0.5593

0.5586

0.5587

0.5587

0.6525

0.6525

0.6526

0.6519

0.6519

0.6520

0.6520

0.7459

0.7457 .

0.7452

0.7450

0.7450

Note: Test Identification bracket gives number of tests run with this geometry. A total of 316 tests were run on the nominal 4 inch run size. 


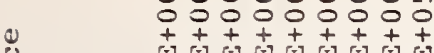

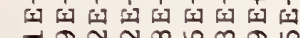
แ山 का

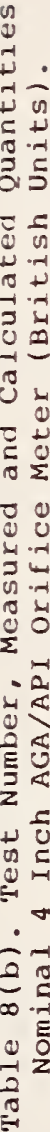

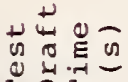

บก N $\infty$ N

iन ส

U) ज नं

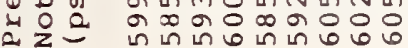

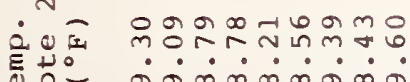

E

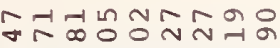

능ํํㅇำก

.

ब००नान100

U. N N

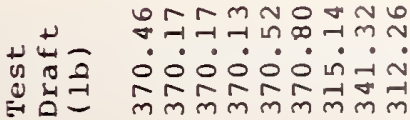

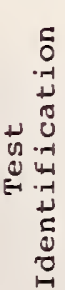

INm

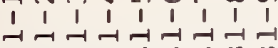
$\infty \infty^{\infty} \infty \infty^{\infty} \infty \infty^{\infty} \infty \infty^{\infty} \infty$ Nก ก้ ก้ก กิก 00060060606 $\because 0: 000000$

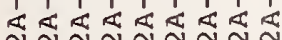
4⿻ ก ᄂ

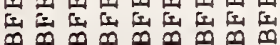

ஜ๐๐요 $++++\begin{aligned} & + \\ & + \\ & +1\end{aligned}++$

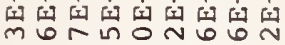
$\infty \pi$ r $m$ m का न

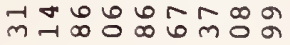
-i்i $\dot{\sim} \dot{-i} \dot{\sim} \dot{0}$

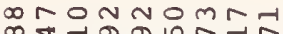

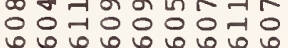
$\therefore \circ \circ \circ \circ \circ \circ \circ$

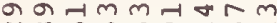
ํํㅇำ

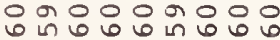
ல் $\dot{0} \dot{0} \dot{0} 0$

NNmTNRTR Nํำก ํํำ लंखं $\dot{\sim m} \dot{\sim}$

N N $N M \infty \operatorname{moO}$ $-15 \infty 0 \pi$ 元

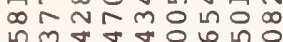
$\dot{-i} \dot{\sim} \dot{\sim} \dot{\sim} \dot{\sim} \dot{\sim}$

ㄴํㅇำ 각

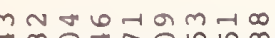

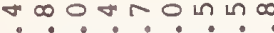

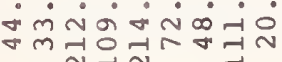

ดัตกน - $\cdot \cdot \cdot \cdot \cdot \cdot \cdot$

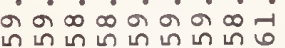

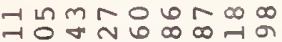
- $\dot{\sim} \dot{\sim} \dot{\sim} \dot{0} \dot{0} \dot{0}$ ํํㅇํำ

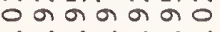

은 ?. . . .

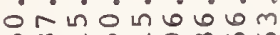
เึำ

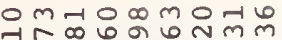

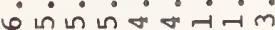
ने बून नून लm m m लm m m

-1 $⿻$ m

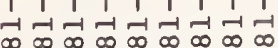

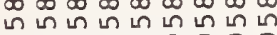

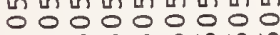

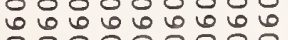
000000000

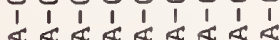
ฟ

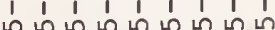

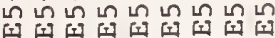

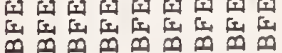

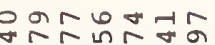

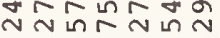
MNNNNN

№-1ma बึ่ -iिंنं $\dot{\sim} \dot{-i}$

능응요

음aำ o.? inं ก.?.? a

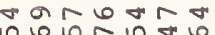

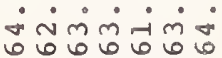

응 으멘 $\dot{0} \dot{\dot{m} \dot{m}} \dot{\sim} \dot{\sim}$ 6666060

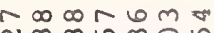
N $\infty \infty 6 \mathrm{mo}$ $\dot{m} \dot{-1} \sim \dot{0} \dot{0}$

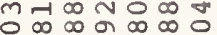
용ำ

เป็ மं $\dot{m} \dot{\sigma} \dot{\sigma}$ a $0 \infty \infty$

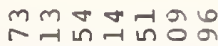
बNल NmंNm

แกำ कิतन 1ึ $\therefore \dot{-1} \dot{\sim} \dot{-i}-$

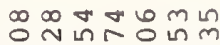
in 0 in 0 1 6

ดNN ำ $\dot{\sim} \dot{\sim} \dot{0} \dot{\sim} \dot{1}$

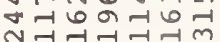

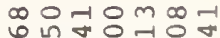
$\dot{0} \dot{\sim} \dot{0} \div$

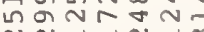

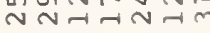

ำ ำㅇํำ

ด ต ०र००नक न

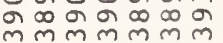
० $m m+\infty$

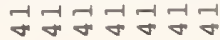

I1 -1 1 - 1 -

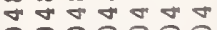
0000000

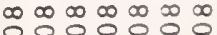

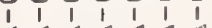
岕芯爬芯 $\begin{array}{lllllll}1 & 1 & 1 & 1 & 1 & 1 & 1 \\ & & & & & & \end{array}$

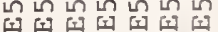

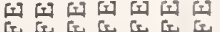

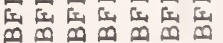

TNM는 का กึกนก็นก 엉요 ○ 000000 สิ I I I I 1 1 11

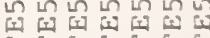

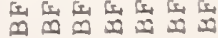




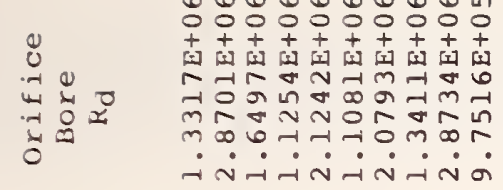

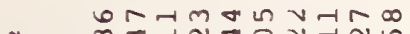

$x^{2}$

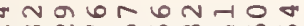
Nonnแ

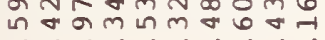
$\rightarrow$ m $-\dot{\sim} \sim \dot{-1}$ 范

ำกำติ

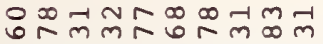
- . . . . . mo

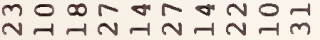
舟こ

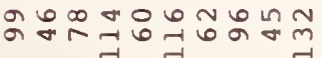

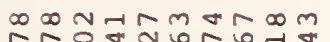
-

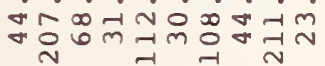

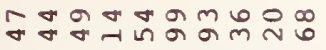

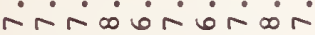
in in in in in in in in in in

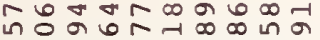
- $\dot{0} \dot{m} \dot{-i} \dot{0} \dot{\sim} \dot{\sim}$ ลิ * $\mathrm{mm} \mathrm{mm} \mathrm{mm} \mathrm{m}$

$\begin{array}{lllllllllll}1 & 1 & 1 & 1 & 1 & 1 & 1 & 1 & 1 & 1\end{array}$

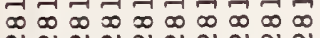
N $N \sim \sim N \sim N \sim N$ NกNกNกNกNก

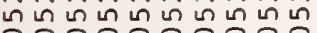
OO 00000000

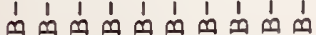

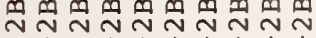

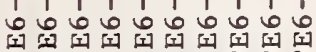

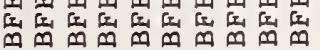

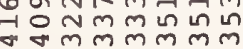

๕๐ะேะேะே

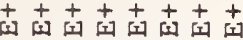

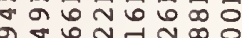
๙ิ

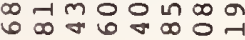
-iिम-ं $\dot{\sim} \dot{\sim}$

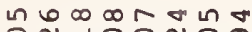

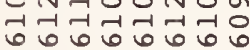
0

6r $\infty$ an $\infty$ in テ๒ 00000 ல0்0ல0்

윰ำ

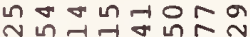
กิ $\dot{m} \dot{\sim} \dot{m} \dot{\sim} \dot{N} \dot{~}$

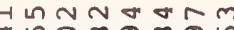

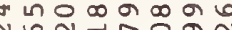
N ?m vंगनंतिं

$\infty$ 네뭉

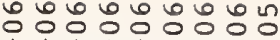
$++t+t+t+t$

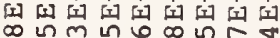

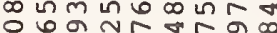
公。 $\infty-\cdots \infty \neq m+$ $\dot{-} \dot{\sim} \dot{\sim} \dot{\sim} \dot{m} \dot{-} \dot{0}$

$\infty \infty \backsim \infty \sigma a \infty \sim$ ฟNNNNNNํำ

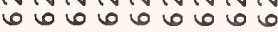
00000.0.0

n $\ln$ in 0 w n 00 nก $m$ in $m$ in $N m \infty$ 응ㅇㅇㅇㅇㅇㅇㅇㅇㅇㅇㅇㅇㅇ

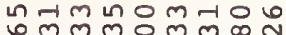
ำ 응ํㅇㅇㅇㅠ mं் $\dot{n} \dot{m} \dot{\sim} \dot{m}$

*

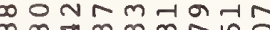

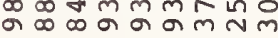
vim $-\dot{m}$ i

ตำตำกำ

แกำกー ขन Nं்่ $\dot{\sim} \dot{0} \dot{-1}$ ㅇำ

ตฺฺ ด.? ก ? $\infty \infty \infty \infty \sim \infty$ a แn เก แก แn แก เก แก เก

누의의 $\dot{0} \dot{0} \dot{0} \dot{0} \dot{0}$

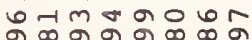

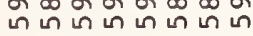

ก⿻上Anำก ช 6 กับ n-

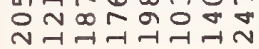

a $\infty$ ON ำ o

-1

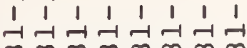

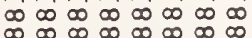

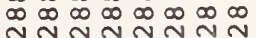

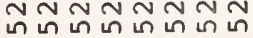

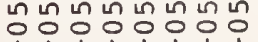

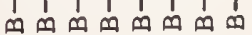

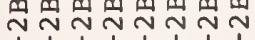
1

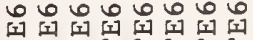

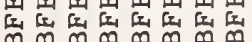

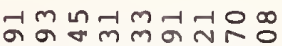
$\therefore \infty 00$ ல $\forall-\infty N+\infty$ d

$m b 0$ a $1 m$ แn $N$ ? $\infty$ ? ? ? a $\infty \sigma \infty \infty \sigma a$

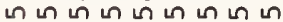

ㄱํ유 హె $\dot{m} \dot{\circ} \dot{\sim} \dot{-1} \dot{0} \dot{\infty} \dot{0}$ ํำ

언요은 $m$ ก ๑- निं

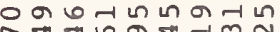
-i்லن்-i் $\dot{\sim} \dot{m}$ लَñ

HNm n on

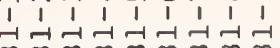

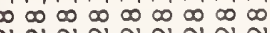

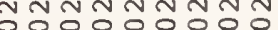
060609606 0 0000000

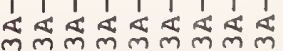

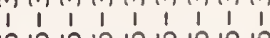
ถ

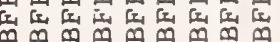
○ं0ல் 0000

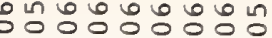
$++++++++t$ न न $\sigma^{\infty} \pi \sigma^{\infty}$ in 0 in

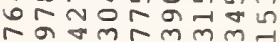
- $\dot{\sim} \dot{\sim} \dot{-1} \dot{\sim} \dot{-\infty}$

กแกนำบํำ

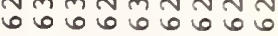
000000000

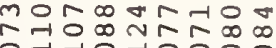
입 ○ं0ல0ல000

이이의

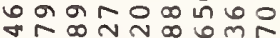

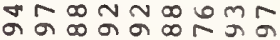

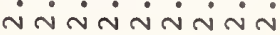

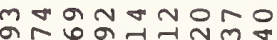

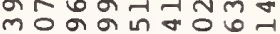
$\infty 0 \infty 0 \infty \infty m$ N $\rightarrow$ m N $N$ In $N$

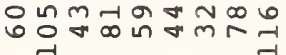

๙

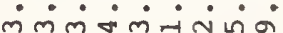
m $m$ m

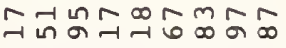
ก กm ด

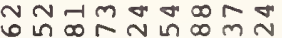
$\dot{0} \dot{0} \dot{\sim} \dot{0} \dot{0} \dot{0}$ $\infty$ นn

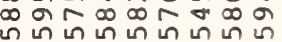
mำ ก.?. $\dot{0}-\dot{0} \infty \dot{\sim} \sim$ $m=0 \infty m 0 N$

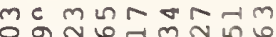
$\dot{0} \dot{0} \dot{0} \dot{0} \dot{0} \dot{0}$

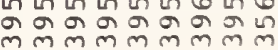

TNm

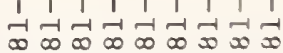

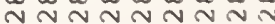

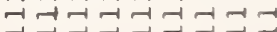

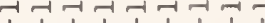
$\infty \infty \infty \infty \infty \infty \infty$ । ।

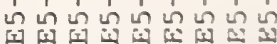

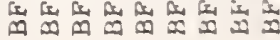




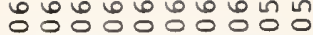
0 $\begin{array}{lll}-1 & 0 \\ 4 & 4 \\ -1 & 0 \\ -1 & 0 \\ 0 & 0\end{array}$ $++++++++++$ $\infty$ जिa

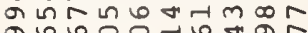
बढ़ $\dot{\sim} \dot{-i} \dot{-} \dot{\sim} \dot{\sim} \dot{\sim} \dot{\sim}$

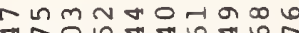
בัy

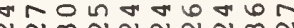
ฟิ ல் $\dot{0} \dot{0} 0 \dot{0} 0$

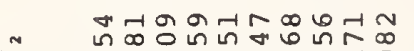

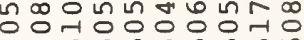
$\therefore \circ \dot{0} \dot{0} \dot{0} \dot{0}$

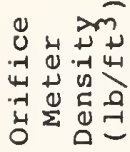

กี

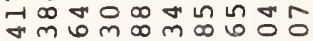
" नूँ

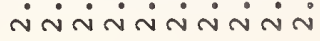

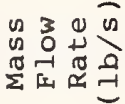

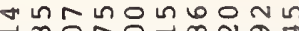
ㄱํㅇㅇำ 约 $\dot{\sim} \dot{\sim} \dot{\sim} \dot{\sim} \dot{+} \dot{-} \dot{-}$ 竞点

음ำ - mo

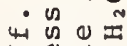
$40+$ 年出员.

momunmato mañ نே

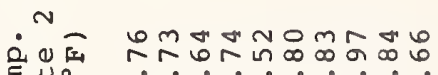

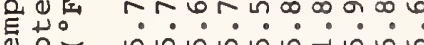
Е

$\dot{\theta}^{-1}$ क

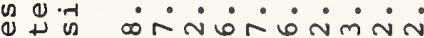

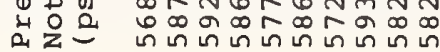

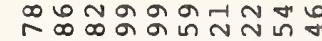

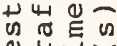

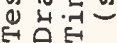

㻤㟧

-

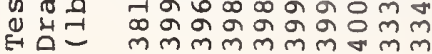

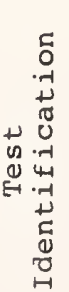

Tขm

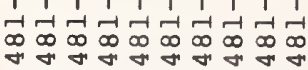

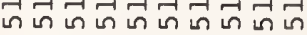

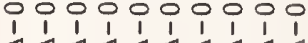

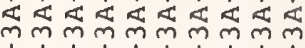
$\begin{array}{llllllllll}1 & 1 & 1 & 1 & 1 & 1 & 1 & 1 & 1 & 1\end{array}$

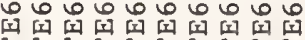

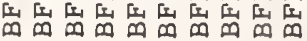

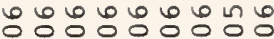

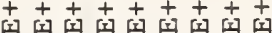
ง

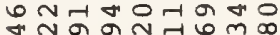

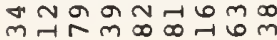
$-\dot{-i} \dot{-i} \dot{i} \dot{-i} \sim$

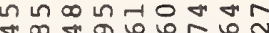

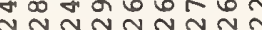

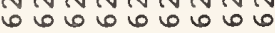
லட் $0 \dot{0} 0 \dot{0}$

NH约เึ็นกำ 웅은 606060060 ¿0ं0்00்0

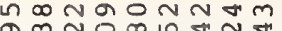

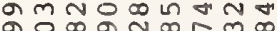

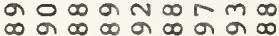
ํำ่ำ่

ก) $m \infty m \infty \backsim n \infty$

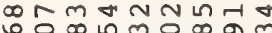
-

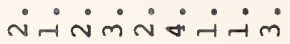

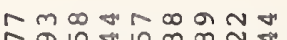
$\therefore \sigma$ 皮

$\infty m \sim 6$ ○ 0 m $\infty$

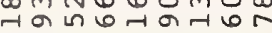
or.

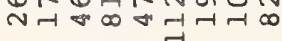

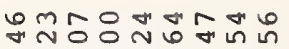
$\dot{0} \dot{0} \dot{0} \dot{0} \dot{0} \dot{0}$

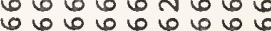

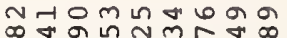
$\dot{\circ} \dot{0} \dot{\infty} \dot{m} \dot{m} \dot{r}$

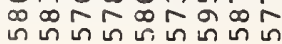

부임 กํ. №ñmon i N

ํํำกำกำ

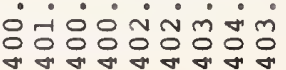

Nm

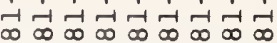

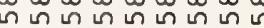
กี่ 0 O 0000000

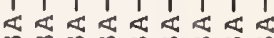
1 b 601000010

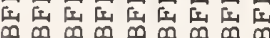

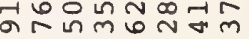
तें் $\dot{m} \dot{m} \dot{m}$

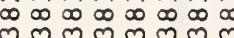

붕요융

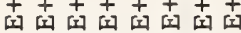
N

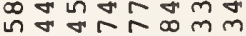
$\rightarrow m \infty \forall m-1$ -imनंत्ना

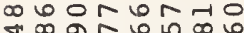
ขกง ๑ 6 6 $606 \omega$ 00000000

nhnthan

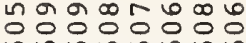
. . . . ○ं 00000

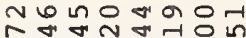

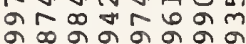
$\dot{\sim} \sim \dot{\sim} \dot{\sim} \dot{\sim}$

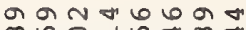
$\infty$ ᄂกㄱำ

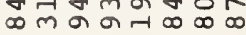
-i

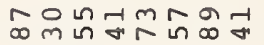

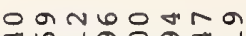
.

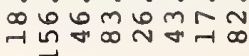

잉ำ - m.

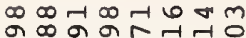
$\dot{0} \dot{0} \dot{0} \dot{0} \dot{0}$

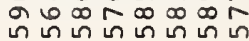

Oดm ín

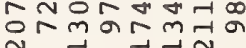

$-1-1-1-1-1-1-11-1$ $\infty$

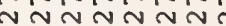

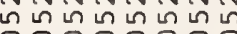
O O O OOOOO

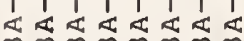

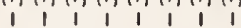
o lo 000100

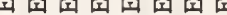

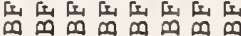

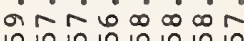

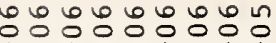
$++t+t+t+t+t$ o

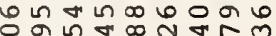
ฌ -i் $\dot{m} \dot{\sim} \dot{-i} \dot{\sim}$

แ

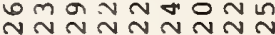

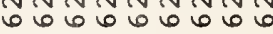
○ंல் 0000

No

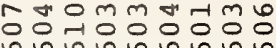
$\therefore \circ \circ \circ \circ \circ \circ \circ \circ$

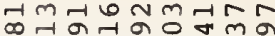
oradotañ

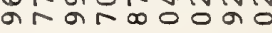
Nंن்่ $\dot{\sim} \dot{m} \dot{\sim}$

$6 \mathrm{~m}$ in 9 요은 m० न न क न

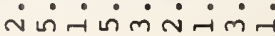

ঢีm ก

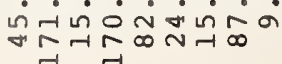

monatanta 6m -

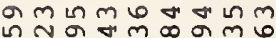
$\dot{0} \dot{i} \dot{m} \dot{\mathrm{m}} \dot{0} \dot{0}$

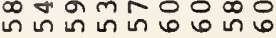

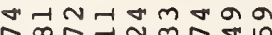
- $\cdot$.

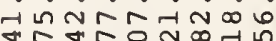
$\rightarrow$ 슴ำ

ส ทำ-

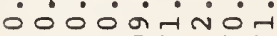

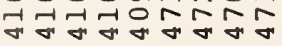

TNm ป

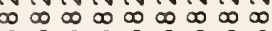

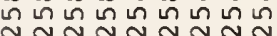
NNNNNNNN 0000000000

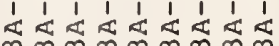
$\begin{array}{lllllllll}1 & 1 & 1 & 1 & 1 & 1 & 1 & 1 & 1\end{array}$ ๑ 00060000

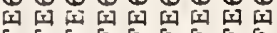

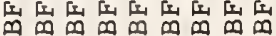


N

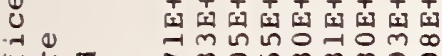
mo mmea

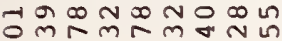

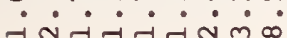
ก $\quad$ ํำ

শ્ર

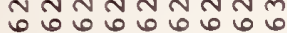
○ं00000000

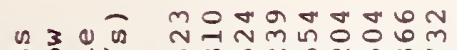

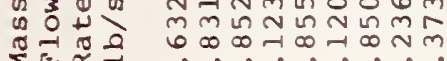
こ

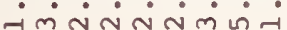
踏造 - in 4 is $4 x$

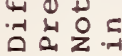

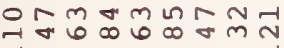

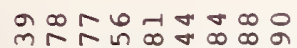
ปั

这这

MMNODNGO

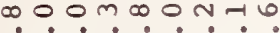

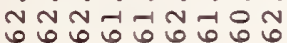

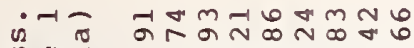
(2) प0

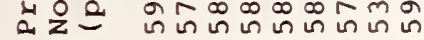

ㅇํㅇำ Lกำ นำㅇำ

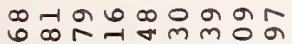

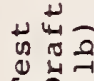

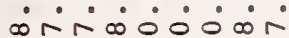
药

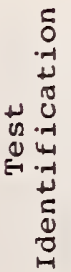

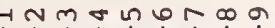

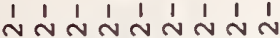
$\infty \infty_{\infty}^{\infty} \infty \infty_{\infty}^{\infty} \infty \infty_{\infty}^{\infty} \infty \infty_{\infty}^{\infty} \infty$

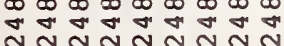
NN NNNNNN i 00000000

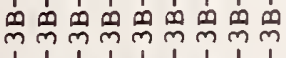
$\begin{array}{lllllllll}1 & 1 & 1 & 1 & 1 & 1 & 1 & 1 & 1 \\ 0 & 0 & 0 & 0 & 0 & 0 & 0 & 0 & 0\end{array}$

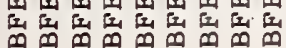

ㅇํํㅇํㅇำ

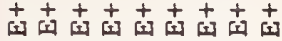
o on $\infty$ in 0 r

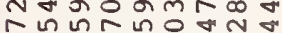
กำलंनंतंतंतं

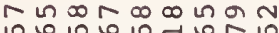
in

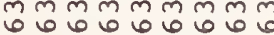
ல0் 00000

ตำ ले 으웅요용요웅 $000000000^{\circ}$

* NUน

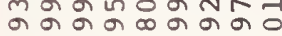

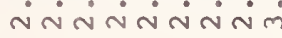

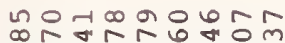
कर

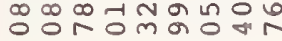
*NAmintan

기요

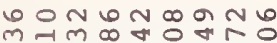
i $\dot{0} \dot{\circ} \dot{\infty} \dot{\sim} \dot{0}$

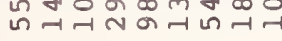

HNDm 0 -

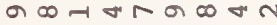

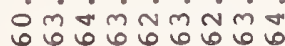

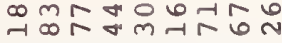
- irina-io

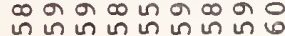

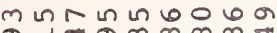
-

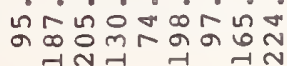

ㄱํำ ن் $\dot{0} \dot{0} \dot{0} \dot{0} \dot{0}$

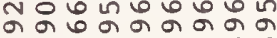

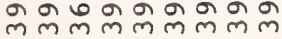

-1 2 m

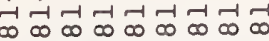
0 0006006 $\circ 00$ œ

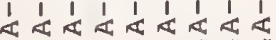

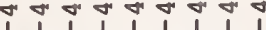

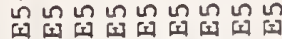

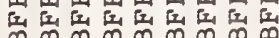

ํํㅇํำำำ

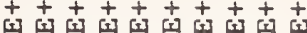

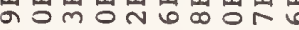

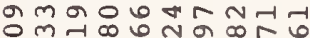
응ㄱำ mंன் $\dot{\sim} \dot{\sim} \dot{\sim} \dot{\sim}$

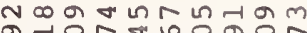

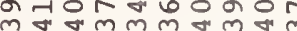

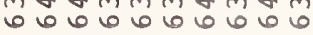
0000000000

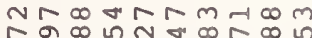

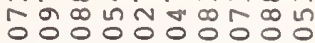

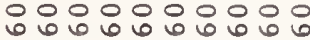
0ं0000000்

-10n $\infty$ o

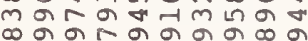
iั $\dot{\sim} \sim \dot{N} \sim \dot{N}$

NmLONaan

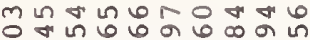

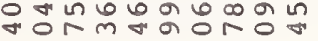
แก

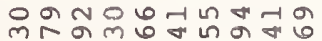
ตำำก는 a ma

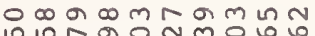
- $\dot{0} \cdot \dot{0} \dot{0}$

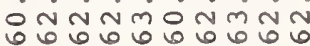

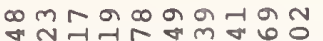

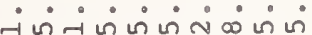

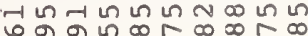

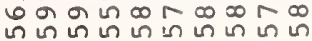

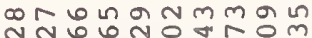
$\dot{0} \dot{0} \dot{0} \dot{0} \dot{0} \dot{0} \dot{0}$

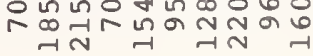

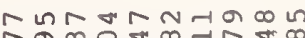
ก.?

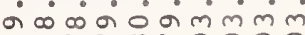

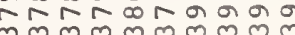

กงm母n

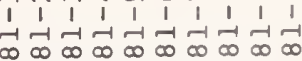

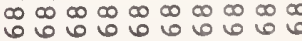

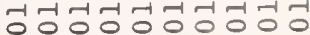
ન્1

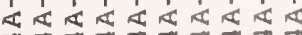

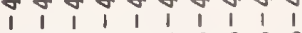

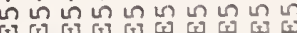

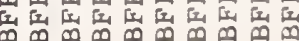

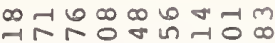
लंखन

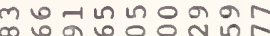
$\dot{0} \dot{0} \dot{0} \dot{0} \dot{0}$ ติ

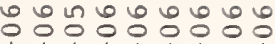

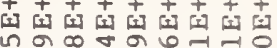
$\forall-1 m m$ in สㅇำ

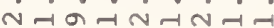

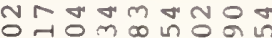
000000000

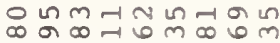
ㅇํㅇํำ லं0ல்0000

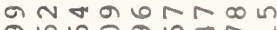
누의

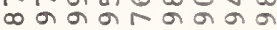
กักกักกก

નี ब न $m-1 m \backsim-\mathbb{N}-$

สำ ำกำ

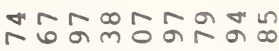
$\dot{m} \dot{\sim} \dot{\omega} \dot{\sim} \dot{\omega} \dot{m}$

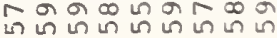

অำ $\therefore \dot{0} \dot{0} \wedge \dot{0} \dot{0}$

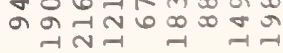

D. काก) orir no no

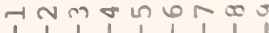

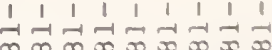
$\infty \infty^{\infty} \infty \sim \infty$ ก ก ก N OOOOOOOOO

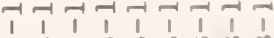

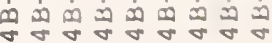
$\begin{array}{lllllll}1 & 1 & 1 & 1 & 1 & 1\end{array}$

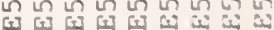

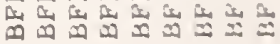

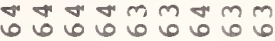




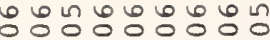

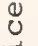

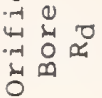

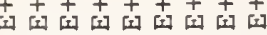
बूल N $\infty \forall \infty a r m+i a$

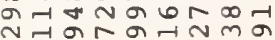
Nंबंतं $\dot{\sim} \dot{\sim}$

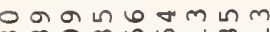

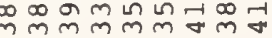

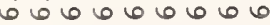
00000000

Hoanmam bratmmala U

8 ำ

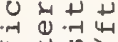

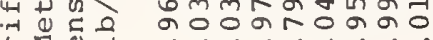
州芒

NmलN

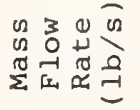

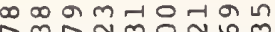
ก

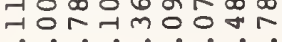
$\dot{\sim} \dot{\sim} \dot{m} \dot{\sim} \dot{\sim} \dot{\sim}$

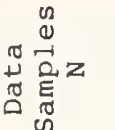
๙ุด

- mo

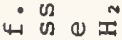
世

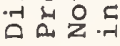

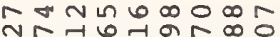
نं

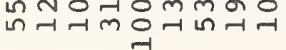

N

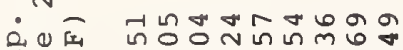
ํํำ Eั0

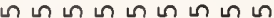

थ 0.7 $0+0$ क्ष 0 2

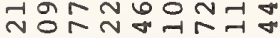
$\dot{0} \dot{0} \dot{0} \dot{0} \dot{0} \dot{-}$

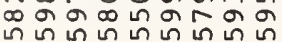

Q

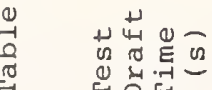

ำกำ ก้กตั ก.ำ. ก ก

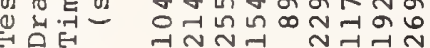

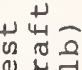

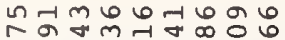

(1) 4 든

$\dot{0} \dot{0} \dot{0} \dot{0} \dot{0}$

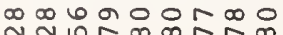

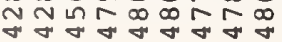

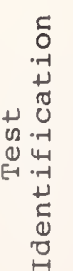

-1 1 m ป่ $\infty \infty \infty \infty \infty \infty \infty \infty$ $\infty \infty \infty \infty \infty \infty \infty \infty$ N N N N N N N N ○ 00000000

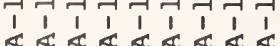

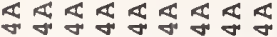
$\begin{array}{lllllllll}1 & 1 & 1 & 1 & 1 & 1 & 1 & 1 & 1\end{array}$ 国囿国

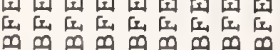

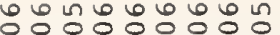
$++++++++t_{101}^{+}+$ N几心 $m \sigma \infty m \sigma=m \infty-1$

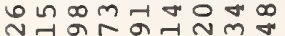
vंबंत्रिं

ก

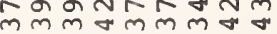

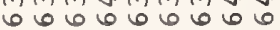
¿0000000

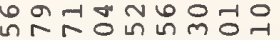

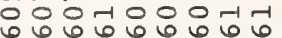
- 0000000

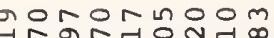
mㅇํㅇ

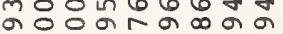
ขm $\sim \dot{\sim} \sim \mathcal{N}$

a 6 DNONRON

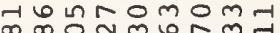
ฌ॰ $\dot{\sim} \dot{\sim} \dot{m} \dot{N} \dot{m} \dot{\sim}$

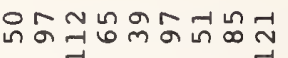

$\circ \infty N$ m $996 m$ $\therefore \dot{0} \dot{0} \dot{0} \dot{0} \dot{0}$ जननल लून हीन

ำก-19ma กำ ஸ் $\dot{\sim} \dot{\sim} \dot{\sim} \dot{\sim} \dot{\sim}$ ० 606 in 6066

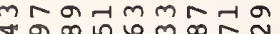
$\dot{0} \dot{0} \dot{0} \dot{0} \dot{0}$ $\infty \sigma \infty \infty \approx \infty \infty$

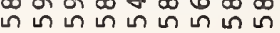

ดก $N m \circ m \infty N$ ช बm एก ก ก N

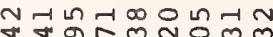
- -100.00

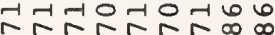

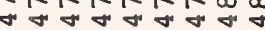

Tnm ปั่ $\infty \infty \infty \infty \infty \infty \infty \infty$

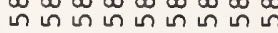
ก ก ก บ N N $N \mathcal{N} N$ N N O00000000

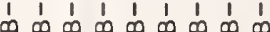

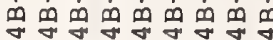
$\begin{array}{lllllllll}1 & 1 & 1 & 1 & 1 & 1 & 1 & 1 & 1\end{array}$ 66060

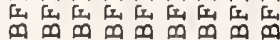

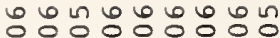
$+t+t+t+t$

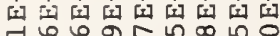
红。 in

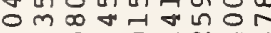
mनIñOn

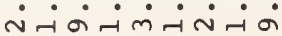

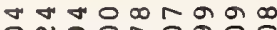
구의

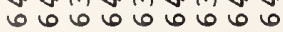
0000000.0

ฟNํㅇㅇำ

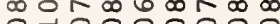

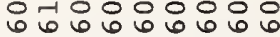

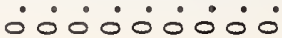

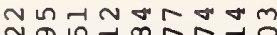

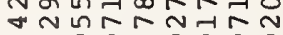
สํํํํํํำ نंखिं $\dot{\sim} \sim \dot{\sim}$

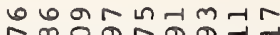

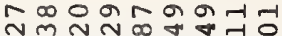

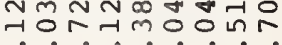
$\dot{\sim} \dot{\sim} \dot{m} \dot{\sim} \dot{\sim} \dot{\sim}$

ஆ

ซัำنिं் -ं் แn

HonNGGR

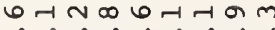

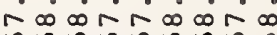
in in เी in in แn in in in

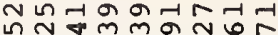

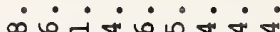
กำ

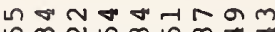
แก Norindo

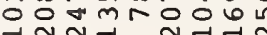

กิN $\cdot \dot{0} \dot{0} \dot{0} \dot{0}$

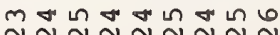

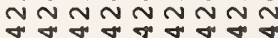

ศกm ค ป่ $\infty \infty \infty \infty \infty \infty \infty \infty$ 겅ㄷㅇㅇㅇㅇㅇㅇㅇㅇㅇㅇㅇㅇㅇㅇㅇㅇㅇㅇㅇ

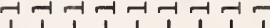
$\infty 00000$

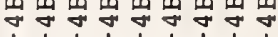

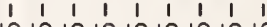
제 [1

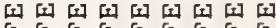

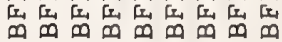

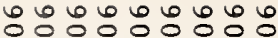
$+++++++$ ०6न- 6 б 궁

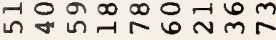
$\dot{-i} \dot{\sim} \dot{i} \dot{i} \dot{-i}$

늠ำ nNañ 666666666 ○ं 000000

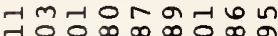
नु00000000

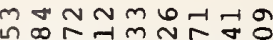
-1 ○ढ口 mivi $\dot{\sim} \dot{\sim} \dot{\sim}$

-10nNmNan

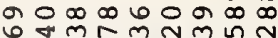

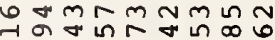
$\dot{m} \dot{\sim} \dot{\sim} \dot{m} \dot{\sim} \dot{\sim} \dot{m}$

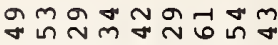

ๆ

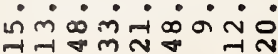

mon $\infty N \infty N$

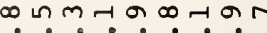
$\infty \infty \dot{0} \dot{0} \dot{\sigma} \dot{r}$

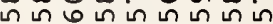

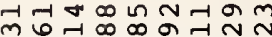
$\dot{\sim} \dot{\dot{r}} \dot{0} \dot{-} \dot{\sim} \dot{0}$ ब

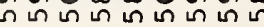

든ำ ก ᄂ $\infty$ \& $\infty$ เ $\dot{0} \dot{\infty} \dot{0} \dot{\infty} \dot{\infty} \dot{0} \dot{\circ}$ 군

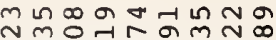

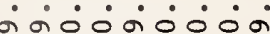
U GR ORR m mm m mm

- $N m+40 \infty$

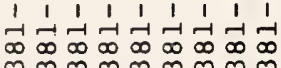
웅ㅇㅇㅇㅇㅇㅇㅇ $\begin{array}{llllllllll}1 & 1 & 1 & 1 & 1 & 1 & 1 & 1 & 1\end{array}$

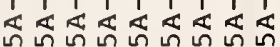
$\begin{array}{lllllllll}1 & 1 & 1 & 1 & 1 & 1 & 1 & 1 & 1\end{array}$

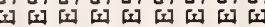

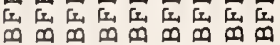




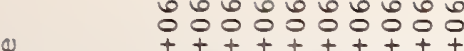

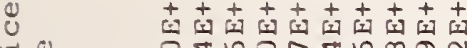

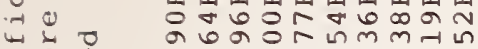

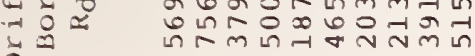
$\dot{\sim}-\dot{-} \dot{\sim}-\dot{\sim}-\dot{v}-\dot{v}$

에 $\infty \pi 0 m m \infty$

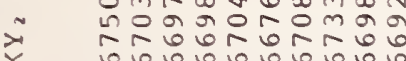
$\ddot{x}$

융ㄴํ은 $+0-1-00 \times-1$

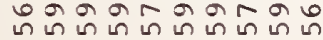

भกต $\dot{m} \dot{\sim} \dot{\circ} \dot{i} \dot{\sim} \dot{0} \dot{0}$

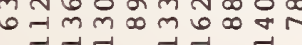

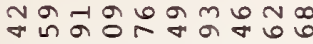
मे जि -

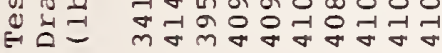

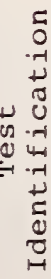

-1 $\begin{array}{llllllllll}-1 & -1 & -1 & -1 & -1 & -1 & -1 & -1 & -1 \\ \infty & \infty & \infty & \infty & \infty & \infty & \infty & \infty & \infty & \infty\end{array}$

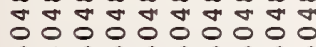
○ 006006060060

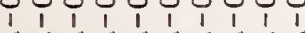

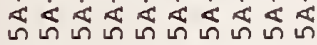
$\begin{array}{llllllllll}1 & 1 & 1 & 1 & 1 & 1 & 1 & 1 & 1 & 1 \\ 1 & 10 & & 1\end{array}$ जीञ

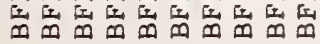

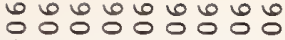

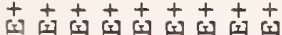
mo

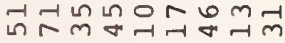

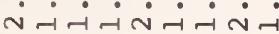

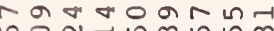
$60 \mathrm{~N}$ 는 ชิซี ல் $0000 \dot{0}$

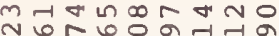
눙ำ

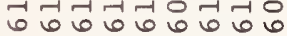
○ं0ல்000

부뉴ำ ન $\infty$ बून

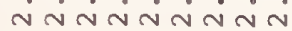

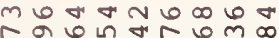
กิ์

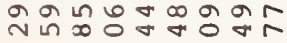
$\dot{ } \dot{m} \dot{\sim} \dot{\sim} \dot{\sim} \dot{\sim} \dot{\sim} \dot{\sim}$

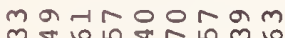

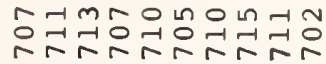
ใ 606060 ข ○ं $0 \dot{0} 000 \dot{0}$

nmantoram N. T.

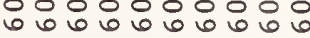
$\therefore \circ 00000000$

ฟ

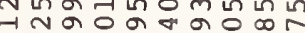
○๐ m $\sim m \sim N \sim m \sim N$

ㄱำ

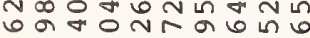
ก 0 ล $\mathrm{N}$ ก

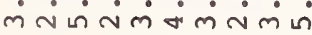

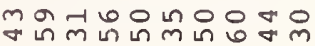

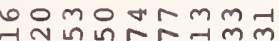

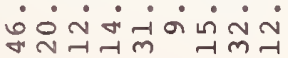

เกษ $\infty \neq \infty r m b l \neq a$

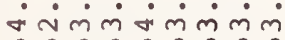
०6 6060606

ตำ ब대 $\dot{-1} \dot{0} \dot{0} \dot{0} \dot{0}$

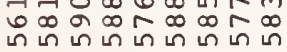

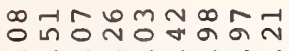

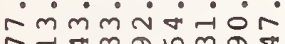
न

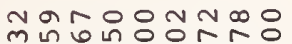

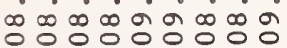
의

-

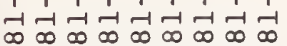

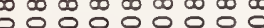
กับก ก ก ก 000000000 ૭૭

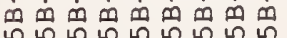
जी

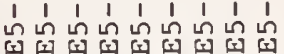

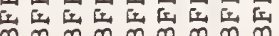

ำ

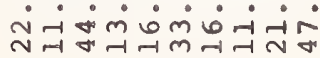

กตก แง ง ต $\therefore \infty \infty \infty \dot{\sim} \infty \dot{\sim} \infty$

ข้กิักิ์ $\dot{2} \dot{0} \dot{0} \dot{0} \dot{0} \dot{0}$ กิ

तronomana ㄴ.? Nํำ

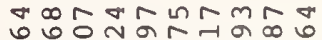
$\dot{\sigma} \dot{\sigma} \dot{\infty} \dot{\sigma} \dot{\sigma} \dot{\sigma} \dot{\sigma}$

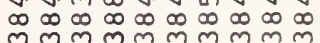

$\rightarrow-1-1-1-1-1-1-1$ $\infty \infty \infty \infty \infty \infty \infty \infty \infty$ nกNNNNnNnN

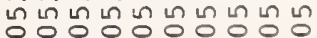
1 0000000000

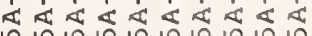
ที่ ○ 00000100

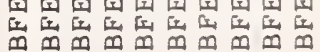

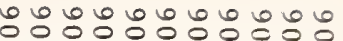

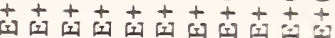

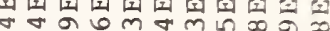
我 งัง $\dot{-} \dot{\sim} \dot{\sim} \dot{\sim} \dot{\sim} \dot{-} \dot{-} \dot{-}$

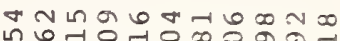

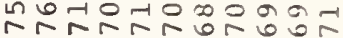

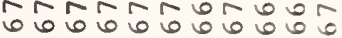
000000000

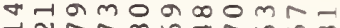

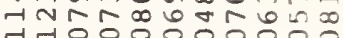
-1 ல0ல0ல0்000

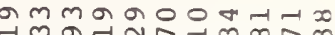

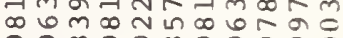
न ब ก ก ก ก N N

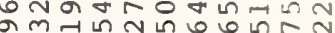

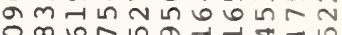
웃ำ ํ்ம்ம் ம்

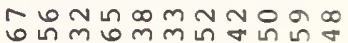

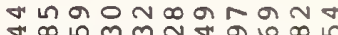
$\dot{-i n} \dot{-1} \dot{m} \dot{0} \dot{-} \dot{-} \dot{0}$

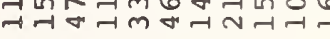

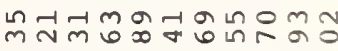
$\infty \infty \sigma \infty \infty \infty \infty \infty \infty$

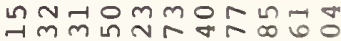
-mó்

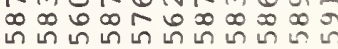

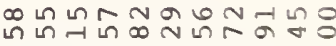

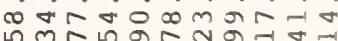

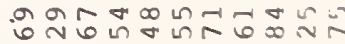
$\therefore \dot{m} \dot{m} \dot{\sigma} \dot{0} 0$

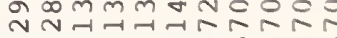

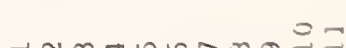
$\begin{array}{llllllllll}1 & 1 & 1 & 1 & 1 & 1 & 1 & 1 & 1 & 1\end{array}$

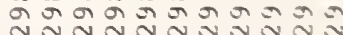

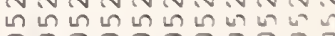

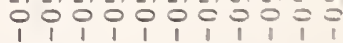

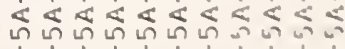

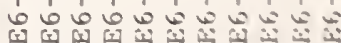
車

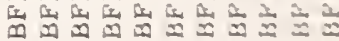



ल

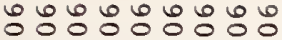

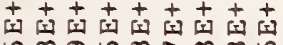
oma

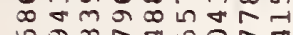

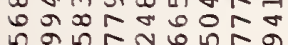
- $-\dot{i} \dot{-i} \dot{-i}-\dot{i}$

Oก

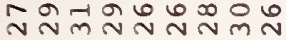
nNinknin

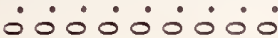

- amamorn

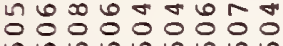
666606606

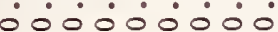

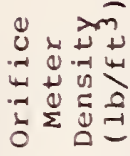

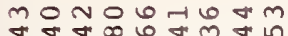
西

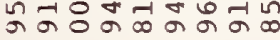
vं $\dot{\sim} \dot{\sim} \dot{\sim} \dot{\sim}$

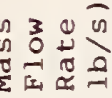

ㄱำ ํำ ボNNํำ

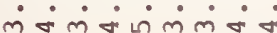

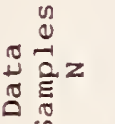

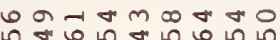

- in $^{m}$

4 of 0 I

फ

0 工 2.

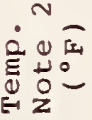

กீ •?.? ?. ?

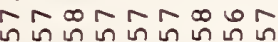

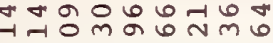
$\rightarrow \infty-\dot{\sim} \dot{\sim} \dot{0} \div$

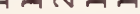

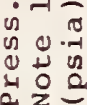

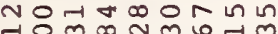
- $\cdot \dot{0} \cdot \dot{0} \cdot$

प1

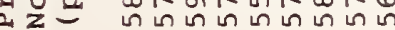

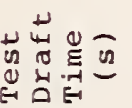

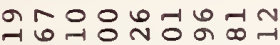

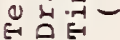
4
4
0

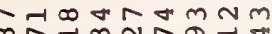

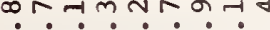
ம்m户் $\dot{m} \dot{\sim}-1$

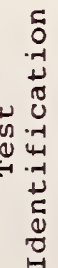

TNm ง $\infty \infty \infty \infty \infty \infty^{\infty} \infty \infty^{\infty} \infty \infty^{\infty} \infty$

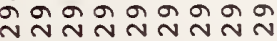

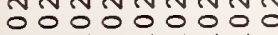

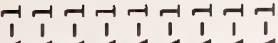

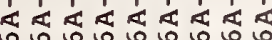
$\begin{array}{llllllll}1 & 1 & 0 & 0 & 0 & 0 & 0 & 1\end{array}$

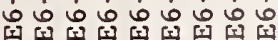

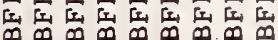

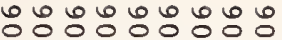
$+++++t+t+$ or जी ช ขㅇํำ . $-\dot{N-1}-\dot{N-1}-\dot{-}$

nothan

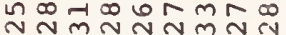
nnminnm $\dot{0} 0 \dot{0} 000000$

$m \omega-\pi m a m o$

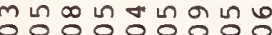
๑ 60060600 0000000.0

$\infty m \square \infty \forall N-1 m$ กம

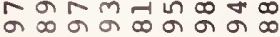
งผ่งกัง

an L NOサ

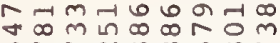
or $\dot{m} \dot{\sigma} \dot{\dot{\sigma}} \dot{\dot{m}} \dot{m} \dot{\sigma} \dot{\sigma}$

유ำผำ

เํำ $\dot{0} \dot{0} \div \dot{-} \dot{-} \dot{\circ} \dot{0}$

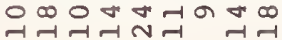

읨ำ *mเ? $\infty N \infty \infty N \infty N$ o แก เก เก เก เก แก เก เก เก

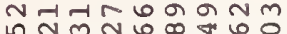
$\dot{0} \dot{0} \dot{0} \dot{-1} \dot{0} \dot{0}$

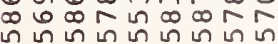

กำ ㅇํำ ड

븡ㅇ윻ㅇㅇㅛ $\dot{0} \dot{\dot{m}} \dot{\sigma} \dot{\dot{m} \dot{m}}$

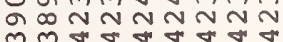

-1 $⿻$ m ง.n.

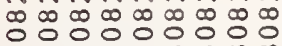

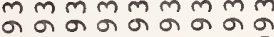
O5: 00000 1 11111111

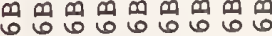
$\begin{array}{llllllllllll}1 & 1 & 1 & 1 & 1 & 1 & 1 & 1 & 1\end{array}$

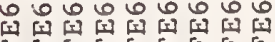

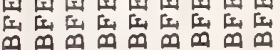

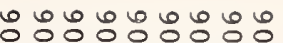

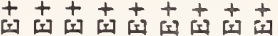

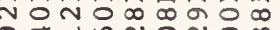
$\sigma *-1$ in $N$ Nom ฟ $\dot{-1} \dot{-i} \dot{\sim} \dot{-i} \dot{-i}$

$\infty$ \#-1

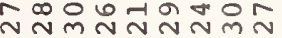
nnm NNNNMN Oல்0ல்ட0்

- Imo In No In in 웅요용요 ○ं $\dot{0} 0 \dot{0} 0 \dot{0}$

OImmNNING m-17-10 $\infty N$ in

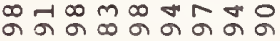

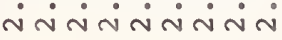

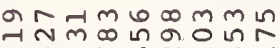
- $N$ a

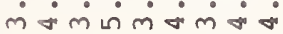

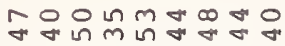

m $0 \infty-\dot{0} \dot{0} \dot{\sim} \dot{\sim}$ -1

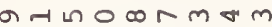
$m \omega$ เก แก $m-1$ แก

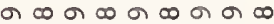
แก เก เก เก เก เก เก เก

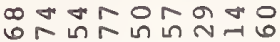
$\dot{0} \dot{0} \dot{0} \dot{0} \dot{0} \dot{\sim}$ mm $m 06-1$

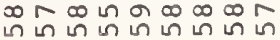

a o R ONG

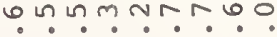
-ம் $\dot{0} \dot{\sigma} \dot{m} \dot{\sigma}$ 0 $\infty$ O

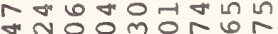
- OOم்́

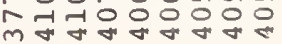

- $1 m+4 \infty \pi \infty$

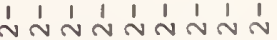

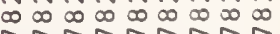
000000000 걱더더더다

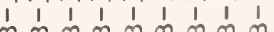

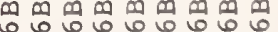
$\begin{array}{lllllllll} & 1 & 1 & 1 & 1 & 1 & 1 & 1 & 1\end{array}$

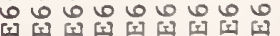

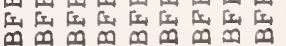


Table 9(a). Test Meter Identification and Physical Measurements Nominal 6 Inch AGA/API Orifice Meter (British Units).

$$
\text { Test }
$$

Identification

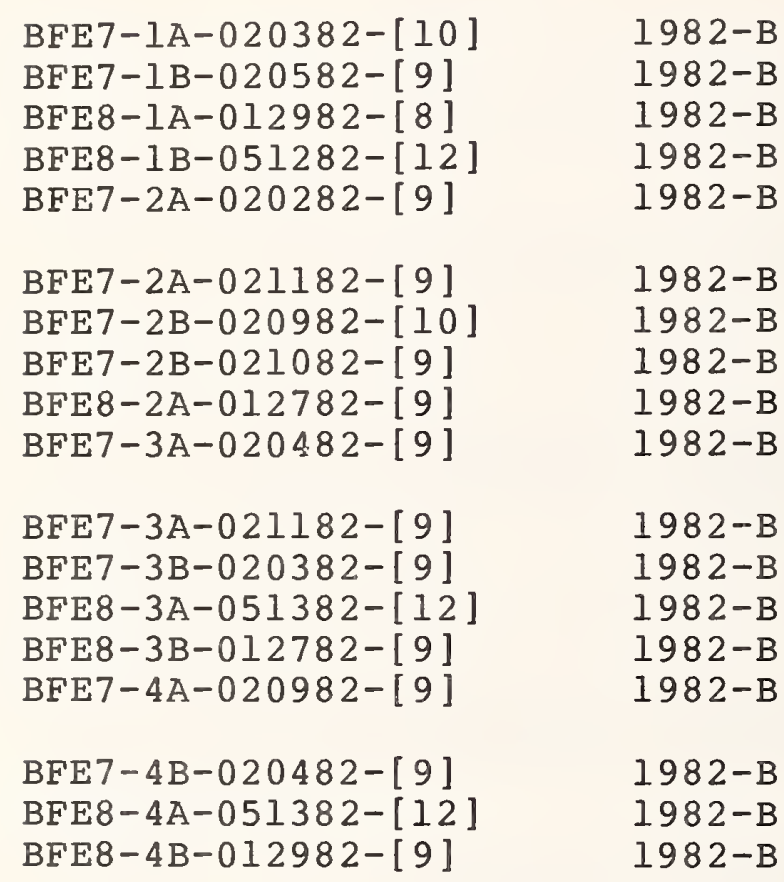

Test
iguration

Run
Diameter
(inches)

Orifice

Diameter

(inches)

$\begin{array}{lll}6.0698 & 1.2490 & 0.2058 \\ 6.0698 & 1.2491 & 0.2058 \\ 6.0693 & 1.2490 & 0.2058 \\ 6.0693 & 1.2491 & 0.2058 \\ 6.0698 & 2.2499 & 0.3707\end{array}$

6.0698

2. 2499

0.3707

6.0698

2. 2500

0.3707

6.0698

2. 2500

0.3707

6.0693

2. 2499

0.3707

6.0698

3.0003

0.4943

6.0698

6.0698

3.0003

0.4943

6.0693

3. 0004

0.4943

6.0693

3.0003

0.4943

6.0698

3.0004

0.4944

3.4994

0.5765

6.0698

6.0693

3.4993

6.0693
3. 4994

3.4993
0.5765

0.5766

0.5766

Note: Test Identification bracket gives number of tests run with this geometry. A total of 172 tests were run on the nominal 6 inch run size. 


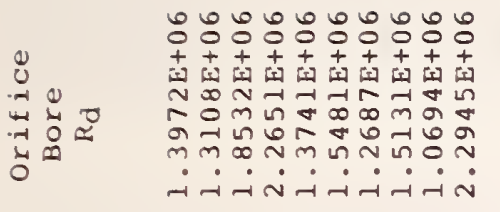

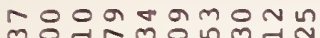
× 0000000000

ก้กนMตกㄴำ

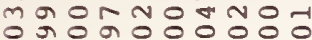
$\stackrel{-1}{\pi}$
ல $\dot{0} \dot{0} \dot{0} \dot{0} \dot{0} \dot{0}$

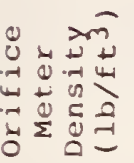

ก ప $\dot{m} \dot{x} \dot{m} \dot{m} \dot{m} \dot{\sim}$

is 300

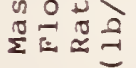

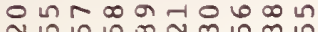

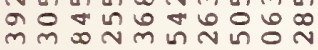

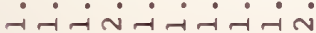

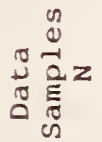

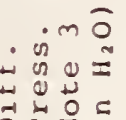

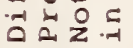

กํำ mேन

D-1 กแกน์ ㅇํ의

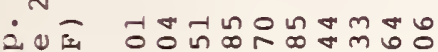
ह닝 ${ }^{2}-$ réntánom

(i)

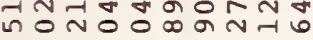

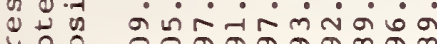
ப之

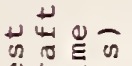

ํํㅇㅇำ

E \&

คำก

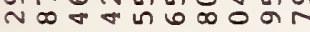
iñ

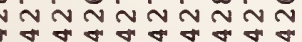

- 1 m

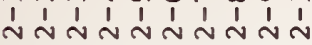

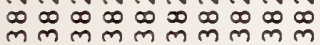

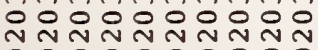
O O O O O 0000

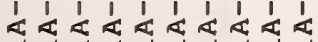

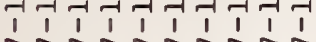

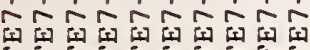

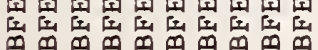

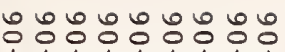

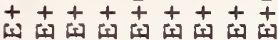

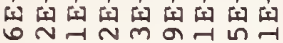
ำสำกำ ชง

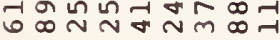

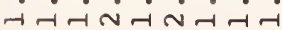

mmMn $m$ n พูกตรส

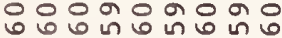
ல் 000000

$\infty \infty$ 어요묘

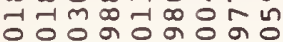
606 in 6 in 6 in ○ं $0 \dot{0} \dot{0} \dot{0} \dot{0}$

เ

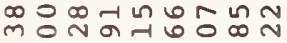

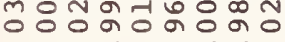
$\dot{m} \dot{m} \dot{N} \dot{m} \dot{m}$

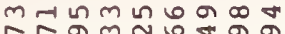
न 6 in in 7 \%

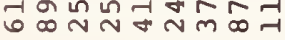

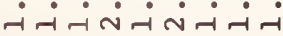

엄

มกตก 约然 の

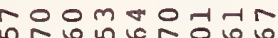
.

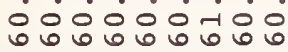

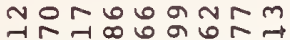
-i் $\dot{\theta} \dot{-1} \dot{0} \dot{0} \dot{0}$ ํำ

พ ไกต

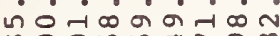

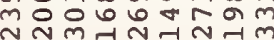

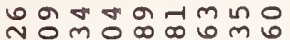
$\dot{000} \dot{0} \dot{\sim} \sim$ m户n

- $N$ m n ง่ง

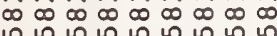

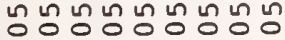

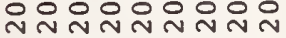
o 00000000

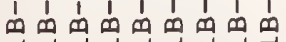
-1 rarrar

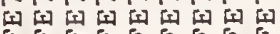

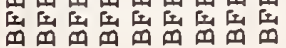

ீீீீ: t十南南南+十+ $0 n-10$ जी $\infty$

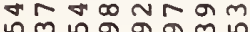

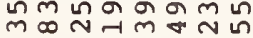

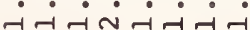

으네에에 ०००न 600 ำ ○ं0் 0000

แn

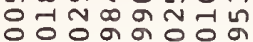
○ㅇํำ in 60 in ○ 0 0 0000

m요의 0 의 훙ㅎㅀ ०ू नू नू नू नू न mंพ

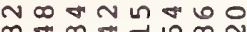

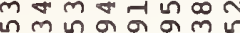

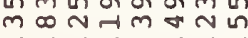
A-

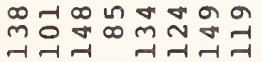

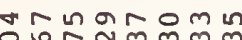
o

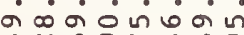

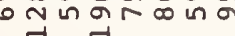

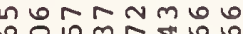
•. in 00000000

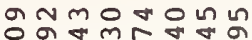
$\dot{0} \dot{0} \dot{\circ} \dot{\sim} \dot{0}$

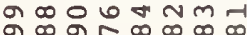
กำ

$\infty \infty \forall \backsim N=$

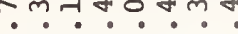
OURNONN

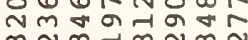

ดกษ $\dot{m} \dot{m} \dot{m} \dot{m} \dot{m} \dot{m}$ $m m m m m m m m$

INm

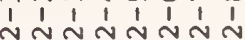
$\infty \infty \infty \infty \infty \infty \infty \infty$ \% \% กำกำ กิ

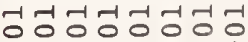

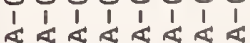

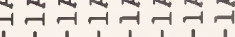
$\begin{array}{llllllll}1 & 1 & 1 & 1 & 1 & 1 & 1 & 1 \\ 0 & 0 & 0 & 0 & 0 & 0 & 0 & 0\end{array}$

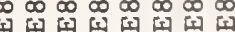
政

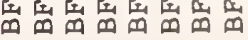

○:

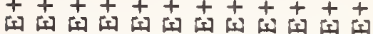
กั่ㅇㅇㅇㅇㅢ तनाराष्म 슴ำ

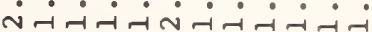

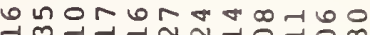

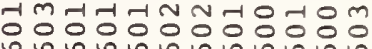
•. 000000000000

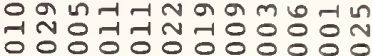
6 60606060606 000000000000

ดกน

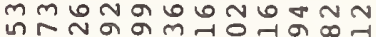

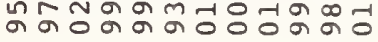
Nं山் $\dot{N} \dot{\sim m} \dot{m} \dot{N} \dot{m}$

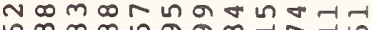

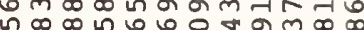
ำ

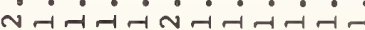

ฌั

กตำ omm o a m ட் $\rightarrow-1$

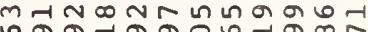
$\cdot \bullet \cdot \bullet \cdot \cdot \cdot \dot{0} \cdot$ กู

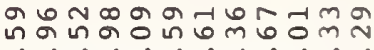

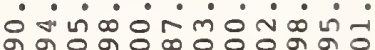

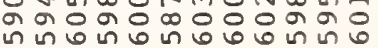

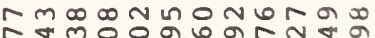
?.?.?. ดั न

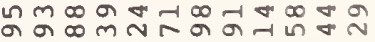

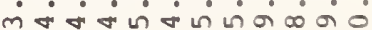

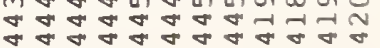

Ham ง

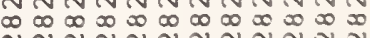
N N N N N N N N N

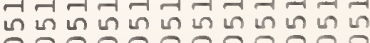
○0 000000000

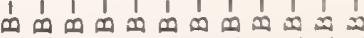

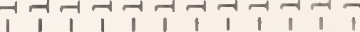

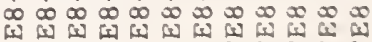

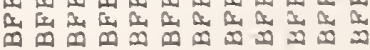




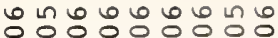
U) $\quad+++++++++$ - 1 क แ

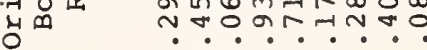
$\dot{-1} \dot{-1} \dot{\sim} \dot{\sim} \dot{-} \dot{-}$

N $\quad$ का 구 نं0்0ं0.0



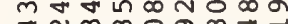

त

U 000000000

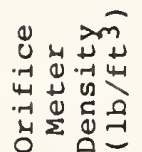

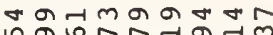
กूळ

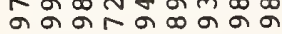

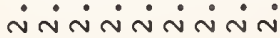

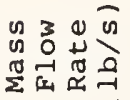

Lก N ㄷㅇㅇㅇำ

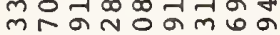
$\dot{\sim} \dot{-} \dot{\sim} \dot{m} \dot{\sim} \dot{\sim} \dot{-}$

离

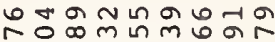

- 20

- in in

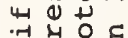

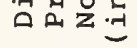

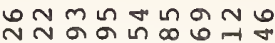

$\dot{0} \dot{0} \dot{0} \dot{0} \dot{0} \dot{0}$

$\rightarrow \neg 0 \mathrm{~m} \in \rightarrow-1$

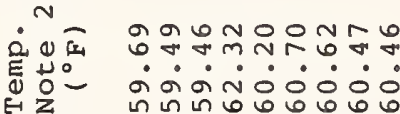

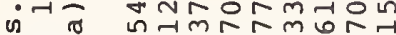

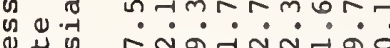
म 出 2

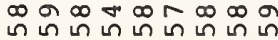

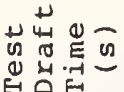

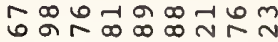

- $\dot{0} \dot{0} \dot{0} \dot{0} \dot{0}$ andr.

ฟึั 㟧 सम

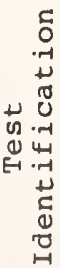

14

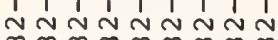

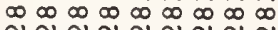

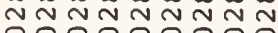
응ㅇㅇㅇㅇㅇㅇㅇㅇ nN nN N N N N 0 O 0000000

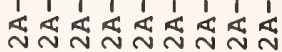
$\begin{array}{lllllllll}1 & 1 & 1 & 1 & 1 & 1 & 1 & 1 & 1\end{array}$

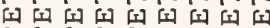

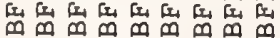

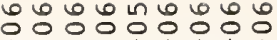

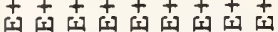

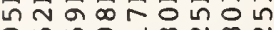
ब 1 ⿰纟勺-

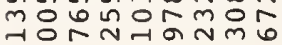
-imini $\dot{i} \dot{i}$

om mo

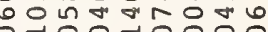
ำ 60676060 ○ं $0 \dot{0} 0 \dot{0} 0$.

$\infty$ n $0 m \omega n \nleftarrow m$

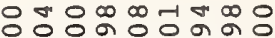
060 n 60 แn ○ 0 000000

ํㅜำกำกำ ชำ míñ

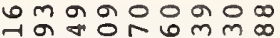

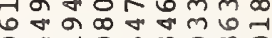
o frolomomo ஸ்ம் $\dot{\sim} \dot{-1} \dot{\sim} \dot{\sim}$

$\backsim N \backsim m \backsim m m \nabla \infty$ ח

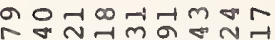
$\therefore-\dot{-100} \dot{0} \dot{0}$ ન

โ ‥? $\dot{m} \dot{m} \dot{m} \dot{\sigma} \dot{\sim}$ ด

ํㅜㅇㅠ

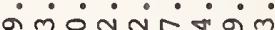

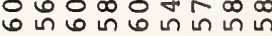

느의 $m \infty \circ \infty 9$ $\infty r \cdot v \pi-7 m a n$ N ormonn

그ำ óñrínir

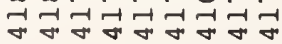

INm ง $\infty \infty \infty \infty \infty \infty \infty \infty$

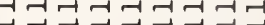
nNNNNNNNN O O O O O O O O $\begin{array}{lllllllllllllllll}1 & 1 & 1 & 1 & 1 & 1 & 1 & 1 & 1\end{array}$ 芯芯芯芯芯芯芯 $\begin{array}{llllllll}1 & 1 & 1 & 1 & 1 & 1 & 1 & 1\end{array}$ 되되되 되되되 되되 되

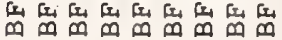

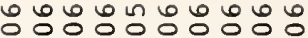

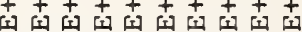
ब约 $\sigma$ त $60 \mathrm{~m}$

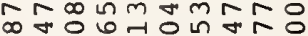

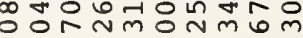

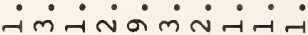

mก片

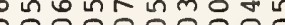

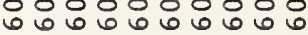
○ं 0000000

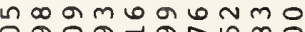

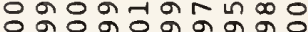

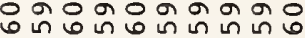
○ं 0 0 000000

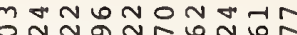

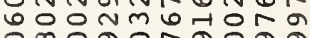
mกMNMNMN

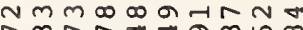

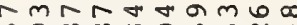
๙ iம $\dot{0} \dot{\sigma} \dot{\sim} \dot{\sim} \dot{\sim} \dot{m} \dot{\sim}$

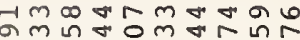

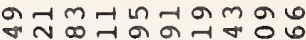

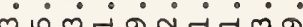
$m$ 데

$\infty$ ก

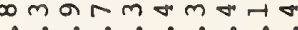

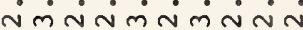
ט

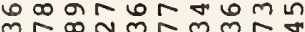
- $\dot{0} \dot{0} \dot{0} \dot{0}$ oñ 은

$\forall \infty$ ในก mंம்án $\dot{\sim} \infty$

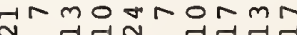

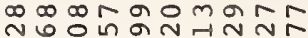

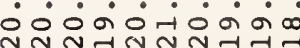

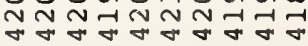

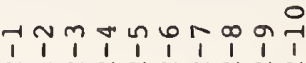

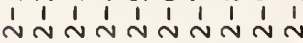
$\infty \infty \infty \infty \infty \infty \infty \infty \infty \infty$ न न न न न न न न न

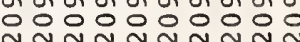
OOOOOOOOOO

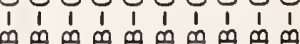

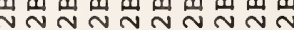
$\begin{array}{llllllllll}1 & 1 & 1 & 1 & 1 & 1 & 1 & 1 & 1 & 1\end{array}$

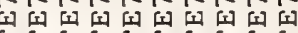

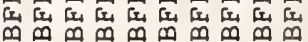

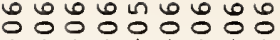

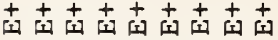

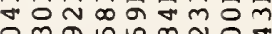
Oma n 0 No

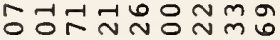

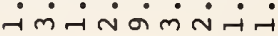

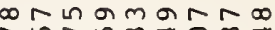
듀ำ

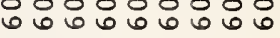
○ं $0 \dot{0} \dot{0} \dot{0} \dot{0}$

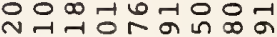
००००ส 6000 ต ○ं0ல்000

กักษ์

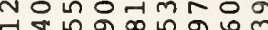
ชิ mกं

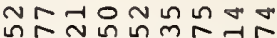

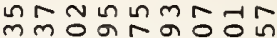
ตำ의

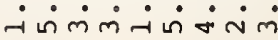

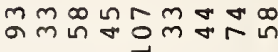

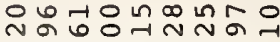
mं $\dot{0} \dot{0} \dot{0} \dot{0} \dot{0}$ H.

טNm $m \sigma \infty-10$

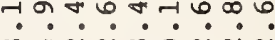
maN MONN

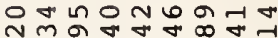
$\dot{0} \dot{0} \dot{\sigma} \dot{\sigma} \dot{\sigma} \dot{0} \dot{\circ}$ の

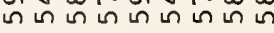

ดก Oxm $6 \dot{\sigma} \dot{0} \dot{0} \dot{0}$

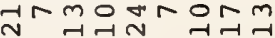

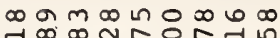
- $\dot{0} \dot{0} \dot{0} \dot{0}$

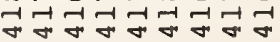

HNm

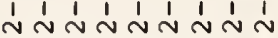
$\infty \infty^{\infty} \infty \infty^{\infty} \infty \infty^{\infty} \infty \infty^{\infty} \infty$ 000000

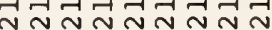
00 0000000 $\begin{array}{lllllllllllllllll}1 & 1 & 1 & 1 & 1 & 1 & 1 & 1 & 1\end{array}$

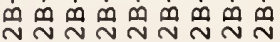

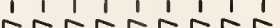

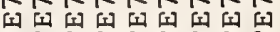

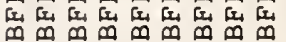


(1)

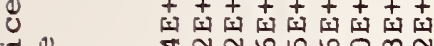

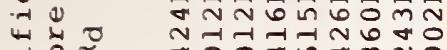

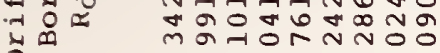

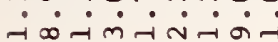

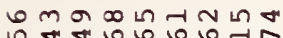
خ ○ं $0 \dot{0} 0 \dot{0} 00$

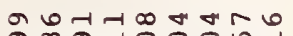

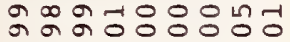

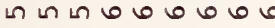
000000000

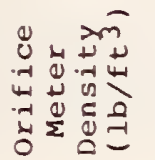

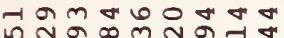
$\infty$ ॠ

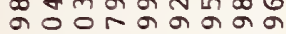
نंलिं $\dot{\sim} \dot{\sim} \dot{\sim}$

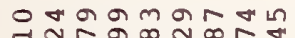
สำ

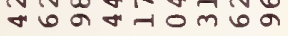

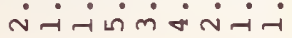
幽

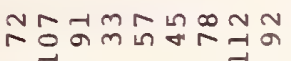
- $m \widehat{0}$ i is o w

ontoamonin

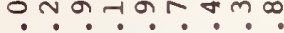
तोंmm $\sim$

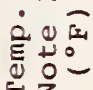
NNmmaG-INA

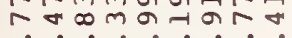
бَ ㄱำ ด. NMNN

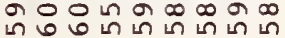

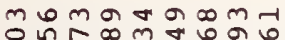

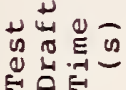
- $\dot{0} \dot{0} \dot{0} \dot{0} \dot{0}$

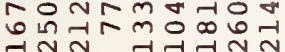

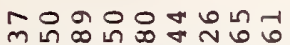
4
0
0
0

TNm

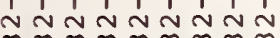
$\infty \infty \infty \infty \infty \infty \infty \infty \infty$ ก กัก กับก

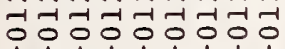

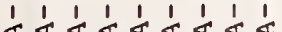
芯芯芯芯芯芯 $\begin{array}{llll}1 & 1 & 1 & 1\end{array}$

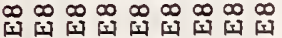

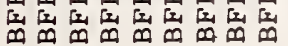

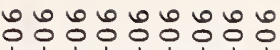

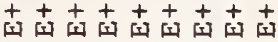

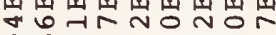
ogri no non

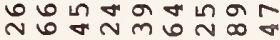
ก.

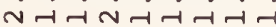

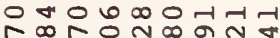

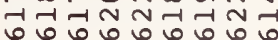
○ं ல் 0000

mกm⿻ कmm

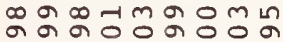

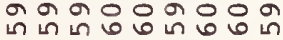
000000000

madnatan

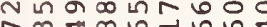

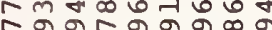
iจ

6ำ

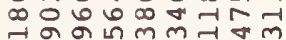
अ बे वे

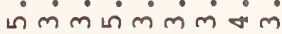

๓กษ

-

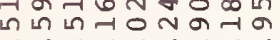

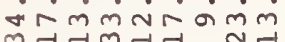

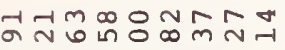
ด เ

$-\infty \infty 6 \omega 0 N-m$ न 0 ด

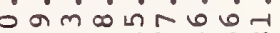

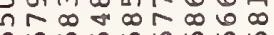

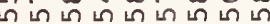

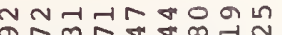
- $\dot{0} \dot{\sim} \dot{0} \dot{0}$ - 0 ㄴำ $N$ ก

ต๑ตm कr. m-i $\min \sim \mathbf{N}$

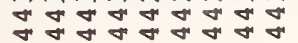

4nm

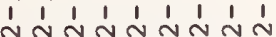
$\infty \infty \infty \infty \infty \infty \infty \infty \infty \infty$

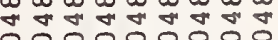

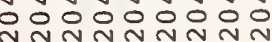
O O O O O O O O

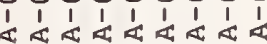
लिलmmलm

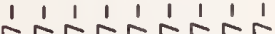

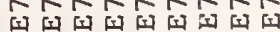

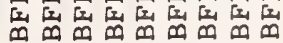

○ $+t+t+t+t+$

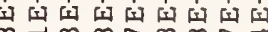

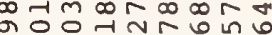
ก어요 ฟ $\dot{-i} \dot{-1} \dot{\sim} \dot{-i}$

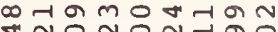
กำ $\sim$ ก

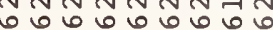
○ं0ல0ல000

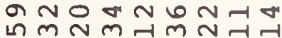
เกตกำกำ

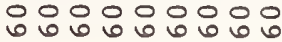
000000000

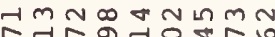

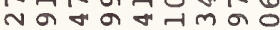

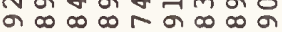
vं山 $\dot{\sim} \dot{\sim} \sim$

ㅇㅇㅇำ Noom $0 \infty$ เก กี $\mathrm{N}$ ก ᄂกำ

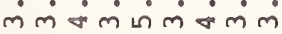

ஸึเกำํำ กพก

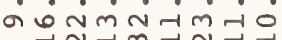

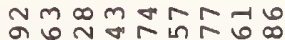
$\dot{0} \dot{0} \dot{-1} \dot{-1} \dot{-1}-\dot{0}$

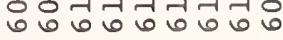

6 เก กน and inuraror

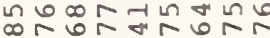

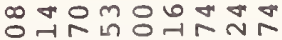

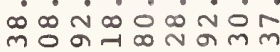
ना一

n m a a a m

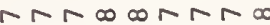

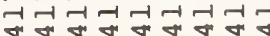

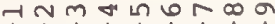
$\begin{array}{lllllllll}1 & 1 & 1 & 1 & 1 & 1 & 1 & 1 & 1\end{array}$ $\infty \underset{\infty}{\infty} \infty \infty \infty \infty \infty$

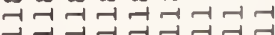
กัก กัก กัก 000000000

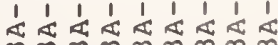
i $1,1,1,1$

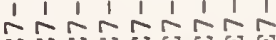

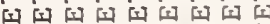

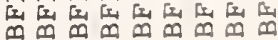
ดั

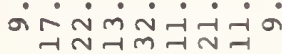

응ำตำำ íáióá

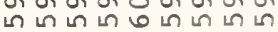

ดกำกำ 00 0 in

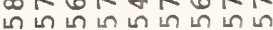

ำ $m$ ก

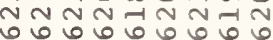
000000000

4am nat

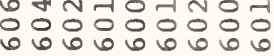
000000000

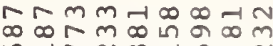

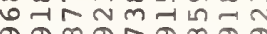
ก N N N N

เก ตी $\dot{m} \dot{m} \dot{\dot{m}} \dot{\dot{m}} \dot{\mathrm{v}} \dot{\mathrm{N}}$

సु $m$ ต

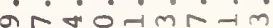
mó ते $\infty$

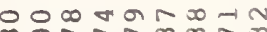
0

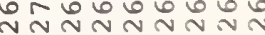

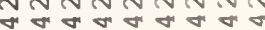

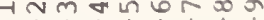
N N N N N N N

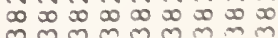
잉ㅇㅇㅇㅇㅇ NON N N N N 1 1 1 1 1

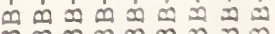
। 1 1 । 1 । ।

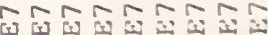

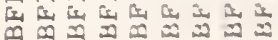

ㅇำ 


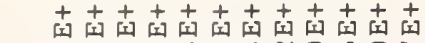
- $\begin{array}{ll}-1 \\ -1\end{array}$

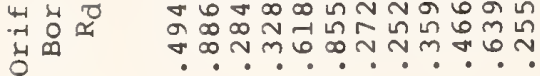
- $-N-\pi-N \rightarrow-n-1$

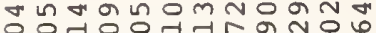

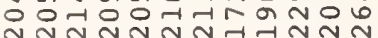

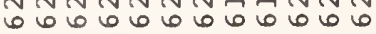
000000000000

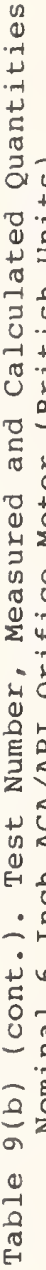

on خิ

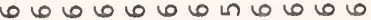
○ं0 0000000

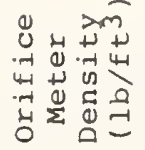

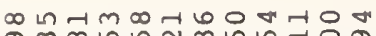
న $\infty$ w नळ ก

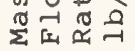

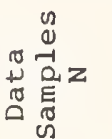

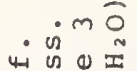
4 व 4 兽㞻的

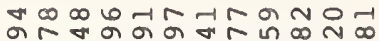
ம் is $\begin{array}{lll}4 & 1 & 0 \\ 4 & 0 & 0 \\ 0 & 2\end{array}$

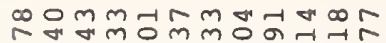
ம

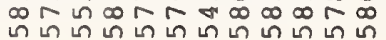

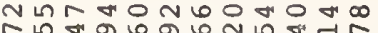
o

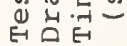

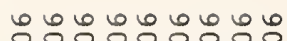

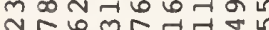
ส-1ำกำกำกำ

in 3 is is

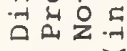
雚蒈学

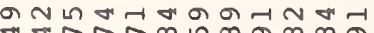
○ं0ं் $\dot{0} \dot{0} \dot{0} \dot{0} \dot{0}$ N $N \rightarrow$ 네 $\mathrm{N}$ เก

オNm-

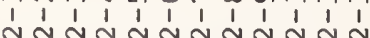

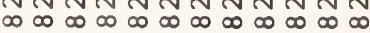
mmmmmm

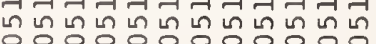
l 1 l 1

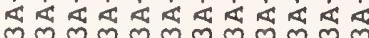

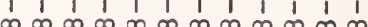

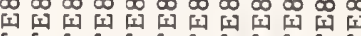

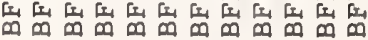

แn $\infty \infty \cdots N$ \% $++t+t+t+t$ $m \infty-1060$

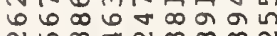
ง 0 \% - $\dot{-1} \dot{\sim} \dot{\sim} \dot{-1}$

Nmm $n \infty \pi n$

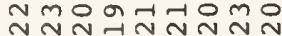
ช 60606060 000000000

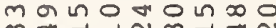

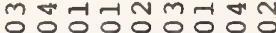
6606406106 ○ंல்ல0ல0

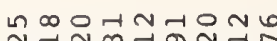
N

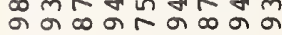
กNกNกNกN

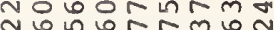
N้눈 लㅇํㅇ जี जึ $\dot{m} \dot{\sigma} \dot{\dot{m}} \dot{m} \dot{\sigma} \dot{m}$

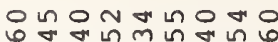

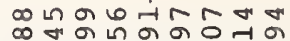
वंतिलंखिं भN . 0.0. 0்َ

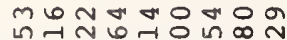
$\dot{0}-\dot{\sigma} \dot{m} \dot{\sigma} \dot{\infty} \dot{-}-\dot{-}$

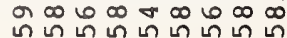

요인 o 6 R

ㅇํㅇ 웜

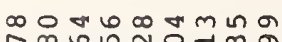
ก. $\dot{\sim} \dot{\sigma} \dot{\sigma} \dot{\sim} \dot{\sigma}$ $\mathcal{N} N \underset{N}{N} \mathcal{N} N$

-Nm

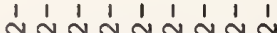
$\infty \infty \infty \infty \infty \infty \infty \infty \infty$ ำกำกำกับ 겅더엉어엉어엉어 1 l 1 品品品品品 १ल लmलm

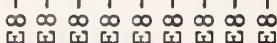

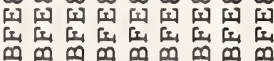

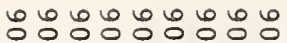
南南南南南南南血南 ㅅำ

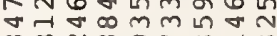
$\infty \infty \pi \infty$ or $m-1 m$ robr $\infty$ in in in

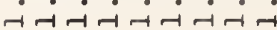

๒ํㅇㅇำ mㅇํ요 m

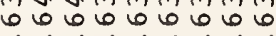
○ं0 00000

m⿻의 0 웅

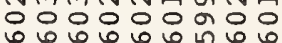
○ंல் $0 \dot{0} 0$

พั $\infty \sim \infty \infty$ ก Nํㅇำ

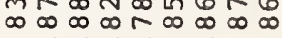

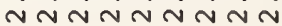

om $m m \in$ का

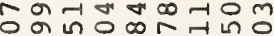
० 0 แก 0 N

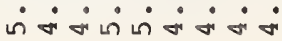

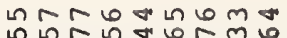
$\therefore \dot{\sim} \dot{\sim} \dot{\sim} \dot{0} 0$

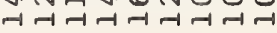

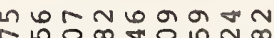

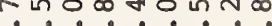
ن்

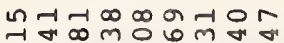
$\dot{*}-\dot{-1}-\dot{\sigma} \dot{-1} \dot{0}$

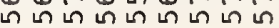

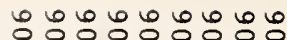

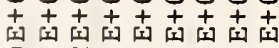
NNㄱㅇㅇㅇㅢ

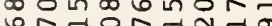
0 in เू $-1-1-i-1+1$

-กต 웅

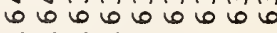
000000000

-manlan

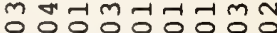
6060606060 ○ं0 00000

mag

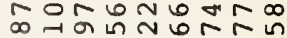

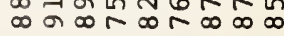
กงกนกนกนก

$\infty \circ \omega \omega L \infty N N$

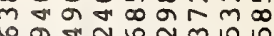
ตั $\dot{\sigma} \dot{\sigma} \dot{0} \dot{0} \dot{0} \dot{0} \dot{0}$

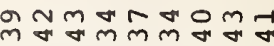

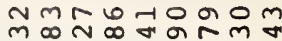

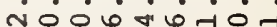
걷ㄷㄷㄷ

เ $\dashv$ ○

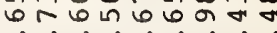

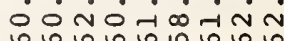

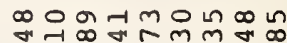
- $\dot{0} \dot{1} \dot{0} \dot{0} \dot{0} \dot{0}$

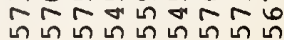

অnN $\cdot \bullet \cdot \cdot \cdot \cdot \dot{0}$

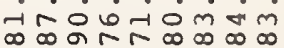

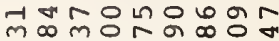
-i $\dot{0} \dot{0} \dot{0} \dot{0} \dot{0}$

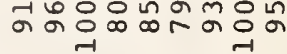

กพ⿻mก⿻上丨 m. مं0- $\dot{0} \dot{0} \dot{0} 0$

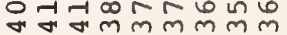

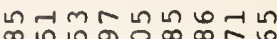

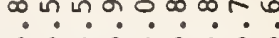

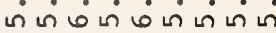

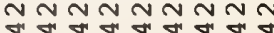

-1

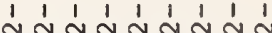
$\infty \infty \infty \infty \infty \infty \infty \infty \infty \infty$ बन न न न न न क

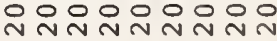
O O O O O O O O

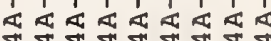
$\begin{array}{lllllll}1 & 1 & 1 & 1 & 1 & 1\end{array}$

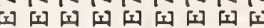

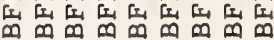

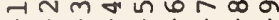

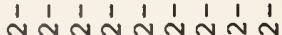
$\infty \infty \infty \infty \infty \infty \infty \infty \infty$

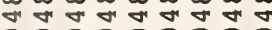

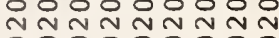
이잉ㅇㅇㅇㅜ m m m m m m

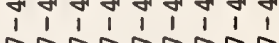

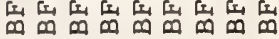

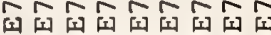




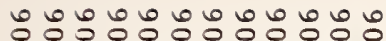

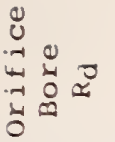

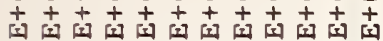

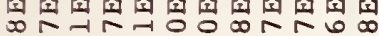

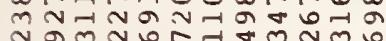
กิก $\dot{\sim} \dot{\sim} \dot{-\dot{\sim}} \dot{-\dot{\sim}} \dot{-\dot{\sim}} \dot{-}$

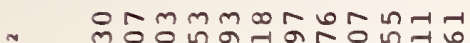

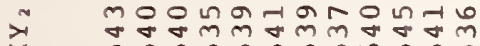
6 6 6 ல0000000000

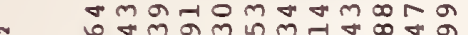

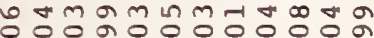
606 in 6060606 in ○ं் $\dot{0} \dot{0} \dot{0} \dot{0} \dot{0}$

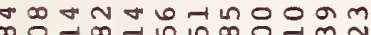
0.? aOnammnNano

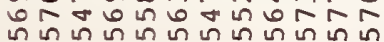

๓ન - $\dot{1} \dot{0} \dot{\sim} \sim \dot{0} \dot{0} \dot{0}$

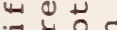

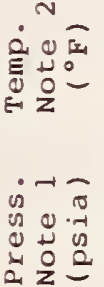

น $\therefore \dot{\sim} \dot{\sim} \dot{\sim} \dot{m} \dot{\sim} \dot{\sim} \dot{0}$ ซึ

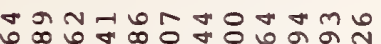

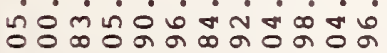

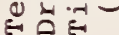

ט

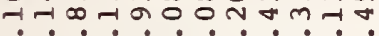
N-1 $-100 \dot{-1}-\dot{1}-\dot{1}$

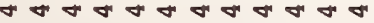

-1

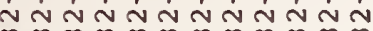

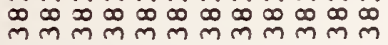

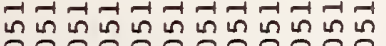
i $\begin{array}{lllllllllll}1 & 0 & 0 & 0 & 0 & 0 & 0 & 0 & 0 & 0 \\ 1 & 1 & 1\end{array}$

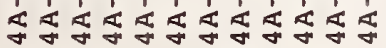
$\begin{array}{llllllllllllll}1 & 1 & 1 & 1 & 1 & 1 & 1 & 1 & 1 & 1 & 1\end{array}$ $\infty$

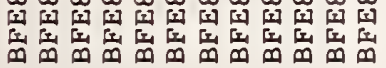

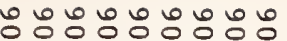

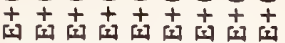
No 0 mm

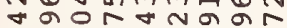

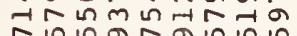
i $\dot{1}$

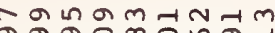

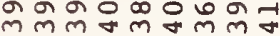
० 0606000 000000000

mNㅐ요용 mm

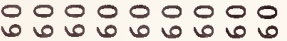

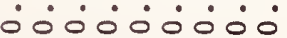

응ํํㅇำ ตํํำ

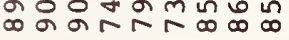
ن $\sim \dot{\sim} \sim \dot{\sim}$

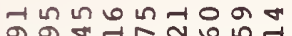
क-

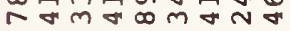
$\dot{\sigma} \dot{\sigma} \dot{\sigma} \dot{\sigma} \dot{\sigma} \dot{\sigma} \dot{\sigma}$

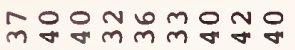

임ㅇㅁ음 $-\infty$ . . . . ?

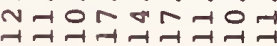

ตำm ○ं $\dot{0} \dot{-1} \dot{0} \dot{-1} \dot{0}$ 0 6060606

ฟำ ?. ? ? ?

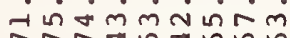

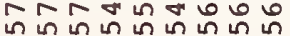

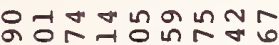
- vंminmin dáñóna

윢ํㅇㅇㅠ - $\cdot 0 \cdot \dot{0}$ 0 $00 \% \forall m$ 영여의

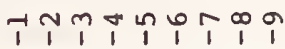
NNNNNNNN

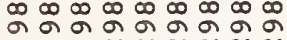
ก ก ก ก ก ก ก

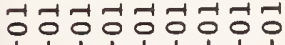
$\begin{array}{llllllll}1 & 1 & 1 & 1 & 1 & 1 & 1 & 1\end{array}$

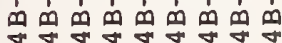
1
1

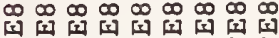

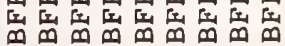


1. Dean, J. W., J. A. Brennan, D.B. Mann and C. H. Kneebone, Cryogenic flow research facility provisional accuracy statement, Nat. Bur. Stand. (U.S.) Tech. Note 606 , (1971).

2. Mann, D. B., and.J.A. Brennan, On a new method of gas flow measurment using cryogenic techniques, Nat. Bur. Stand. (U.S.) Special Pub. 484, vol. 2, pp 881-93, (1977).

3. A magnetic tape containing all measured published data in SI units is available at cost of reproduction from Chemical Engineering Science Division, Chemical Engineering Center, National Bureau of Standards, Boulder, C0 80303.

4. Mann, D. B., J. A. Brennan, C. F. Sindt, J.F. LaBrecque and C. H. Kneebone, Gas orifice meter performance with new flow reference system, Proceedings of 1983 Int. Gas Research Conf., London, UK, (June 12-16, 1983).

5. Powel1, R. L., W. J. Ha 11, C. H. Hyink and L. L. Sparks, Thermocouple reference tables based on IPTS 68 , Nat. Bur. Stand. (U.S.) Monograph 125 (1974).

6. American National Standards Institute, ANSI/API 2530, Orifice metering of natural gas, American Gas Association, 1515 Wilson Boulevard, Arlington, VA 22209, (1978).

7. Younglove, B. A., Thermophysical properties of fluids.1. argon, ethylene, parahydrogen, nitrogen, nitrogen trifluoride, and oxygen, J. Physical and Chemical Reference Data, 11, Supplement No. 1, (1982).

8. American Society of Mechanical Engineers, Fluid meters, their theory and application, 6th Ed., American Society of Mechanical Engineers, 345 East 47 th Street, NY,NY, pp 181-82, (1971).

9. Sprenkle, R. E. a and N. S. Courtright, Straightening vanes for flow measurement, Mechanical Engineering, 80, 71-73, (February,1958).

10. Sindt, C. F., and J.F. LaBrecque, An accuracy statement for a facility used to calibrate static pressure transducers and differential pressure transducers at high base pressure, Nat. Bur. Stand. (U.S.) Tech. Note. 1052, 1982.

11. Kel1, G. S., Density, Thermal expansivity, and compressibility of liquid water from $0^{\circ}$ to $150^{\circ} \mathrm{C}$. Correlations and tables for atmospheric pressure and saturation reviewed and expressed on 1968 temperature scale, J. Chem \& Eng Data, 20, 97-105, (1975).

12. International Standards Organization, Standard 5167, Measurements of fluid flow by means of orifice plates, nozzles and venturi tubes installed in circular cross-section conduits", ISO 5167 (1980 E), Geneva, (1980).

13. Beitler, S. R., The flow of water through orifices, The ohio State University, the Engineering Experiment Station Bulletin 89, 73pp. (May 1935). 


\section{APPENDIX I.}

Measurements of Orifice Meter Runs

The orifice meter runs were measured at NBS-Boulder, Colorado. The lengths were measured with a commercial steel tape. The inside diameters were measured with inside micrometers. Location of orifice taps and dowel pins were determined by inserting precision ground rods in the holes and measuring the distance from the rods to the flange face or flange hole with micrometers. The dowel pin location listed is the radius from the flange center line to the center line of the dowel hole. The radius used for the flange hole was the average of the four radii measured at the flange tap as shown in columins $C$ and $D$ on the following charts.

The surface finish listed for the upstream tube is, first, the finish alongside the flange tape hole and, second, the finish upstream from the flange face as far as the instrument would reach, about $12 \mathrm{~cm}$.

The orifice meters are identified with an impression stamp either on the flanges or on the pipe. Identification of the meter runs included the two leading letters FE followed by a number which identifies the meter assembly. The number is followed by a letter which identifies the meter section. The letter $A$ is used for the approach section; $B$ is used for the upstream section and $C$ is used for the downstream section. The number identification for size uses 1 and 2 for the two inch nominal meter, 3 and 4 for the three inch meter, 5 and 6 for the four inch meter and 7 and 8 for the six inch meter. An example of the identification would be FE6-A, which is for a four inch approach section. 


\section{ORIFICE METER TUBE MEASUREMENTS}

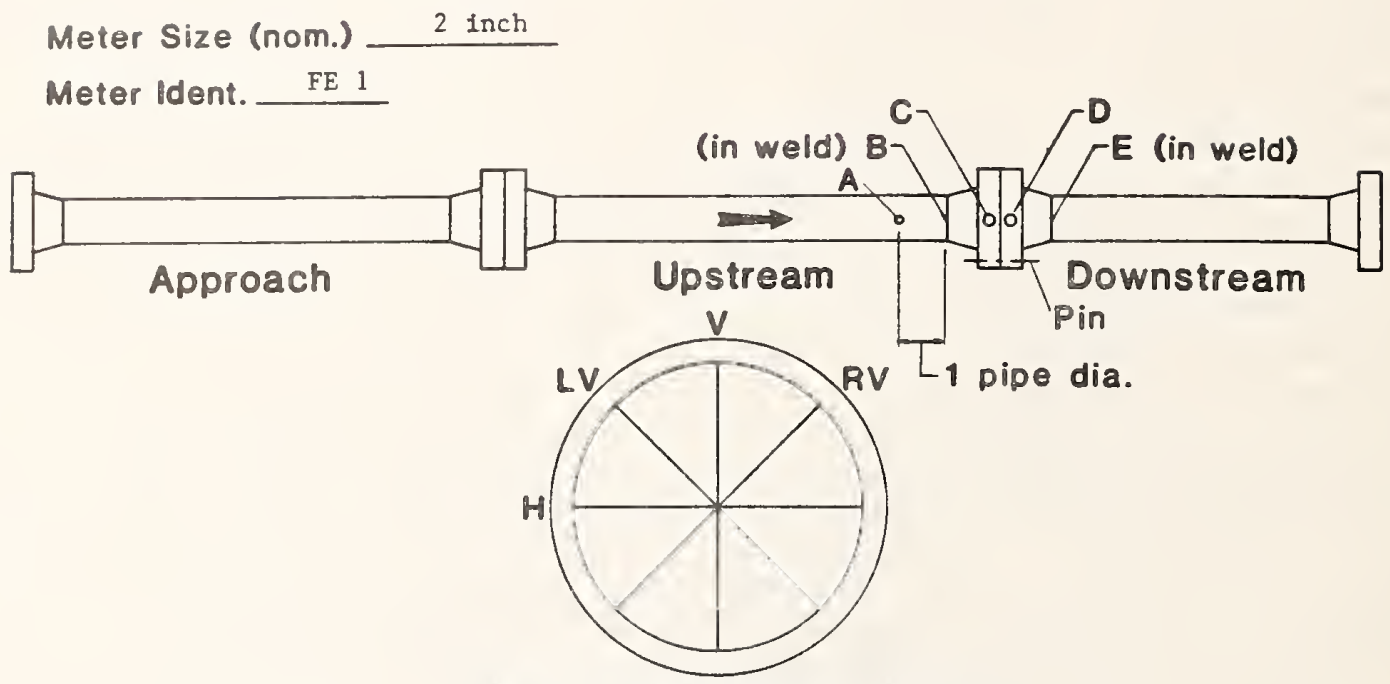

View In Direction Of Flow

\begin{tabular}{|c|c|c|c|c|c|c|c|c|c|c|}
\hline & \multicolumn{6}{|c|}{ Upstream Tube } & \multicolumn{4}{|c|}{ Downstream Tube } \\
\hline & \multicolumn{2}{|c|}{$A$} & \multicolumn{2}{|c|}{ B } & \multicolumn{2}{|c|}{ C } & \multicolumn{2}{|c|}{ D } & \multicolumn{2}{|c|}{$E$} \\
\hline & $\mathrm{cm}$ & in & $\mathrm{cm}$ & in & $\mathrm{cm}$ & in & $\mathrm{cm}$ & in & $\mathrm{cm}$ & in \\
\hline V & 5.250 & 2.067 & 5.2481 & 2.0662 & 5.2532 & 2.0682 & 5.2446 & 2.0648 & 5.2413 & 2.0635 \\
\hline LV & 5.250 & 2.067 & 5.2532 & 2.0682 & 5.2515 & 2.0675 & 5.2489 & 2.0665 & 5.2421 & 2.0638 \\
\hline RV & 5.248 & 2.067 & 5.2471 & 2.0658 & 5.2497 & 2.0668 & 5.2514 & 2.0675 & 5.2405 & 2.0632 \\
\hline $\mathrm{H}$ & 5.250 & 2.067 & 5.2489 & 2.0665 & 5.2532 & 2.0682 & 5.2551 & 2.0650 & 5.2456 & 2.0652 \\
\hline Avg. & 5.249 & 2.067 & 5.2493 & 2.0667 & 5.2519 & 2.0677 & 5.2475 & 2.0660 & 5.2424 & 2.0639 \\
\hline
\end{tabular}

Tap Location cm (in) (from flange face)

Pin Location cm (in)

$$
\begin{aligned}
& \text { Pin } 1 \\
& \text { Pin } 2
\end{aligned}
$$

Tap Diameter cm (in)

$$
\text { Top }
$$

Bottom

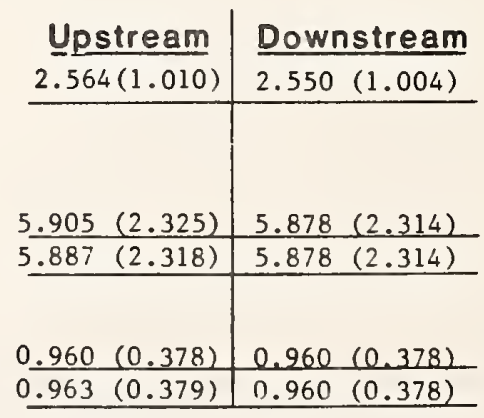

$\frac{7.0(275)}{7.5(295)}$ $177.00(69.68)$ $45.24(17.81)$ $106.52(41.93)$ 


\section{ORIFICE METER TUBE MEASUREMENTS}

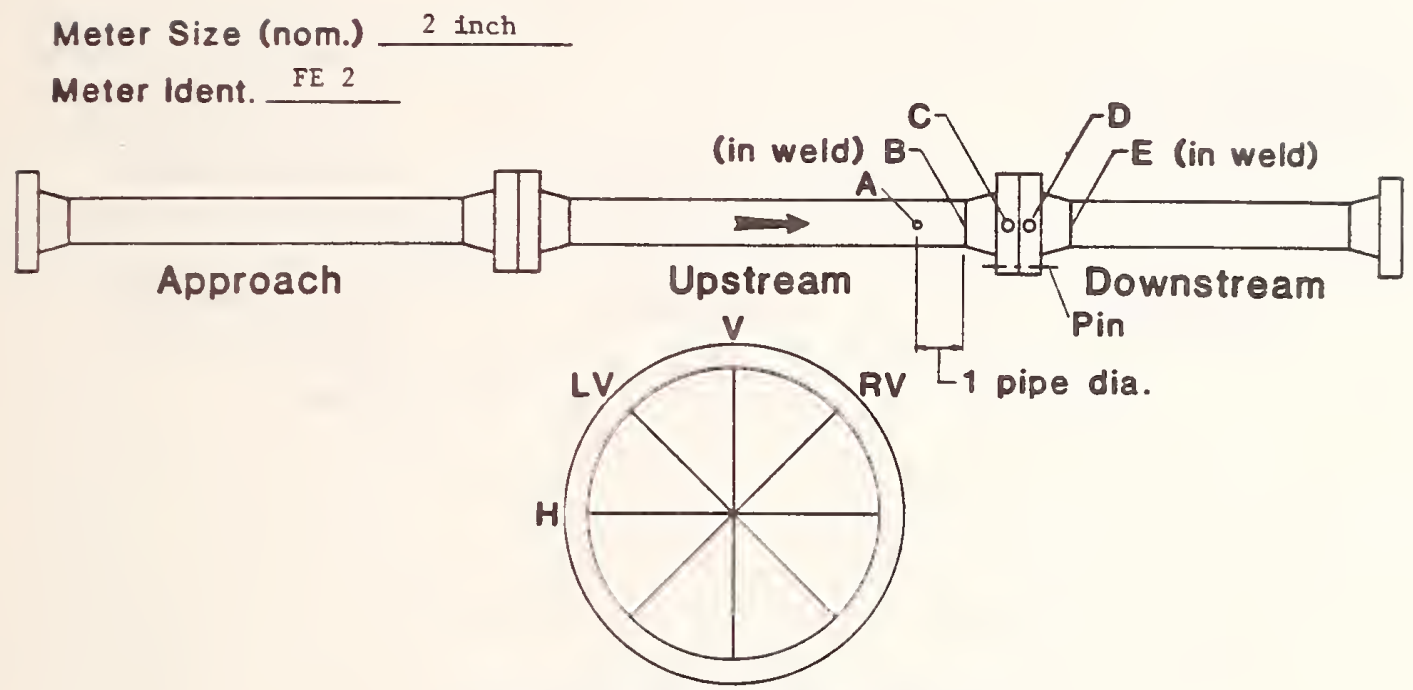

\section{View In Direction Of Flow}

\begin{tabular}{|c|c|c|c|c|c|c|c|c|c|c|}
\hline & \multicolumn{6}{|c|}{ Upstream Tube } & \multicolumn{4}{|c|}{ Downstream Tube } \\
\hline & \multicolumn{2}{|c|}{ A } & \multicolumn{2}{|c|}{$B$} & \multicolumn{2}{|c|}{ C } & \multicolumn{2}{|c|}{$D$} & \multicolumn{2}{|c|}{$E$} \\
\hline & $\mathrm{cm}$ & in & $\mathrm{cm}$ & in & $\mathrm{cm}$ & in & $\mathrm{cm}$ & in & $\mathrm{cm}$ & in \\
\hline $\bar{V}$ & 5.253 & 2.068 & 5.2608 & 2.0712 & 5.2583 & 2.0702 & 5.2456 & 2.0652 & 5.2413 & 2.0635 \\
\hline LV & 5.248 & 2.066 & 5.2497 & 2.0668 & 5.2608 & 2.0712 & 5.2476 & 2.0660 & 5.2431 & 2.0642 \\
\hline RV & 5.248 & 2.066 & 5.2608 & 2.0712 & 5.2624 & 2.0718 & 5.2431 & 2.0642 & 5.2446 & 2.0648 \\
\hline H & 5.250 & 2.067 & 5.2565 & 2.0695 & 5.2578 & 2.0700 & 5.2426 & 2.0640 & 5.2438 & 2.0645 \\
\hline Avg & 5.250 & 2.067 & 5.2570 & 2.0697 & 5.2598 & 2.0708 & 5.2447 & 2.0649 & 5.2432 & $2.06 \div 3$ \\
\hline
\end{tabular}

Tap Location cm (in)
(from flange face)

Pin Location cm (in) Pin $* 1$

Pin +2

Tap Diameter cm (in)

Top

Bottom

\begin{tabular}{c|c}
$\begin{array}{c}\text { Upstream } \\
2.565-2.569\end{array}$ & $\frac{\text { Downstream }}{2.549-2.555}$ \\
\hline $\begin{array}{l}(1.010-1.012) \\
(1.004-1.006)\end{array}$ & \\
\hline $5.901(2.323)$ & $5.899(2.322)$ \\
\hline $5.909(2.326)$ & $5.889(2.318)$ \\
\hline $0.960(0.378)$ & $0.960(0.378)$ \\
\hline $0.963(0.379)$ & $0.958(0.377)$ \\
\hline
\end{tabular}

Surface Finish $\mu \mathrm{m}$ ( $\mu$ in)

Tube Length $\mathrm{cm}$ (in)

Approach Tube

Upstream Tube

Downstream Tube $\frac{2.5(98)}{4.5(177)}$

$\begin{array}{r}177.16(69.75) \\ \hline 45.56(17.93) \\ \hline 106.52(41.93) \\ \hline\end{array}$




\section{ORIFICE METER TUBE MEASUREMENTS}

Meter Size (nom.) 3 inch

Meter Ident. FE3

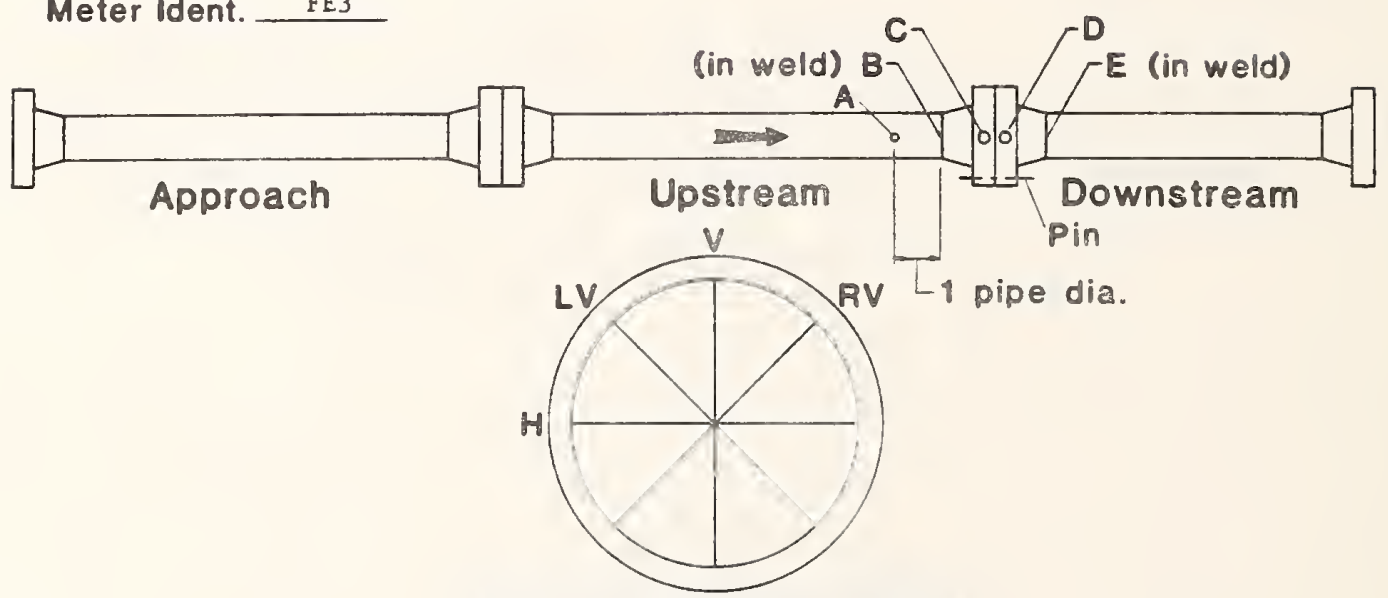

View In Direction Of Flow

\begin{tabular}{|c|c|c|c|c|c|c|c|c|c|c|}
\hline & \multicolumn{6}{|c|}{ Upstream Tube } & \multicolumn{4}{|c|}{ Downstream Tube } \\
\hline & \multicolumn{2}{|c|}{ A } & \multicolumn{2}{|c|}{ B } & \multicolumn{2}{|c|}{ C } & \multicolumn{2}{|c|}{ D } & \multicolumn{2}{|c|}{ E } \\
\hline & $\mathrm{cm}$ & in & $\mathrm{cm}$ & in & $\mathrm{cm}$ & in & $\mathrm{cm}$ & in & $\mathrm{cm}$ & in \\
\hline v & 7.798 & 3.070 & 7.7927 & 3.0680 & 7.7914 & 3.0675 & 7.7983 & 3.0702 & 7.8041 & 3.0725 \\
\hline LV & 7.803 & 3.072 & 7.7935 & 3.0683 & 7.7953 & 3.0690 & 7.7978 & 3.0700 & 7.8003 & 3.0710 \\
\hline RV & 7.785 & 3.065 & 7.7927 & 3.0680 & 7.7922 & 3.0678 & 7.8026 & 3.0719 & 7.8008 & 3.0712 \\
\hline $\mathrm{H}$ & 7.803 & 3.072 & 7.7826 & 3.0640 & 7.7915 & 3.0675 & 7.8000 & 3.0710 & 7.7914 & 3.0675 \\
\hline Avg. & 7.797 & 3.070 & 7.7904 & 3.0671 & 7.7926 & 3.0679 & 7.7991 & 3.0708 & 7.7992 & 3.0706 \\
\hline
\end{tabular}

Tap Location $\mathrm{cm}$ (in)

(from flange face)

Pin Location cm (in)

Pin 1

Pin $* 2$

Tap Diameter cm (in)

Top

Bottom

Surface Finish $\mu \mathrm{m}(\mu \mathrm{in}) \frac{2.0(78)}{4.0(157)}$

Tube Length $\mathrm{cm}$ (in)

Approach Tube

Upstream Tube

Downstream Tube

$265.91(104.68)$ $\frac{68.42(26.93)}{137.00(53.93)}$

$137.00(53.93)$

\begin{tabular}{c|c} 
Upstream & Downstream \\
\hline $2.536-2.539$ & $2.558(1.007)$ \\
\hline$(0.999-1.000)$ & \\
$7.770(3.059)$ & $7.794(3.068)$ \\
\hline $7.770(3.059)$ & $7.794(3.068)$ \\
\hline & \\
\hline $0.965(0.380)$ & $0.965(0.380)$ \\
\hline $0.963(0.379)$ & $0.963(0.379)$ \\
\hline
\end{tabular}




\section{ORIFICE METER TUBE MEASUREMENTS}

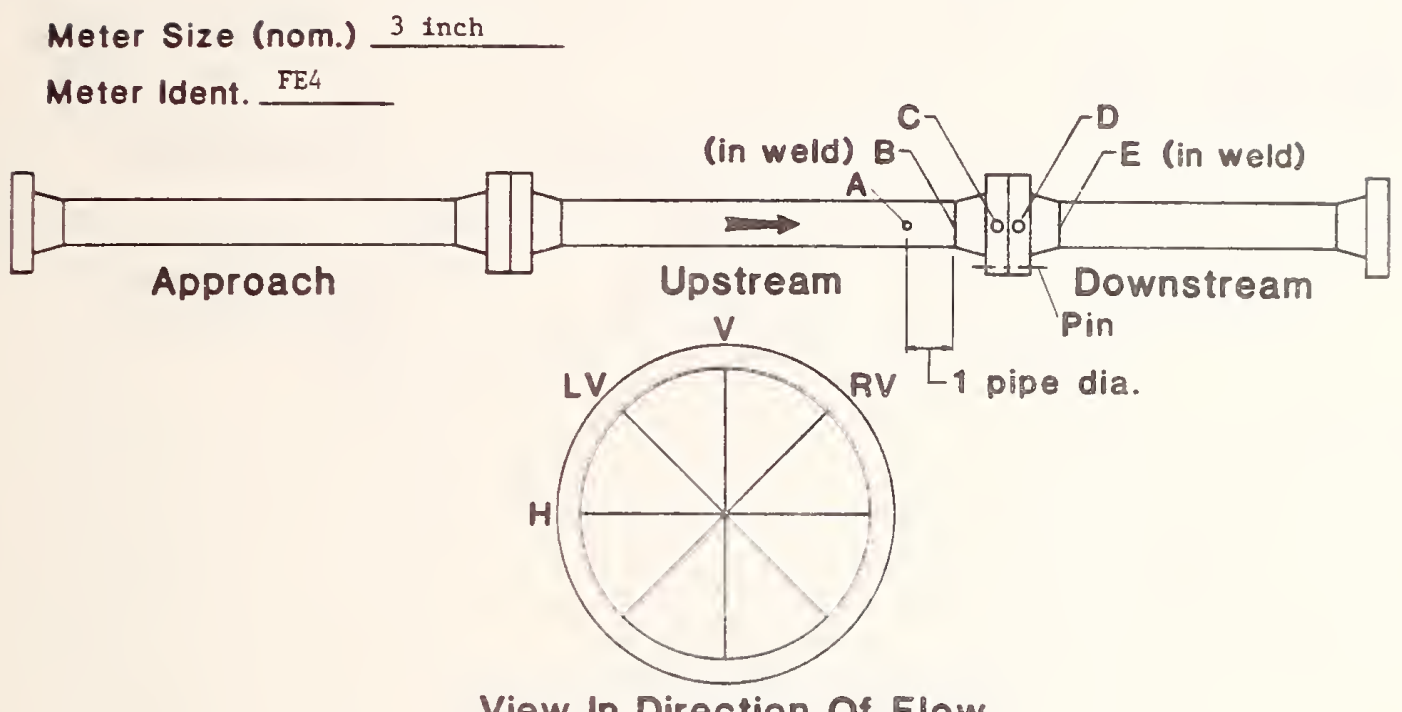

\begin{tabular}{|c|c|c|c|c|c|c|c|c|c|c|}
\hline & \multicolumn{6}{|c|}{ Upstream Tube } & \multicolumn{4}{|c|}{ Downstream Tube } \\
\hline & \multicolumn{2}{|c|}{$A$} & \multicolumn{2}{|c|}{ B } & \multicolumn{2}{|c|}{ C } & \multicolumn{2}{|c|}{ D } & \multicolumn{2}{|c|}{$E$} \\
\hline & $\mathrm{cm}$ & in & $\mathrm{cm}$ & in & $\mathrm{cm}$ & in & $\mathrm{cm}$ & in & $\mathrm{cm}$ & in \\
\hline V & 7.805 & 3.073 & 7.7214 & 3.0675 & 7.7958 & 3.0692 & 7.7998 & 3.0708 & 7.7907 & 3.0672 \\
\hline LV & 7.818 & 3.078 & 7.7838 & 3.0645 & 7.7876 & 3.0660 & 7.7978 & 3.0700 & 7.7864 & 3.0655 \\
\hline RV & 7.762 & 3.056 & 7.7864 & 3.0655 & 7.7948 & 3.0688 & 7.8003 & 3.0710 & 7.7958 & 3.0692 \\
\hline$H$ & 7.821 & 3.079 & 7.7914 & 3.0675 & 7.7902 & 3.0670 & 7.7983 & 3.0702 & 7.7889 & 3.0665 \\
\hline Avg. & 7.802 & 3.072 & 7.7383 & 3.0663 & 7.7921 & 3.0678 & 7.7991 & 3.0705 & 7.7905 & 3.0671 \\
\hline
\end{tabular}

Tap Location cm (in) (from flange face)

Pin Location cm (in)

$P$ in +1

Pin $\neq 2$

Tap Diameter cm (in)

Top

Bottom

Surface Finish $\mu \mathrm{m}(\mu$ in $) \frac{9.0(354)}{7.75(305)}$

Tube Length $\mathrm{cm}$ (in)

Approach Tube

Upstream Tube

Downstream Tube

$266.07(104.75)$

$68.74(27.06)$

$137.16(54.00)$

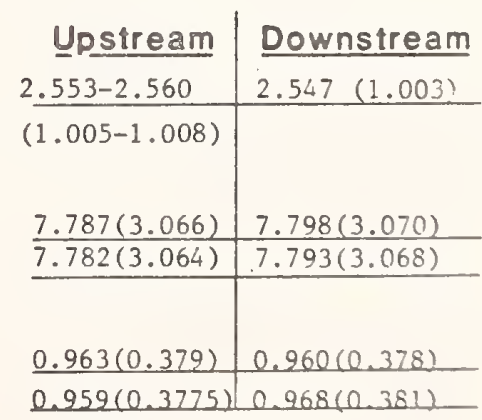




\section{ORIFICE METER TUBE MEASUREMENTS}

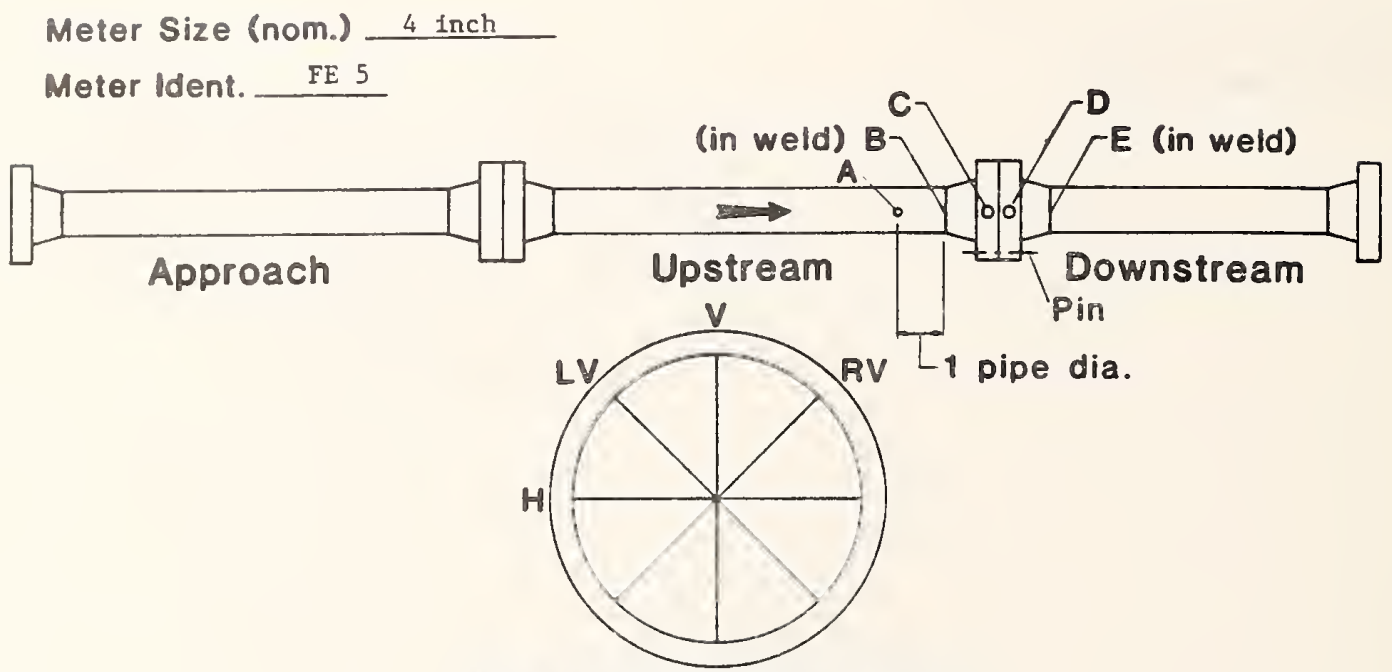

View In Direction of Flow

\begin{tabular}{|c|c|c|c|c|c|c|c|c|c|c|}
\hline & \multicolumn{6}{|c|}{ Upstream Tube } & \multicolumn{4}{|c|}{ Downstream Tube } \\
\hline & \multicolumn{2}{|c|}{ A } & \multicolumn{2}{|c|}{ B } & \multicolumn{2}{|c|}{ C } & \multicolumn{2}{|c|}{ D } & \multicolumn{2}{|c|}{$E$} \\
\hline & $\mathrm{cm}$ & in & $\mathrm{cm}$ & in & $\mathrm{cm}$ & in & $\mathrm{cm}$ & in & $\mathrm{cm}$ & in \\
\hline $\mathbf{V}$ & -- & -- & 10.2273 & 4.0265 & 10.2222 & 4.0245 & 10.2222 & 4.0245 & 10.2062 & 4.0182 \\
\hline LV & -- & - & 10.2171 & 4.0225 & 10.2108 & 4.0200 & 10.2205 & 4.0238 & 10.2095 & 4.0195 \\
\hline RV & $=$ & - & 10.2222 & 4.0245 & 10.2113 & 4.0202 & 10.2171 & 4.0225 & 10.2215 & 4.0242 \\
\hline $\mathrm{H}$ & -- & -- & 10.2210 & 4.0240 & 10.2222 & 4.0245 & 10.2184 & 4.0230 & 10.2095 & 4.0195 \\
\hline Avg. & 10.216 & 4.022 & 10.2219 & 4.0244 & 10.2166 & 4.0223 & 10.2196 & 4.0235 & 10.2117 & 4.0204 \\
\hline
\end{tabular}

Tap Location cm (in) (from flange face)

Pin Location $\mathrm{cm}$ (in)

Pin $* 1$

Pin $\$ 2$

Tap Diameter $\mathrm{cm}$ (in)

Top

Bottom

Surface Finish $\mu \mathrm{m}(\mu$ in $)$

Tube Length $\mathrm{cm}$ (in)

Approach Tube

Upstream Tube

Downstream Tube

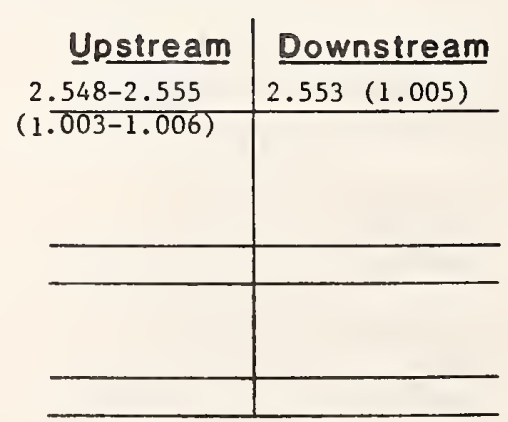

$2.5-5.0(98-197)$

$\begin{array}{r}355.12(139.81) \\ \hline 91.44(36.00) \\ \hline 167.48(65.93)\end{array}$




\section{ORIFICE METER TUBE MEASUREMENTS}

Meter Size (nom.) 4 inch

Meter Ident. FE 6
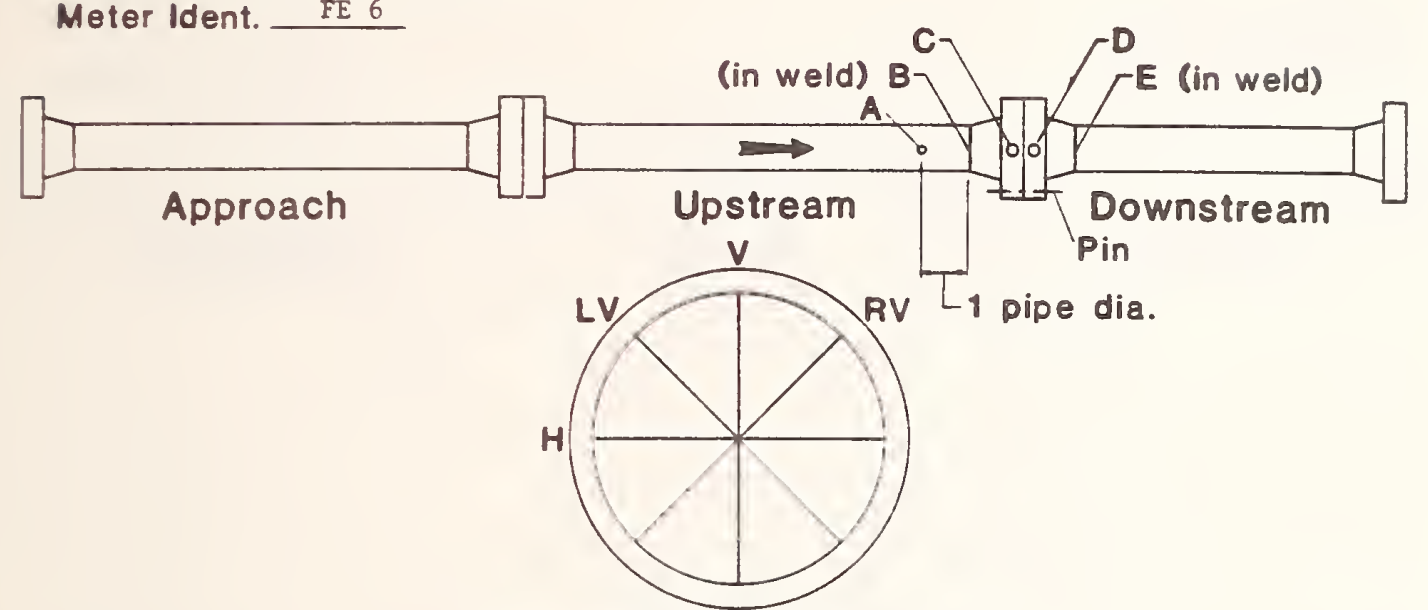

View In Direction of Flow

\begin{tabular}{|c|c|c|c|c|c|c|c|c|c|c|}
\hline & \multicolumn{6}{|c|}{ Upstream Tube } & \multicolumn{4}{|c|}{ Downstream Tube } \\
\hline & \multicolumn{2}{|c|}{ A } & \multicolumn{2}{|c|}{$B$} & \multicolumn{2}{|c|}{ C } & \multicolumn{2}{|c|}{$D$} & \multicolumn{2}{|c|}{$E$} \\
\hline & $\mathrm{cm}$ & in & $\mathrm{cm}$ & in & $\mathrm{cm}$ & in & $\mathrm{cm}$ & in & $\mathrm{cm}$ & in \\
\hline $\mathbf{V}$ & 10.236 & 4.030 & 10.2265 & 4.0262 & 10.2349 & 4.0295 & 10.2248 & 4.0255 & 10.2184 & 4.0230 \\
\hline LV & 10.229 & 4.027 & 10.2331 & 4.0288 & 10.2222 & 4.0245 & 10.2235 & 4.0250 & 10.2095 & 4.0195 \\
\hline RV & 10.234 & 4.029 & 10.2248 & 4.0255 & 10.2204 & 4.0238 & 10.2306 & 4.0278 & 10.2342 & 4.0292 \\
\hline $\mathrm{H}$ & 10.234 & 4.027 & 10.2306 & 4.0278 & 10.2265 & 4.0262 & 0.2215 & 4.0242 & 10.2159 & 4.0220 \\
\hline Avg. & 10.233 & 4.028 & 10.2288 & 4.0271 & 10.2260 & 4.0260 & 10.2222 & 4.0256 & 10.2195 & 4.0234 \\
\hline
\end{tabular}

Tap Location cm (in)

(from flange face)

Pin Location cm (in)

Pin +1

Pin $\neq 2$

Tap Diameter cm (in)
Top
Bottom

Surface Finish $\mu \mathrm{m}(\mu \mathrm{in})$

Tube Length $\mathrm{cm}$ (in)

Approach Tube

Upstream Tube

Downstream Tube

\begin{tabular}{c|c}
$\frac{\text { Upstream }}{2.553-2.560}$ & $\frac{\text { Downstream }}{2.550-2.555}$ \\
\hline$(1.005-1.008)$ & $(1.004-1.006)$ \\
& \\
\hline $.505(3.742)$ & $9.517(3.747)$ \\
\hline $9.492(3.737)$ & $9.568(3.767)$ \\
\hline $1.273(.501)$ & $1.295(.510)$ \\
\hline $1.271(.502)$ & $1.290(.508)$ \\
\hline
\end{tabular}




\section{ORIFICE METER TUBE MEASUREMENTS}

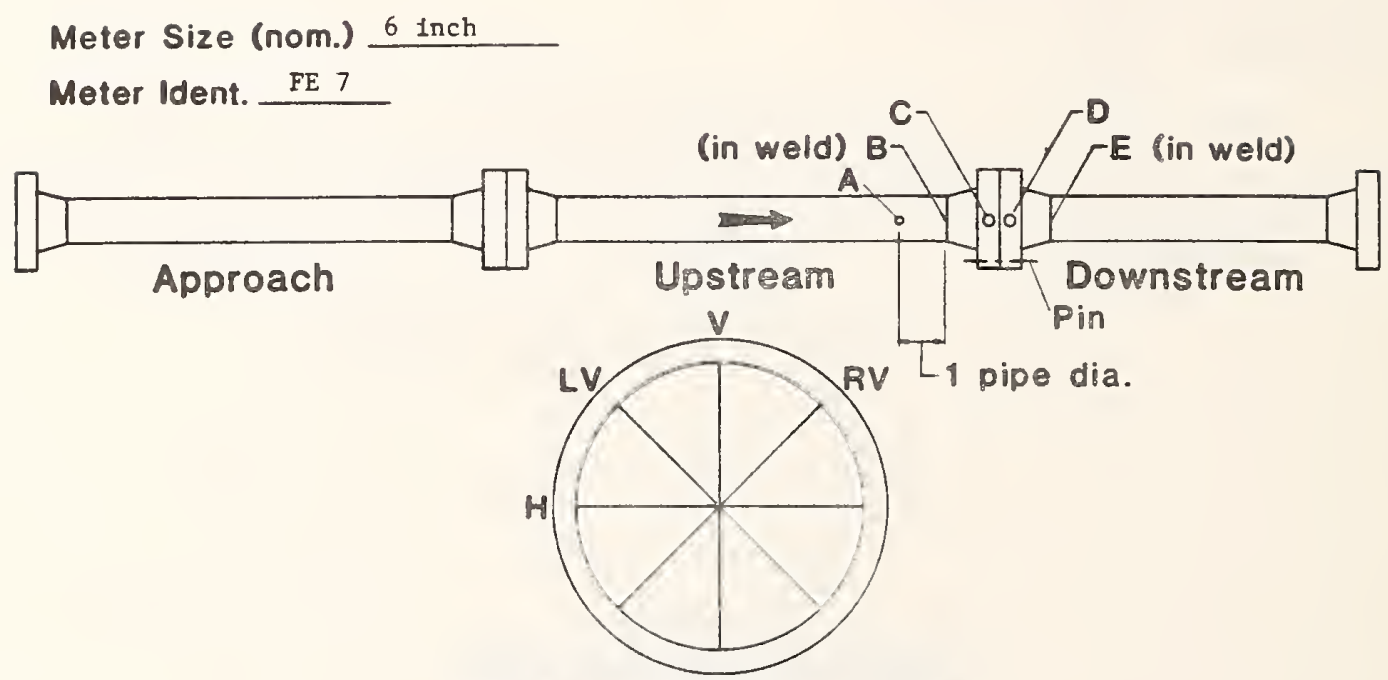

View in Direction of Flow

\begin{tabular}{|c|c|c|c|c|c|c|c|c|c|c|}
\hline & \multicolumn{6}{|c|}{ Upstream Tube } & \multicolumn{4}{|c|}{ Downstream Tube } \\
\hline & \multicolumn{2}{|c|}{ A } & \multicolumn{2}{|c|}{ B } & \multicolumn{2}{|c|}{ C } & \multicolumn{2}{|c|}{0} & \multicolumn{2}{|c|}{$E$} \\
\hline & $\mathrm{cm}$ & in & $\mathrm{cm}$ & in & $\mathrm{cm}$ & in & $\mathrm{cm}$ & in & $\mathrm{cm}$ & in \\
\hline $\mathbf{V}$ & - & -- & 15.4224 & 6.0718 & 15.4165 & 6.0695 & 15.4148 & 6.0688 & 15.4285 & 6.0742 \\
\hline LV & - & -- & 15.4249 & 6.0728 & 15.4178 & 6.0700 & 15.4155 & 6.0691 & 15.4432 & 6.0800 \\
\hline RV & -- & -- & 15.4178 & 6.0700 & 15.4178 & 6.0700 & 15.4140 & 6.0685 & 15.4292 & 6.0745 \\
\hline$H$ & -- & -- & 15.4229 & 6.0720 & 15.4173 & 6.0698 & 15.4153 & 6.0690 & 15.4407 & 6.0790 \\
\hline Avg. & 15.433 & 6.076 & 15.4220 & 6.0717 & 15.4174 & 6.0698 & 15.4149 & 6.0689 & 15.4354 & 6.0769 \\
\hline
\end{tabular}

Tap Location $\mathrm{cm}$ (in) (from flange face)

Pin Location cm (in)

Pin +1

Pin +2

Tap Diameter cm (in) Top Bottom
Surface Finish $\mu \mathrm{m}(\mu \mathrm{in})$

Tube Length $\mathrm{cm}$ (in) Approach Tube Upstream Tube Downstream Tube

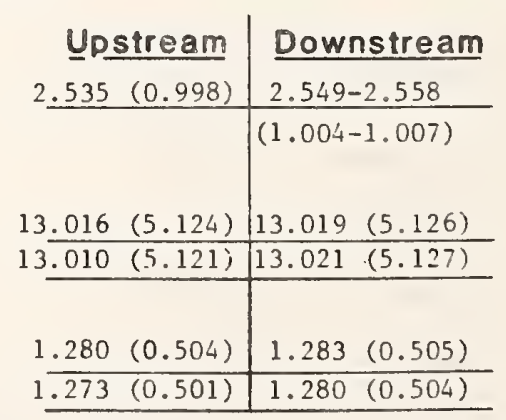

\begin{tabular}{ll}
531.34 & $(209.18)$ \\
\hline 137.16 & $(54.0)$ \\
\hline 228.44 & $(89.93)$ \\
\hline
\end{tabular}




\section{ORIFICE METER TUBE MEASUREMENTS}

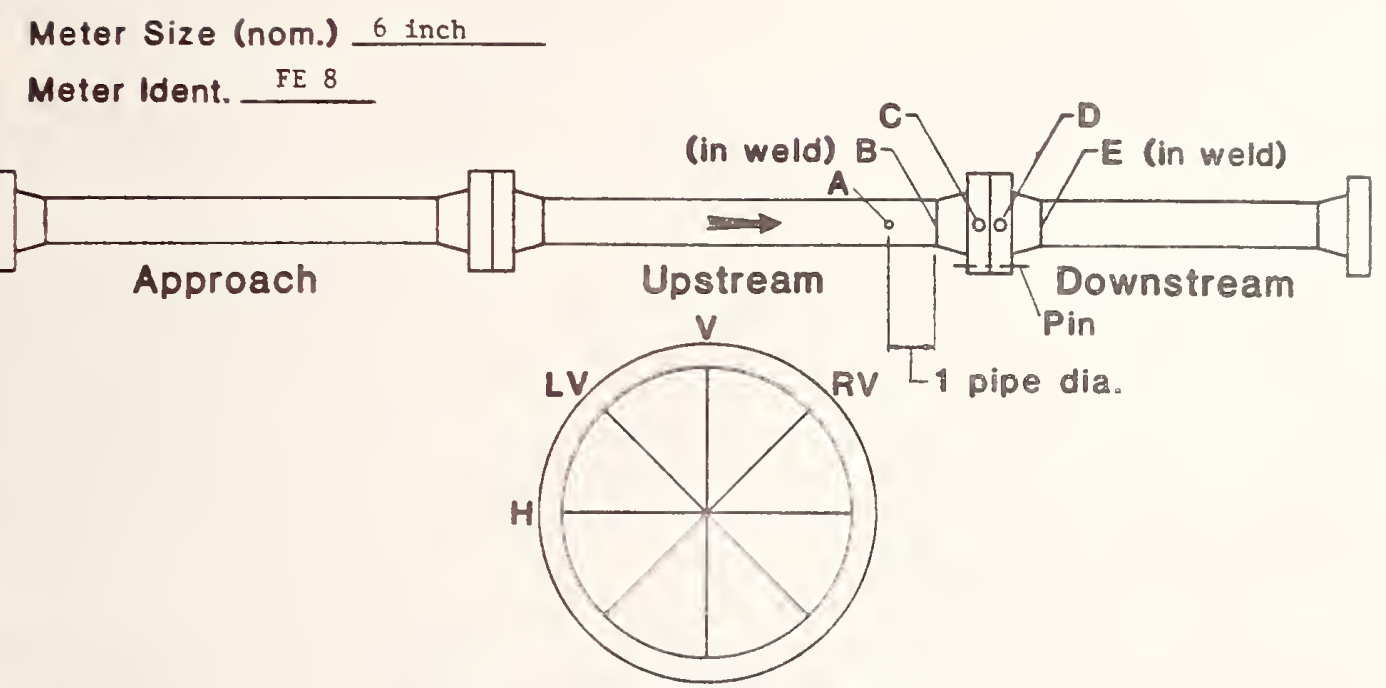

View In Direction Of Flow

\begin{tabular}{|c|c|c|c|c|c|c|c|c|c|c|}
\hline & \multicolumn{6}{|c|}{ Upstream Tube } & \multicolumn{4}{|c|}{ Downstream Tube } \\
\hline & \multicolumn{2}{|c|}{ A } & \multicolumn{2}{|c|}{ B } & \multicolumn{2}{|c|}{ C } & \multicolumn{2}{|c|}{$D$} & \multicolumn{2}{|c|}{$E$} \\
\hline & $\mathrm{cm}$ & in & $\mathrm{cm}$ & in & $\mathrm{cm}$ & in & $\mathrm{cm}$ & in & $\mathrm{cm}$ & in \\
\hline$\overline{\mathbf{V}}$ & 15.471 & 6.091 & 15.4325 & 6.0758 & 15.4153 & 6.0690 & 15.4107 & 6.0672 & 15.4165 & 6.0695 \\
\hline LV & 15.474 & 6.092 & 15.4224 & 6.0718 & 15.4165 & 6.0695 & 15.4081 & 6.0662 & 15.4457 & 6.0810 \\
\hline RV & 15.469 & 6.090 & 15.4259 & 6.0732 & 15.4148 & 6.0688 & 15.4076 & 6.0660 & 15.4254 & 6.0730 \\
\hline $\mathrm{H}$ & 15.474 & 6.092 & 15.4234 & 6.0722 & 15.4173 & 6.0698 & 15.4076 & 6.0660 & 15.4381 & 6.0780 \\
\hline Avg. & 15.472 & 6.091 & 15.4261 & 6.0733 & 15.4160 & 6.0693 & 15.4085 & 6.0664 & 15.4314 & 6.0754 \\
\hline
\end{tabular}

Tap Location cm (in)

(from flange face)

Pin Location $\mathrm{cm}$ (in)

$P$ in 1

Pin 2

Tap Diameter cm (in)

Top

Bottom

Surface Finish $\mu \mathrm{m}(\mu$ in $)$

Tube Length $\mathrm{cm}$ (in)

Approach Tube

Upstream Tube

Downstream Tube

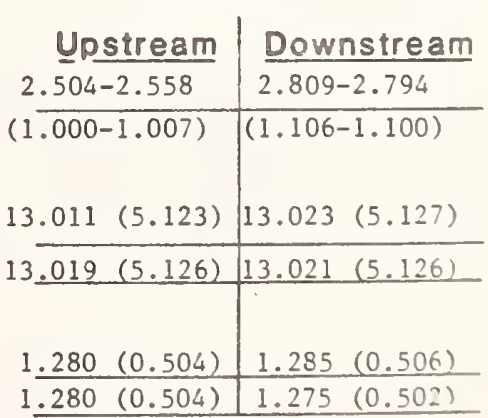

$\frac{5.5(216)}{5.5(216)}$

$531.50(209.25)$

\begin{tabular}{ll}
$137.00(53.93)$ \\
\hline $228.44(89.93)$
\end{tabular} 
Measurements of Orifice Plates

The orifice plates were measured at NBS-Boulder, Colorado. The diameters and the concentricty of the orifice to the plate outside diameter were measured with an optical measuring device. The outside diameter was first determined then the center of the plate was calculated. From this center the orifice diameter was measured as indicated and the orifice center was then determined relative to the plate outside diameter. The uncertainty in the outside diameter measurement is estimated to be $0.03 \mathrm{~mm}$ therefore the uncertainty in the concentricity is also $0.03 \mathrm{~mm}$. No concentricity measurements were made on the plates of the FE5/6 B group. These measurements wi11 be made at a later date by NBS-Washington.

The surface finish was measured across and with the visible finishing marks and are identified as "across" and "with" respectively. "Plate flatness was checked with a straight edge and a feeler gauge. The straight edge was laid across the upstream face at $45^{\circ}$ intervals. At each position the inspector attempted to slip a $.025 \mathrm{~mm}$ feeler gauge between the plate and the straight edge. If the gauge went between the straight edge and the plate the deviation from $.025 \mathrm{~mm}$ was noted as plate flatness, otherwise it was listed as "0.K.". The "orifice edge" was inspected by making a mold of the edge at one place and viewing a cross-section of this mold at 100 times magnification. This permitted determining if the apparent radius of the "sharp" edae was greater than $0.025 \mathrm{~mm}$. If the radius was larger or included burrs, it was noted, otherwise it was listed as "O.K." General inspection of the plates were made to note nicks at the edge or in the upstream face within the pipe diameter. If nicks were visible, they were noted under comments. Position of the blemishes are in degrees clockwise from the handle viewed from the inlet side of the plate. For 24 hours prior to measurement, all of the plates were placed in a room which was controlled at $293.15 K+0.1 K$. The orifice plates were all beveled on the downstream side with $\mathrm{a}^{-} 45^{\circ}$ bevel. The following figure shows how the bevel was measured and where the edge thickness " $e$ " was measured.

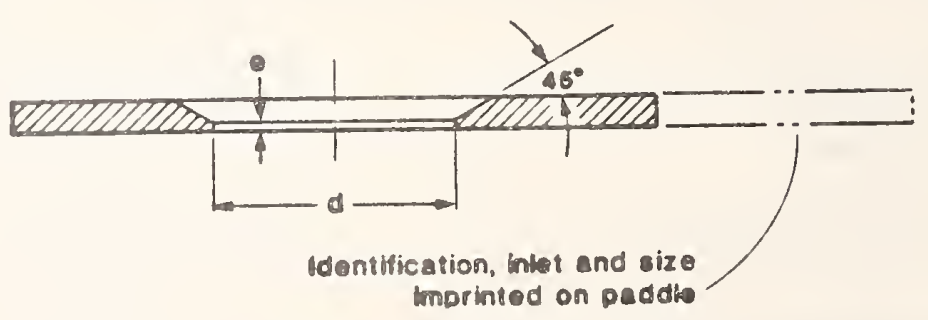

The orifice plates were all identified using a system comparable to that used for the orifice meter runs. The two leading letters FE were followed by two numbers separated with a slash. These numbers specified the meter runs that accommodate this plate. Following these numbers was a dash and a number and a letter $A$ or $B$. The number was unique for the orifice hole and the letter $A$ or B specified the plate set. An example would be FE5/6-2A which is a plate for the four inch meter runs and is the 1-1/2 inch nominal orifice from the $A$ set. The identification and the nominal size are stamped on the inlet side of the handle of the orjfice plate along with the notation "INLET". 


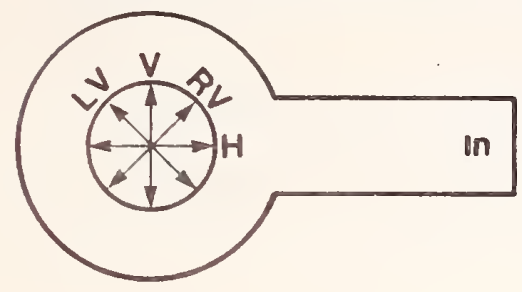

PLATE NUMBER FE $1 / 2 \quad 1 \mathrm{~A}$

\begin{tabular}{|c|c|c|}
\hline Edge Thickness, "e" (cm(in )) $0.076(0.030)$ & V & $(\mathrm{cm}($ in $)) 1.2697(0.4999)$ \\
\hline Plate Flatness & LV & $(\mathrm{cm}($ in $)) 1.2672(0.4989)$ \\
\hline late Finish $(\overline{\mu(\mu i n))}$ & RV & $(\mathrm{cm}($ in $)) 1.2687(0.4995)$ \\
\hline $0.25(9.84)$ & $\mathrm{H}$ & $(\mathrm{cm}($ in $)) 1.2659(0.4984)$ \\
\hline $0.20(7.87)$ & AVG & $(\mathrm{cm}($ in $)) \overline{1.2677(0.4991)}$ \\
\hline
\end{tabular}

Orifice Edge OK

Plate Thickness (cm(in )) $0.315(0.124)$

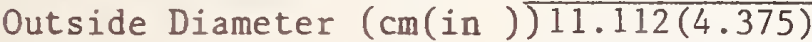

Concentricity of hole to $\mathrm{OD}(\mathrm{cm}($ in $)) \pm 0.008(0.003)$

Comments

PLATE NUMBER FE $1 / 2 \quad 2 \mathrm{~A}$

Edge Thickness, "e" (cm(in)) $0.088(0.0345)$

Plate Flatness

Plate Finish $(\mu(\mu i n))$

Across $0.25(9.84)$

With $0.25(9.84)$

Orifice Edge OK

Plate Thickness (cm(in ) ) $0.310(0.122)$

Outside Diameter ( $\mathrm{cm}$ (in) $11.111(4.373)$

Concentricity of hole to $\mathrm{OD}(\mathrm{cm}($ in $))+0.010(0.004)$

Comments

PLATE NUMBER

Edge Thickness, "e" (cm(in ) $0.080(0.0315)$

Plate Flatness

Plate Finish $(\mu(\mu$ in $))$

Across $0.20 \quad(7.87)$

With $0.20(7.87)$

Orifice Edge

Plate Thickness (cm(in))

OK

$0.310(0.122)$

Outside Diameter ( $\mathrm{cm}($ in $)) 11.107(4.373)$

Concentricity of hole to OD ( $\overline{\mathrm{cm}(\text { in }))+0.005}(0.002)$

Comments

\begin{tabular}{|c|c|}
\hline V & $(\mathrm{cm}($ in $)) 1.9035(0.7494)$ \\
\hline LV & $(\mathrm{cm}($ in $)) 1.9050(0.7500)$ \\
\hline RV & $(\mathrm{cm}($ in $)) 1.9037(0.7495)$ \\
\hline $\mathrm{H}$ & $(\mathrm{cm}($ in $)) 1.9027(0.7491)$ \\
\hline AVG & $(\mathrm{cm}($ in $)) 1.9037(0.7495)$ \\
\hline
\end{tabular}

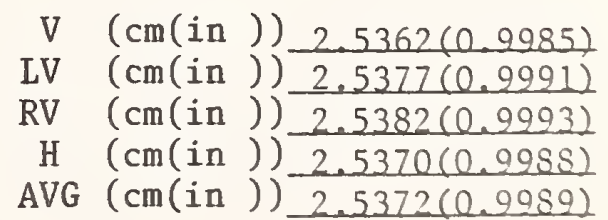

$\mathrm{V} \quad(\mathrm{cm}($ in $))-2.5362(0.9985)$

LV (cm(in ) $2.5377(0.9291)$

$\mathrm{H} \quad(\mathrm{cm}$ (in ) $2.5370(0.998 \mathrm{~s})$

AVG (cm(in ) ) $2.5372(0.9989)$ 


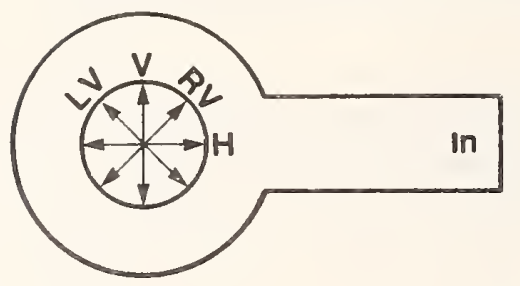

PLATE NUMBER FE $1 / 2 \quad 4 \mathrm{~A}$

Edge Thickness, "e" (cm(in )) $0.0724(0.0285)$

Plate Flatness $\mathrm{OK}$

Plate Finish $(\mu(\mu i n))$

Across

With

$0.70 \quad(27.56)$

Orifice Edge $0.25(9.84)$

Plate Thickness (cm(in )) $0.315 \quad(0.124)$

Outside Diameter (cm(in ) ) $11.107(4.373)$

Concentricity of hole to $\mathrm{OD}(\mathrm{cm}($ in $)) \quad 0.008(0.003)$

Comments
PLATE NUMBER FE $1 / 2 \quad 5 \mathrm{~A}$

Edge Thickness, "e" (cm(in )) $0.071(0.028)$

Plate Flatness

Plate Finish $(\mu(\mu i n))$

Across $0.35(13.78)$

With $0.20(7.87)$

Orifice Edge OK

Plate Thickness (cm(in )) $0.3111(0.1225)$

Outside Diameter ( $\mathrm{cm}$ (in )) $11.107(4.373)$

Concentricity of hole to oD (cm(in)) $\quad 0.005 \quad(0.002)$

Comments

PLATE NUMBER FE $1 / 2$ 6A
Edge Thickness, "e" (cm(in )) $0.075(0.030) \ldots$
Plate Flatness
Plate Finish $(\mu(\mu$ in $))$
Across $0.25 \quad(9.84)$
With $0.40(15.75)$
Orifice Edge OK $\mathrm{OK}$
Plate Thickness ( $\mathrm{cm}$ (in ))
Outside Diameter ( $\mathrm{cm}$ (in ) $11.105(4.372)$
Concentricity of hole to oD (cm(in) $\quad 0.010(0.004)$
Comments

$$
\begin{aligned}
V & (\mathrm{~cm}(\text { in })) \\
\text { LV } & (\mathrm{cm}(\text { in })) \frac{3.8108(1.5003)}{3.8113(1.5005)} \\
\text { RV } & (\mathrm{cm}(\text { in })) \frac{3.8105(1.5002)}{3.8108(1.5003)} \\
\text { H } & (\mathrm{cm}(\text { in })) \\
\text { AVG } & (\mathrm{cm}(\text { in }))
\end{aligned}
$$

$\begin{aligned} V & (\mathrm{~cm}(\text { in })) \frac{3.4917(1.3747)}{3.4928(1.3751)} \\ \text { LV } & (\mathrm{cm}(\text { in })) \\ R V & (\mathrm{~cm}(\text { in })) \frac{3.4915(1.3746)}{3.4912(1.3745)} \\ H & (\mathrm{~cm}(\text { in })) \\ \text { AVG } & (\mathrm{cm}(\text { in }))\end{aligned}$

$\mathrm{VV} \quad(\mathrm{cm}(\mathrm{in})) \frac{3.4917(1.3747)}{3.4928(1.3751)}$

$\mathrm{RV} \quad(\mathrm{cm}$ (in )) $3.4915(1.3746)$

AVG (cm(in ) $3.4917(1.3747)$

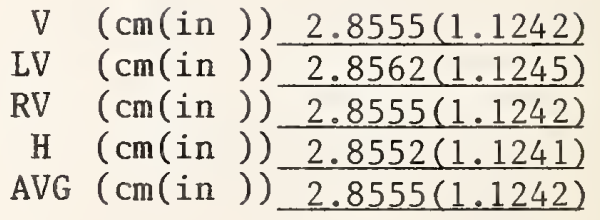




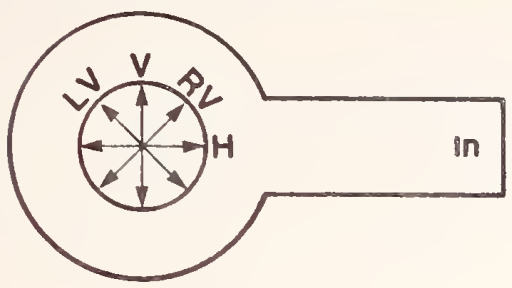

PLATE NUMBER FE $1 / 2 \quad 1 B$

\begin{tabular}{|c|c|c|}
\hline Edge Thickness, "e" (cm(in & V & $(\mathrm{cm}($ in $)) 1.2675(0.4990)$ \\
\hline Plate Flatness & LV & $(\mathrm{cm}($ in $)) 1.2738(0.5015)$ \\
\hline Plate Finish $(\mu(\mu i n))$ & RV & $(\mathrm{cm}($ in $)) 1.2710(0.5004)$ \\
\hline $0.20(7.87)$ & $\mathrm{H}$ & $(\mathrm{cm}($ in $)) 1.2659(0.4984)$ \\
\hline $0.25 \quad(9.84)$ & AVG & $(\mathrm{cm}($ in $)) 1.2695(0.4998)$ \\
\hline
\end{tabular}

\section{Orifice Edge OK}

Plate Thickness (cm(in )) $0.302(0.119)$

Outside Diameter ( $\mathrm{cm}$ (in ) $11.100(4.370)$

Concentricity of hole to OD ( $\mathrm{cm}($ in $)) \underline{+0.025}(0.010)$

Comments

PLATE NUMBER FE $1 / 2$ 2B

\begin{tabular}{|c|c|c|}
\hline Edge Thickness, "e" & V & $(\mathrm{cm}($ in $)) 1.9040(0.7496)$ \\
\hline Plate Flatness & LV & $(\mathrm{cm}($ in $)) 1.9047(0.7499)$ \\
\hline Plate Finish $(\mu(\mu$ in $))$ & RV & $(\mathrm{cm}($ in $)) 1.9045(0.7498)$ \\
\hline $0.25 \quad(9.84)$ & $\mathrm{H}$ & $(\mathrm{cm}($ in $)) 1.9045(0.7498)$ \\
\hline $0.20(7.87)$ & AVG & $(\mathrm{cm}($ in $)) 1.9045(0.7498)$ \\
\hline
\end{tabular}

\section{Orifice Edge OK}

Plate Thickness (cm(in)) $0.3111(0.1225)$

Outside Diameter ( $\mathrm{cm}$ (in ) $11.105(4.372)$

Concentricity of hole to OD ( cm(in ) $+0.005(0.002)$

Comments

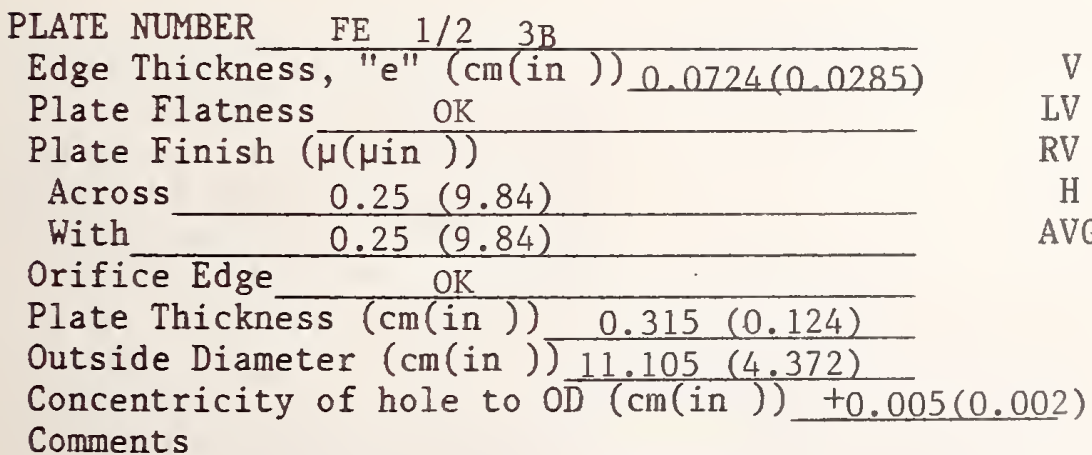

\begin{tabular}{|c|c|}
\hline$(\mathrm{cm}$ (in & )) $2.5390(0.9996)$ \\
\hline$(\mathrm{cm})$ in & )$\longdiv { 2 . 5 3 9 2 ( 0 . 9 9 9 7 ) }$ \\
\hline$(\mathrm{cm}$ ( in & $2.5395(0.9998)$ \\
\hline (cm $($ in & $2.5397(0.9995)$ \\
\hline$(\mathrm{cm})$ in & )$) 2.5390(0.9996)$ \\
\hline
\end{tabular}




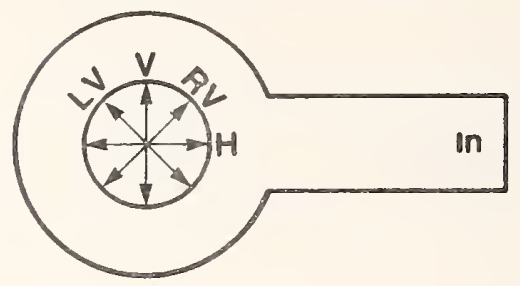

PLATE NUMBER FE $1 / 2$ 4B
Edge Thickness, "e" (cm(in ) $0.0737(0.0290)$

Plate Flatness OK

Plate Finish $(\overline{\mu(\mu i n)})$

Across $0.25 \quad(9.84)$

With $0.20(7.87)$

Orifice Edge OK

Plate Thickness (cm(in ) ) $0.3137 \quad(0.1235)$

\begin{tabular}{|c|c|}
\hline V & $(\mathrm{cm}$ (in ) ) $2.8567(1.1247)$ \\
\hline $\mathrm{LV}$ & $2.8570(1.1248)$ \\
\hline RV & $2.8560(1.1244)$ \\
\hline $\mathrm{H}$ & $2.8560(1.1244)$ \\
\hline VC & in $) \longdiv { 2 . 8 5 6 5 ( 1 . 1 2 4 6 ) }$ \\
\hline
\end{tabular}

Outside Diameter ( $\mathrm{cm}$ (in ) $\longdiv { 1 1 . 1 0 7 \quad ( 4 . 3 7 3 ) }$

Concentricity of hole to oD ( $\mathrm{cm}($ in $)) \pm 0.005(0.002)$

Comments

PLATE NUMBER FE $1 / 2 \quad 5 B$

Edge Thickness, "e" (cm(in)) $0.0851 \quad(0.0335)$

Plate Flatness OK

Plate Finish $(\mu(\mu$ in $))$

Across $0.25 \quad(9.84)$

With

$0.35 \quad(13.78)$

Orifice Edge OK

Plate Thickness (cm(in))

Outside Diameter (cm(in ) $11.110(4.374)$

Concentricity of hole to $0 \bar{D}(\mathrm{~cm}($ in $)) \pm 0.005 \quad(0.002)$

Comments

$\begin{aligned} & V(\mathrm{~cm}(\text { in })) \\ & \mathrm{LV}(\mathrm{cm}(\text { in })) \frac{3.4917(1.3747)}{3.4922(1.3749)} \\ & \mathrm{RV}(\mathrm{cm}(\text { in })) \\ & \mathrm{H}(\mathrm{cm}(\text { in })) \\ & \mathrm{AVG}(\mathrm{cm}(\text { in })) \\ & \frac{3.4912(1.3745)(1.3744)}{3.4915(1.3746)}\end{aligned}$

$\mathrm{V} \quad(\mathrm{cm}(\mathrm{in})) 3.4917(1.3747)$

RV (cm(in ) $\frac{3.4912(1.3745)}{3.4910(1.3744)}$

AVG (cm(in )) $3.4915(1.3746)$

PLATE NUMBER FE $1 / 2 \quad 6 B$

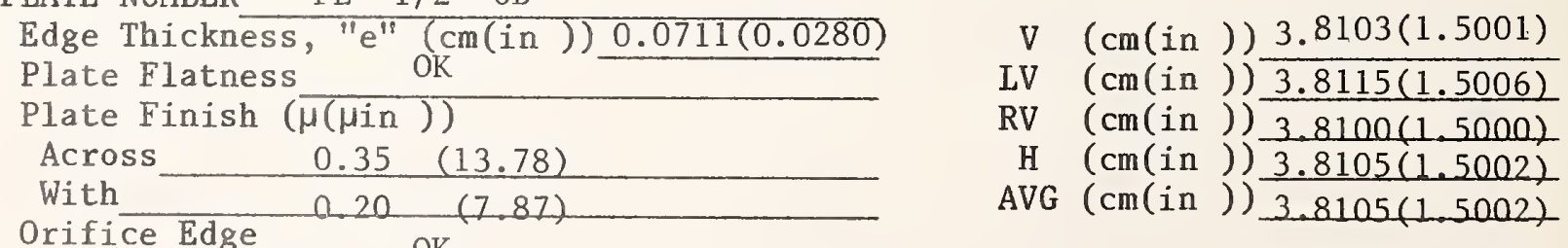

Orifice Edge _. OK

Plate Thickness (cm(in )) $0.312 \quad\left(\begin{array}{lll}0 & 123\end{array}\right.$

Outside Diameter (cm(in ) 11.107 (4.373)

Concentricity of hole to OD ( $\mathrm{cm}($ in $)) \pm 0.005(0.002)$

Comments 


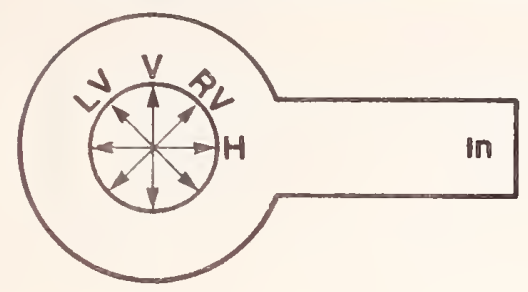

PLATE NUMBER FE $3 / 4 \quad 1 \mathrm{~A}$

Edge Thickness, "e" (cm(in )) $0.0635(0.0250)$

Plate Flatness high $0.005 \mathrm{~cm}$

Plate Finish $(\mu(\mu i n))$

Across $\quad 0.35 \quad(13.78)$

With $0.25 \quad(9.84)$

Orifice Edge OK

Plate Thickness (cm(in )) $0.3188(0.1255)$

Outside Diameter ( $\mathrm{cm}$ (in ) 14.796 (5.825)

Concentricity of hole to OD ( cm(in ) $0.008(0.003)$

Comments

PLATE NUMBER

Edge Thickness, "e" (cm(in )) $0.0549(0.0216)$

Plate Flatness low $0.010 \mathrm{~cm}$

Plate Finish $(\mu(\mu i n))$

Across

$0.35 \quad(13.78)$

With

Orifice Edge

$0.25 \quad(9.84)$

$\begin{aligned} V & (\mathrm{~cm}(\text { in })) \\ \mathrm{LV} & (\mathrm{cm}(\text { in })) \\ \mathrm{RV} & (\mathrm{cm}(\text { in })) \frac{1.5867(0.6247)}{1.5872(0.6249)} \\ \mathrm{H} & (\mathrm{cm}(\text { in })) \frac{1.5880(0.6252)}{1.585(0.6250)} \\ \text { AVG } & (\mathrm{cm}(\text { in }))\end{aligned}$

Plate Thickness (cm(in )) $0.295-0.302(0.116-0.119)$

Outside Diameter ( $\mathrm{cm}$ (in ) ) $14.9108(5.8704)$

Concentricity of hole to $\mathrm{OD}(\mathrm{cm}($ in $)) 0.008(0.003)$

Comments

PLATE NUMBER FE $3 / 4 \quad 3 \mathrm{~A}$

Edge Thickness, "e" (cm(in )) $0.0737(0.0290)$

Plate Flatness 10w $0.010 \mathrm{~cm}$

Plate Finish $(\mu(\mu i n))$

Across $\quad 0.35 \quad(13.78)$

With

Orifice Edge

$0.20 \quad(7.87)$

Plate Thickness (cm(in ) $0.3175(0.1250)$

Outside Diameter ( $\mathrm{cm}$ (in )) 14.9136(5.867)

Concentricity of hole to $\mathrm{OD}(\mathrm{cm}$ (in ) $\quad 0.005(0.002)$

Comments

$\begin{aligned} V & (\mathrm{~cm}(\text { in })) \\ \operatorname{LV} & (\mathrm{cm}(\text { in })) \\ \operatorname{RV} & (\mathrm{cm}(\text { in })) \\ \mathrm{H} & (\mathrm{cm}(\text { in })) \\ \frac{2.8575(1.1250)}{2.8582(1.1252)} & \frac{2.1253)}{2.8575(1.1250)}\end{aligned}$




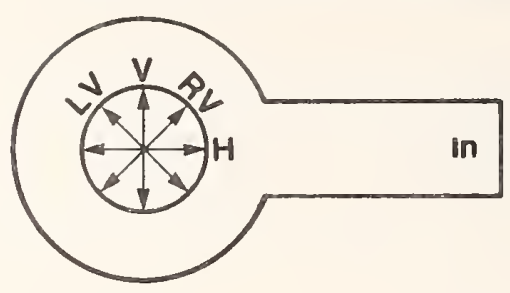

PLATE NUMBER FE $3 / 4 \quad 4 \mathrm{~A}$

Edge Thickness, "e" (cm(in )) $0.053(0.021)$

\begin{tabular}{|c|c|}
\hline ( $\mathrm{cm}$ (in & $4.4458(1.7503)$ \\
\hline ( $\mathrm{cm}$ (in & $4.4453(1.7501)$ \\
\hline (cm) in & $4.4463(1.7505)$ \\
\hline$(\mathrm{cm}$ (in & $4.4460(1.7504)$ \\
\hline IG $(\mathrm{cm}$ (in & $4.4458(1.750$ \\
\hline
\end{tabular}

Plate Flatness

Plate Finish $(\mu(\mu$ in $))$

Across

$0.30 \quad(11.81)$

With

$0.25 \quad(9.84)$

Orifice Edge

Plate Thickness (cm(in )) $0.315 \quad(0.124)$

$0.315 \quad(0.124)$

Outside Diameter $( \mathrm { cm } ( \text { in } ) ) \longdiv { 1 4 . 9 0 2 ( 5 . 8 6 7 ) }$

Concentricity of hole to oD (cm(in ) $\pm 0.005(0.002)$

Comments

PLATE NUMBER FE $3 / 4 \quad 5 \mathrm{~A}$

Edge Thickness, "e" (cm(in)) 0.0673(0.0265)

Plate Flatness $\mathrm{OK}$

Plate Finish $(\mu(\mu$ in $))$

$\begin{array}{lll}\text { Across } & 0.35 & (13.78) \\ \text { With } & 0.25 & (9.84)\end{array}$

Orifice Edge nick $45^{\circ}$

Plate Thickness (cm(in )) $0.3188(0.1255)$

Outside Diameter (cm(in ) 14.9301 (5.8780)

Concentricity of hole to OD ( cm(in ) $\pm 0.005(0.002)$

Comments

\begin{tabular}{|c|c|c|}
\hline V & (cm) in & )$) 5 .($ \\
\hline LV & ( $\mathrm{cm}$ ( in & $5.0818(2.0007)$ \\
\hline RV & $(\mathrm{cm}$ (in & $5.0810(2.0004)$ \\
\hline $\mathrm{H}$ & in & 2.0 \\
\hline $\mathrm{AVG}$ & $n$ & 8156 \\
\hline
\end{tabular}

PLATE NUMBER FE $3 / 4 \quad 6 \mathrm{~A}$

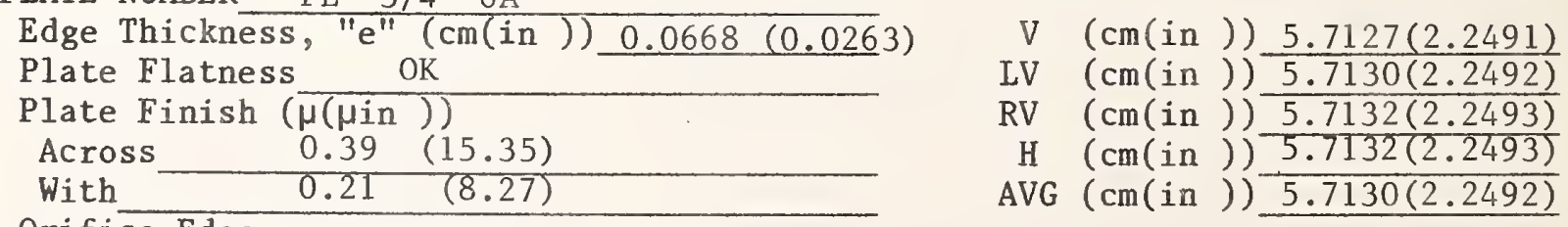

Orifice Edge OK

Plate Thickness (cm(in ) $0.3150 \quad(0.1240)$

Outside Diameter ( $\mathrm{cm}$ (in ) $14.9187 \quad(5.8735)$

Concentricity of hole to OD ( $\mathrm{cm}($ in $)) \pm 0.005(0.002)$

Comments 


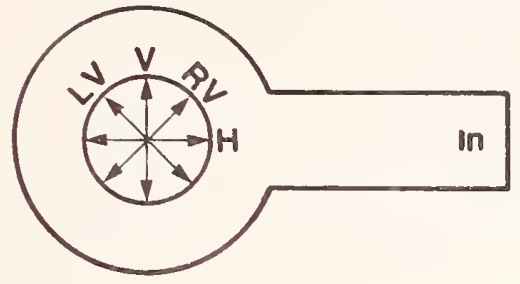

PLATE NUMBER FE $3 / 4 \quad 1 B$

Edge Thickness, "e" (cm(in )) $0.0597(0.0235)$

Plate Flatness $\mathrm{OK}$

Plate Finish $(\mu(\mu i n))$

Across $\quad 0.5 \quad(19.7)$

With $0.25(9.8)$

Orifice Edge nick ( $90^{\circ}$

Plate Thickness ( $\mathrm{cm}$ (in))

Outside Diameter ( $\mathrm{cm}$ (in) ) $14.9225(5.8750)$

Concentricity of hole to $\mathrm{OD}(\mathrm{cm}$ (in ) ) $\pm 0.010 \quad(0.004)$

Comments

PLATE NUMBER

Edge Thickness, "e" (cm(in )) $0.0686(0.027)$

Plate Flatness $\mathrm{OK}$

Plate Finish ( $\mu(\mu$ in $))$

Across $0.35 \quad(13.8)$

With $0.25 \quad(9.8)$

$\mathrm{V}(\mathrm{cm}(\mathrm{in})) 1.5870(0.6248)$

$\mathrm{LV} \quad\left(\mathrm{cm}\right.$ (in ) $\frac{1.5875(0.6250)}{1.5872(0.6249)}$

RV (cm(in ) ) $\frac{1.5872(0.6249)}{1.5872(0.6246)}$

$\mathrm{H} \quad\left(\mathrm{cm}\right.$ (in ) $\frac{1.5865(0.6246)}{1.5865(0.6248)}$

AVG ( cm(in ) $1.5870(0.6248)$

Orifice Edge OK

Plate Thickness (cm(in)) $0.3183(0.1253)$

Outside Diameter (cm(in ) $14.9174(5.8730)$

Concentricity of hole to OD ( cm(in ) $\pm 0.005(0.002)$

Comments

PLATE NUMBER

FE $3 / 4 \quad 3 B$

Edge Thickness, "e" (cm(in )) 0.0724 (0.0285)

Plate Flatness OK

Plate Finish $(\overline{\mu(\mu i n)})$

Across

$0.35(13.78)$

With

$0.20 \quad(7.87)$

Orifice Edge nick $315^{\circ}$

Plate Thickness (cm(in))

$\mathrm{V}$ ( $\mathrm{cm}($ in $)) 2.8578(1.1251)$

LV $\left(\mathrm{cm}\right.$ (in ) $\frac{2.8578(1.1251)}{2.8570(1.1248)}$

RV ( $\mathrm{cm}$ (in ) $\frac{2.8570(1.1248)}{2.8567(1.1247)}$

$\mathrm{H} \quad(\mathrm{cm}($ in $)) \frac{2.8570(1.1248)}{2.8567(1.1247)}$

AVG ( $\mathrm{cm}$ (in ) $2.8572(1.1249)$

Outside Diameter ( $\mathrm{cm}$ (in ) ) 14.0177 (5.8731)

Concentricity of hole to OD (cm(in )) $\pm 0.005 \quad(0.002)$

Comments 


\section{ORIFICE PLATE MEASUREMENTS}

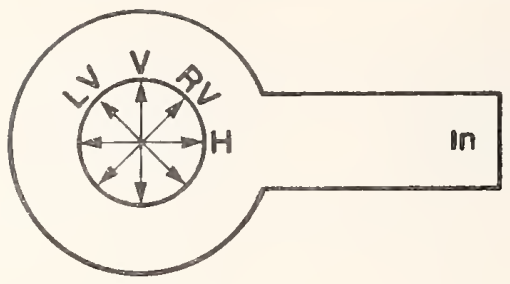

\begin{tabular}{|c|c|c|c|}
\hline FE $\quad 3 / 4 \quad 4 B$ & & & \\
\hline Edge Thickness, "e" (cm(in )) $0.074(0.029)$ & V & $(\mathrm{cm}(\mathrm{in}))$ & $4.4445(1.7498)$ \\
\hline Plate Flatness $\mathrm{H}=0 . \mathrm{K} . \quad \mathrm{V}=0.0038 \mathrm{~cm}$ & $\mathrm{LV}$ & $(\mathrm{cm}($ in $))$ & $4.4450(1.7500)$ \\
\hline Plate Finish $(\mu(\mu i n))$ & RV & $(\mathrm{cm}(\mathrm{in}))$ & $4.4450(1.7500)$ \\
\hline $0.35 \quad(13.78)$ & $\mathrm{H}$ & $(\mathrm{cm}($ in $))$ & $4.4437(1.7495)$ \\
\hline $0.225 \quad(8.86)$ & AVG & $(\mathrm{cm}($ in $))$ & $4.4445(1.7628)$ \\
\hline
\end{tabular}

Plate Thickness (cm(in)) $0.3175(0.1250)$

Outside Diameter ( $\mathrm{cm}($ in $)) 14.920$ (5.874)

Concentricity of hole to $\mathrm{OD}(\mathrm{cm}(\mathrm{in})) \quad 0.005 \quad(0.002)$

Comments

PLATE NUMBER FE $3 / 4 \quad 5 B$

Edge Thickness, "e" (cm(in )) $0.0716(0.0282)$

Plate Flatness

Plate Finish $(\mu(\mu$ in $))$

Across $\quad 0.30 \quad(11.81)$

With $0.17 \quad(6.69)$

Orifice Edge OK

Plate Thickness (cm(in ) ) $0.3162(0.1245)$

Outside Diameter ( $\mathrm{cm}$ (in ) $\longdiv { 1 4 . 9 1 4 9 ( 5 . 8 7 2 0 ) }$

Concentricity of hole to OD ( $\mathrm{cm}($ in $)) \pm 0.005 \quad(0.002)$

Comments

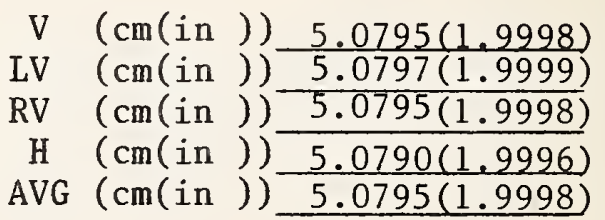

$\mathrm{V} \quad(\mathrm{cm}(\mathrm{in})) \quad 5.0795(1.9998)$

RV (cm(in ) ) $5.0795(1.9998)$

AVG (cm(in ) ) $\frac{5.0790(1.9996)}{5.0795(1.9998)}$

PLATE NUMBER

Edge Thickness, "e" (cm(in )) $0.0714(0.0281)$

Plate Flatness $\mathrm{OK}$

Plate Finish $(\mu(\mu i n))$

Across 0.38

With

$0.21 \quad(8.27)$

Orifice Edge OK

Plate Thickness (cm(in) $0.3099(0.1220)$

Outside Diameter (cm(in) $\longdiv { 1 4 . 9 1 6 \quad ( 5 . 8 7 2 5 ) }$

Concentricity of hole to OD ( $\mathrm{cm}($ in $)) \pm 0.008(0.003)$

Comments

$\begin{aligned} V & (\mathrm{~cm}(\text { in })) \frac{5.7142(2.2497)}{5.7142(2.2497)} \\ \text { LV } & (\mathrm{cm}(\text { in })) \frac{5.7137(2.2495)}{5.7137(2.2495)} \\ \operatorname{RV} & (\mathrm{cm}(\text { in })) \frac{5.7137(2496)}{5.7140(2.24)}\end{aligned}$




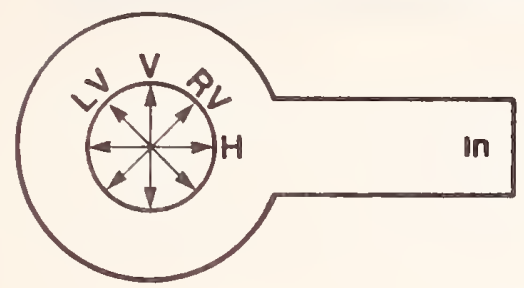

PLATE NUMBER

FE $\quad 5 / 6 \quad 1 \mathrm{~A}$

Edge Thickness, "e" (cm(in )) $0.1333(0.0525)$

Plate Flatness $\mathrm{OK}$

Plate Finish $(\mu(\mu$ in $))$

Across 0.45

$(17.72)$

With $0.35 \quad(13.78)$

Orifice Edge $\mathrm{OK}$

Plate Thickness (cm(in)) $0.310 \quad(0.122)$

Outside Diameter ( $\mathrm{cm}$ (in ) $18.090(7.122)$

Concentricity of hole to OD ( $\overline{c m(\text { in })) \quad \pm 0.020(0.008) ~}$

Comments

PLATE NUMBER FE $\quad 5 / 6 \quad 2 \mathrm{~A}$

Edge Thickness, "e" (cm(in ) $0.1207(0.0475)$

$\mathrm{Plate}$ Flatness

Plate Finish ( $\mu(\mu$ in $))$ Across

With 0.35 $\mathrm{OK}$

Orifice Edge

$0.15 \quad(5.91)$

Plate Thickness (cm(in )) 0.3084(0.1214)

Outside Diameter ( $\mathrm{cm}$ (in ) ) 18.0922(7.1229)

Concentricity of hole to OD (cm(in)) $\pm 0.018(0.007)$

Comments

PLATE NUMBER FE $5 / 6 \quad 3 \mathrm{~A}$

Edge Thickness, "e" (cm(in ) $0.1453(0.0572)$

Plate Flatness $\mathrm{OK}$

Plate Finish $(\mu(\mu$ in $))$ Across

With

Orifice Edge 0.30 $0.20 \quad(7.87)$

$\frac{\mathrm{OK}}{20}$

Plate Thickness (cm(in )) $0.307 \quad(0.121)$

Outside Diameter ( $\mathrm{cm}$ (in ) 18.087 (7.121)

Concentricity of hole to o $\overline{\mathrm{D}(\mathrm{cm}(\mathrm{in})) \pm 0.023(0.009)}$

Comments

\begin{tabular}{|c|c|c|}
\hline V & $(\mathrm{cm}($ in $))$ & $3.8092(1.4997)$ \\
\hline LV & $(\mathrm{cm}($ in $))$ & $30(1.4992$ \\
\hline RV & $(\mathrm{cm}($ in $))$ & $3.8082(1.499$ \\
\hline $\mathrm{H}$ & $(\mathrm{cm}($ in $))$ & $3.8070(1.4$ \\
\hline$A V G$ & $(\mathrm{~cm}$ (in & $2(1.4$ \\
\hline
\end{tabular}

$V(\mathrm{~cm}($ in $)) 2.2225(0.8750)$

LV (cm(in )) $2.2225(0.8750)$

RV ( $\mathrm{cm}$ (in )) $2.2222(0.8749)$

$\mathrm{H}$ ( $\mathrm{cm}$ (in ) $2.2228(0.8751)$

AVG (cm(in )) $2.2225(0.8750)$ 


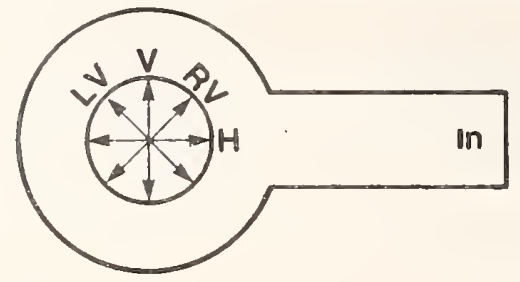

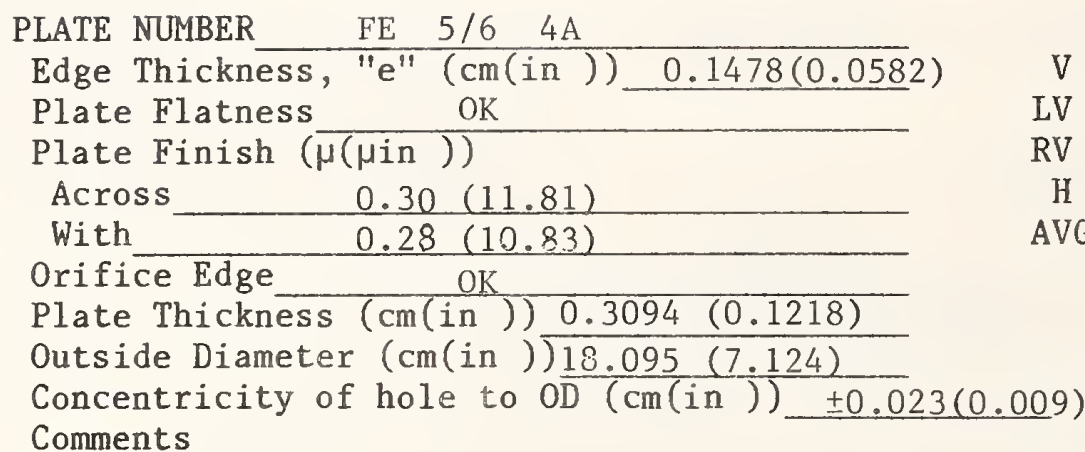

PLATE NUMBER FE $5 / 6 \quad 5 \mathrm{~A}$

Edge Thickness, "e" (cm(in )) $0.1382(0.0544)$

Plate Flatness $\mathrm{OK}$

Plate Finish $(\mu(\mu$ in $))$

Across

With

Orifice Edge $0.30(11.81)$ $0.23(8.86)$

Plate Thickness (cm(in))

Outside Diameter ( $\mathrm{cm}$ (in) $18.087(7.121$ )

Concentricity of hole to oD (cm(in)) $\pm 0.003(0.001)$

Comments
$\mathrm{V} \quad(\mathrm{cm}(\mathrm{in})) 5.7127(2.2491)$

LV (cm(in )) $5.7120(2.2488)$

RV (cm(in ) $5.7142(2.2497)$

$\mathrm{H} \quad(\mathrm{cm}$ (in ) $5.7120(2.2488)$

AVG (cm(in ) $5.7127(2.2491)$

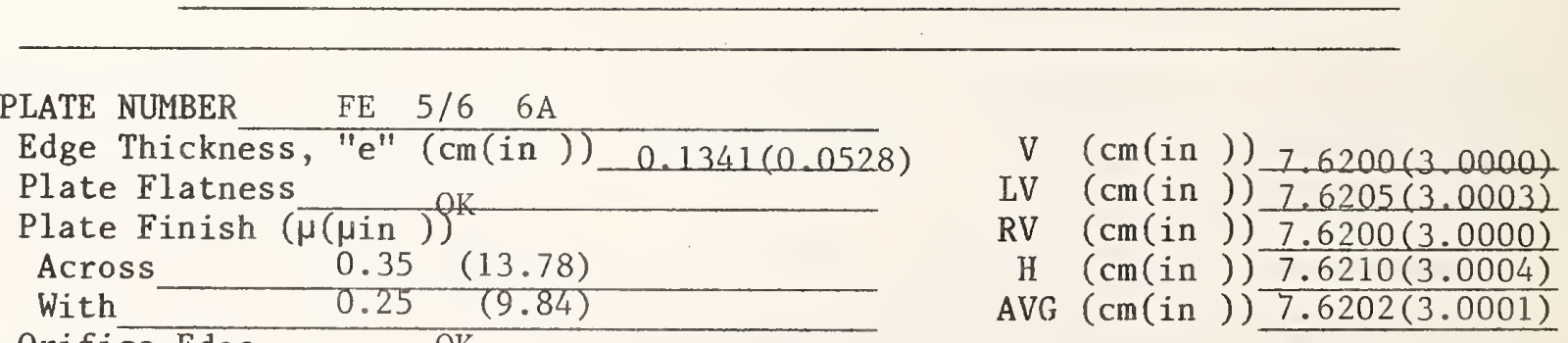

Orifice Edge

Plate Thickness (cm(in )) 0.3061(0.1205)

Outside Diameter (cm(in ) $18.092(7.123)$

Concentricity of hole to $\mathrm{OD}(\mathrm{cm}($ in $)) \pm 0.023(0.009)$

Comments 


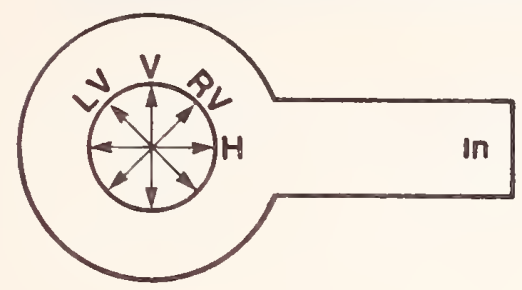

PLATE NUMBER

Edge Thickness, "e" $\mathrm{e}^{\mathrm{f}(\mathrm{cm}(\mathrm{in})) \quad 0.132(0.052)}$

Plate Flatness

Plate Finish ( $\mu(\mu i n))$

Across

$0.53(20.87)$

With

$0.44(17.32)$

Orifice Edge GOOD

Plate Thickness (cm(in )) $0.305(0.120)$

Outside Diameter (cm(in ) $18.0914(7.1226)$

Concentricity of hole to OD ( $\mathrm{cm}($ in $))$

Comments

PLATE NUMBER

Edge Thickness, "e" (cm(in )) $0.127(0.050)$

Plate Flatness

Plate Finish $(\mu(\mu$ in $))$

Across $0.30(11.81)$

With $0.25 \quad(9.84)$

Orifice Edge nicks $0^{\circ} ; 180^{\circ}$

Plate Thickness (cm(in ) $0.305(0.120)$

Outside Diameter ( $\mathrm{cm}$ (in ) $\longdiv { 1 8 . 0 9 5 2 ( 7 . 1 2 4 1 ) }$

Concentricity of hole to OD ( $\mathrm{cm}($ in $))$

Comments
$\mathrm{V} \quad(\mathrm{cm}($ in $)) \quad 2.2230(0.8752)$

LV ( $\mathrm{cm}$ (in ) $\frac{2.2230(0.8752)}{2.03752)}$

RV ( $\mathrm{cm}$ (in )) $2.2230(0.8752)$

$\mathrm{H}(\mathrm{cm}$ (in ) $2.2230(0.8752)$

AVG (cm (in ) $2.2230(0.8752)$

\footnotetext{
PLATE NUMBER FE $5 / 6 \quad 3 B$

Edge Thickness, "e" (cm(in) $\quad 0.145(0.057)$

Plate Flatness $\mathrm{OK}$

Plate Finish $(\overline{\mu(\mu i n)})$

Across

$0.29(11.42)$

With

$0.10 \quad(3.94)$

Orifice Edge GOOD

Plate Thickness (cm(in)) $0.307 \quad(0.121)$

Outside Diameter ( $\mathrm{cm}$ (in )) 18.0889(7.1216)

Concentricity of hole to $\mathrm{OD}(\mathrm{cm}($ in $))$

Comments
} 


\section{ORIFICE PLATE MEASUREMENTS}

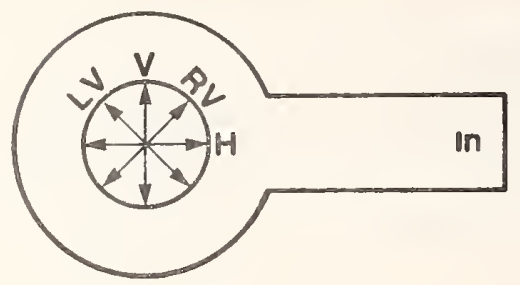

PLATE NUMBER FE $5 / 6 \quad 4 \mathrm{~B}$

Edge Thickness, "e" (cm(in )) $0.152(0.060)$

Plate Flatness $\mathrm{OK}$

Plate Finish ( $\mu(\mu i n))$

Across $0.29(11.42)$

With $0.28(11.02)$

Orifice Edge GOOD

Plate Thickness (cm(in )) $0.312(0.123)$

Outside Diameter ( $\mathrm { cm } ( \text { in } ) \longdiv { 1 8 . 0 9 3 9 ( 7 . 1 2 3 6 ) }$

Concentricity of hole to OD ( $\mathrm{cm}(\mathrm{in}))$

Comments

\begin{tabular}{|c|c|c|}
\hline y & ( $\mathrm{cm}$ (in & $5.7137(2$ \\
\hline $\mathrm{LV}$ & $(\mathrm{cm}$ (in & 5.7 \\
\hline RV & $(\mathrm{cm}($ in $))$ & $5.7142(2.2497$ \\
\hline H & $(\mathrm{cm}($ in $))$ & $5.7137(2.2495$ \\
\hline & (cm) in & 5.71376 \\
\hline
\end{tabular}

PLATE NUMBER

FE $5 / 6 \quad 5 B$

Edge Thickness, "e" (cm(in) $\quad 0.150 \quad(0.059)$

Plate Flatness $\mathrm{V}=0.015 ; \mathrm{H}=\mathrm{OK}$

Plate Finish $(\mu(\mu$ in $))$

Across $\quad 0.28(11.02)$

With

$0.19 \quad(7.48)$

Orifice Edge GOOD

$\mathrm{V} \quad(\mathrm{cm}(\mathrm{in})) \quad 6.6675(2.6250)$

IV $(\mathrm{cm}($ in $)) \frac{6.6665(2.6246)}{6.6667(2.6247)}$

RV (cm(in )) $6.6667(2.6247)$

$\mathrm{H}$ (cm(in )) $6.6675(2.6250)$

AVG (cm(in ) $6.6670(2.6248)$

Plate Thickness (cm(in ) ) 0.305 (0.120)

Outside Diameter ( $\mathrm{cm}($ in $)) \frac{18.0919(7.1228)}{10}$

Concentricity of hole to OD (cm(in))

Comments Surface nick (2320 about 11/16" out

PLATE NUMBER

Edge Thickness, "e" (cm(in ) $\quad 0.135(0.053)$

Plate Flatness

Plate Finish $(\mu(\mu i n))$

\section{Across} $0.25(9.84)$

With

Orifice Edge $0.19(7.48)$

Plate Thickness (cm(in))

Outside Diametex (cm(in ) $\longdiv { 1 8 . 0 9 2 2 ( 7 . 1 2 2 9 ) }$

Concentricity of hole to OD (cm(in))

Comments nicks and burr backside @ outside edge of bevel
$\mathrm{V} \quad(\mathrm{cm}(\mathrm{in})) 7.6180(2.9992)$

LV $(\mathrm{cm}($ in $)) \frac{7.6185(2.9994)}{7.6185(2.9992)}$

RV $\quad(\mathrm{cm}($ in $)) 7.6180(2.9992)$

$\mathrm{H}$ (cm(in ) ) $7.6185(2.9994)$

AVG (cm(in )) $7.6182(2.9993)$ 


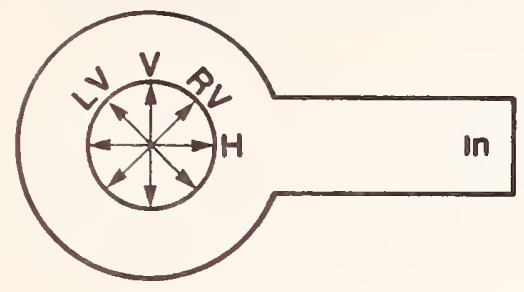

\begin{tabular}{|c|c|c|}
\hline \multirow{3}{*}{ 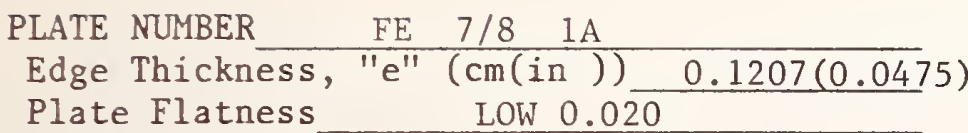 } & & \\
\hline & V & $(\mathrm{cm}($ in $)) 3.1717(1.2487)$ \\
\hline & LV & $(\mathrm{cm}($ in $)) 3.1732(1.2493)$ \\
\hline Plate Finish $(\mu(\mu i n))$ & RV & $(\mathrm{cm}($ in $)) 3.1725(1.2490)$ \\
\hline Across $\quad 0.45(17.72)$ & $\mathrm{H}$ & $(\mathrm{cm}($ in $)) 3.1725(1.2490)$ \\
\hline \multirow[t]{2}{*}{ With $0.40(15.75)$} & AVG & $(\mathrm{cm}($ in $)) 3.1725(1.2490)$ \\
\hline & & \\
\hline \multicolumn{3}{|l|}{ Plate Thickness (cm(in ) $0.297 \quad(0.117)$} \\
\hline \multicolumn{3}{|l|}{ Outside Diameter ( $\mathrm{cm}$ (in ) $25.065(9.868)$} \\
\hline \multicolumn{3}{|c|}{ Concentricity of hole to oD ( $\mathrm{cm}($ in $)) \pm 0.025(0.010)$} \\
\hline \multicolumn{3}{|l|}{ Comments Concave by 0.020} \\
\hline
\end{tabular}
PLATE NUMBER FE $7 / 8 \quad 2 \mathrm{~A}$
Edge Thickness, "e" (cm(in ) $\quad 0.142(0.056)$
Plate Flatness
$\mathrm{V} \quad(\mathrm{cm}$ (in ) $5.7140(2.2496)$
Plate Finish $(\mu(\mu i n))$
Across
$0.43 \quad(16.73)$
With

$0.30 \quad(11.81)$
LV ( $\mathrm{cm}$ (in )) $5.7150(2.2500)$
RV (cm(in )) $5.7153(2.2501)$
$\mathrm{H}(\mathrm{cm}($ in $)) \frac{5.7153(2.2498)}{5.7145(2.2501)}$
AVG (cm(in ) ) $5.7147(2.2499)$

Orifice Edge

OK

Plate Thickness (cm(in )) $0.297(0.117)$

Outside Diameter ( $\mathrm{cm}$ (in ) $25.067(9.869)$

Concentricity of hole to $\mathrm{OD}(\mathrm{cm}($ in $)) \pm 0.028(0.011)$

Comments

PLATE NUMBER $\mathrm{FE} \quad 7 / 8 \quad 3 \mathrm{~A}$

Edge Thickness, "e" (cm(in ) $\quad 0.137(0.054)$

Plate Flatness

Plate Finish ( $\mu(\mu$ in $))^{0 k}$

Across

$0.45(17.72)$

With

$0.30(11.81)$

Orifice Edge

Plate Thickness (cm(in )) $0.297 \quad(0.117)$

Outside Diameter ( $\mathrm{cm}$ (in ) 25.067 (9.869)

Concentricity of hole to OD ( $\mathrm{cm}($ in $)) \pm 0.028(0.011)$

Comments

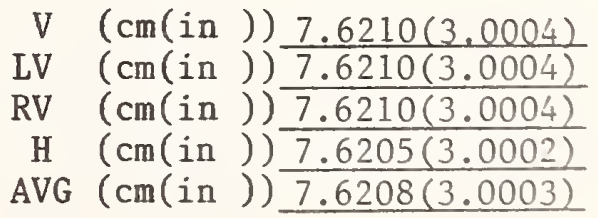




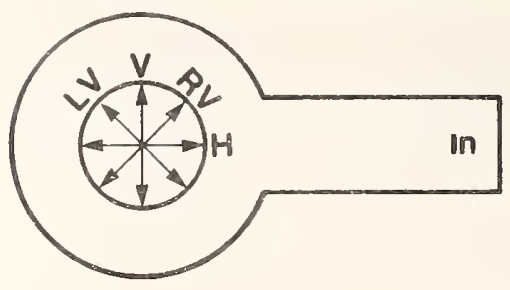

PLATE NUMBER FE $7 / 8 \quad 4 \mathrm{~A}$

Edge Thickness, "e" (cm(in )) $0.130(0.051)$

Plate Flatness $\mathrm{OK}$

Plate Finish ( $\mu(\mu i n))$

Across $0.55(21.65)$

With $0.40(15.75)$

Orifice Edge OK

Plate Thickness (cm(in )) $0.300(0.118)$

Outside Diameter ( $\mathrm{cm}$ (in ) $25.067(9.869)$

Concentricity of hole to $\mathrm{OD}(\mathrm{cm}($ in $)) \pm 0.025(0.010)$

Comments

small nick $@ 90^{\circ}$ and various places on both sides

PLATE NUMBER FE $7 / 8 \quad 5 \mathrm{~A}$

Edge Thickness, "e" (cm(in )) $0.122(0.048)$

Plate Flatness $\quad O K$

Plate Finish $(\overline{\mu(\mu i n}))$

Across $\quad 0.425(16.73)$

With $0.325(12.80)$

Orifice Edge $\quad$ OK

Plate Thickness (cm(in )) $0.292(0.115)$

Outside Diameter ( $\mathrm { cm } ( \text { in } ) \longdiv { 2 5 . 0 6 7 ( 9 . 8 6 9 ) }$

Concentricity of hole to OD ( $\mathrm{cm}($ in $)) \pm 0.028(0.011)$

Comments

$$
\begin{aligned}
& \mathrm{V} \quad(\mathrm{cm}(\text { in })) \quad 10.1597(3.9999) \\
& \text { LV (cm(in )) } 10.1595(3.9998) \\
& \text { RV (cm(in )) 10.1605(4.0002) } \\
& \text { H ( cm(in )) } 10.1595(3.9998) \\
& \text { AVG (cm(in )) } 10.1597 \text { (3.9999) }
\end{aligned}
$$

$\mathrm{V} \quad(\mathrm{cm}($ in $)) 8.8885(3.4994)$

RV (cm(in) $8.8892(3.4997)$

$\mathrm{H} \quad(\mathrm{cm}($ in $)) \frac{8.8885(3.4994)}{8.8885(3.4994)}$

AVG (cm(in ) $8.8885(3.4994)$

\section{Comments}

PLATE NUMBER EE 7/8 6A

Edge Thickness, "e" (cm(in )) 0.132(0.052)

Plate Flatness

Plate Finish $(\overline{\mu(\mu i n))}$

Across

With

Orifice Edge $0.40(15.75)$

Plate Thickness ( $\mathrm{cm}($ in $))$

Outside Diameter (cm(in)

Outside Diater (cm (in ) $25.067(9.869)$

Concentricity of hole to OD ( $\mathrm{cm}($ in $)) \_ \pm 0.028(0.011)$

Comments

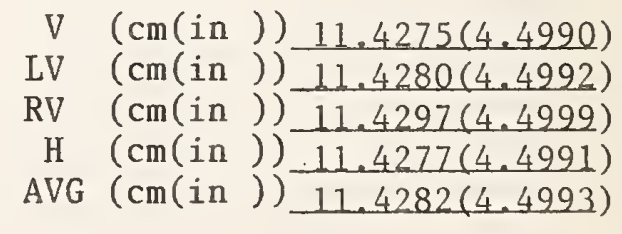




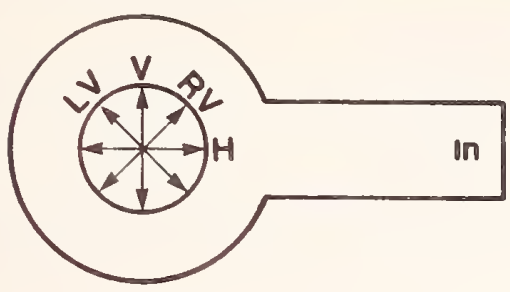

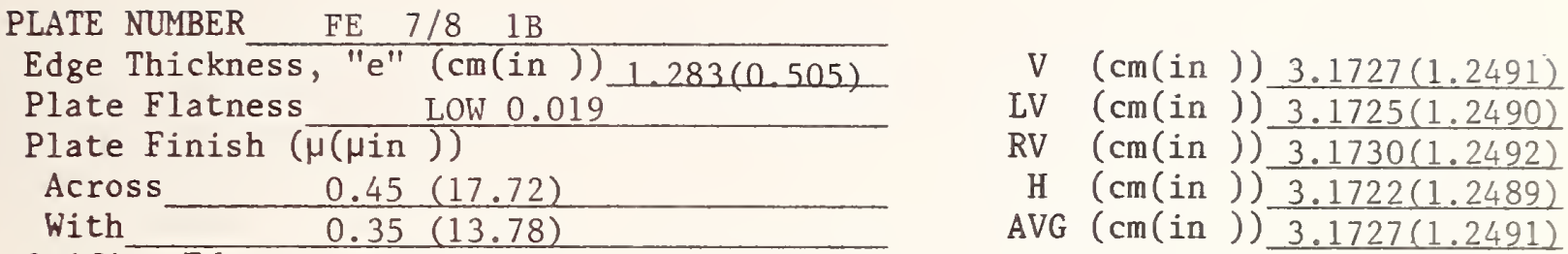

Orifice Edge OK

Plate Thickness (cm(in )) $0.302(0.119)$

Outside Diameter ( $\mathrm{cm}($ in $)) 25.070(9.870)$

Concentricity of hole to OD ( $\mathrm{cm}($ in $)) \pm 0.030(0.012)$

Comments

Concave by 0.020 in. nicks on both sides

PLATE NUMBER FE $7 / 8$ 2B

\begin{tabular}{|c|c|c|}
\hline Edge Thickness, "e" (cm(in )) $0.127(0.050)$ & $\mathrm{V}$ & $(\mathrm{cm}($ in $)) 5.7145(2.2498)$ \\
\hline Plate Flatness $\quad$ OK & $\mathrm{LV}$ & $(\mathrm{cm}($ in $)) 5.71 .58(2.2503)$ \\
\hline Le Finish $(\mu(\mu i n))$ & RV & $(\mathrm{cm}($ in $)) 5.7145(2.2498)$ \\
\hline $0.45(17.72)$ & $\mathrm{H}$ & $5.7153(2.2501)$ \\
\hline $0.40(15.75)$ & AVG & $(\mathrm{cm}($ in $)) .5 .7150(2.250$ \\
\hline
\end{tabular}

Orifice Edge OK

Plate Thickness (cm(in)) $0.297(0.117)$

Outside Diameter ( $\mathrm{cm}$ (in )) 25.065(9.868)

Concentricity of hole to OD ( $\mathrm{cm}($ in $)) \pm 0.028(0.011)$

Comments

\begin{tabular}{|c|c|c|c|}
\hline PLATE NUMBER & & & \\
\hline Edge Thickness, "e" (cm(in )) $0.130(0.051)$ & $V$ & $(\mathrm{~cm}($ in $))$ & $7.6208(3.0003)$ \\
\hline Plate Flatness $\quad$ OK & $\mathrm{LV}$ & $(\mathrm{cm}($ in $))$ & $7.6210(3.0004)$ \\
\hline Plate Finish $(\mu(\mu i n))$ & RV & $(\mathrm{cm}($ in $))$ & $7.6213(3.0005)$ \\
\hline $0.40(15.75)$ & $\mathrm{H}$ & $(\mathrm{cm}($ in $))$ & $7.6210(3.0004)$ \\
\hline $0.25 \quad(9.84)$ & AVG & $(\mathrm{cm}(\text { in }))^{-}$ & $7.6210(3.000 \div)$ \\
\hline
\end{tabular}

Orifice Edge $\frac{\text { OK }}{\text { Plate Thickness (cm(in }))} 0.300(0.118)$
Old

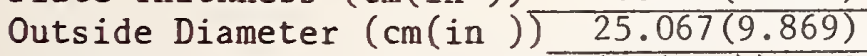

Concentricity of hole to $\mathrm{OD}(\mathrm{cm}($ in $)) \pm 0.028(0.011)$

Comments

nicks at various places on both sides 


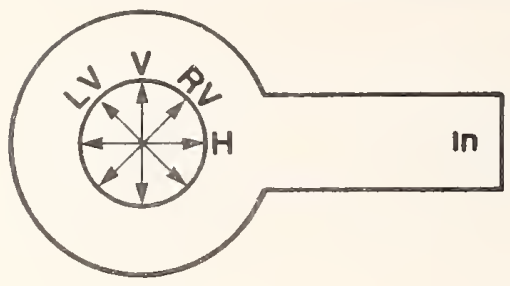

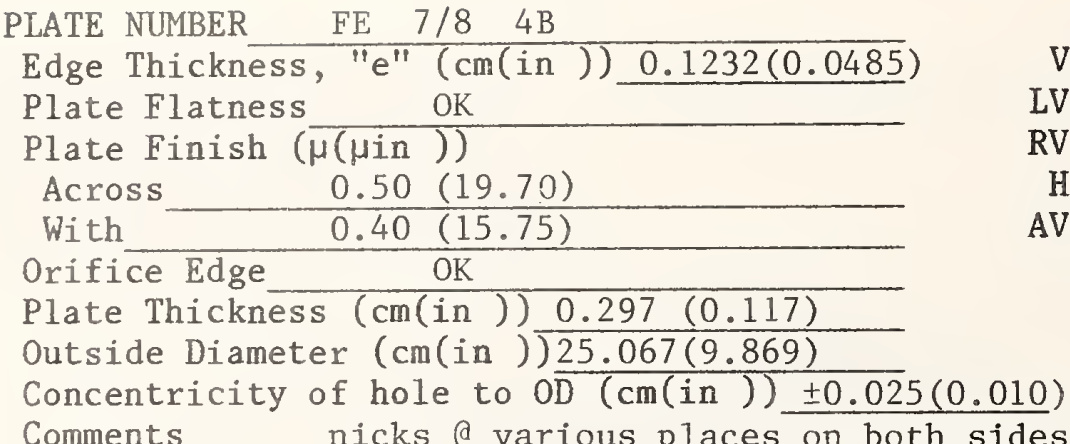

PLATE NUMBER FE $7 / 8$ 5B
Edge Thickness, "e" (cm(in ) $0.1333(0.0525)$

Plate Flatness $\quad$ OK

Plate Finish $(\mu(\mu i n))$

Across $\quad 0.50(19.70)$

With $0.30(11.81)$

Orifice Edge OK

Plate Thickness (cm(in )) $0.2997(0.118)$

Outside Diameter (cm(in ) 25.070 (9.870)

Concentricity of hole to $\mathrm{OD}(\mathrm{cm}($ in $)) \pm 0.030(0.012)$

Comments

PLATE NUMBER FE $7 / 8 \quad 6 \mathrm{~B}$

Edge Thickness, "e" (cm(in )) 0.109(0.043)

Plate Flatness $\quad \mathrm{OK}$
Plate Finish $(\mu(\mu$ in $))$

Across $\quad 0.40(15.75)$

With $0.25(9.84)$

Orifice Edge OK

Plate Thickness (cm(in)) $0.297(0.117)$

Outside Diameter (cm(in )) 25.067(9.869)

Concentricity of hole to oD $(\mathrm{cm}(\mathrm{in})) \pm 0.028 .(0.011)$

Comments

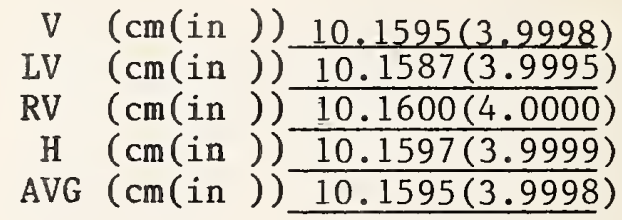

$\mathrm{V} \quad(\mathrm{cm}(\mathrm{in})) \quad 8.8882(3.4993)$

LV $(\mathrm{cm}($ in $)) \frac{8.8880(3.4992)}{8.880}$

$\mathrm{RV} \quad\left(\mathrm{cm}\right.$ (in ) $\frac{8.88882(3.4993)}{8.8882(3.4994)}$

$\mathrm{H} \quad(\mathrm{cm}($ in $)) \frac{8.8885(3.4994)}{8.8885(3.4993)}$

AVG $(\mathrm{cm}($ in $)) \frac{8.8885(3.4994)}{8.8882(3.4993)}$ 


\section{APPENDIX III.}

Nieasurements of $\mathrm{Flow}$ Conditioners

The flow conditioners were measured at NBS-Boulder, Colorado. The hole pattern in the three plates of each conditioner was in general the same and the plates were mounted so that the holes of each plate lined up with the other plates. The hole pattern was in rows with the holes of the alternate rows offset. If a support rod lay in the position so as to impose on a hole, the hole was left out. All plates have an odd number of rows and the center hole of the center row was at the center line of the plate. The measurments for position of the rows and the rods were made from the center of this hole. 
Flow Conditioner Measurements

Flow conditioner nominal size

4 inch

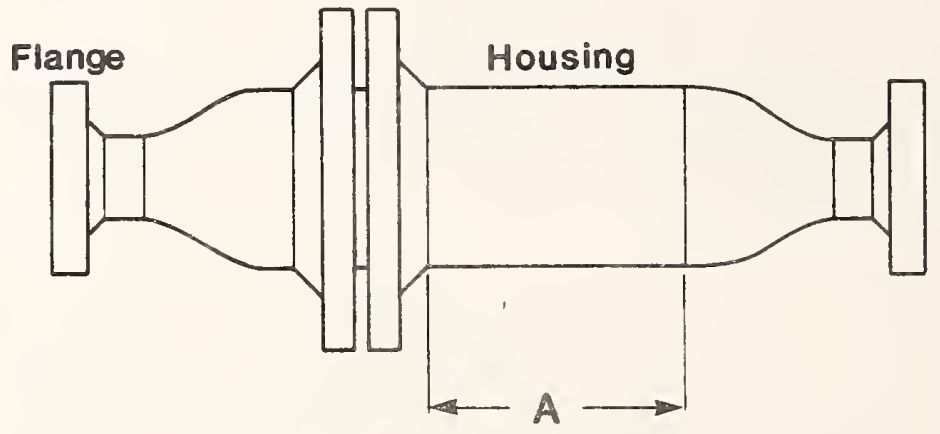

Nominal pipe size section A 4 inch

Inlet and outlet flange $\frac{3 \text { inch }}{3 \text { to } 4 \text { inch }}$

Inlet and outlet reducer 3 to 4 inch

Overall length, cm (in) $82.07(32.31)$

Straight pipe length, A, cm (in) 20.16(7.94)

600 psi ANSI

Schedule 40 
Flow Conditioner Nominal Size 4 inch
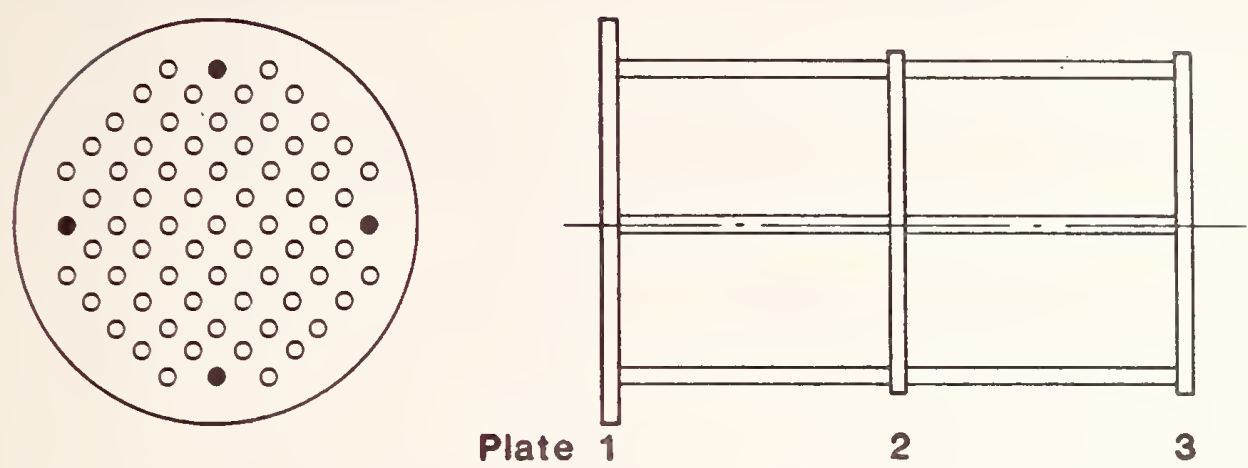

Number of support rods 4 equally spaced

Center line of rod to center line of plates, cm (in) 3.790(1.492)

Rod diameter $1.900(0.748)$

Distance between plate 1 and 2 8.776(3.455)

Distance between plate 2 and 3 8.682(3.418)

Diameter, cm (in)

Thickness, cm (in)

Number of holes

Average hole diameter, cm (in)

Average distance between holes, cm (in)

Upstream chamfer

Chamfer depth, cm (in)

Number of rows

\begin{tabular}{|c|c|c|}
\hline Plate 1 & Plate 2 & Plate 3 \\
\hline 17.457 & 10.160 & 10.160 \\
$(6.873)$ & $(4.000)$ & $(4.000)$ \\
\hline 0.947 & 0.960 & 0.960 \\
$(0.373)$ & $(0.378)$ & $(0.378)$ \\
\hline 53 & 53 & 53 \\
\hline 0.622 & 0.617 & 0.622 \\
$(0.245)$ & $(0.243)$ & $(0.245)$ \\
\hline 0.950 & 0.947 & 0.947 \\
$(0.374)$ & $(0.373)$ & $(0.373)$ \\
\hline $90^{\circ}$ & $90^{\circ}$ & $90^{\circ}$ \\
\hline 0.094 & 0.091 & 0.091 \\
$(0.037)$ & $(0.036)$ & $(0.036)$ \\
\hline 11 & 11 & 11 \\
\hline
\end{tabular}




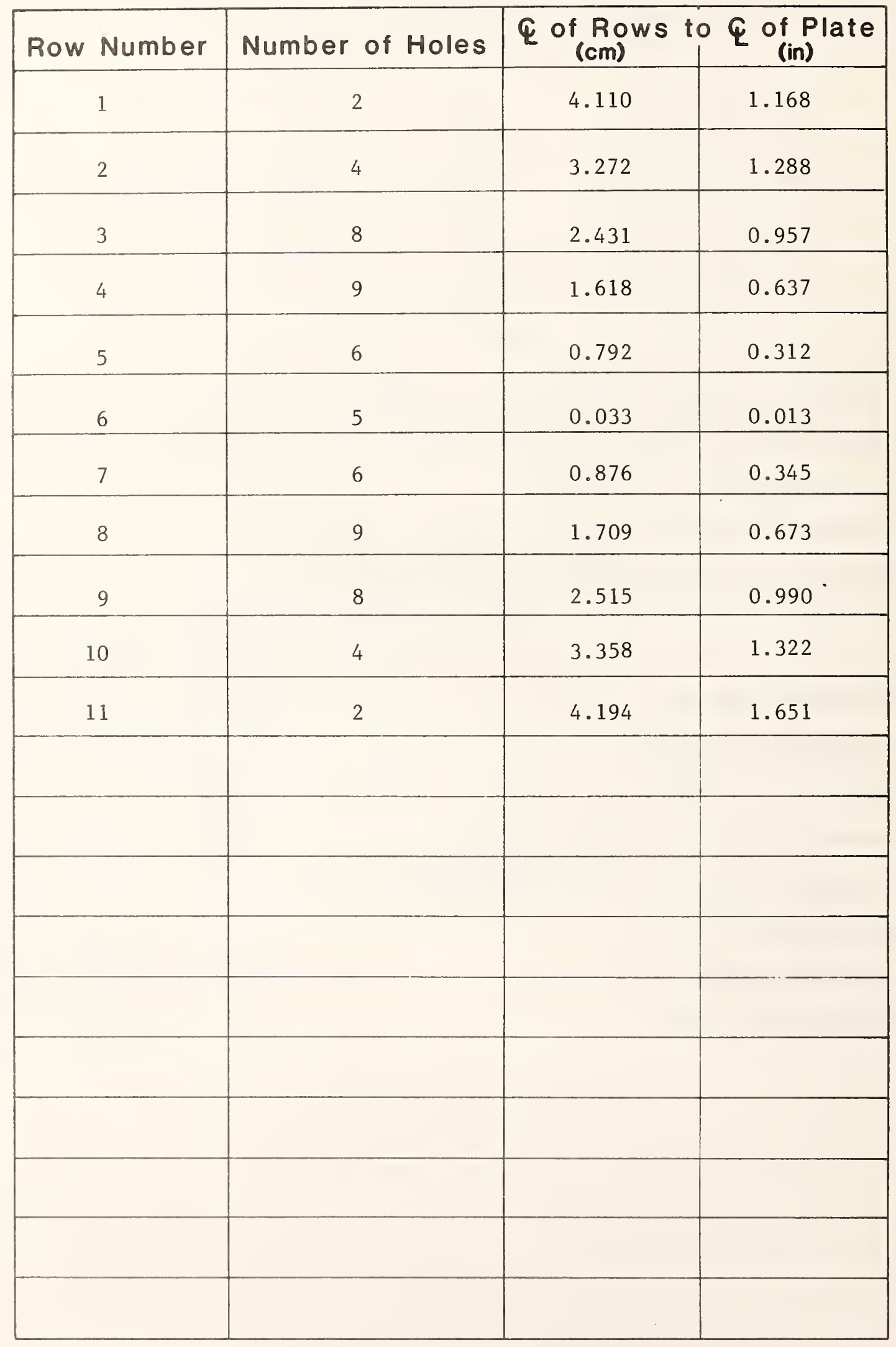


Flow Conditioner Measurements

Flow conditioner nominal size 6 inch

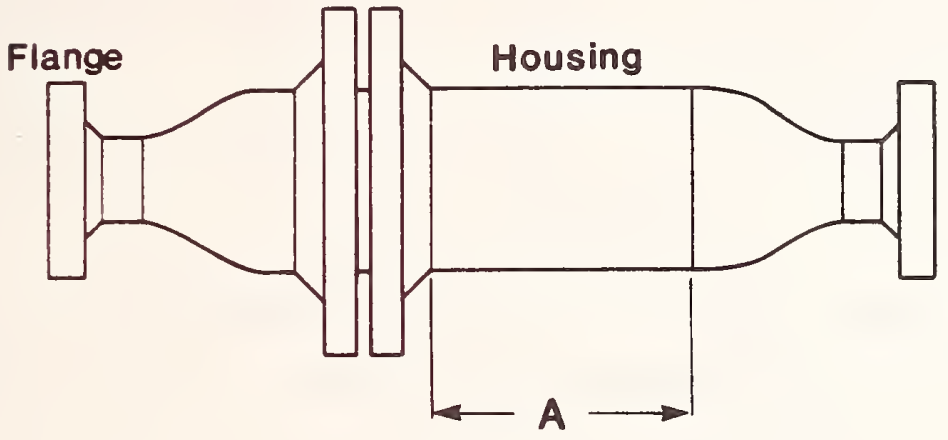

Nominal pipe size section A 6 inch

Inlet and outlet flange 4 inch

Inlet and outlet reducer 4 to 6 inch

Overall length, cm (in) $105.95(41.71)$

Straight pipe length, A, cm (in) 30.48(12.00)

600 psi ANSI

Schedule 40 
Flow Conditioner Nominal Size 6 inch
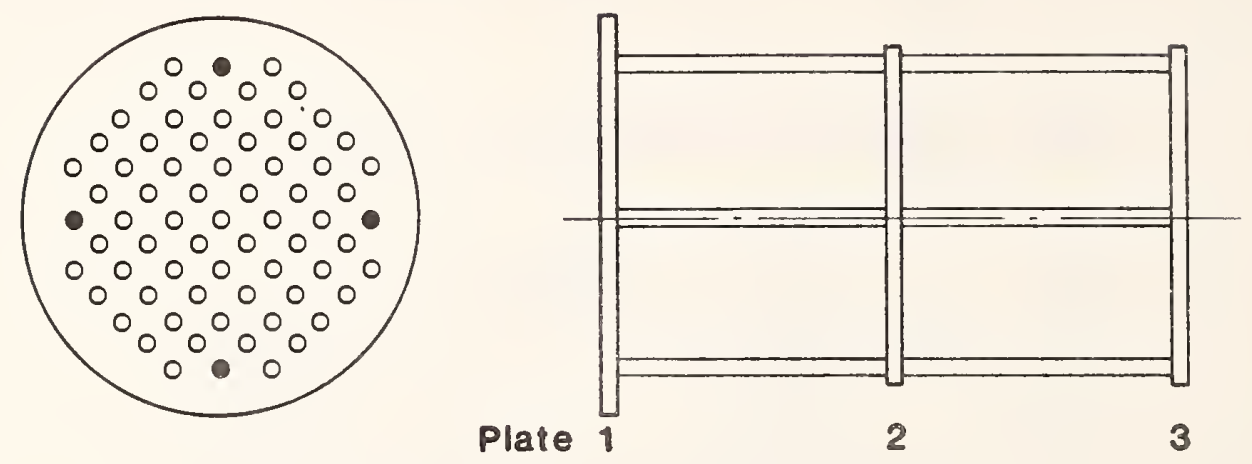

Number of support rods 4 equally spaced

Center line of rod to center line of plates, cm (in) $6.342(2.497)$

Rod diameter $1.905(0.750)$

Distance between plate 1 and 2 14.389(5.665)

Distance between plate 2 and 3 13.378(5.267)

Diameter, cm (in)

Thickness, cm (in)

Number of holes

Average hole diameter, cm (in)

Average distance between holes, cm (in)

Upstream chamfer

Chamfer depth, cm (in)

Number of rows

\begin{tabular}{|c|c|c|}
\hline Plate 1 & Plate 2 & Plate 3 \\
\hline 26.662 & $15.343)$ & 15.3212 \\
$(10.497)$ & $(6.042)$ & 0.032 \\
\hline 1.016 & 0.927 & 0.927 \\
$(0.400)$ & $(0.365)$ & $(0.365)$ \\
\hline 87 & 87 & 87 \\
\hline 1.041 & 1.041 & 1.039 \\
$(0.410)$ & $(0.410)$ & $(0.409)$ \\
\hline$(0.364)$ & $(0.567)$ & $(0.3638)$ \\
\hline $82^{\circ}$ & $82^{\circ}$ & $82^{\circ}$ \\
\hline 0.152 & 0.102 & 0.102 \\
$(0.060)$ & $(0.040)$ & $(0.040)$ \\
\hline 11 & 11 & 11 \\
\hline
\end{tabular}




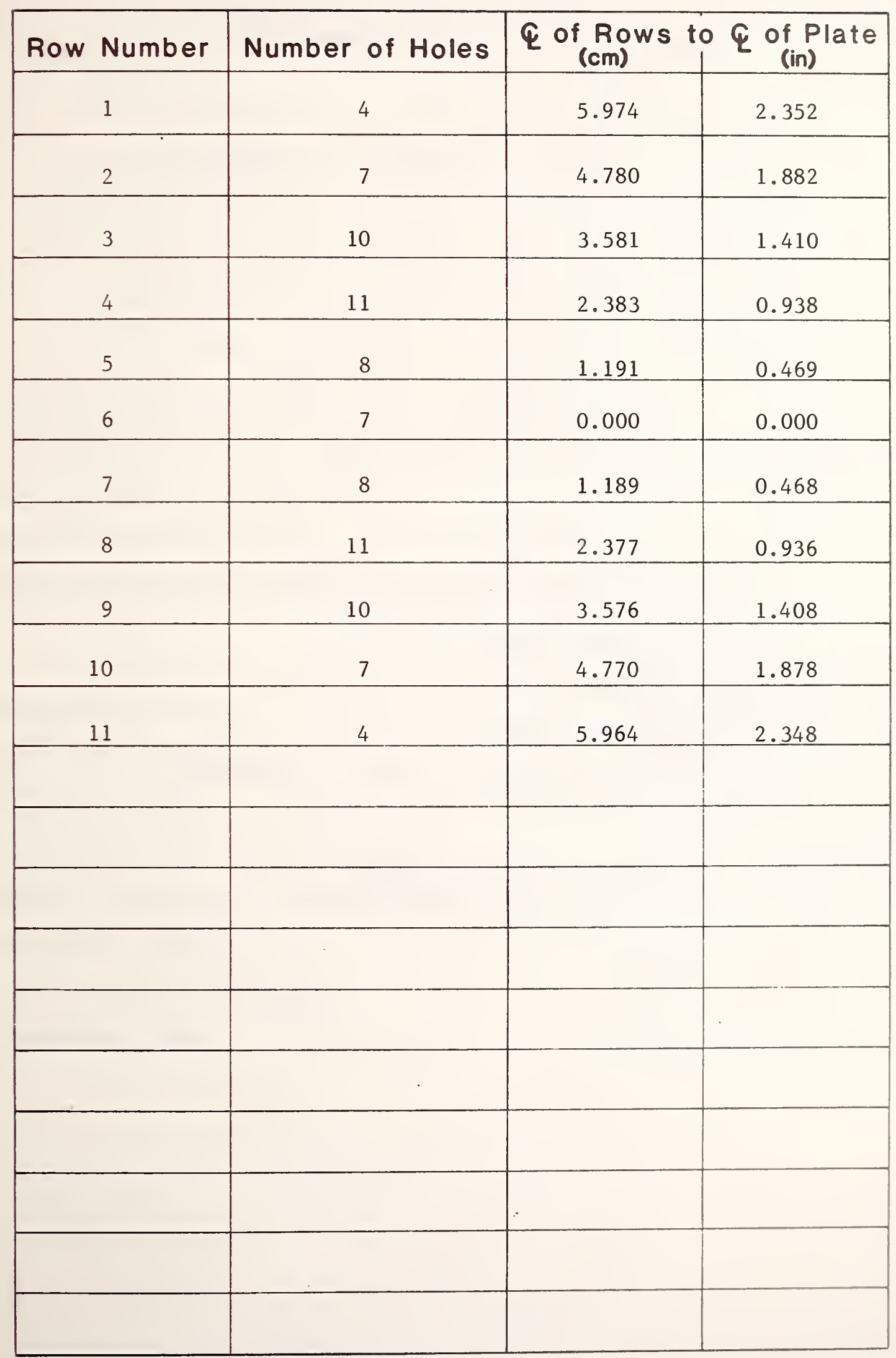


Flow Conditioner Measurements

Flow conditioner nominal size 10 inch

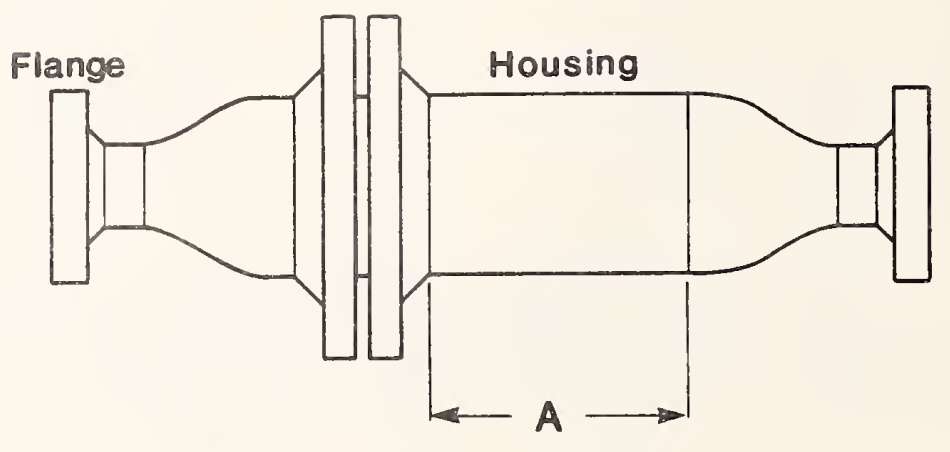

Nominal pipe size section A 10 inch

Inlet and outlet flange 6 inch

Inlet and outlet reducer 6 to 10 inch

Overall length, cm (in) $139.0(54.72)$

Straight pipe length, A, cm (in) 47.47(18.69)

600 psi ANSI

Schedule 40 
Flow Conditioner Nominal Size 10 inch
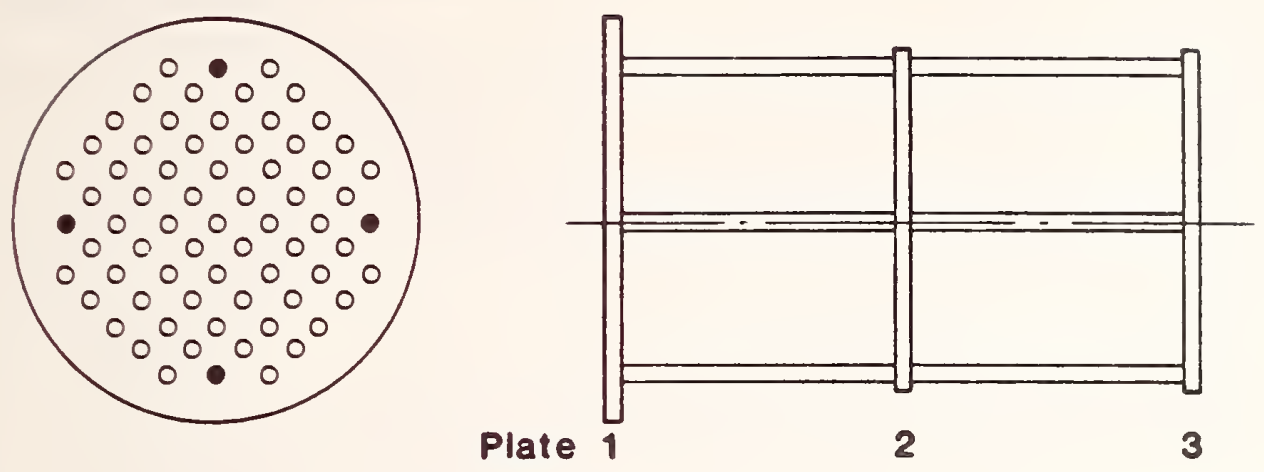

Number of support rods 4 equally spaced

Center line of rod to center line of plates, cm (in) $11.049(4.350)$

Rod diameter $1.895 \quad(0.746)$

Distance between plate 1 and $223.293(9.446)$

Distance between plate 2 and 3 23.998(9.448)

Diameter, cm (in)

Thickness, cm (in)

Number of holes

Average hole diameter, cm (in)

Average distance between holes, cm (in)

Upstream chamfer

Chamfer depth, cm (in)

Number of rows

\begin{tabular}{|c|c|c|}
\hline Plate 1 & Plate 2 & Plate 3 \\
\hline $\begin{array}{c}33.973 \\
13.375)\end{array}$ & $\begin{array}{c}25.403 \\
(10.001)\end{array}$ & $\begin{array}{c}25.403 \\
(10.001)\end{array}$ \\
\hline 0.960 & 0.983 & 0.970 \\
$(0.378)$ & $(0.387)$ & $(0.382)$ \\
\hline 291 & 291 & 291 \\
\hline $\begin{array}{c}1.036 \\
(0.408)\end{array}$ & $\begin{array}{c}1.039 \\
(0.409)\end{array}$ & $\begin{array}{c}1.036 \\
(0.408)\end{array}$ \\
\hline $\begin{array}{c}1.346 \\
(0.530)\end{array}$ & $\begin{array}{c}1.351 \\
(0.532)\end{array}$ & $\begin{array}{c}1.346 \\
(0.530)\end{array}$ \\
\hline $89^{\circ}$ & $89^{\circ}$ & \\
\hline 0.157 & 0.157 & 0.157 \\
$(0.062)$ & $(0.062)$ & $(0.062)$ \\
\hline & & \\
21 & 21 & 21 \\
\hline
\end{tabular}




\begin{tabular}{|c|c|c|c|}
\hline Row Number & Number of Holes & $\mathcal{E}$ of Rows $\mathrm{t}$ & $E$ of Plate \\
\hline 1 & 4 & 11.902 & 4.686 \\
\hline 2 & 8 & 10.716 & 4.219 \\
\hline 3 & 13 & 9.530 & 3.752 \\
\hline 4 & 14 & 8.341 & 3.284 \\
\hline 5 & 15 & 7.135 & 2.809 \\
\hline 6 & 16 & 5.954 & 2.344 \\
\hline 7 & 17 & 4.747 & 1.869 \\
\hline 8 & 18 & 3.576 & 1.408 \\
\hline 9 & 19 & 2.367 & 0.932 \\
\hline 10 & 14 & 1.171 & 0.461 \\
\hline 11 & 15 & 0.020 & 0.008 \\
\hline 12 & 14 & 1.207 & 0.475 \\
\hline 13 & 19 & 2.405 & 0.947 \\
\hline 14 & 18 & 3.579 & 1.409 \\
\hline 15 & 17 & 4.773 & 1.879 \\
\hline 16 & 16 & 5.969 & 2.350 \\
\hline 17 & 15 & 7.150 & 2.815 \\
\hline 18 & 14 & 8.362 & 3.292 \\
\hline 19 & 13 & 9.530 & 3.752 \\
\hline 20 & 8 & 10.721 & 4.221 \\
\hline 21 & 4 & 11.885 & 4.679 \\
\hline
\end{tabular}




\begin{tabular}{|c|c|c|c|}
\hline $\begin{array}{l}\text { U.S. OEPT. OF COMM. } \\
\text { BIBLIOGRAPHIC DATA } \\
\text { SHEET (See instructions) }\end{array}$ & $\begin{array}{l}\text { 1. PUBLICATION OR } \\
\text { REPORT NO. } \\
\text { NBSIR } 83-1685\end{array}$ & 2. Performing Organ. Report No. & $\begin{array}{l}\text { 3. Publication Date } \\
\text { August } 1983\end{array}$ \\
\hline
\end{tabular}

Gas Orifice Meter Discharge Coefficients As Determined by Mass Flow Measurements

5. $A \cup T H O R(S)$

D. B. Mann, J.A. Brennan, C.F. Sindt, J.F. LaBrecque, S. McManus, C. Kneebone

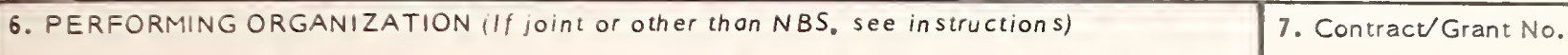

NATIONAL BUREAU OF STANDARDS

DEPARTMENT OF COMMERCE

WASHINGTON, D.C. 20234

9. SPONSORING ORGANIZATION NAME AND COMPLETE ADDRESS (Street, City, State, ZIP)

8. Type of Report \& Period Covered NBSIR

Gas Research Institute

8600 West Bryn Mawr Avenue

Chicago, IL 60631

10. SUPPLEMENTARY NOTES

Document describes a computer program; SF-185, FIPS Software Summary, is attached.

11. ABSTRACT (A 200-word or less factual summary of most significant information. If document includes a significant bibliogrophy or literoture survey, mention it here)

Performance data of gas orifice meter runs and plates have been generated under a U.S. gas industry supported program. The data have been developed using nitrogen gas and a unique NBS gas flow measurement facility capable of directly measuring the mass of gas metered by the orifice device. Direct comparison of predictions from empirical equations can now be made at orifice bore Reynolds Numbers near four million. Two meter runs for each of four nominal line sizes and two sets of orifice plates with up to six beta ratios per set were interchanged in order to develop full meter performance characteristics. Orifice meter and flow reference system data are used to calculate discharge and expansion coefficients which in turn are compared to those derived from existing equations. Orifice meter performance data and system descriptions are provided.

12. KEY WORDS (Six to twelve entries; alphabetical order; capitalize only proper names; and seporate key words by semicolons) discharge coefficients; flow measurement; flowmeter; gas flow; mass flow; orifice meter

13. AVAILABILITY

X] Unlimited

For Official Distribution. Do Not Release to NTIS

Order From Superintendent of Documents, U.S. Government Printing Office, Washington, D.C. 20402.

X Order From National Technical Information Service (NTIS), Springfield, VA. 22161

14. NO. OF

PRINTED PAGES

135

15. Price

$\$ 14.50$ 


